Publ. RIMS, Kyoto Univ.

15 (1979), 871-972

\title{
Holonomic Quantum Fields. IV
}

By

Mikio SATO*, Tetsuji MiWA* and Michio JIMBO*

\section{Introduction}

This is the fourth part in our series of papers on Holonomic Quantum Fields, [1], [2] and [3].

In $I$ we prepared the theory of rotation in orthogonal vector spaces, and in II we exploited it to solve the Riemann-Hilbert problem. In III we developed the deformation theory of multivalued solutions of the 2dimensional Euclidean Klein-Gordon and Dirac equations. The present chapter deals with the operator thoery in 2-dimensional space-time.

In Section 4.1 we construct the holonomic quantum field $\varphi_{B}(a)$ satisfying the following commutation relations with the neutral free Bose field $\phi(x)$ $([4])$.

$$
\text { (4. 0.1) } \quad \varphi_{B}(a) \phi(x)=\left\{\begin{aligned}
\phi(x) \varphi_{B}(a) & \left(x^{+}>a^{+}, x^{-}<a^{-}\right) \\
-\phi(x) \varphi_{B}(a) & \left(x^{+}<a^{+}, x^{-}>a^{-}\right) .
\end{aligned}\right.
$$

Since $\phi(x)$ obeys the Bose statistics we need the theory of rotations in symplectic vector spaces, which is given in the Appendix. The computation is carried out in the case of $n$-dimensional space-time by means of a kind of the Wiener-Hopf method.

In Section 4.2 we construct the operator $\varphi_{F}(a)$ satisfying the following (see [1]).

$$
\text { (4. 0.2) } \quad \varphi_{F}(a) \phi_{ \pm}(x)=\left\{\begin{aligned}
\phi_{ \pm}(x) \varphi_{F}(a) & \left(x^{+}>a^{+}, x^{-}<a^{-}\right) \\
-\psi_{ \pm}(x) \varphi_{F}(a) & \left(x^{+}<a^{+}, x^{-}>a^{-}\right) .
\end{aligned}\right.
$$

Here $\psi(x)={ }^{t}\left(\psi_{+}(x), \psi_{-}(x)\right)$ is the neutral free Fermi field. This time we exploit the results in I.

In Section 4.3 we introduce the complex free Bose fields $\phi(x)$ and $\phi^{*}(x)$.

Received October 20, 1978.

* Research Institute for Mathematical Sciences, Kyoto University.

+ Partially supported by Sakkokai Foundation. 
This enables us to formulate more general commutation relations ([7], [9]), namely for $l \in \boldsymbol{C}-\boldsymbol{Z}$

$$
\text { (4.0.3) } \begin{aligned}
\varphi_{B}(a ; l) \phi(x) & = \begin{cases}\phi(x) \varphi_{B}(a ; l) & \left(x^{+}>a^{+}, x^{-}<a^{-}\right) \\
e^{2 \pi i l} \phi(x) \varphi_{B}(a ; l) & \left(x^{+}<a^{+}, x^{-}>a^{-}\right),\end{cases} \\
\varphi_{B}(a ; l) \phi^{*}(x) & = \begin{cases}\phi^{*}(x) \varphi_{B}(a ; l) & \left(x^{+}>a^{+}, x^{-}<a^{-}\right) \\
e^{-2 \pi i l} \phi^{*}(x) \varphi_{B}(a ; l) & \left(x^{+}<a^{+}, x^{-}>a^{-}\right) .\end{cases}
\end{aligned}
$$

The local operator expansion of the product $\phi(x) \varphi_{B}(a ; l)$ is given. As the coefficients we obtain a series of operators $\varphi_{l^{\prime}}^{B}(a ; l)$. The orthogonal case is similarly treated.

In Section 4.4 we exploit the local operator expansion to study the analytic behavior of vacuum expectation values of products of our operators, and conclude that

$$
\pi \frac{\left\langle\phi^{*}\left(x^{*}\right) \varphi_{B}\left(a_{1} ; l_{1}\right) \cdots \varphi_{B}\left(a_{n} ; l_{n}\right) \phi(x)\right\rangle}{\left\langle\varphi_{B}\left(a_{1} ; l_{1}\right) \cdots \varphi_{B}\left(a_{n} ; l_{n}\right)\right\rangle}
$$

and

$$
2 \sin \pi l_{\nu}\left\langle\frac{\left\langle\varphi_{B}\left(a_{1} ; l_{1}\right) \cdots \varphi_{l_{\nu}}^{B^{*}}\left(a_{\nu} ; l_{\nu}\right) \cdots \varphi_{B}\left(a_{n} ; l_{n}\right) \phi(x)\right\rangle}{\left\langle\varphi_{B}\left(a_{1} ; l_{1}\right) \cdots \varphi_{B}\left(a_{n} ; l_{n}\right)\right\rangle}\right.
$$

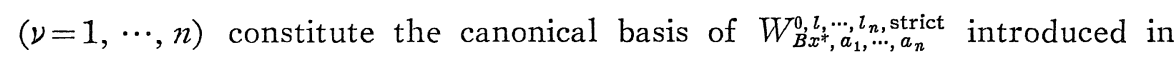
III of [3]. This provides us with a constructive proof on the existence of the above basis, independent of the one given in III. As a by-product the logarithmic derivative of the $n$-point function

$$
d \log \left\langle\varphi_{B}\left(a_{1} ; l_{1}\right) \cdots \varphi_{B}\left(a_{n} ; l_{n}\right)\right\rangle
$$

is shown to coincide with the closed 1 -form $-\omega$ defined in III. Thus the $n$-point functions of our field operators are characterized by solutions of the deformation equations given in [3] and [5]. These results with minor modifications are all valid in the orthogonal case as well (see [5], [7]). In particular we show the following simple relation of [9]

$$
(4.0 .4) \quad\left\langle\varphi_{B}\left(a_{1} ; l_{1}+\frac{1}{2}\right) \cdots \varphi_{B}\left(a_{n} ; l_{n}+\frac{1}{2}\right)\right\rangle\left\langle\varphi_{F}\left(a_{1} ; l_{1}\right) \cdots \varphi_{F}\left(a_{n} ; l_{n}\right)\right\rangle=1 \text {. }
$$

The generalization to the case including the parameter $\Lambda$ (cf. [1], [7]) is similarly treated. This clarifies the origin of the $n(n-1) / 2$ dimensional family of global monodromy introduced in III. 
In Section 4. 5 we exploit the product formula in I or in the Appendix to give a convergence proof of products of our operators. In particular, we give convergent infinite series expressions for vacuum expectation values given in Section 4. 4. Thus the algebraic results in Section 4.4 acquires a rigorous basis. We also prove the micro-causality of our field operators.

In the final $\S 4.6$ we discuss in detail the original case based on neutral free fields. Along with the operators $\varphi_{B}(a)(\S 4.1)$ and $\varphi_{F}(a)$ (§4.2) we shall also deal with the ones $\varphi^{B}(a)={ }^{t}\left(\varphi_{+}^{B}(a), \varphi_{-}^{B}(a)\right)$ and $\varphi^{F}(a)$, which appear as the leading coefficients of the local expansions for $\phi(x) \varphi_{B}(a)$ and $\psi(x) \varphi_{F}(a)$ respectively ([5]). The latter ones constitute physical models of Fermi and Bose fields, satisfying Lorentz covariance, microcausality and asymptotic completeness condition. We note here the remarkable reciprocity between these Fermi and Bose fields ([9]). Namely the field $\varphi_{B}(a)$, constructed on the basis of free Boson $\phi(x)$, represents a strongly interacting Fermi field with asymptotic free Fermi fields $\psi_{ \pm}^{B}(a)$, while the field $\varphi^{F}(a)$, constructed from free Fermion $\psi(x)$, is a strongly interacting Bose field with asymptotic free Boson $\phi_{ \pm}^{F}(x)$. Their $S$-matrices are shown to coincide with $(-)^{N(N-1) / 2}$ in the $N$-particle sector ([4], [9]). The off-shell n-point functions themselves have simple relations to each other, both admitting exact expressions as in the complex case $([5],[9])$. In particular, corresponding to (4.0.4), we have the following ([9]).

$$
\left\langle\varphi_{B}\left(a_{1}\right) \cdots \varphi_{B}\left(a_{n}\right)\right\rangle\left\langle\varphi_{F}\left(a_{1}\right) \cdots \varphi_{F^{\prime}}\left(a_{n}\right)\right\rangle=\sqrt{\operatorname{det}} \overline{\cosh H}
$$

Here $H$ is related to a solution of the deformation equations.

We wish to thank Doctor K. R. Ito for informing us of the paper of Bariev [14]. We are also indebted to Professor M. Suzulki for showing us the preprints of the work [11], [12] by $\mathrm{Wu}$ et al.

\section{§ 4. 1. Construction of $\varphi_{B}$}

We denote by $X^{\text {Min }}$ the $n$-dimensional Minkowski space with the inner product $x^{2}=\left(x_{0}\right)^{2}-\left(x_{1}\right)^{2}-\cdots-\left(x_{n-1}\right)^{2}$ for $x=\left(x_{0}, \vec{x}\right)=\left(x_{0}, x_{1}, \cdots, x_{n-1}\right)$ $\in X^{\mathrm{Min}}$. We set $\partial_{\mu}=\partial / \partial x_{\mu} \quad(\mu=0,1, \cdots, n-1)$. We denote by $P$ the dual vector space of $X^{\mathrm{Min}}$. We choose the coordinate $p=\left(p_{0}, \vec{p}\right)=\left(p_{0}\right.$, 
$\left.p_{1}, \cdots, p_{n-1}\right)$ so that the bilinear form between $X^{\text {Min }}$ and $P$ reads $x \cdot p$ $=x_{0} p_{0}-\vec{x} \cdot \vec{p}=x_{0} p_{0}-x_{1} p_{1}-\cdots-x_{n-1} p_{n-1}$.

Consider the $n$-dimensional Klein-Gordon equation

$$
\left(\partial_{0}^{2}-\partial_{1}^{2}-\cdots-\partial_{n-1}^{2}+m^{2}\right) v(x)=0 .
$$

If $v$ and $v^{\prime}$ satisfy (4.1.1), then

$$
\begin{aligned}
& \mathcal{G}_{B}\left(v, v^{\prime}\right)=\left(v(x) \cdot \partial_{0} v^{\prime}(x)-\partial_{0} v(x) \cdot v^{\prime}(x)\right) d x_{1} \wedge \cdots \wedge d x_{n-1} \\
& +\sum_{j=1}^{n-1}(-)^{j-1}\left(v(x) \cdot \partial_{j} v^{\prime}(x)-\partial_{j} v(x) \cdot v^{\prime}(x)\right) d x_{0} \wedge \stackrel{\stackrel{j}{\sim}}{\cdots} \wedge d x_{n-1}
\end{aligned}
$$

is a closed $(n-1)$-form. Hence the inner product

$$
\left\langle v, v^{\prime}\right\rangle=-i \int_{r} \mathcal{I}_{B}\left(v, v^{\prime}\right)
$$

$r:$ spacelike

is independent of $\gamma^{(*)}$

We define the Fourier transformation by

$$
\begin{aligned}
& v(x)=\int \frac{d^{n} p}{(2 \pi)^{n}} \widehat{v}(p) e^{-i x \cdot p} \\
& \widehat{v}(p)=\int d^{n} x v(x) e^{i x \cdot p}
\end{aligned}
$$

(4.1.1) is transformed into $\left(p^{2}-m^{2}\right) \widehat{v}(p)=0$. Hence $\widehat{v}(p)$ is of the form $4 \pi \delta\left(p^{2}-m^{2}\right) v(p)$ where $v(p)$ is defined on the mass shell $M=\{p$ $\left.\in P \mid p^{2}-m^{2}=0\right\}$. We shall abbreviate the Lorentz invariant measure and the delta function on $M$ as follows.

$$
d \underline{p}=\frac{1}{\left|p_{0}\right|} \frac{d^{n-1} \vec{p}}{(2 \pi)^{n-1}}, \quad \delta\left(p, p^{\prime}\right)=\frac{\left|p_{0}+p_{0}^{\prime}\right|}{2}(2 \pi)^{n-1} \delta\left(\vec{p}-\vec{p}^{\prime}\right) .
$$

Now (4.1.3) reads

$$
\left\langle v, v^{\prime}\right\rangle=2 \int_{M} d p \varepsilon\left(p_{0}\right) v(p) v^{\prime}(-p)
$$

We call $v(x)$ (resp. $v(p)$ ) the $x$-(resp. $p$-) representation of $v$. The relation between these two representations is given by

(*) A $C^{1}$-hypersurface $r$ is called spacelike if for some $\varepsilon>0, r \cap\left\{x \in X^{\operatorname{Min}}|| x_{0}|\geqq(1-\varepsilon)| \vec{x} \mid\right\}$ is compact. 
(4.1.8) $\quad v(x)=\int_{M} d p v(p) e^{-i x \cdot p}$,

(4.1.9) $\quad v(p)=\frac{1}{2} \int_{x_{0}=\text { const. }} d^{n-1} \vec{x}\left(\left|p_{0}\right| v(x)+i \varepsilon\left(p_{0}\right) \partial_{0} v(x)\right) e^{i x \cdot p}$.

We denote by $W_{\boldsymbol{B}}^{\boldsymbol{R}}$ the set of real-valued solutions of (4.1.1) satisfying $\int \underline{d p}|v(p)|^{2}<\infty$, and set $W_{B}=W^{\mathbb{R}} \underset{\boldsymbol{R}}{\otimes}$. $\quad W_{B}$ equipped with the antisymmetric inner product $\left\langle v, v^{\prime}\right\rangle$ is a symplectic vector space.

We set

$$
\begin{aligned}
& V_{B}^{\dot{\dagger}}=\left\{v \in W_{B} \mid v(p) \equiv 0 \quad \text { for } \quad p \in M_{-}\right\}, \\
& V_{B}=\left\{v \in W_{B} \mid v(p) \equiv 0 \quad \text { for } \quad p \in M_{+}\right\} .
\end{aligned}
$$

where $M_{ \pm}=\left\{p \in M \mid p_{0} \gtrless 0\right\} . \quad W_{B}=V_{B}^{\dagger} \oplus V_{B}$ is a holonomic decomposition. In the sequel we mean by $\mathrm{Nr}$ or $\langle>$ the norm or the vacuum expectation value with respect to this holonomic decomposition. See Appendix as for generalities on expectation value, norm, etc. in the symplectic case.

Let $\left(X^{\text {Min }}\right) *$ denote the set of ordered pairs of null hyperplanes in $X^{\mathrm{Min}}$ which are not parallel to each other. $\left(X^{\mathrm{Min}}\right) *$ is of $2(n-1)$ dimensions. We denote by $\langle\xi, r\rangle$ the null hyperplane $\left\{x \in X^{\operatorname{Min}} \mid x \cdot \xi=r\right\}$, where $\xi \in P$ is positively lightlike ${ }^{(*)}$ vector and $r \in \mathbb{R}$. We note that $\langle c \xi, c r\rangle=\langle\xi, r\rangle$ for $c\rangle 0$. Let $a^{*}=\left(\left\langle\xi_{+}, r_{+}\right\rangle,\left\langle\xi_{-}, r_{-}\right\rangle\right) \in\left(X^{\mathrm{Min}}\right) *$ and set

$$
\begin{gathered}
W_{B}^{ \pm}\left(a^{*}\right)=\left\{v \in W_{B} \mid v(x) \equiv 0 \text { if } x \cdot \xi_{+}-r_{+} \lessgtr 0\right. \\
\text { and } \left.x \cdot \xi_{-}-r_{-} \gtrless 0\right\} .
\end{gathered}
$$

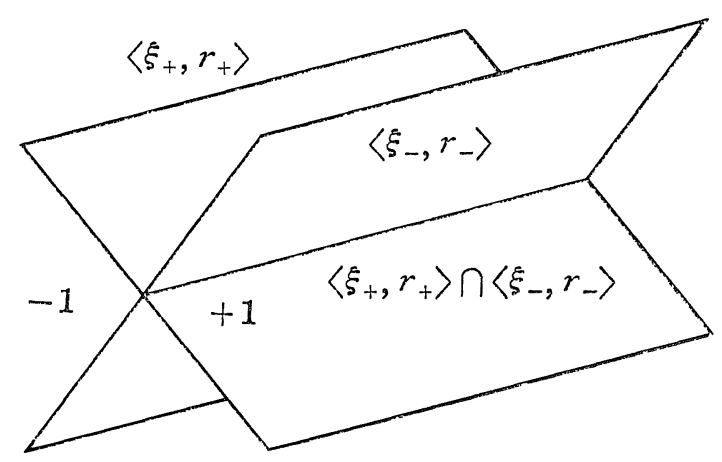

Figure 4.1

(*) $\xi_{0}>0, \xi^{2}=0$. 
Then we have $W_{B}=W_{B}^{+}\left(a^{*}\right) \oplus W_{\bar{B}}^{-}\left(a^{*}\right)$. We define a symplectic transformation ( $=\mathrm{a}$ linear transformation which preserves the inner product) $T_{a^{*}}$ by

$$
T_{a^{*}}\left(v^{+}+v^{-}\right)=v^{+}-v^{-} \quad \text { for } \quad v^{ \pm} \in W_{B}^{ \pm}\left(a^{*}\right) .
$$

We shall compute the norm of the operator $\varphi_{B}\left(a^{*}\right)$ which induces this symplectic transformation. (See Figure 4.1.)

Remark. The intersection $\left\langle\xi_{+}, r_{+}\right\rangle \cap\left\langle\xi_{-}, r_{-}\right\rangle$is a spacelike linear submanifold of codimensions 2. Conversely, there is a unique pair of null hyperplanes which pass a given spacelike linear submanifold of codimensions 2. Thus $\left(X^{\mathrm{Min}}\right) *$ is identified with the set of oriented spacelike linear submanifolds of codimensions 2 . If $n=2\left(X^{\mathrm{Min}}\right) *$ is nothing but the disjoint union of two copies of $X^{\mathrm{Min}}$.

Before the computation of the norm we prepare generalities on free fields. We denote by $\phi(y)\left(y \in X^{\mathrm{Min}}\right)$ the solution $v(x ; y)$ of $(4.1 .1)$ which satisfies

$$
\left.v(x ; y)\right|_{x_{0}=y_{0}}=0,\left.\quad \frac{\partial v}{\partial x_{0}}(x, y)\right|_{x_{0}=y_{0}}=-\sqrt{2} i \delta\left(\begin{array}{ll}
\vec{x} & \vec{y}
\end{array}\right) .
$$

We have

$$
v(x ; y)=\frac{-i}{\sqrt{2}} \Delta\left(x-y ; m^{2}\right),
$$

where

$$
\begin{aligned}
& \Delta\left(x ; m^{2}\right)=i \int_{M} \frac{d p}{p} \varepsilon\left(p_{0}\right) e^{-i x \cdot p} \\
& = \begin{cases}\varepsilon\left(x_{0}\right)\left(\frac{m}{2 \pi \sqrt{ } x^{2}}\right)^{(n / 2)-1} J_{1-n / 2}\left(m \sqrt{x^{2}}\right) & \left(x^{2}>0\right) \\
0 & \left(x^{2}<0\right) .\end{cases}
\end{aligned}
$$

The $p$ representation of $\phi(y)$ is

$$
v(p ; y)=\frac{1}{\sqrt{2}} \varepsilon\left(p_{0}\right) e^{i y \cdot p}
$$

$\phi(x)$ satisfies (4.1.1) with respect to $x$. 
(4.1.18)

$$
\left(\partial_{0}^{2}-\partial_{1}^{2}-\cdots-\partial_{n-1}^{2}+m^{2}\right) \phi(x)=0 \text {. }
$$

We set

$$
\pi(x)=\frac{\partial}{\partial x_{0}} \phi(x) .
$$

(4.1.14) and (4.1.19) imply that

$$
v=\frac{i}{\sqrt{2}} \int_{x_{0}=\text { const. }} d^{n-1} \vec{x}\left(\partial_{0} v(x) \cdot \phi(x)-v(x) \cdot \pi(x)\right)
$$

for $v \in W$. Conversely we have

$$
v(x)=\frac{1}{\sqrt{2}}\langle\phi(x), v\rangle .
$$

The table of inner product of $\phi(x)$ 's read

$$
\left\langle\phi(x), \phi\left(x^{\prime}\right)\right\rangle=-i \Delta\left(x-x^{\prime} ; m^{2}\right) .
$$

At equal times it reduces to

$$
\begin{aligned}
&\left(\begin{array}{cc}
\left\langle\phi(x), \phi\left(x^{\prime}\right)\right\rangle & \left\langle\phi(x), \pi\left(x^{\prime}\right)\right\rangle \\
\left\langle\pi(x), \phi\left(x^{\prime}\right)\right\rangle & \left\langle\pi(x), \pi\left(x^{\prime}\right)\right\rangle
\end{array}\right)_{\mid x_{0}=x_{0}^{\prime}} \\
&=\left(\begin{array}{cc}
0 & 2 i \delta\left(\vec{x}-\vec{x}^{\prime}\right) \\
-2 i \delta\left(\vec{x}-\vec{x}^{\prime}\right) & 0
\end{array}\right) .
\end{aligned}
$$

Next let $\phi(q) \quad(q \in M)$ denote the solution $v(x ; q)$ of (4.1.1) given by

$$
v(x ; q)=\frac{-1}{\sqrt{2}} \varepsilon\left(q_{0}\right) e^{i x \cdot p} .
$$

The $p$ representation of $\phi(q)$ is given by

$$
v(p ; q)=\frac{\varepsilon\left(p_{0}\right)}{\sqrt{2}} \underline{\delta}(p,-q) \text {. }
$$

These special elements $\phi(x), \pi(x)$ and $\phi(q)$ are interrelated through

$$
\phi(x)=\int d \underline{p} \phi(p) e^{-i x \cdot p}
$$

$$
\phi(p)=\frac{1}{2} \int_{x_{0}=\text { const. }} d^{n-1} \vec{x}\left(\left|p_{0}\right| \phi(x)+i \varepsilon\left(p_{0}\right) \pi(x)\right) e^{i x \cdot p} .
$$

The table of inner product of $\phi(p)$ 's reads 


$$
\left\langle\phi(p), \phi\left(p^{\prime}\right)\right\rangle=\varepsilon\left(p_{0}\right) \underline{\delta}\left(p,-p^{\prime}\right)
$$

Similarly to (4.1.20) and (4.1.21) we have

$$
v=-\sqrt{2} \int \underline{d p} \varepsilon\left(p_{0}\right) v(-p) \phi(p)
$$

$$
v(p)=\frac{1}{\sqrt{2}}\langle\phi(p), v\rangle
$$

As in Section 2.1 we consider $\phi(x), \pi(x)$ and $\phi(p)$ to be ideal elements of $W_{B}$ and construct the operator theory. Then $\phi(x)$ is nothing but the free Bose field operator and $\phi(p)$ the creation $\left(p^{0}<0\right)$-annihilation $\left(p^{0}>0\right)$ operator thereof. In this context (4.1.22) and (4.1.28) mean the following commutation relations.

$$
\left[\phi(x), \phi\left(x^{\prime}\right)\right]=-i \Delta\left(x-x^{\prime} ; m^{2}\right),
$$

$$
\left[\phi(p), \phi\left(p^{\prime}\right)\right]=\varepsilon\left(p_{0}\right) \underline{\delta}\left(p,-p^{\prime}\right) .
$$

The tables of vacuum expectation values read

$$
\begin{aligned}
\left\langle\phi(p) \phi\left(p^{\prime}\right)\right\rangle & =\theta\left(p_{0}\right) \underline{o}\left(p,-p^{\prime}\right), \\
\left\langle\phi(x) \phi\left(x^{\prime}\right)\right\rangle & =\int \underline{d p} \theta\left(p_{0}\right) e^{-i\left(x-x^{\prime}\right) \cdot p} \\
& =-i \Delta^{(+)}\left(x-x^{\prime} ; m^{2}\right),
\end{aligned}
$$

where

$$
\begin{aligned}
& d^{(+)}\left(x ; m^{2}\right)=i \int d p \theta\left(p_{0}\right) e^{-i x \cdot p} \\
& =\left(\frac{m}{2 \pi \sqrt{-\left(x_{0}-i 0\right)^{2}+\vec{x}^{2}}}\right)^{(n / 2)-1} \cdot \frac{i}{\pi} K_{1-n / 2}\left(m \sqrt{-\left(x_{0}-i 0\right)^{2}+\vec{x}^{2}}\right) .
\end{aligned}
$$

Let $g \in S O_{0}(1, n-1)$ and $a \in \boldsymbol{R}^{n}$. The action of the Poincaré group $S O_{0}(1, n-1) \times \boldsymbol{R}^{n}=\left\{\widetilde{g}=(g, a) \mid g \in S O_{0}(1, n-1), a \in \boldsymbol{R}^{n}\right\}$ is given by $\tilde{g} \cdot x$ $=g \cdot x+a$, and the representation $\varpi$ of the Poincare group in $W_{B}$ is given by

$$
\left(\varpi_{B}(\widetilde{g}) v\right)(x)=v\left(\widetilde{g}^{-1} x\right)
$$

Then we have

$$
\varpi_{B}(\widetilde{g}) \phi(x)=\phi(\widetilde{g} x),
$$




$$
\widetilde{\varpi}_{B}(\widetilde{g}) \operatorname{grad} \phi(x)=(\operatorname{grad} \phi)(\widetilde{g} x) \cdot g
$$

where $\operatorname{grad} \phi=\left(\partial_{0} \phi, \partial_{1} \phi, \cdots, \partial_{n-1} \phi\right)$, and

$$
\varpi_{B}(\widetilde{g}) \phi(p)=\phi(g p) e^{-i a \cdot y p} .
$$

The vacuum expectation value and the inner product are invariant under this action of the Poincaré group.

Now, applying Proposition A. 1 in the Appendix we shall compute $\operatorname{Nr}\left(\varphi_{B}\left(a^{*}\right)\right)=\exp \left(\rho_{B}\left(a^{*}\right) / 2\right)$ so that $\varphi_{B}\left(a^{*}\right)$ induces the symplectic transformation (4.1.13).

From (4.1.16) $\phi(x)$ belongs to $W_{B}^{+}\left(a^{*}\right)$ (resp. $\left.W_{\bar{B}}^{-}\left(a^{*}\right)\right)$ if $x \cdot \xi_{+}-r_{+}>0$ and $x \cdot \xi_{-}-r_{-}<0$ (resp. $x \cdot \xi_{+}-r_{+}<0$ and $x \cdot \xi_{-}-r_{-}>0$ ). Hence (4.1.13) implies the following commutation relations between $\varphi_{B}\left(a^{*}\right)$ and $\phi(x)$.

$$
\text { (4. 1. 38) } \varphi_{B}\left(a^{*}\right) \phi(x)= \begin{cases}\phi(x) \varphi_{B}\left(a^{*}\right) \text { if } x \cdot \xi_{+}-r_{+}>0 \\ & \text { and } x \cdot \xi_{-}-r_{-}<0 \\ -\phi(x) \varphi_{B}\left(a^{*}\right) & \text { if } x \cdot \xi_{+}-r_{+}<0 \\ & \text { and } x \cdot \xi_{-}-r_{-}>0\end{cases}
$$

Because of the Poincare covariance of our theory, we may assume without loss of generality that

$$
\xi_{上}=(1, \mp 1,0, \cdots, 0), r_{\leq}=0 \text {. }
$$

First we define auxiliary basis $\hat{\phi}(\vec{p}), \hat{\pi}(\vec{p})$ by

$$
\begin{aligned}
& \hat{\phi}(\vec{p})=\int d^{n-1} \vec{x} \phi(0, \vec{x}) e^{-i \vec{x} \cdot \vec{p}}, \\
& \hat{\pi}(\vec{p})=\int d^{n-1} \vec{x} \pi(0, \vec{x}) e^{-\overrightarrow{i x} \cdot \vec{p}},
\end{aligned}
$$

or equivalently, by

$$
\widehat{\phi}(\vec{p})=\frac{1}{\omega(\vec{p})}\{\phi(\omega(\vec{p}), \vec{p})+\phi(-\omega(\vec{p}), \vec{p})\}
$$

$$
\widehat{\pi}(\vec{p})=-i\{\phi(\omega(\vec{p}), \vec{p})-\phi(-\omega(\vec{p}), \vec{p})\},
$$

where we have set

$$
\omega(\vec{p})=\sqrt{ } \vec{p}^{2}+m^{2}
$$

In what follows we identify a kernel function $F\left(\vec{p}, \vec{p}^{\prime}\right)$ 
$=\left(\begin{array}{ll}F_{1}\left(\vec{p}, \vec{p}^{\prime}\right) & F_{2}\left(\vec{p}, \vec{p}^{\prime}\right) \\ F_{3}\left(\vec{p}, \vec{p}^{\prime}\right) & F_{4}\left(\vec{p}, \vec{p}^{\prime}\right)\end{array}\right)$ with the associated linear transformation $F$ given by

$$
\left(F \hat{\phi}\left(\vec{p}^{\prime}\right), F \hat{\pi}\left(\vec{p}^{\prime}\right)\right)=\int \frac{d^{n-1} \vec{p}}{(2 \pi)^{n-1}}(\hat{\phi}(\vec{p}), \hat{\pi}(\vec{p})) F\left(\vec{p}, \vec{p}^{\prime}\right) .
$$

With respect to $\hat{\phi}(\vec{p})$ 's and $\hat{\pi}(\vec{p})$ 's the above mentioned kernel representation of $\left(1-T_{a^{*}}\right) / 2$ is given by

$$
P\left(\vec{p}, \vec{p}^{\prime}\right)=\frac{-i}{p_{1}-p_{1}^{\prime}-i 0}(2 \pi)^{n-2} \delta\left(p^{\perp}-p^{\prime \perp}\right) I_{2}
$$

where we have set

$$
p^{\perp}=\left(p_{2}, \cdots, p_{n-1}\right)
$$

The table of vacuum expectation values with respect to the basis $\hat{\phi}(\vec{p}), \hat{\pi}(\vec{p})$ reads

$$
\begin{aligned}
K\left(\vec{p}, \vec{p}^{\prime}\right) & =\left(\begin{array}{cc}
\left\langle\hat{\phi}(\vec{p}) \hat{\phi}\left(\vec{p}^{\prime}\right)\right\rangle\left\langle\hat{\phi}(\vec{p}) \hat{\pi}\left(\vec{p}^{\prime}\right)\right\rangle \\
\left\langle\hat{\pi}(\vec{p}) \hat{\phi}\left(\vec{p}^{\prime}\right)\right\rangle\left\langle\hat{\pi}(\vec{p}) \hat{\pi}\left(\vec{p}^{\prime}\right)\right\rangle
\end{array}\right) \\
& =\left(\begin{array}{cc}
\frac{1}{\omega(\vec{p})} & i \\
-i & \omega(\vec{p})
\end{array}\right)(2 \pi)^{n-1} \delta\left(\vec{p}+\vec{p}^{\prime}\right) .
\end{aligned}
$$

Hence $H$ in (A. 8) and $E$ in (A. 17) have the kernels

$$
H\left(\vec{p}, \vec{p}^{\prime}\right)=\left(\begin{array}{c}
2 i \\
-2 i
\end{array}\right)(2 \pi)^{n-1} \delta\left(\vec{p}+\vec{p}^{\prime}\right),
$$

$$
E\left(\vec{p}, \vec{p}^{\prime}\right)=i\left(\begin{array}{c}
\omega(\vec{p}) \\
\frac{-1}{\omega(\vec{p})}
\end{array}\right)(2 \pi)^{n-1} \delta\left(\vec{p}-\vec{p}^{\prime}\right) .
$$

We set

$$
\mu(\vec{p})=\sqrt{\left(p^{\perp}\right)^{2}+m^{2}} .
$$

$X_{ \pm}$of Proposition A. 1 are given by

(4. 1. 51) $\quad X_{+}\left(\vec{p}, \vec{p}^{\prime}\right)=\left(\begin{array}{c}{\sqrt{p_{1}+i \mu(\vec{p})}}^{-1} \\ {\sqrt{p_{1}+i \mu(\vec{p})}}^{-1}\end{array}\right)(2 \pi)^{n-1} \delta\left(\vec{p}-\vec{p}^{\prime}\right)$,

(4.1.52) $\quad X_{-}\left(\vec{p}, \vec{p}^{\prime}\right)=i\left({\sqrt{p_{1}-i \mu(\vec{p})}}^{-1}{ }^{p_{1}-i \mu(\vec{p})}\right)(2 \pi)^{n-1} \delta\left(\vec{p}-\vec{p}^{\prime}\right)$.

Hence by (A. 19) we have 
(4. 1.53) $R\left(\vec{p}, \vec{p}^{\prime}\right)$

$$
\begin{gathered}
=\left(\begin{array}{c}
-\sqrt{p_{1}-i \mu(\vec{p})} \sqrt{p_{1}^{\prime}-i \mu\left(\vec{p}^{\prime}\right)} \\
\frac{1}{\sqrt{p_{1}-i \mu(\vec{p})} \sqrt{p_{1}^{\prime}-i \mu\left(\vec{p}^{\prime}\right)}}
\end{array}\right) \\
\times \frac{(2 \pi)^{n-2} \delta\left(p^{\perp}+p^{\perp^{\prime}}\right)}{p_{1}+p_{1}^{\prime}-i 0} .
\end{gathered}
$$

We set

$$
u^{ \pm 1}=\frac{p_{0} \pm p_{1}}{\mu(\vec{p})} \quad(p \in M)
$$

Then we have $\sqrt{p_{1} \pm i \mu(\vec{p})}=\sqrt{\frac{\mu(\vec{p})}{2}}(\sqrt{u} \pm i \sqrt{u}-1)\left(\operatorname{resp} \cdot \sqrt{\frac{\mu(\vec{p})}{2}}\left(\sqrt{u^{-1}}\right.\right.$ $\pm i \sqrt{-\bar{u}})$ ) for $u>0$ (resp. $u<0$ ). From (4.1.41), (4.1.42) and (4. 1.53) we have

$$
\begin{aligned}
\rho_{B}\left(a^{*}\right)= & \iint \frac{d p d p^{\prime}}{\frac{-2 \sqrt{u-i 0} \sqrt{u^{\prime}-i 0}}{u+u^{\prime}-i 0}} \\
& \times(2 \pi)^{n-2} \delta\left(p^{\perp}+p^{\prime \perp}\right) \phi(p) \phi\left(p^{\prime}\right) .
\end{aligned}
$$

Finally, using Poincaré covariance, we rewrite (4.1.55) in a general form.

Let $P_{a^{*}}$ be the subspace of $P$ spanned by $\xi_{+}$and $\xi_{-}$, and let $P_{a^{*}}^{\perp}$ be its orthogonal complement. We denote by $p_{a^{*}}$ (resp. $p_{a^{*}}^{\perp}$ ) the $P_{a^{*}}$ (resp. $\left.P_{a^{*}}^{\perp}\right)$ component of $p \in P$.

$$
p=p_{a^{*}}+p_{a^{*}}^{\perp}, \quad p_{a^{*}} \in P_{a^{*}}, \quad p_{a^{*}}^{\perp} \in P_{a^{*}}^{\perp} .
$$

Let $x\left(a^{*}\right)$ be a vector in $X^{\text {Min }}$ satisfying

$$
x\left(a^{*}\right) \cdot \xi_{ \pm}=r_{ \pm} .
$$

Theorem 4. 1. 1. Normalizing $\varphi_{B}\left(a^{*}\right)$ so that $\left\langle\varphi_{B}\left(a^{*}\right)\right\rangle=1$, we have

$$
\operatorname{Nr}\left(\varphi_{B}\left(a^{*}\right)\right)=\exp \left(\rho_{B}\left(a^{*}\right) / 2\right)
$$

where

$$
\rho_{B}\left(a^{*}\right)=\iint \frac{d p d p^{\prime}-2 \sqrt{p \cdot \xi_{+}-i 0} \sqrt{p^{\prime} \cdot \xi_{+}-i 0}}{p \cdot \xi_{+}+p^{\prime} \cdot \xi_{+}-i 0}(2 \pi)^{n-2} \delta\left(p_{a^{*}}^{\perp}+p_{p^{*}}^{\prime \frac{1}{*}}\right)
$$




$$
\times e^{-i x\left(a^{2}\right)\left(p+p^{\prime}\right)} \phi(p) \phi\left(p^{\prime}\right) .
$$

In the sequel, we restrict ourselves to the case $n=2$. We identify $X^{\mathrm{Min}}$ with a component of $\left(X^{\mathrm{Min}}\right) *$ by

\section{(4. 1. 59)}

$$
a=\left(a_{0}, a_{1}\right) \in X^{\mathrm{Min}} \leftrightarrow\left(\left\langle(1,-1), a_{0}+a_{1}\right\rangle,\left\langle(1,1), a_{0}-a_{1}\right\rangle\right) \in\left(X^{\mathrm{Min}}\right) * .
$$

It is convenient to use characteristic coordinates

$$
x^{x}=\left(x_{0} \pm x_{1}\right) / 2 .
$$

(4. 1. 54) reduces to

$$
u^{ \pm 1}=\left(p_{0} \pm p_{1}\right) / m \quad(p \in M) .
$$

$u$ serves as a coordinate on $M$, and we write $\phi(u)$ instead of $\phi(p)$. The invariant volume $d p$ is equal to

$$
d u=\frac{d u}{2 \pi|u|}
$$

The tables of vacuum expectation values and inner products for $\phi(u)$ read

$$
\left\langle\phi(u) \phi\left(u^{\prime}\right)\right\rangle=2 \pi u u_{+} \delta\left(u+u^{\prime}\right),
$$

$$
\left\langle\phi(u), \phi\left(u^{\prime}\right)\right\rangle=2 \pi u \delta\left(u+u^{\prime}\right) .
$$

(4. 1. 26) and (4.1.58) reduce to

$$
\phi(x)=\int d u \phi(u) e^{-i m\left(x^{-} u+x^{+} u^{-1}\right)},
$$

$$
\operatorname{Nr}\left(\varphi_{B}(a)\right)=\exp \left(\rho_{B}(a) / 2\right),
$$

where

$$
\begin{aligned}
\rho_{B}(a)=\iint \frac{d u d u^{\prime}-2 \sqrt{u-i 0} \sqrt{u^{\prime}-i 0}}{u+u^{\prime}-i 0} \\
\\
\quad \times e^{-i m\left(a-\left(u+u^{\prime}\right)+a^{+}\left(u^{-1}+u^{\prime-1}\right)\right)} \phi(u) \phi\left(u^{\prime}\right) .
\end{aligned}
$$

\section{$\S$ 4. 2. Construction of $\varphi_{F}$}

In this section we restrict ourselves to the case $\operatorname{dim} X^{\mathrm{Min}}=2$ or 3 . First we consider the case $\operatorname{dim} X^{\mathrm{Min}}=3$. 
Consider the 3-dimensional Dirac equation

$$
\left(\begin{array}{cc}
m-\partial_{2} & \partial_{0}-\partial_{1} \\
-\partial_{0}-\partial_{1} & m+\partial_{2}
\end{array}\right)\left(\begin{array}{l}
w_{+}(x) \\
w_{-}(x)
\end{array}\right)=0 .
$$

If $w=\left(\begin{array}{c}w_{+}(x) \\ w_{-}(x)\end{array}\right)$ and $w^{\prime}=\left(\begin{array}{c}w_{+}^{\prime}(x) \\ w_{-}^{\prime}(x)\end{array}\right)$ satisfy $(4.2 .1)$, then

(4. 2. 2) $\mathcal{J}_{F}\left(w, w^{\prime}\right)=\left(w_{+}(x) w_{+}^{\prime}(x)+w_{-}(x) w_{-}^{\prime}(x)\right) d x_{1} \wedge d x_{2}$

$$
\begin{aligned}
& -\left(w_{+}(x) w_{+}^{\prime}(x)-w_{-}(x) w_{-}^{\prime}(x)\right) d x_{0} \wedge d x_{2} \\
& -\left(w_{+}(x) w_{-}^{\prime}(x)+w_{-}(x) w_{+}^{\prime}(x)\right) d x_{0} \wedge d x_{1}
\end{aligned}
$$

is a closed 2-form. Hence the inner product

$$
\left\langle w, w^{\prime}\right\rangle=\frac{m}{2} \int_{r} \mathcal{I}_{F}\left(w, w^{\prime}\right)
$$

$$
\text { r: spacelike }
$$

is independent of $\gamma$.

We denote by $W_{F}^{\boldsymbol{R}}$ the set of real-valued solutions of (4.2.1) satisfying $\langle w, w\rangle\left\langle\infty\right.$, and set $W_{F}=W_{F}^{\mathbb{R}} \bigotimes_{\boldsymbol{R}} \boldsymbol{C} . \quad W_{F}$ equipped with the inner product $\left\langle w, w^{\prime}\right\rangle$ is an orthogonal vector space.

By the Fourier transformation (4.1.4) and (4.1.5), (4.2.1) is transformed into

$$
\left(\begin{array}{cc}
m-i p_{2} & -i\left(p_{0}+p_{1}\right) \\
i\left(p_{0}-p_{1}\right) & m+i p_{2}
\end{array}\right)\left(\begin{array}{c}
\widehat{w}_{+}(p) \\
\widehat{\mho}_{-}(p)
\end{array}\right)=0 .
$$

Hence $\left(\begin{array}{l}\widehat{w}_{+}(p) \\ \widehat{w}_{-}(p)\end{array}\right)$ is of the form

$$
\begin{aligned}
\left(\begin{array}{l}
\widehat{w}_{+}(p) \\
\widehat{w}_{-}(p)
\end{array}\right)= & 4 \pi \delta\left(p^{2}-m^{2}\right)\left(\frac{\sqrt{m^{2}+p_{2}^{2}}}{m}\right)^{\frac{1}{2}} \\
& \times\left(\begin{array}{l}
\sqrt{\frac{0+i\left(p_{0}+p_{1}\right)}{m-i p_{2}}} \\
\sqrt{\frac{0-i\left(p_{0}-p_{1}\right)}{m+i p_{2}}}
\end{array}\right) w(p)
\end{aligned}
$$

where $w(p)$ is defined on the mass shell.

(4. 2.3) reads

$$
\left\langle w, w^{\prime}\right\rangle=\int d \underline{d p} w(p) w^{\prime}(-p) .
$$


We call $\left(\begin{array}{c}w_{+}(x) \\ w_{-}(x)\end{array}\right)$ (resp. $w(p)$ ) the $x$-(resp. $p$-) representation of $w$. The relation between these two representations is given by

$$
\left(\begin{array}{l}
w_{+}(x) \\
w_{-}(x)
\end{array}\right)=\int \frac{d p}{\left(\frac{\sqrt{m^{2}+p_{2}^{2}}}{m}\right)^{\frac{1}{2}}}\left(\begin{array}{l}
\sqrt{\frac{0+i\left(p_{0}+p_{1}\right)}{m-i p_{2}}} \\
\sqrt{\frac{0-i\left(p_{0}-p_{1}\right)}{m+i p_{2}}}
\end{array}\right) w(p) e^{-i x \cdot p}
$$

(4. 2. 8) $\quad w(p)=\frac{m}{2} \int_{x_{0}=\text { const. }} d^{2} \vec{x}\left(\frac{\sqrt{m^{2}+p_{2}^{2}}}{m}\right)^{\frac{1}{2}}$

$$
\times\left(\sqrt{\frac{0-i\left(p_{0}+p_{1}\right)}{m+i p_{2}}} w_{+}(x)+\sqrt{\frac{0+i\left(p_{0}-p_{1}\right)}{m-i p_{2}}} w_{-}(x)\right) e^{i x \cdot p} .
$$

A holonomic decomposition is given by $W_{F}=V_{F}^{\dagger} \oplus V_{F}$, where

$$
V_{F}^{\dagger}=\left\{w \in W_{F} \mid w(p) \equiv 0 \quad \text { for } \quad p \in M_{-}\right\}
$$

$$
V_{F}=\left\{w \in W_{F} \mid w(p) \equiv 0 \quad \text { for } \quad p \in M_{+}\right\} \text {. }
$$

An orthogonal transformation $T_{a^{*}}$ is given by

$$
T_{a^{*}}\left(w^{+}+w^{-}\right)=w^{+}-w^{-} \quad \text { for } \quad w^{ \pm} \in W_{F}^{ \pm}\left(a^{*}\right),
$$

where

$$
\begin{aligned}
& W_{F}^{ \pm}\left(a^{*}\right)=\left\{w \in W_{F} \mid w_{+}(x) \equiv w_{-}(x) \equiv 0\right. \\
& \left.\quad \text { if } x \cdot \xi_{+}-r_{+} \lessgtr 0 \text { and } x \cdot \xi_{-}-r_{-} \gtrless 0\right\} .
\end{aligned}
$$

We shall compute the norm of $\varphi_{F}\left(a^{*}\right)$ which induces the orthogonal transformation (4.2.11).

We denote by $\psi_{+}(y)$ (resp. $\left.\psi_{-}(y)\right)$ the solution $\left(\begin{array}{l}w_{++}(x ; y) \\ w_{+-}(x ; y)\end{array}\right)($ resp. $\left.\left(\begin{array}{l}w_{-+}(x ; y) \\ w_{--}(x ; y)\end{array}\right)\right)$ of $(4.2 .1)$ which satisfy

$$
\begin{aligned}
& \left.\left(\begin{array}{c}
w_{++}(x ; y) \\
w_{+-}(x ; y)
\end{array}\right)\right|_{x_{0}=y_{0}}=\frac{2}{m}\left(\begin{array}{c}
\delta(\vec{x}-\vec{y}) \\
0
\end{array}\right) \\
& \quad\left(\left.\operatorname{resp.}\left(\begin{array}{l}
w_{-+}(x ; y) \\
w_{--}(x ; y)
\end{array}\right)\right|_{x_{0}=y_{0}}=\frac{2}{m}\left(\begin{array}{c}
0 \\
\delta(\vec{x}-\vec{y})
\end{array}\right)\right) .
\end{aligned}
$$

We have

$$
\left(\begin{array}{ll}
w_{++}(x ; y) & w_{-+}(x ; y) \\
w_{+-}(x ; y) & w_{--}(x ; y)
\end{array}\right)
$$




$$
=\frac{1}{m}\left(\begin{array}{cc}
\partial_{0}-\partial_{1} & m+\partial_{2} \\
-m+\partial_{2} & \partial_{0}+\partial_{1}
\end{array}\right) \Delta\left(x-y ; m^{2}\right) .
$$

The p-representation of $\psi_{+}(y)$ (resp. $\left.\psi_{-}(y)\right)$ is

$$
\begin{aligned}
& w_{+}(p ; y)=\left(\frac{\sqrt{m^{2}+p_{2}^{2}}}{m}\right)^{\frac{1}{2}} \sqrt{\frac{0-i\left(p_{0}+p_{1}\right)}{m+i p_{2}}} e^{i y \cdot p} \\
& \left(\text { resp. } w_{-}(p ; y)=\left(\frac{\sqrt{m^{2}+p_{2}^{2}}}{m}\right)^{\frac{1}{2}} \sqrt{\frac{0+i\left(p_{0}+p_{1}\right)}{m-i p_{2}}} e^{i y \cdot p}\right) .
\end{aligned}
$$

$\psi_{ \pm}(x)$ satisfy (4.2.1) with respect to $x$.

$$
\left(\begin{array}{cc}
m-\partial_{2} & \partial_{0}-\partial_{1} \\
-\partial_{0}-\partial_{1} & m+\partial_{2}
\end{array}\right)\left(\begin{array}{l}
\psi_{+}(x) \\
\psi_{-}(x)
\end{array}\right)=0 .
$$

(4. 2.13) implies that

(4. 2.17)

$$
w=\frac{m}{2} \int d^{2} \vec{x}\left(w_{+}(x) \psi_{+}(x)+w_{-}(x) \psi_{-}(x)\right) .
$$

Conversely we have

$$
w_{ \pm}(x)=\left\langle\psi_{ \pm}(x), w\right\rangle .
$$

We denote by $\psi(q)(q \in M)$ the solution $w_{ \pm}(x ; q)$ of $(4.2 .1)$ given by

$$
\left(\begin{array}{c}
w_{+}(x ; q) \\
w_{-}(x ; q)
\end{array}\right)=\left(\frac{\sqrt{m^{2}+q_{2}^{2}}}{m}\right)\left(\begin{array}{l}
\sqrt{\frac{0-i\left(q_{0}+q_{1}\right)}{m+i q_{2}}} \\
\sqrt{\frac{0+i\left(q_{0}-q_{1}\right)}{m-i q_{2}}}
\end{array}\right) e^{i x \cdot q} .
$$

The $p$ representation is given by

$$
w(p ; q)=\underline{\delta}(p,-q) .
$$

Similarly to (4.2.17) and (4.2.18) we have

$$
\begin{aligned}
& w=\int \underline{d p} w(-p) \psi(p), \\
& w(p)=\langle\psi(p), w\rangle .
\end{aligned}
$$

The relation between the basis $\psi_{ \pm}(x)$ and $\psi(p)$ reads

(4.2.23) $\left(\begin{array}{l}\psi_{+}(x) \\ \psi_{-}(x)\end{array}\right)=\int \frac{d p}{\left(\frac{\sqrt{m^{2}+p_{2}^{2}}}{m}\right)^{\frac{1}{2}}}\left(\sqrt{\frac{0+i\left(p_{0}+p_{1}\right)}{m-i p_{2}}}\right) \psi(p) e^{-i x \cdot p}$, 


\section{(4. 2. 24)}

$$
\begin{aligned}
\psi(p) & =\frac{m}{2} \int_{x_{0}=\text { const. }} d^{2} \vec{x}\left(\frac{\sqrt{m^{2}+p_{2}^{2}}}{m}\right)^{\frac{1}{2}} \\
& \times\left(\sqrt{\frac{0-i\left(p_{0}+p_{1}\right)}{m+i p_{2}}} \psi_{+}(x)+\sqrt{\frac{0+i\left(p_{0}-p_{1}\right)}{m-i p_{2}}} \psi_{-}(x)\right) e^{i x \cdot p} .
\end{aligned}
$$

The tables of vacuum expectation values and inner products are as follows.

$$
\left\langle\psi(p) \psi\left(p^{\prime}\right)\right\rangle=\theta\left(p_{0}\right) \underline{\delta}\left(p,-p^{\prime}\right),
$$

$$
\left\langle\psi(p), \psi\left(p^{\prime}\right)\right\rangle=\left[\psi(p), \psi\left(p^{\prime}\right)\right]_{+}=\underline{\delta}\left(p,-p^{\prime}\right),
$$

$$
\begin{aligned}
& \left(\begin{array}{l}
\left\langle\psi_{+}(x) \psi_{+}\left(x^{\prime}\right)\right\rangle\left\langle\psi_{+}(x) \psi_{-}\left(x^{\prime}\right)\right\rangle \\
\left\langle\psi_{-}(x) \psi_{+}\left(x^{\prime}\right)\right\rangle\left\langle\psi_{-}(x) \psi_{-}\left(x^{\prime}\right)\right\rangle
\end{array}\right) \\
& =\frac{1}{m}\left(\begin{array}{cc}
\partial_{0}-\partial_{1} & m+\partial_{2} \\
-m+\partial_{2} & \partial_{0}+\partial_{1}
\end{array}\right) \Delta^{(+)}\left(x-x^{\prime} ; m^{2}\right),
\end{aligned}
$$

$$
\begin{aligned}
\left(\begin{array}{l}
\left\langle\psi_{+}(x), \psi_{+}\left(x^{\prime}\right)\right\rangle\left\langle\psi_{+}(x), \psi_{-}\left(x^{\prime}\right)\right\rangle \\
\left\langle\psi_{-}(x), \psi_{+}\left(x^{\prime}\right)\right\rangle\left\langle\psi_{-}(x), \psi_{-}\left(x^{\prime}\right)\right\rangle
\end{array}\right) & \\
= & \left(\begin{array}{l}
{\left[\psi_{+}(x), \psi_{+}\left(x^{\prime}\right)\right]_{+}\left[\psi_{+}(x), \psi_{-}\left(x^{\prime}\right)\right]_{+}} \\
{\left[\psi_{-}(x), \psi_{+}\left(x^{\prime}\right)\right]_{+}\left[\psi_{-}(x), \psi_{-}\left(x^{\prime}\right)\right]_{+}}
\end{array}\right) \\
= & \frac{1}{m}\left(\begin{array}{cc}
\partial_{0}-\partial_{1} & m+\partial_{2} \\
-m+\partial_{2} & \partial_{0}+\partial_{1}
\end{array}\right) \Delta\left(x-x^{\prime} ; m^{2}\right) .
\end{aligned}
$$

The representation of the Poincare group in $W_{F}$ is given by

$$
\left(\begin{array}{l}
\left(\varpi_{F}(\tilde{g}) w\right)_{+}(x) \\
\left(\varpi_{F}(\widetilde{g}) w\right)_{-}(x)
\end{array}\right)=S(g)\left(\begin{array}{l}
w_{+}\left(\widetilde{g}^{-1} x\right) \\
w_{-}\left(\widetilde{g}^{-1} x\right)
\end{array}\right) .
$$

Here $S$ is the spinor representation of $S O_{0}(1,2)$ whose infinitesimal form is given by

$$
d S\left(M^{\mu}\right)=S^{\mu}
$$

where

$$
\begin{aligned}
& M^{0}=\left(\begin{array}{rrr}
0 & 0 & 0 \\
0 & 0 & 1 \\
0 & -1 & 0
\end{array}\right), \quad M^{1}=\left(\begin{array}{lll}
0 & 0 & 1 \\
0 & 0 & 0 \\
1 & 0 & 0
\end{array}\right), \quad M^{2}=\left(\begin{array}{lll}
0 & 1 & 0 \\
1 & 0 & 0 \\
0 & 0 & 0
\end{array}\right) \\
& S^{0}=\frac{1}{2}\left(\begin{array}{l}
-1 \\
1
\end{array}\right), \quad S^{1}=\frac{-1}{2}\left(\begin{array}{l}
1 \\
1
\end{array}\right), \quad S^{2}=\frac{1}{2}\left(\begin{array}{r}
1 \\
-1
\end{array}\right)
\end{aligned}
$$


Now applying (A. 18) $)_{F}$ and (A. 19) ${ }_{F}$ in the Appendix we shall compute $\operatorname{Nr}\left(\varphi_{F}\left(a^{*}\right)\right)$. Similarly to (4.1.37) $\varphi_{F}\left(a^{*}\right)$ is to satisfy the following commutation with $\psi_{ \pm}(x)$.

(4.2.31) $\varphi_{F}\left(a^{*}\right) \psi(x)$

$$
=\left\{\begin{aligned}
\psi(x) \varphi_{F}\left(a^{*}\right) & \text { if } x \cdot \xi_{+}-r_{+}>0 \text { and } x \cdot \xi_{-}-r_{-}<0 \\
-\psi(x) \psi_{F}\left(a^{*}\right) & \text { if } x \cdot \xi_{+}-r_{+}<0 \text { and } x \cdot \xi_{-}-r_{-}>0 .
\end{aligned}\right.
$$

First we assume that $a^{*}$ is given by (4.1.32), and denote it by $a_{0}^{*}$.

We set

$$
\widehat{\psi}_{ \pm}(\vec{p})=\int d^{2} \vec{x} \psi_{ \pm}(0, \vec{x}) e^{-i \vec{x} \cdot \vec{p}} .
$$

In what follows we identify a kernel function $F\left(\vec{p}, \vec{p}^{\prime}\right)$ $=\left(\begin{array}{ll}F_{1}\left(\vec{p}, \vec{p}^{\prime}\right) & F_{2}\left(\vec{p}, \vec{p}^{\prime}\right) \\ F_{3}\left(\vec{p}, \vec{p}^{\prime}\right) & F_{4}\left(\vec{p}, \vec{p}^{\prime}\right)\end{array}\right)$ with the linear transformation $F$ such that

$$
\left(F \widehat{\psi}_{+}\left(\vec{p}^{\prime}\right), F \widehat{\psi}_{-}\left(\vec{p}^{\prime}\right)\right)=\int \frac{d^{2} \vec{p}}{(2 \pi)^{2}}\left(\widehat{\psi}_{+}(\vec{p}), \widehat{\psi}_{-}(\vec{p})\right) F\left(\vec{p}, \vec{p}^{\prime}\right) .
$$

Then we have

$$
\begin{aligned}
& K\left(\vec{p}, \vec{p}^{\prime}\right) \underset{\text { def }}{=}\left(\begin{array}{ll}
\left\langle\hat{\psi}_{+}(\vec{p}) \hat{\psi}_{+}\left(\vec{p}^{\prime}\right)\right\rangle\left\langle\widehat{\psi}_{+}(\vec{p}) \widehat{\psi}_{-}\left(\vec{p}^{\prime}\right)\right\rangle \\
\left\langle\hat{\psi}_{-}(\vec{p}) \widehat{\psi}_{+}\left(\vec{p}^{\prime}\right)\right\rangle\left\langle\widehat{\psi}_{-}(\vec{p}) \widehat{\psi}_{-}\left(\vec{p}^{\prime}\right)\right\rangle
\end{array}\right) \\
& =\frac{1}{m \omega(\vec{p})}\left(\begin{array}{cc}
\omega(\vec{p})+p_{1} & i\left(m+i p_{2}\right) \\
-i\left(m-i p_{2}\right) & \omega(\vec{p})-p_{1}
\end{array}\right)(2 \pi)^{2} \delta\left(\vec{p}+\vec{p}^{\prime}\right),
\end{aligned}
$$

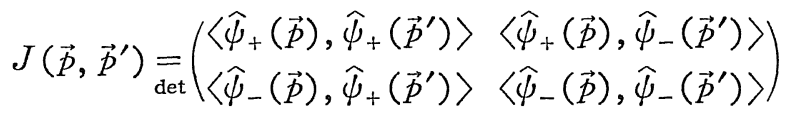

$$
\begin{aligned}
& =\frac{2}{m}(2 \pi)^{2} \delta(\vec{p}+\vec{p}) \cdot I_{2},
\end{aligned}
$$

(4.2.35) $\quad E\left(\vec{p}, \vec{p}^{\prime}\right)=\frac{1}{\omega(\vec{p})}\left(\begin{array}{cc}-p_{1} & i\left(m-i p_{2}\right) \\ -i\left(m+i p_{2}\right) & p_{1}\end{array}\right)(2 \pi)^{2} \delta\left(\vec{p}-\vec{p}^{\prime}\right)$

In this case $X_{ \pm}$in $(\mathrm{A} .18)^{\prime}$ are given by

(4.2.36) $\quad X_{+}\left(\vec{p}, \vec{p}^{\prime}\right)$

$=\left(\begin{array}{c}\sqrt{p_{1}+i \mu(\vec{p})^{-1}} \\ \sqrt{p_{1}+i \mu(\vec{p})}\end{array}\right) \frac{1}{\sqrt{2}}\left(\begin{array}{rr}1 & 1 \\ -1 & 1\end{array}\right) \frac{1}{\sqrt{\mu(\vec{p})}}\left(\begin{array}{c}\sqrt{m+i p_{2}} \\ \sqrt{m-i p_{2}}\end{array}\right)(2 \pi)^{2} \delta\left(\vec{p}-\vec{p}^{\prime}\right)$, 
(4.2.37) $X_{-}\left(\vec{p}, \vec{p}^{\prime}\right)$

$=\left(\begin{array}{c}\sqrt{p_{1}-i \mu(\vec{p})^{-1}} \\ \sqrt{p_{1}-i \mu(\vec{p})}\end{array}\right) \frac{1}{\sqrt{2}}\left(\begin{array}{rr}1 & 1 \\ -1 & 1\end{array}\right) \frac{1}{\sqrt{\mu(\vec{p})}}\left(\begin{array}{c}\sqrt{m+i p_{2}} \\ \sqrt{m-i p_{2}}\end{array}\right)(2 \pi)^{2} \delta\left(\vec{p}-\vec{p}^{\prime}\right)$.

Nothing that

$$
\frac{\sqrt{\omega(\vec{p})+p_{1}} \pm i \sqrt{\omega(\vec{p})-p_{1}}}{\sqrt{2}}=\sqrt{p_{1} \pm i \mu(\vec{p})}
$$

we have from (4.1.45), (4.2.23), (4.2.34) (4.2.36) and (A. 19) ${ }_{F}$ (4. 2. 39) $\quad \operatorname{Nr}\left(\varphi_{F}\left(a_{0}^{*}\right)\right)=\exp \left(\rho_{F}\left(a_{0}^{*}\right) / 2\right)$

where $\quad \rho_{F}\left(a_{0}^{*}\right)=\iint \frac{d p}{d p^{\prime}} \frac{-i\left(p_{0}-p_{0}^{\prime}\right)}{p_{1}+p_{1}^{\prime}-i 0} \cdot 2 \pi \delta\left(p_{2}+p_{2}^{\prime}\right) \psi(p) \psi\left(p^{\prime}\right)$.

Let $\left(\xi_{+}, \xi_{-}\right)$be an ordered pair of positively lightlike vectors in $P$. We choose an element $g$ in $S O_{0}(1,2)$ which satisfies $g\left(\begin{array}{r}1 \\ \mp 1 \\ 0\end{array}\right)=c \xi_{ \pm}$for some positive constant $c$. We set $\xi^{\perp}=g\left(\begin{array}{l}0 \\ 0 \\ 1\end{array}\right)$. We denote by $\psi^{\prime}(p)$ $=\left(\begin{array}{l}v_{+}^{\prime}(x ; p) \\ v_{-}^{\prime}(x ; p)\end{array}\right)$ the solution of (4.2.1) given by

$$
\left(\begin{array}{l}
v_{+}^{\prime}(x ; p) \\
v_{-}^{\prime}(x ; p)
\end{array}\right)=S(g)\left(\frac{\sqrt{m^{2}+\left(p \cdot \xi^{\perp}\right)^{2}}}{m}\right)^{1 / 2}\left(\begin{array}{c}
\sqrt{\frac{0-i p \cdot \xi_{+}}{m-i p \cdot \xi^{\perp}}} \\
\sqrt{\frac{0+i p \cdot \xi_{-}}{m+i p \cdot \xi^{\perp}}}
\end{array}\right) e^{i x \cdot p} .
$$

$\psi^{\prime}(p)$ is independent of the choice of $g$. It is uniquely determined by the pair of lightlike directions $\left(\left\{c \xi_{+} \mid c>0\right\},\left\{c \xi_{-} \mid c>0\right\}\right)$.

Theorem 4. 2. 1. Normalizing $\varphi_{F}\left(a^{*}\right)$ so that $\left\langle\varphi_{F}\left(a^{*}\right)\right\rangle=1$, we have

$$
\operatorname{Nr}\left(\varphi_{F}\left(a^{*}\right)\right)=\exp \left(\rho_{F}\left(a^{*}\right) / 2\right)
$$

where

$$
\begin{aligned}
\rho_{F}\left(a^{*}\right)= & \iint \underline{d p} \frac{d p^{\prime}}{\left(p+p^{\prime}\right) \cdot \xi_{+}-i 0} \cdot 2 \pi \delta\left(\left(p+p^{\prime}\right) \cdot \xi^{\perp}\right) \\
& \times e^{-i x\left(a^{*}\right)\left(p+p^{\prime}\right)} \phi^{\prime}(p) \phi^{\prime}\left(p^{\prime}\right) .
\end{aligned}
$$


Let us now proceed to the case of 2-dimensional space-time.

The 2-dimensional Dirac equation reads

$$
\left(\begin{array}{cc}
m & \partial_{0}-\partial_{1} \\
-\partial_{0}-\partial_{1} & m
\end{array}\right)\left(\begin{array}{l}
w_{+}(x) \\
w_{-}(x)
\end{array}\right)=0,
$$

and for solutions $w=\left(\begin{array}{c}w_{+}(x) \\ w_{-}(x)\end{array}\right), w^{\prime}=\left(\begin{array}{c}w_{+}^{\prime}(x) \\ w_{-}^{\prime}(x)\end{array}\right)$ of $(4.2 .42)$,

$$
\begin{aligned}
\mathcal{J}_{F}\left(w, w^{\prime}\right)= & \left(w_{+}(x) w_{+}^{\prime}(x)+w_{-}(x) w_{-}^{\prime}(x)\right) d x_{1} \\
& -\left(w_{+}(x) w_{+}^{\prime}(x)-w_{-}(x) w_{-}^{\prime}(x)\right) d x_{0}
\end{aligned}
$$

is a closed 1-form. Starting from (4.2.42) and (4.2.43), we may construct an analogous theory as in the 3-dimensional case. The results are exactly the same as the 3 -dimensional case if we set the $p_{2}$-coordinate to be zero.

Using the coordinates (4.1.60) and (4.1.61), we write $\psi(u)$ in place of $\psi(p)$. Then we have

$$
\left\langle\psi(u) \psi\left(u^{\prime}\right)\right\rangle=2 \pi u u_{+} \delta\left(u+u^{\prime}\right),
$$

$$
\left\langle\psi(u), \psi\left(u^{\prime}\right)\right\rangle=2 \pi|u| \delta\left(u+u^{\prime}\right) .
$$

(4.2.23) and (4.2.41) reduce to

$$
\psi_{ \pm}(x)=\int d u \sqrt{0+i u}^{ \pm 1} \psi(u) e^{-i m\left(x^{-} u+x^{+} u-1\right)},
$$

$$
\operatorname{Nr}\left(\varphi_{F}(a)\right)=\exp \left(\rho_{F}(a) / 2\right)
$$

where $\rho_{F}(a)=\iint \frac{d u}{d u^{\prime}} \frac{-i\left(u-u^{\prime}\right)}{u+u^{\prime}-i 0} e^{-i m\left(a-\left(u+u^{\prime}\right)+a^{+}\left(u-1+u^{\prime-1}\right)\right)} \phi(u) \phi\left(u^{\prime}\right)$.

\section{§ 4. 3. Local Operator Expansions}

In this and subsequent sections we restrict ourselves to the case $\operatorname{dim} X^{\mathrm{Min}}=2$.

We have constructed operators $\varphi_{B}(a)$ or $\varphi_{F}(a)$ which satisfies the commutation relation (4.1.18) or (4.2.16) respectively. In this section we shall construct similar operators which satisfy more general commutation relations, and then we shall derive local operator expansions.

First we treat the symplectic case. We consider $\mathbb{C}^{2}=\mathbb{C} e \oplus \mathbb{C} e^{*}$ to be an orthogonal vector space with the inner product $\langle,\rangle_{\boldsymbol{C}^{2}}$ given by 


$$
\left(\begin{array}{cc}
\langle e, e\rangle_{\boldsymbol{C}^{2}} & \left\langle e, e^{*}\right\rangle_{\boldsymbol{C}^{2}} \\
\left\langle e^{*}, e\right\rangle_{\boldsymbol{C}^{2}} & \left\langle e^{*}, e^{*}\right\rangle_{\boldsymbol{C}^{2}}
\end{array}\right)=\left(\begin{array}{ll} 
& 1 \\
1 &
\end{array}\right) .
$$

We shall construct the operator theory starting from the symplectic vector space $W_{B} \otimes C^{2}$, where the inner product is given by

$$
\left(\begin{array}{cc}
\left\langle v \otimes e, v^{\prime} \otimes e\right\rangle & \left\langle v \otimes e, v^{\prime} \otimes e^{*}\right\rangle \\
\left\langle v \otimes e^{*}, v^{\prime} \otimes e\right\rangle & \left\langle v \otimes e^{*}, v^{\prime} \otimes e^{*}\right\rangle
\end{array}\right)=\left(\begin{array}{ll}
\left\langle v, v^{\prime}\right\rangle \\
\left\langle v, v^{\prime}\right\rangle &
\end{array}\right) .
$$

In what follows $\phi(u) \otimes e$ and $\phi(u) \otimes e^{*}$ are denoted simply by $\phi(u)$ and $\phi^{*}(u)$ respectively. They satisfy the following.

$$
\begin{aligned}
& \left(\begin{array}{cc}
\left\langle\phi(u), \phi\left(u^{\prime}\right)\right\rangle & \left\langle\phi(u), \phi^{*}\left(u^{\prime}\right)\right\rangle \\
\left\langle\phi^{*}(u), \phi\left(u^{\prime}\right)\right\rangle & \left\langle\phi^{*}\langle u\rangle, \phi^{*}\left(u^{\prime}\right)\right\rangle
\end{array}\right) \\
& =\left(\begin{array}{cc}
{\left[\phi(u), \phi\left(u^{\prime}\right)\right]} & {\left[\phi(u), \phi^{*}\left(u^{\prime}\right)\right]} \\
{\left[\phi^{*}(u), \phi\left(u^{\prime}\right)\right]} & {\left[\phi^{*}(u), \phi^{*}\left(u^{\prime}\right)\right]}
\end{array}\right) \\
& =\left(\begin{array}{cc}
0 & 2 \pi u \delta\left(u+u^{\prime}\right) \\
2 \pi u \delta\left(u+u^{\prime}\right) & 0
\end{array}\right), \\
& \left(\begin{array}{ccc}
\left\langle\phi(u) \phi\left(u^{\prime}\right)\right\rangle & \left\langle\phi(u) \phi^{*}\left(u^{\prime}\right)\right\rangle \\
\left\langle\phi^{*}(u) \phi\left(u^{\prime}\right)\right\rangle & \left\langle\phi^{*}(u) \phi^{*}\left(u^{\prime}\right)\right\rangle
\end{array}\right) \\
& =\left(\begin{array}{cc}
0 & 2 \pi u_{+} \delta\left(u+u^{\prime}\right) \\
2 \pi u+\delta\left(u+u^{\prime}\right) & 0
\end{array}\right) .
\end{aligned}
$$

We set for $l \in \boldsymbol{C}$

$$
\begin{aligned}
& \left.\phi_{l}(x)=\int \underline{d u}(0+i u)^{l} e^{-i m\left(x^{-u}+x^{+} u-1\right.}\right) \phi(u), \\
& \phi_{l}^{*}(x)=\int \underline{d u}(0+i u)^{l} e^{-i m\left(x^{-u}+x^{+}+u^{-1}\right)} \phi^{*}(u),
\end{aligned}
$$

where $(0+i u)^{l}=e^{\pi i l / 2}(u-i 0)^{l}=e^{ \pm \pi i l / 2}|u|^{l}$ if $u \gtrless 0$.

From the formulas

$$
\begin{aligned}
& \int_{0}^{\infty} \frac{d u}{u^{l} e^{ \pm i m\left(x-u+x^{+} u^{-1}\right)}} \\
& =\frac{e^{(1 \mp 1 / 2) \pi i l}}{\pi} \widetilde{v}_{-l}\left(-x^{-} \mp i 0, x^{+} \pm i 0\right)^{(*)}
\end{aligned}
$$

(*) For the definitions of $v_{l}, v_{l}^{*}$ and $\widetilde{v}_{l}$ see Section 3.1 


$$
\begin{gathered}
=\frac{c^{\mp(\pi / 2) i l}}{2 \sin \pi l}\left(v_{-l}\left(-x^{-} \mp i 0, x^{+} \pm i 0\right)-v_{l}^{*}\left(-x^{-} \mp i 0, x^{+} \pm i 0\right)\right) \\
(\text { for } l \notin \mathbb{Z}),
\end{gathered}
$$

we have

$$
\begin{aligned}
& \text { (4.3.8) }\left\langle\phi_{l}(x) \phi_{l^{\prime}}^{*}\left(x^{\prime}\right)\right\rangle=\left\langle\phi_{l}^{*}(x) \phi_{l^{\prime}}\left(x^{\prime}\right)\right\rangle \\
& ={ }^{2 \pi i l} e^{\pi i l^{\prime}} \widetilde{v}_{-\left(l+l^{\prime}\right)}\left(-\left(x-x^{\prime}\right)^{-}+i 0,\left(x-x^{\prime}\right)^{+}-i 0\right) \\
& =\frac{e^{\pi i l}}{2 \sin \pi\left(l+l^{\prime}\right)}\left(v_{-\left(l+l^{\prime}\right)}\left(-\left(x-x^{\prime}\right)^{-}+i 0,\left(x-x^{\prime}\right)^{+}-i 0\right)\right. \\
& \left.-v_{l+l^{\prime}}^{*}\left(-\left(x-x^{\prime}\right)^{-}+i 0,\left(x-x^{\prime}\right)^{+}-i 0\right)\right) \quad\left(\text { for } l+l^{\prime} \notin \mathbb{Z}\right) .
\end{aligned}
$$

From (4.1.26) we see that $\phi_{0}(x)$ (resp. $\phi_{0}^{*}(x)$ ) is nothing but $\phi(x)$ $\otimes e$ (resp. $\left.\phi(x) \otimes e^{*}\right)$, which we denote simply by $\phi(x) \quad\left(\right.$ resp. $\left.\phi^{*}(x)\right)$. We define the norm $\rho_{B}(a ; l)$ by

$$
\begin{aligned}
\rho_{B}(a ; l)= & 2 \iint d u d u^{\prime} R_{B}\left(u, u^{\prime} ; l\right) \phi(u) \phi^{*}\left(u^{\prime}\right) \\
& \times e^{-i m\left(a-\left(u+u^{\prime}\right)+a^{+}\left(u^{-1}+u^{\prime-1}\right)\right)},
\end{aligned}
$$

where

$$
R_{B}\left(u, u^{\prime} ; l\right)=-2 \sin \pi l\left(\begin{array}{c}
u-i 0 \\
u^{\prime}-i 0
\end{array}\right)^{-\imath+(1 / 2) \sqrt{u-i 0} \sqrt{ } u^{\prime}-i 0} \frac{u+u^{\prime}-i 0}{u}
$$

and define operators $\varphi_{B}(a ; l), \varphi_{l^{\prime}}^{B}(a ; l)$ and $\varphi_{l^{\prime}}^{B^{\prime}}(a ; l)$ by

$$
\operatorname{Nr}\left(\varphi_{B}(a ; l)\right)=\exp \left(\rho_{B}(a ; l) / 2\right),
$$

$$
\operatorname{Nr}\left(\varphi_{l^{\prime}}^{B}(a ; l)\right)=\phi_{l^{\prime}}(a) \exp \left(\rho_{B}(a ; l) / 2\right),
$$

$$
\operatorname{Nr}\left(\varphi_{l^{\prime}}^{B^{*}}(a ; l)\right)=\phi_{l^{\prime}}^{*}(a) \exp \left(\rho_{B}(a ; l) / 2\right) .
$$

Note that

$$
R_{B}\left(u, u^{\prime} ; l\right)=R_{B}\left(u^{\prime}, u ; 1-l\right),
$$

and that for $l=\frac{1}{2}$ (4.3.10) reduces to the kernel in (4.1.65).

Now we assume that $l \notin Z$. Applying the formula (A. 10) (A. 11) we have the following.

$$
\operatorname{Nr}\left(\phi(x) \varphi_{B}(a ; l)\right)=\phi^{(1)} \operatorname{Nr}\left(\varphi_{B}(a ; l)\right)
$$




$$
\begin{aligned}
& \phi^{(1)}=\int \underline{d u \xi_{l}^{(1)}}(x-a ; u) e^{-i m\left(a-u+a^{+} u^{-1}\right)} \phi(u), \\
& \xi_{l}^{(1)}(x ; u)=e^{-i m\left(x^{-} u+x^{+} u^{-1}\right)} \\
& \quad+\int_{-\infty}^{0} \frac{d u^{\prime}}{} R_{B}\left(u, u^{\prime} ; l\right) e^{i m\left(x^{-} u^{\prime}+x^{+} u^{\prime}-1\right)}
\end{aligned}
$$

(4. 3. 16) $\operatorname{Nr}\left(\phi^{*}(x) \varphi_{B}(a ; l)\right)=\phi^{(1) *} \operatorname{Nr}\left(\varphi_{B}(a ; l)\right)$,

$$
\phi^{(1) *}=\int d u \xi_{1-\imath}^{(1)}(x-a ; u) e^{-i m\left(a-u+a^{+} u^{-1}\right)} \phi^{*}(u) .
$$

(4. 3. 17) $\operatorname{Nr}\left(\varphi_{B}(a ; l) \phi(x)\right)=\phi^{(2)} \operatorname{Nr}\left(\varphi_{B}(a ; l)\right)$,

$$
\begin{aligned}
& \phi^{(2)}=\int \underline{d u} \xi_{l}^{(2)}(x-a ; u) e^{-i m\left(a-u+a^{+} u^{-1}\right)} \phi(u) \\
& \xi_{i}^{(2)}(x ; u)= e^{-i m\left(x^{-} u+x^{+} u^{-1}\right)} \\
&+\int_{0}^{\infty} d u^{\prime} R_{B}\left(u, u^{\prime} ; l\right) e^{i m\left(x^{-} u^{\prime}+x^{+} u^{\prime}-1\right)}
\end{aligned}
$$

(4. 3. 18) $\operatorname{Nr}\left(\varphi_{B}(a ; l) \phi^{*}(x)\right)=\phi^{(2) *} \operatorname{Nr}\left(\varphi_{B}(a ; l)\right)$,

$$
\phi^{(2) *}=\int d u \xi_{1-l}^{(2)}(x-a ; u) e^{-i m\left(a-u+a^{+} u-1\right)} \phi^{*}(u) .
$$

Proposition 4. 3. 1. We assume that $l \notin Z$. We set

(4.3.19) $\quad \xi_{l}(x ; u)=\sum_{j=0}^{\infty}(0+i u)^{-i+1+j} v_{-l+1+j}\left(-x^{-}, x^{+}\right)$

$$
+\sum_{j=0}^{\infty}(0+i u)^{-l-j} v_{l+j}^{*}\left(-x^{-}, x^{+}\right) .
$$

$\xi_{l}(x ; u)$ satisfies

(4. 3. 20) $\quad\left(m^{-1} \partial_{x^{-}}+i u\right) \xi_{l}(x ; u)$

$$
=-(0+i u)^{-l+1}\left(v_{-l}\left(-x^{-}, x^{+}\right)-v_{l}^{*}\left(-x^{-}, x^{+}\right)\right),
$$

(4. 3. 21) $\quad\left(m^{-1} \partial_{x^{+}}+i u^{-1}\right) \xi_{l}(x ; u)$

$$
=-(0+i u)^{-i}\left(v_{-l+1}\left(-x^{-}, x^{+}\right)-v_{l-1}^{*}\left(-x^{-}, x^{+}\right)\right) \text {. }
$$

$\xi_{l}^{(1)}(x ; u)$ and $\xi_{l}^{(2)}(x ; u)$ are respectively given by the following boundary values of the multi-valued analytic function $\xi_{l}(x ; u)$.

(4. 3. 22) $\xi_{l}^{(1)}(x ; u)=\xi_{l}\left(-x^{-}+i 0, x^{+}-i 0\right)$, 
(4. 3. 23) $\quad \xi_{l}^{(2)}(x ; u)=\xi_{l}\left(-x^{-}-i 0, x^{+}+i 0\right)$.

In particular, we have

(4. 3. 24) $\xi_{l}^{(1)}(x ; u)=\xi_{l}^{(2)}(x ; u) \quad$ if $x^{+}>0$ and $x^{-}<0$,

(4. 3. 25) $\xi_{l}^{(1)}(x ; u)=e^{-2 \pi i l} \xi_{l}^{(2)}(x ; u) \quad$ if $x^{+}<0$ and $x^{-}>0$.

Proof. If $u^{\prime}<0$ and $\operatorname{Im} x^{=}<0$, Re $i m\left(x^{-} u^{\prime}+x^{+} u^{\prime-1}\right)<0$. Hence $\xi_{l}^{(1)}(x ; u)$ is the boundary value of a holomorphic function in the region $\left\{x \in \mathbb{C}^{2} \mid \operatorname{Im} x^{ \pm}<0\right\}$. Likewise $\xi_{l}^{(2)}(x ; u)$ is a boundary value from $\left\{x \in \mathbb{C}^{2} \mid\right.$ $\left.\operatorname{Im} x^{ \pm}>0\right\}$.

We have

$$
\begin{aligned}
\xi_{l}^{(1)}(x ; u)-\xi_{l}^{(2)}(x ; u)= & \int_{-\infty}^{\infty} \frac{d u^{\prime}}{2 \pi} 2 \sin \pi l \frac{(u-i 0)^{-l+1}\left(u^{\prime}-i 0\right)^{l-1}}{u+u^{\prime}-i 0} \\
& \times e^{i m\left(x-u^{\prime}+x^{\prime} u^{\prime-1}\right)} .
\end{aligned}
$$

If $x^{+}>0$ and $x^{-}<0$, the contour may be closed in the lower half plane to yield 0. Hence (4.3.24) is valid. Similarly we have (4.3.25). (4. 3. 24) implies that $\xi_{l}^{(1)}(x ; u)$ and $\xi_{l}^{(2)}(x ; u)$ are boundary values of the same analytic function, which we denote by $\xi_{l}^{\prime}(x ; u)$.

It is easy to check that both $\xi_{l}(x ; \imath)$ and $\xi_{l}^{\prime}(x ; \imath)$ satisfy (4.3.20) and (4.3.21). (See (4.3.7).) Hence these two differ only by $c(u) e^{-i m\left(x^{-} u+x^{+} u^{-1}\right)}$. Since they satisfy the monodromy property (4.3.24) and (4.3.25), we conclude that $c(u)=0$.

Theorem 4. 3.2. Assuming that $l \notin \mathbb{Z}$, we have (4. 3. 26) $\phi(x) \varphi_{B}(a ; l)$

$$
\begin{gathered}
=\sum_{j=0}^{\infty} \varphi_{-l+1+j}^{B}(a ; l) v_{-l+1+j}\left(-(x-a)^{-}+i 0,(x-a)^{+}-i 0\right) \\
+\sum_{j=0}^{\infty} \varphi_{-l-j}^{B}(a ; l) v_{l+j}^{*}\left(-(x-a)^{-}+i 0,(x-a)^{+}-i 0\right),
\end{gathered}
$$

(4.3.27) $\phi^{*}(x) \varphi_{B}(a ; l)$

$$
\begin{aligned}
= & \sum_{j=0}^{\infty} \varphi_{l+j}^{B *}(a ; l) v_{l+j}\left(-(x-a)^{-}+i 0,(x-a)^{+}-i 0\right) \\
& +\sum_{j=0}^{\infty} \varphi_{l-1-j}^{B *}(a ; l) v_{-l+1+j}^{*}\left(-(x-a)^{-}+i 0,(x-a)^{+}-i 0\right) .
\end{aligned}
$$


$\varphi_{B}(a ; l) \phi(x) \quad\left(\right.$ resp. $\left.\varphi_{B}(a ; l) \phi^{*}(x)\right)$ is given by the right hand side of (4.3.26) (resp. (4.3.27)) with $\mp(x-a)^{\mp} \pm i 0$ replaced by $\mp(x$ $-a)^{\mp} \mp i 0$.

In particular, we have the following commutation relations in the region where $x$ and a are mutually spacelike.

(4. 3. 28) $\varphi_{B}(a ; l) \phi(x)$

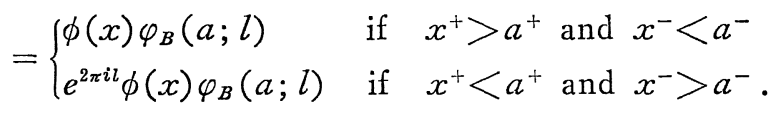

(4.3.29) $\varphi_{B}(a ; l) \phi^{*}(x)$

$$
=\left\{\begin{array}{lll}
\phi^{*}(x) \varphi_{B}(a ; l) & \text { if } & x^{+}>a^{+} \text {and } x^{-}<a^{-} \\
e^{-2 \pi i l} \phi^{*}(x) \varphi_{B}(a ; l) & \text { if } & x^{+}<a^{+} \text {and } x^{-}>a^{-} .
\end{array}\right.
$$

Proof. These are the direct consequences of (A. 8) and Proposition 4. 3. 1 .

We define furthermore the following operators.

$$
\begin{aligned}
& \operatorname{Nr}\left(\varphi_{B l_{1}, l_{2}}(a ; l)\right)=\phi_{l_{1}}(a) \phi_{l_{2}}(a) e^{\rho_{B}(a ; l) / 2} . \\
& \operatorname{Nr}\left(\varphi_{B l_{1}, l_{2}}^{*}(a ; l)\right)=\phi_{l_{1}}^{*}(a) \phi_{l_{2}}^{*}(a) c^{\rho_{B}(a ; l) / 2} . \\
& \operatorname{Nr}\left(\varphi_{B l_{1}, l_{2}}^{*}(a ; l)\right)=\operatorname{Nr}\left(\varphi_{B l_{2}, l_{1}}^{*}(a ; l)\right) \\
& \quad=\phi_{l_{1}}(a) \phi_{l_{2}}^{*}(a) e^{\rho_{B}(a ; l) / 2}=\phi_{l_{2}}^{*}(a) \phi_{l_{1}}(a) e^{\rho_{B}(a ; l) / 2} .
\end{aligned}
$$

Then from (A. 10), (4.3.8) and Theorem 4.3.2 we have the following theorem.

Theorem 4. 3. 3. Assuming $l, l^{\prime} \notin Z$, we have

$$
\begin{aligned}
\phi(x) & \varphi_{l^{\prime}}^{B}(a ; l) \\
= & \sum_{j=0}^{\infty} \varphi_{B}-l+1+j, l^{\prime} \\
& (a ; l) v_{-l+1+j}\left(-(x-a)^{-}+i 0,(x-a)^{+}-i 0\right) \\
& +\sum_{j=0}^{\infty} \varphi_{B-l-j, l^{\prime}}(a ; l) v_{l+j}^{*}\left(-(x-a)^{-}+i 0,(x+a)^{+}-i 0\right),
\end{aligned}
$$

(4. 3. 34) $\phi(x) \varphi_{l}^{B *}(a ; l)$

$$
=\frac{1}{2 \sin \pi l^{\prime}} \varphi_{B}(a ; l) v_{-l^{\prime}}\left(-(x-a)^{-}+i 0,(x-a)^{+}-i 0\right)
$$




$$
\begin{aligned}
& +\sum_{j=0}^{\infty} \varphi_{B l^{\prime},-l+1+j}^{*}(a ; l) v_{-l+1+j}\left(-(x-a)^{-}+i 0,(x-a)^{+}-i 0\right) \\
& -\frac{1}{2 \sin \pi l^{\prime}} \varphi_{B}(a ; l) v_{l^{\prime}}^{*}\left(-(x-a)^{-}+i 0,(x-a)^{+}-i 0\right) \\
& +\sum_{j=0}^{\infty} \varphi_{B l^{\prime},-l-j}^{*}(a ; l) v_{l+j}^{*}\left(-(x-a)^{-}+i 0,(x-a)^{+}-i 0\right),
\end{aligned}
$$

(4. 3. 35) $\phi^{*}(x) \varphi_{l^{\prime}}^{B *}(a ; l)$

$$
\begin{aligned}
= & \sum_{j=0}^{\infty} \varphi_{B}{ }_{l+j}^{*} l^{\prime}(a ; l) v_{l+j}\left(-(x-a)^{-}+i 0,(x-a)^{+}-i 0\right) \\
& +\sum_{j=0}^{\infty} \varphi_{B} l_{l-1-j}^{*} i^{\prime}(a ; l) v_{-l+1+j}^{*}\left(-(x-a)^{-}+i 0,(x-a)^{+}-i 0\right),
\end{aligned}
$$

(4.3.36) $\phi^{*}(x) \varphi_{l^{\prime}}^{B}(a ; l)$

$$
\begin{aligned}
= & \frac{1}{2 \sin \pi l^{\prime}} \varphi_{B}(a ; l) v_{-l^{\prime}}\left(-(x-a)^{-}+i 0,(x-a)^{+}-i 0\right) \\
& +\sum_{j=0}^{\infty} \varphi_{B l^{\prime}, l^{* j}}(a ; l) v_{l+j}\left(-(x-a)^{-}+i 0,(x-a)^{+}-i 0\right) \\
& -\frac{1}{2 \sin \pi l^{\prime}} \varphi_{B}(a ; l) v_{l^{\prime}}^{*}\left(-(x-a)^{-}+i 0,(x-a)^{+}-i 0\right) \\
& +\sum_{j=0}^{\infty} \varphi_{B l^{\prime}, i^{*}-j-j}(a ; l) v_{-l+1+j}^{*}\left(-(x-a)^{-}+i 0,(x-a)^{+}-i 0\right) .
\end{aligned}
$$

$\varphi_{l^{\prime}}^{B}(a ; l) \phi(x), \varphi_{l^{\prime}}^{B^{*}}(a ; l) \phi(x)$, etc. are given by the right hand sides of $(4.3 .33),(4.3 .34)$, etc. with $\mp(x-a)^{\mp} \pm i 0$ replaced by $\mp(x-a)^{\mp}$ $\mp i 0$.

In particular, if $l^{\prime} \equiv-l(\bmod \mathbb{Z})$ we have

(4.3.37) $\varphi_{l^{\prime}}^{B}(a ; l) \phi(x)$

$$
\begin{aligned}
& =\left\{\begin{array}{lll}
\phi(x) \varphi_{l^{*}}^{B *}(a ; l) & \text { if } & x^{+}>a^{+} \text {and } x^{-}<a^{-} \\
e^{2 \pi i l} \phi(x) \varphi_{l^{*}} *(a ; l) & \text { if } & x^{+}<a^{+} \text {and } x^{-}>a^{-} .
\end{array}\right. \\
\varphi_{l^{\prime}}^{B}(a ; l) \phi^{*}(x) & \\
& =\left\{\begin{array}{lll}
\phi^{*}(x) \varphi_{l^{\prime}}^{B}(a ; l) & \text { if } & x^{+}>a^{+} \text {and } x^{-}<a^{-} \\
e^{-2 \pi i l} \phi^{*}(x) \varphi_{l^{\prime}}^{B}(a ; l) & \text { if } & x^{+}<a^{+} \text {and } x^{-}>a^{-},
\end{array}\right.
\end{aligned}
$$

and if $l^{\prime} \equiv l(\bmod Z \mathbf{Z})$ we have

(4.3.38) $\varphi_{l^{\prime}}^{B^{*}}(a ; l) \phi(x)$ 


$$
\begin{gathered}
=\left\{\begin{array}{lll}
\phi(x) \varphi_{l^{\prime}}^{B^{*}}(a ; l) & \text { if } & x^{+}>a^{+} \text {and } x^{-}<a^{-} \\
e^{2 \pi i l} \phi(x) \varphi_{l^{\prime}}^{B^{+}}(a ; l) & \text { if } & x^{+}<a^{+} \text {and } x^{-}>a^{-},
\end{array}\right. \\
\varphi_{l^{\prime}}^{B^{\prime}}(a ; l) \phi^{*}(x) \\
=\left\{\begin{array}{lll}
\phi^{*}(x) \varphi_{l^{\prime}}^{B^{\prime}}(a ; l) & \text { if } & x^{+}>a^{+} \text {and } x^{-}<a^{-} \\
c^{-2 \pi i l} \phi^{*}(x) \varphi_{l^{\prime}}^{B^{\prime}}(a ; l) & \text { if } & x^{+}<a^{+} \text {and } x^{-}>a^{-} .
\end{array}\right.
\end{gathered}
$$

Remark. (4.3.28) and (4.3.29) imply that $\varphi_{B}(a ; l)$ induces the symplectic rotation in $W_{B} \otimes \mathbb{C}^{2}$. Since $\varphi_{-l+j}^{B}(a ; l)(j \in \mathbb{Z})$ appears as a coefficient of the local expansion of $\phi(x) \varphi_{B}(a ; l)$, and since $\left[\phi(x), \phi\left(x^{\prime}\right)\right]$ $=0$ if $x$ and $x^{\prime}$ are mutually spacelike, (4.3.37) is a direct corollary of (4. 3. 28) and (4.3. 29). See also pp. $942 \sim 943$.

Proposition 4. 3. 4. Let $d$ denote the exterior differentiation with respect to a. Then we have

$$
\begin{array}{ll}
\text { (4. 3. 39) } & d \phi_{l}(a)=\phi_{l+1}(a) d\left(-m a^{-}\right)+\phi_{l-1}(a) d\left(m a^{\top}\right), \\
(4.3 .40) & d \phi_{l}^{*}(a)=\phi_{l+1}^{*}(a) d\left(-m a^{-}\right)+\phi_{l-1}^{*}(a) d\left(m a^{\top}\right), \\
\text { (4. 3. 41) } & d \varphi_{B}(a ; l) \\
& =-2 \sin \pi l\left(\varphi_{B-l+1, l}^{*}(a ; l) d\left(-m a^{-}\right)+\varphi_{B-l, l-1}^{*}(a ; l) d\left(m a^{+}\right)\right) .
\end{array}
$$

Proof. (4.3.39) and (4.3.40) are easy. Since $\mathrm{Nr}$ is a linear map, it commutes with $d$. Namely, $\operatorname{Nr}\left(d \varphi_{B}(a ; l)\right)=d \operatorname{Nr}\left(\varphi_{B}(a ; l)\right)$. Then (4. 3. 41) follows directly from the definition of $\operatorname{Nr}\left(\varphi_{B}(a ; l)\right)$, (4.3.11).

In the following corollary, we shall abbreviate $v_{-l}\left(-(x-a)^{-}+i 0\right.$, $\left.(x-a)^{+}-i 0\right)$ to $v_{-i}[a]$, and so on.

Corollary 4. 3. 5. Assuming that $l \notin \mathbb{Z}$, we have

(4. 3. 42) $2 \sin \pi l \cdot \phi(x) \varphi_{l}^{B^{*}}(a ; l)$

$$
\begin{aligned}
= & \varphi_{B}(a ; l) v_{-l}[a]-\frac{\partial}{\partial\left(-m a^{-}\right)}-\varphi_{B}(a ; l) v_{-l+1}[a]+\cdots \\
& +\left\{\varphi_{B}(a ; l)+2 \sin \pi l \cdot \varphi_{B-l, l}(a ; l)\right\} v_{l}^{*}[a]+\cdots,
\end{aligned}
$$

(4. 3. 43) $2 \sin \pi l \cdot \phi^{*}(x) \varphi_{-l}^{B}(a ; l)$

$$
=\left\{\varphi_{B}(a ; l)+2 \sin \pi l \cdot \varphi_{B-l, l},(a ; l)\right\} v_{l}[a]+\cdots
$$




$$
+\varphi_{B}(a ; l) v_{-l}^{*}[a]-\frac{\partial}{\partial\left(m a^{+}\right)} \varphi_{B}(a ; l) v_{-l+1}^{*}[a]+\cdots
$$

When we consider the original operator $\varphi_{B}(a)$, we define $\varphi_{l}^{B}(a)$ and $\varphi_{B l_{1}, l_{2}}(a)$ by

$$
\begin{aligned}
& \operatorname{Nr}\left(\varphi_{l}^{B}(a)\right)=\phi_{l}(a) e^{\rho_{B}(a) / 2}, \\
& \operatorname{Nr}\left(\varphi_{B l_{1}, l_{2}}(a)\right)=\phi_{l_{1}}(a) \phi_{l_{2}}(a) e^{\rho_{B}(a) / 2},
\end{aligned}
$$

where $\phi_{l}(a)$ is given by (4.3.5) with the original $\phi(u)$ satisfying (4. 1.63). Then we have

$(4.3 .26)^{\prime} \phi(x) \varphi_{B}(a)$

$$
\begin{aligned}
& =\sum_{j=0}^{\infty} \varphi_{\frac{1}{2}+j}^{B}(a) v_{\frac{1}{2}+j}\left(-(x-a)^{-}+i 0,(x-a)^{+}-i 0\right) \\
& +\sum_{j=0}^{\infty} \varphi_{-\frac{1}{2}-j}^{B}(a) v_{\frac{1}{2}+j}^{*}\left(-(x-a)^{-}+i 0,(x-a)^{+}-i 0\right),
\end{aligned}
$$

$(4.3 .34)^{\prime} \phi(x) \varphi_{l^{\prime}}^{B}(a)$

$$
\begin{aligned}
& =\frac{1}{2 \sin \pi l^{\prime}} \varphi_{B}(a) v_{-l^{\prime}}\left(-(x-a)^{-}+i 0,(x-a)^{+}-i 0\right)
\end{aligned}
$$

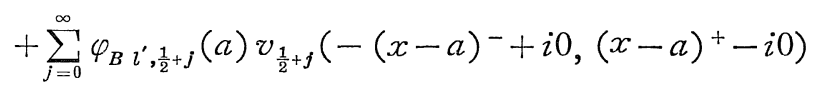

$$
\begin{aligned}
& -\frac{1}{2 \sin } \pi l^{\varphi^{\prime}} \varphi_{B}(a) v_{l^{\prime}}^{*}\left(-(x-a)^{-}+i 0,(x-a)^{+}-i 0\right) \\
& +\sum_{j=0}^{\infty} \varphi_{B} i^{\prime},-\frac{1}{2}-j(a) v_{\frac{1}{2}+j}^{*}\left(-(x-a)^{-}+i 0,(x-a)^{+}-i 0\right),
\end{aligned}
$$

for $l^{\prime} \equiv \frac{1}{2}(\bmod \mathscr{Z})$. (4.3.41), (4.3.42) and (4. 3. 43) must be slightly changed. Denoting $\varphi_{ \pm \frac{1}{2}}^{B}(a)$ by $\varphi_{ \pm}^{B}(a)$, we have

(4.3.45) $d \varphi_{B}(a)=-\varphi_{B \frac{1}{2}, \frac{1}{2}} d\left(-m a^{-}\right)-\varphi_{B-\frac{1}{2},-\frac{1}{2}} d\left(m a^{+}\right)$,

(4. 3. 46) $\phi(x) \varphi_{+}^{B}(a)$

$$
\begin{aligned}
= & \frac{1}{2} \varphi_{B}(a) v_{-\frac{1}{2}}[a]-\frac{\partial}{\partial\left(-m a^{-}\right)} \varphi_{B}(a) v_{\frac{1}{2}}[a]+\cdots \\
& +\left(-\frac{1}{2} \varphi_{B}(a)+\varphi_{B-\frac{1}{2}, \frac{1}{2}}(a)\right) v_{\frac{1}{2}}^{*}[a]+\cdots
\end{aligned}
$$


(4. 3. 47) $\phi(x) \varphi_{-}^{B}(a)$

$$
\begin{aligned}
= & \left(-\frac{1}{2} \varphi_{B}(a)+\varphi_{B-\frac{1}{2}, \frac{1}{2}}(a)\right) v_{\frac{1}{2}}[a]+\cdots \\
& +\frac{1}{2} \varphi_{B}(a) v_{-\frac{1}{2}}^{*}[a]-\frac{\partial}{\partial\left(m a^{+}\right)} \varphi_{B}(a) v_{\frac{1}{2}}^{*}[a]+\cdots .
\end{aligned}
$$

Now we state analogous results in the orthogonal case. We omit proofs, which are only refrains.

Remark. We have changed the definitions of $\varphi_{F}(a ; l), \varphi_{l^{\prime}}^{F}(a ; l)$ and $\varphi_{l^{\prime}}^{F^{*}}(a ; l)$ below from those in [7].

We start with $\psi(u)$ and $\psi^{*}(u)$ satisfying

$$
\begin{aligned}
& \left(\begin{array}{cc}
\left\langle\psi(u), \phi\left(u u^{\prime}\right)\right\rangle & \left\langle\psi(u), \phi^{*}\left(u^{\prime}\right)\right\rangle \\
\left\langle\psi^{*}(u), \phi\left(u^{\prime}\right)\right\rangle & \left\langle\psi^{*}(u), \phi^{*}\left(u^{\prime}\right)\right\rangle
\end{array}\right) \\
& =\left(\begin{array}{cc}
{\left[\psi(u), \phi\left(u^{\prime}\right)\right]_{+}} & {\left[\psi(u), \phi^{*}\left(u^{\prime}\right)\right]_{+}} \\
{\left[\psi^{*}(u), \phi\left(u^{\prime}\right)\right]_{+}} & {\left[\psi^{*}(u), \phi^{*}\left(u^{\prime}\right)\right]_{+}}
\end{array}\right) \\
& =\left(\begin{array}{cc}
0 & 2 \pi|u| \delta\left(u+u^{\prime}\right) \\
2 \pi|u| \delta\left(u+u^{\prime}\right) & 0
\end{array}\right),
\end{aligned}
$$

$$
\begin{aligned}
& \left(\begin{array}{cc}
\left\langle\psi(u) \phi\left(u^{\prime}\right)\right\rangle & \left\langle\psi(u) \psi^{*}\left(u^{\prime}\right)\right\rangle \\
\left\langle\psi^{*}(u) \psi\left(u^{\prime}\right)\right\rangle & \left\langle\psi^{*}(u) \psi^{*}\left(u^{\prime}\right)\right\rangle
\end{array}\right) \\
& \quad=\left(\begin{array}{cc}
0 & 2 \pi u_{+} \delta\left(u+u^{\prime}\right) \\
2 \pi u u_{+} \delta\left(u+u^{\prime}\right) & 0
\end{array}\right) .
\end{aligned}
$$

We set for $l \in \boldsymbol{C}$

$$
\begin{aligned}
& \phi_{l}(x)=\int d u(0+i u)^{l} e^{-i m\left(x^{-u+x^{+} u-1}\right)} \psi(u), \\
& \psi_{l}^{*}(x)=\int d u(0+i u)^{l} e^{-i m\left(x^{-} u+x^{+} u-1\right)} \phi^{*}(u) .
\end{aligned}
$$

$\left\langle\psi_{l}(x) \psi_{l^{\prime}}^{*}\left(x^{\prime}\right)\right\rangle=\left\langle\psi_{l}^{*}(x) \psi_{l^{\prime}}\left(x^{\prime}\right)\right\rangle$ is equal to $\left\langle\phi_{l}(x) \phi_{l^{\prime}}^{*}\left(x^{\prime}\right)\right\rangle$ given by (4. 3. 8).

We define $\rho_{F}(a ; l) \in \Lambda^{2}\left(W_{F}\right)$ by 
(4.3.52) $\rho_{F}(a ; l)=2 \iint \underline{d u} d u^{\prime} R_{F}\left(u, u^{\prime} ; l\right) \psi(u) \psi^{*}\left(u^{\prime}\right)$

$$
\times e^{-i m\left(a^{-}\left(u+u^{\prime}\right)+a^{+}\left(u^{-1}+u^{\prime-1}\right)\right)},
$$

where

(4. 3. 53) $\quad R_{F}\left(u, u^{\prime} ; l\right)=-2 i \cos \pi l\left(\frac{u-i 0}{u^{\prime}-i 0}\right)^{-l+\frac{1}{2}} \frac{\sqrt{u-i 0} \sqrt{u^{\prime}-i 0}}{u+u^{\prime}-i 0}$,

and define operators $\varphi_{F}(a ; l), \varphi_{l^{\prime}}^{F}(a ; l)$ and $\varphi_{l^{\prime}}^{F^{r}}(a ; l)$ by

$\operatorname{Nr}\left(\varphi_{F}(a ; l)\right)=\exp \left(\rho_{F}(a ; l) / 2\right)$,

$$
\operatorname{Nr}\left(\varphi_{l^{\prime}}^{F}(a ; l)\right)=\psi_{l^{\prime}}(a) \exp \left(\rho_{F}(a ; l) / 2\right),
$$

$$
\operatorname{Nr}\left(\varphi_{l^{\prime}}^{F^{+}}(a ; l)\right)=\psi_{l^{\prime}}^{*}(a) \exp \left(\rho_{F}(a ; l) / 2\right) .
$$

Note that

$$
R_{F}\left(u, u^{\prime} ; l\right)=-R_{F}\left(u^{\prime}, u ; 1-l\right)
$$

and that the anti-symmetric part of $R_{F}\left(u, u^{\prime} ; 0\right)$ coincides with the kernel in (4. 2.44).

In what follows we assume that $l \notin \frac{1}{2}+\mathbb{Z}$.

Theorem 4. 3. 6. The local operator expansion formulas read as follows.

$$
\begin{aligned}
& \left(\begin{array}{l}
\psi_{+}(x) \varphi_{F}(a ; l) \\
\psi_{-}(x) \varphi_{F}(a ; l)
\end{array}\right) \\
& \quad=\sum_{j=0}^{\infty} \varphi_{-l+1+j}^{F}(a ; l) \cdot w_{-l+1+j}\left(-(x-a)^{-}+i 0,(x-a)^{+}-i 0\right) \\
& \quad+\sum_{j=0}^{\infty} \varphi_{-l-j}^{F}(a ; l) \cdot w_{l+j}^{*}\left(-(x-a)^{-}+i 0,(x-a)^{+}-i 0\right)
\end{aligned}
$$

$(4.3 .59) \quad\left(\begin{array}{l}\psi_{+}^{*}(x) \varphi_{F}(a ; l) \\ \psi_{-}^{*}(x) \varphi_{F}(a ; l)\end{array}\right)$

$$
\begin{aligned}
= & \sum_{j=0}^{\infty} \varphi_{l+j}^{F *}(a ; l) \cdot w_{l+j}\left(-(x-a)^{-}+i 0,(x-a)^{+}-i 0\right) \\
& +\sum_{j=0}^{\infty} \varphi_{l-1-j}^{F *}(a ; l) \cdot w_{-l+1+j}^{*}\left(-(x-a)^{-}+i 0,(x-a)^{+}-i 0\right) .
\end{aligned}
$$


$\left(\begin{array}{l}\varphi_{F}(a ; l) \psi_{+}(x) \\ \varphi_{F}(a ; l) \psi_{-}(x)\end{array}\right)$ and $\left(\begin{array}{l}\varphi_{F}(a ; l) \psi_{+}^{*}(x) \\ \varphi_{F}(a ; l) \psi_{-}^{*}(x)\end{array}\right)$ are given by the right hand sides of (4.3.58) and (4.3.59) respectively, with $\mp(x-a)^{\mp} \pm i 0$ replaced by $\mp(x-a)^{\mp} \mp i 0$.

We define the following operators.

(4. 3. 60) $\quad \operatorname{Nr}\left(\varphi_{F} l_{1}, l_{2}(a ; l)\right)=\psi_{l_{1}}(a) \phi_{l_{2}}(a) e^{\rho_{F}(a ; l) / 2}$.

(4.3.61) $\operatorname{Nr}\left(\varphi_{F^{\prime}}{ }_{l_{1}, l_{2}}^{*}(a ; l)\right)=\phi_{l_{1}}^{*}(a) \phi_{l_{2}}^{*}(a) e^{\rho_{F}(a ; l) / 2}$.

(4.3.62) $\operatorname{Nr}\left(\varphi_{F} l_{1}, l_{2}(a ; l)\right)=-\operatorname{Nr}\left(\varphi_{F}^{*} i_{2}, l_{1}(a ; l)\right)$

$$
==\psi_{l_{1}}(a) \psi_{l_{2}}^{*}(a) e^{\rho_{F}(a ; l) / 2}=-\psi_{l_{2}}^{*}(a) \psi_{l_{1}}(a) e^{\rho_{F}(a ; l) / 2} .
$$

Theorem 4. 3. 7. Assuming $l, l^{\prime} \notin \frac{1}{2}+\mathbb{Z}$, we have

(4. 3. 63) $\left(\begin{array}{l}\psi_{+}(x) \varphi_{l^{\prime}}^{F^{\prime}}(a ; l) \\ \psi_{-}(x) \varphi_{l^{\prime}}^{F}(a ; l)\end{array}\right)$

$$
\begin{aligned}
= & \sum_{j=0}^{\infty} \varphi_{F-l+1+j, l^{\prime}}(a ; l) w_{-l+1+j}\left(-(x-a)^{-}+i 0,(x-a)^{+}-i 0\right) \\
& +\sum_{j=0}^{\infty} \varphi_{F-l-j, l^{\prime}}(a ; l) w_{l+j}^{*}\left(-(x-a)^{-}+i 0,(x-a)^{+}-i 0\right),
\end{aligned}
$$

$(4.3 .64)\left(\begin{array}{l}\psi_{+}(x) \varphi_{l^{\prime}}^{F^{+}}(a ; l) \\ \psi_{-}(x) \varphi_{l^{\prime}}^{F^{*}}(a ; l)\end{array}\right)$

$$
\begin{aligned}
= & \frac{i}{2 \cos \pi l^{\prime}} \varphi_{F}(a ; l) w_{-l^{\prime}}\left(-(x-a)^{-}+i 0,(x-a)^{+}-i 0\right) \\
& +\sum_{j=0}^{\infty} \varphi_{F-l+1+j, l^{\prime}}^{*}(a ; l) w_{-l+1+j}\left(-(x-a)^{-}+i 0,(x-a)^{+}-i 0\right) \\
& -\frac{i}{2 \cos \pi l^{\prime}} \varphi_{F}(a ; l) w_{l^{\prime}}^{*}\left(-(x-a)^{-}+i 0,(x-a)^{+}-i 0\right) \\
& +\sum_{j=0}^{\infty} \varphi_{F-l-j, l^{\prime}}(a ; l) w_{l+j}^{*}\left(-(x-a)^{-}+i 0,(x-a)^{+}-i 0\right),
\end{aligned}
$$

(4. 3. 65) $\quad\left(\begin{array}{l}\psi_{+}^{*}(x) \varphi_{l^{\prime}}^{F^{*}}(a ; l) \\ \psi_{-}^{*}(x) \varphi_{l^{\prime}}^{F^{+}}(a ; l)\end{array}\right)$

$$
=\sum_{j=0}^{\infty} \varphi_{F} l_{+j}^{*} l^{\prime}(a ; l) w_{l+j}\left(-(x-a)^{-}+i 0,(x-a)^{+}-i 0\right)
$$




$$
+\sum_{j=0}^{\infty} \varphi_{F} \underset{l-1-j}{*} l^{\prime}(a ; l) w_{-l+1+j}^{*}\left(-(x-a)^{-}+i 0,(x-a)^{+}-i 0\right),
$$

$(4.3 .66) \quad\left(\begin{array}{l}\psi_{+}^{*}(x) \varphi_{l^{\prime}}^{F}(a ; l) \\ \psi_{-}^{*}(x) \varphi_{l^{\prime}}^{F}(a ; l)\end{array}\right)$

$$
\begin{aligned}
& =\frac{i}{2 \cos \pi l^{\prime}} \varphi_{F}(a ; l) w_{-l^{\prime}}\left(-(x-a)^{-}+i 0,(x-a)^{+}-i 0\right) \\
& +\sum_{j=0}^{\infty} \varphi_{F} \underset{i+j}{*} l^{\prime}(a ; l) w_{l+j}\left(-(x-a)^{-}+i 0,(x-a)^{+}-i 0\right) \\
& -\frac{i}{2 \cos \pi l^{\prime}} \varphi_{F}(a ; l) w_{l^{\prime}}^{*}\left(-(x-a)^{-}+i 0,(x-a)^{+}-i 0\right) \\
& +\sum_{j=0}^{\infty} \varphi_{F} l_{l-1-j}^{*} l^{\prime}(a ; l) w_{-l+1+j}^{*}\left(-(x-a)^{-}+i 0,(x-a)^{+}-i 0\right) . \\
& -\left(\begin{array}{l}
\varphi_{l^{\prime}}^{F}(a ; l) \psi_{+}(x) \\
\varphi_{l^{\prime}}^{F}(a ; l) \psi_{-}(x)
\end{array}\right),-\left(\begin{array}{l}
\varphi_{l^{\prime}}^{F^{\prime}}(a ; l) \psi_{+}(x) \\
\varphi_{l^{\prime}}^{F^{*}}(a ; l) \psi_{-}(x)
\end{array}\right) \text {, etc. are given by the right } \\
& \text { hand side of (4.3.63) and (4.3.64), etc., with } \mp(x-a)^{\mp} \pm i 0 \text { re- } \\
& \text { placed by } \mp(x-a)^{\mp} \mp i 0 \text {. }
\end{aligned}
$$

Corollary 4. 3. 8. We have the following commutation relations in the region where $x$ and a are mutually spacelike.

$(4.3 .67) \quad \varphi_{F}(a ; l) \psi_{ \pm}(x)$

$$
=\left\{\begin{array}{lll}
\psi_{ \pm}(x) \varphi_{F}(a ; l) & \text { if } & x^{+}>a^{+} \text {and } x^{-}<a^{-} \\
-e^{2 \pi i l} \psi_{ \pm}(x) \varphi_{F}(a ; l) & \text { if } & x^{+}<a^{+} \text {and } x^{-}>a^{-}
\end{array}\right.
$$

(4.3.68) $\varphi_{F}(a ; l) \psi_{ \pm}^{*}(x)$

$$
=\left\{\begin{array}{lll}
\psi_{ \pm}^{*}(x) \varphi_{F}(a ; l) & \text { if } & x^{+}>a^{+} \text {and } x^{-}<a^{-} \\
-e^{-2 x i l} \psi_{ \pm}^{*}(x) \varphi_{F}(a ; l) & \text { if } & x^{+}<a^{+} \text {and } x^{-}>a^{-}
\end{array}\right.
$$

If $l^{\prime} \equiv-l(\bmod \mathbb{Z})$, we have

(4.3.69) $\varphi_{l^{\prime}}^{F}(a ; l) \psi_{ \pm}(x)$

$$
\begin{gathered}
=\left\{\begin{array}{lll}
-\psi_{ \pm}(x) \varphi_{l^{\prime}}^{F}(a ; l) & \text { if } & x^{+}>a^{+} \text {and } x^{-}<a^{-} \\
e^{2 \pi i l} \psi_{ \pm}(x) \varphi_{l^{\prime}}^{F}(a ; l) & \text { if } & x^{+}<a^{+} \text {and } x^{-}>a^{-},
\end{array}\right. \\
\varphi_{l^{\prime}}^{F}(a ; l) \psi_{ \pm}^{*}(x) \\
=\left\{\begin{array}{lll}
-\psi_{ \pm}^{*}(x) \varphi_{l^{\prime}}^{F}(a ; l) & \text { if } & x^{+}>a^{+} \text {and } x^{-}<a^{-} \\
e^{-2 \pi i l} \psi_{ \pm}^{*}(x) \varphi_{l^{\prime}}^{F}(a ; l) & \text { if } & x^{+}<a^{+} \text {and } x^{-}>a^{-}
\end{array}\right.
\end{gathered}
$$


If $l^{\prime} \equiv l(\bmod Z)$, we have

(4. 3. 70) $\varphi_{l}^{F^{*}}(a ; l) \psi_{ \pm}(x)$

$$
\begin{gathered}
\left\{\begin{array}{lll}
-\psi_{ \pm}(x) \varphi_{l^{\prime}}^{F^{*}}(a ; l) & \text { if } & x^{+}>a^{+} \text {and } x^{-}<a^{-} \\
e^{2 \pi i l} \psi_{ \pm}(x) \varphi_{l^{\prime}}^{F^{*}}(a ; l) & \text { if } & x^{+}<a^{+} \text {and } x^{-}>a^{-},
\end{array}\right. \\
\varphi_{l^{\prime}}^{F^{\prime}}(a ; l) \psi_{ \pm}^{*}(x) \\
=\left\{\begin{array}{lll}
-\psi_{ \pm}^{*}(x) \varphi_{l^{\prime}}^{F^{\prime}}(a ; l) & \text { if } & x^{+}>a^{+} \text {and } x^{-}<a^{-} \\
e^{-2 \pi i l} \psi_{ \pm}^{*}(x) \varphi_{l^{\prime}}^{F^{\prime}}(a ; l) & \text { if } & x^{+}<a^{+} \text {and } x^{-}>a^{-} .
\end{array}\right.
\end{gathered}
$$

\section{Proposition 4. 3.9.}

$$
d \psi_{l}(a)=\psi_{l+1}(a) d\left(-m a^{-}\right)+\psi_{l-1}(a) d\left(m a^{+}\right),
$$

$$
d \psi_{l}^{*}(a)=\psi_{l_{+1}}^{*}(a) d\left(-m a^{-}\right)+\psi_{l-1}^{*}(a) d\left(m a^{+}\right),
$$

$$
\begin{aligned}
d \varphi_{F}(a ; l)= & -2 i \cos \pi l\left\{\varphi_{F-l+1, l}(a ; l) d\left(-m a^{-}\right)\right. \\
& \left.+\varphi_{F-l, l-1}^{*}(a ; l) d\left(m a^{+}\right)\right\} .
\end{aligned}
$$

Theorem 4. 3. 10. Denoting $w_{-l}\left(-(x-a)^{-}+i 0,(x-a)^{+}-i 0\right)$ by $w_{-l}[a]$ and so on, we have

$$
\begin{aligned}
- & 2 i \cos \pi l\left(\begin{array}{l}
\psi_{+}(x) \varphi_{l}^{F^{*}}(a ; l) \\
\psi_{-}(x) \varphi_{l}^{F^{*}}(a ; l)
\end{array}\right) \\
= & \varphi_{F}(a ; l) w_{-l}[a]+\frac{\partial}{\partial\left(-m a^{-}\right)} \varphi_{F}(a ; l) w_{-l+1}[a]+\cdots \\
& -\left\{\varphi_{F}(a ; l)+2 i \cos \pi l \cdot \varphi_{F-l, l}^{*}(a ; l)\right\} w_{l}^{*}[a]+\cdots,
\end{aligned}
$$

(4. 3. 75) $\quad-2 i \cos \pi l\left(\begin{array}{l}\psi_{+}^{*}(x) \varphi_{-l}^{F}(a ; l) \\ \psi_{-}^{*}(x) \varphi_{-l}^{F}(a ; l)\end{array}\right)$

$$
\begin{aligned}
= & \left\{\varphi_{F}(a ; l)+2 i \cos \pi l \cdot \varphi_{F-l, l}^{*}(a ; l)\right\} w_{l}[a]+\cdots \\
& -\varphi_{F}(a ; l) w_{-l}^{*}[a]-\frac{\partial}{\partial\left(m a^{+}\right)} \varphi_{F}(a ; l) w_{-l+1}^{*}[a]+\cdots .
\end{aligned}
$$

When we consider the original operator $\varphi_{F}(a)$, we define $\varphi_{l}^{F}(a)$, $\varphi_{F l_{1}, l_{2}}(a)$ by

$$
\text { (4. 3. 76) } \quad \begin{array}{ll}
\operatorname{Nr}\left(\varphi_{l}^{F}(a)\right)=\phi_{l}(a) e^{\rho_{F}(a) / 2}, \\
& \operatorname{Nr}\left(\varphi_{l_{1}, l_{2}}^{F}(a)\right)=\phi_{l_{1}}(a) \phi_{l_{2}}(a) e^{\rho_{F}(a) / 2},
\end{array}
$$


where $\psi_{l}(x)$ is given by (4.3.50) with the original $\psi(u)$ satisfying (4. 2. 42). We abbreviate $\varphi_{0}^{F}(a)$ to $\varphi^{F}(a)$. Since $R_{F}\left(u, u^{\prime} ; 0\right)$ is not antisymmetric, the local operator expansion formula for $\varphi_{F}(a)$ is slightly different from that of $\varphi_{F}(a ; 0)$. The results are as follows. See also $[5]$.

$$
\begin{gathered}
\left(\begin{array}{l}
\psi_{+}(x) \varphi_{F}(a) \\
\psi_{-}(x) \varphi_{F}(a)
\end{array}\right)=\frac{1}{2} \varphi^{F}(a)\left(w_{0}[a]+w_{0}^{*}[a]\right) \\
+\sum_{j=1}^{\infty}\left(\varphi_{j}^{F}(a) w_{j}[a]+\varphi_{-j}^{F}(a) w_{j}^{*}[a]\right) .
\end{gathered}
$$

$$
\begin{aligned}
& \left(\begin{array}{l}
\psi_{+}(x) \varphi^{F}(a) \\
\psi_{-}(x) \varphi^{F}(a)
\end{array}\right)=\frac{i}{2} \varphi_{F}(a)\left(w_{0}[a]-w_{0}^{*}[a]\right) \\
& \left.\quad+\sum_{j=1}^{\infty} \varphi_{F j, 0}(a) w_{j}[a]+\varphi_{F-j, 0}(a) w_{j}^{*}[a]\right) .
\end{aligned}
$$

$$
\begin{aligned}
& d \varphi_{F}(a)=-i \varphi_{F^{1}, 0}(a) d\left(-m a^{-}\right)+i \varphi_{F-1,0}(a) d\left(m a^{+}\right) \\
& d \varphi^{F}(a)=\varphi_{1}^{F}(a) d\left(-m a^{-}\right)+\varphi_{-1}^{F}(a) d\left(m a^{+}\right) .
\end{aligned}
$$

\section{§4. 4. Wave Functions and $\tau$ Functions}

We shall now investigate the properties of various vacuum expectation values, involving operators $\varphi_{B}(a ; l), \varphi_{l^{\prime}}^{B}(a ; l)$ and $\varphi_{l^{\prime}}^{B r}(a ; l)$ (or their orthogonal versions) and the free fields $\phi(x), \phi^{*}(x)$ (or $\psi(x), \psi^{*}(x)$ ). Our goal is to relate their Euclidean continuations to the wave functions having specific monodromy properties, and thereby establish the connection with the deformation theory expounded in the previous Chapter III.

Our argument is twofold. The present Section 4.4 is the formal part, in which we shall carry out the above program by making use of the properties of field operators in class $G$. The parameters $\Lambda=\left(\lambda_{\mu \nu}\right)$ introduced in Section 3.2 naturally arise in this context. However neither the positive definiteness of $A$ nor the reality of the exponents $l_{\nu}$ of local monodromy are necessary here, at least formally. The next Section 4.5 is devoted to the analytical part. Using the product formula we shall derive infinite series expansions for the wave- and $\tau$-functions, and show that they are convergent in the complex domain (Propositions 4.5.1 4. 5. 3, Theorem 4. 5. 4). In this way the formulas derived in this Section 4.4 are recovered rigorously. 
Let $x, x^{*}, a_{1}, \cdots, a_{n}$ denote mutually spacelike points of $X^{\mathrm{Min}}$. Consider the following vacuum expectation values, to which we shall hereafter refer as (Minkowski) Bose wave functions:

$$
\begin{aligned}
& w_{B}\left(x^{*}, x ; L\right)=\left\langle\phi^{*}\left(x^{*}\right) \varphi_{B}\left(a_{1} ; l_{1}\right) \cdots \varphi_{B}\left(a_{n} ; l_{n}\right) \phi(x)\right\rangle \\
& w_{B \nu}(x ; L)=\left\langle\varphi_{B}\left(a_{1} ; l_{1}\right) \cdots \varphi_{l_{\nu}}^{B^{*}}\left(a_{\nu} ; l_{\nu}\right) \cdots \varphi_{B}\left(a_{n} ; l_{n}\right) \phi(x)\right\rangle \\
& w_{B \nu}^{*}\left(x^{*} ; L\right)=\left\langle\phi^{*}\left(x^{*}\right) \varphi_{B}\left(a_{1} ; l_{1}\right) \cdots \varphi_{-l_{\nu}}^{B}\left(a_{\nu} ; l_{\nu}\right) \cdots \varphi_{B}\left(a_{n} ; l_{n}\right)\right\rangle .
\end{aligned}
$$

Likewise we define the (Minkowski) Fermi wave functions $w_{F}\left(x^{*}, x ; L\right)$ $=\left(w_{F \varepsilon \varepsilon \varepsilon}\left(x^{*}, x ; L\right)\right)_{\varepsilon, \varepsilon^{*}= \pm}, w_{F_{\nu}}(x ; L)={ }^{t}\left(w_{F \nu+}(x ; L), w_{F \nu}(x ; L)\right) \quad$ and $w_{F_{\nu}}^{*}\left(x^{*} ; L\right)={ }^{t}\left(w_{F^{\nu+}}^{*}\left(x^{*} ; L\right), w_{F^{\nu}-}^{*}\left(x^{*} ; L\right)\right)$ by

$$
\begin{aligned}
& w_{F^{*} \varepsilon}\left(x^{*}, x ; L\right)=\left\langle\psi_{\varepsilon^{*}}^{F^{*}}\left(x^{*}\right) \varphi_{F}\left(a_{1} ; l_{1}\right) \cdots \varphi_{F}\left(a_{n} ; l_{n}\right) \psi_{\varepsilon}(x)\right\rangle \\
& { }_{F_{\nu \pm}}(x ; L)=\left\langle\varphi_{F}\left(a_{1} ; l_{1}\right) \cdots \varphi_{l_{\nu}}^{F^{*}}\left(a_{\nu} ; l_{\nu}\right) \cdots \varphi_{F}\left(a_{n} ; l_{n}\right) \psi_{ \pm}(x)\right\rangle \\
& w_{F^{\prime} \pm}^{*}\left(x^{*} ; L\right)=\left\langle\psi_{ \pm}^{*}\left(x^{*}\right) \varphi_{F}\left(a_{1} ; l_{1}\right) \cdots \varphi_{-l_{\nu}}^{F}\left(a_{\nu} ; l_{\nu}\right) \cdots \varphi_{F}\left(a_{n} ; l_{n}\right)\right\rangle .
\end{aligned}
$$

We also deal with the Bose and Fermi $\tau$ functions $^{(*)}$ defined by

$$
\begin{aligned}
& \tau_{B}(L)=\tau_{B}\left(a_{1}, \cdots, a_{n} ; L\right)=\left\langle\varphi_{B}\left(a_{1} ; l_{1}\right) \cdots \varphi_{B}\left(a_{n} ; l_{n}\right)\right\rangle \\
& \tau_{F}(L)=\tau_{F}\left(a_{1}, \cdots, a_{n} ; L\right)=\left\langle\varphi_{F}\left(a_{1} ; l_{1}\right) \cdots \varphi_{F}\left(a_{n} ; l_{n}\right)\right\rangle .
\end{aligned}
$$

Obviously (4.4.1) or (4.4.2), regarded as functions of $x$ and $x^{*}$, are solutions of the 2-dimensional Klein-Gordon equation (4.4.1) (with $n=2$ ) or the Dirac equation (4.2.42) respectively. (*) Their local properties are known immediately by applying Theorems 4.3.2, 4.3.3, 4.3.6 and 4. 3. 7. For instance assume $x^{+}>a_{\nu+1}^{+}>a_{\nu+2}^{+}>\cdots>a_{n}^{+}$. From (4.3.28), $\phi(x)$ commutes with $\varphi_{B}\left(a_{\mu} ; l_{\mu}\right) \quad(\nu+1 \leqq \mu \leqq n)$ in this region. Therefore we have $w_{B}\left(x^{*}, x ; L\right)=\left\langle\phi^{*}\left(x^{*}\right) \varphi_{B}\left(a_{1} ; l_{1}\right) \cdots \varphi_{B}\left(a_{\nu} ; l_{\nu}\right) \phi(x) \varphi_{B}\left(a_{\nu+1} ; l_{\nu+1}\right) \cdots\right.$ $\left.\varphi_{B}\left(a_{n} ; l_{n}\right)\right\rangle$ there. Applying (4.3.26) for $\varphi_{B}\left(a_{\nu} ; l_{\nu}\right) \phi(x)$ we then obtain the local expansion for $w_{B}\left(x^{*}, x ; L\right)$ at $a_{\nu}$. Hereafter, in writing down the local expansions, we assume $x^{*+}>a_{1}^{+}>\cdots>a_{n}^{+}$for $w_{B}, w_{B \nu}, w_{F}, w_{F_{\nu}}$ and $x^{*+}<a_{1}^{+}<\cdots<a_{n}^{+}$for $w_{B \nu}^{*}, w_{F_{\nu}}^{*}$. Otherwise the coefficients of the expansions should be modified according to the commutation relations

(*) For the lack of the time-ordering sign, the expectation values (4.4.3) may more properly be called the Wightmann functions. Regarding the microcausality condition for the $\varphi$-fields (see $\$ 4.5$ ) we may confuse these two notions, since we are working in the spacelike-separated region of arguments.

(**) In the case of $w_{F}\left(x^{*}, x ; L\right)$, its transpose satisfies (4.2.42) with respect to $x^{*}$. 
(4. 3. 28), (4.3.29), (4.3.37), (4.3.38) or their Fermi versions.

$(4.4 .4)_{0} \quad w_{B}\left(x^{*}, x ; L\right)=\frac{1}{\pi} \tau_{B}(L) \cdot \widetilde{v}_{0}\left[x^{*}\right]$

$$
\begin{gathered}
+\sum_{j=1}^{c} c_{j}^{(0)}\left(w_{B}\right) \cdot v_{j}\left[x^{*}\right]+\sum_{j=0}^{c} c_{j}^{*(0)}\left(w_{B}\right) \cdot v_{j}^{*}\left[x^{*}\right], \\
\left\{\begin{array}{l}
c_{j}^{(0)}\left(w_{B}\right)=\left\langle: \phi^{*}\left(x^{*}\right) \phi_{j}\left(x^{*}\right): \varphi_{B}\left(a_{1} ; l_{1}\right) \cdots \varphi_{B}\left(a_{n} ; l_{n}\right)\right\rangle(j \in \mathbb{Z}) \\
c_{j}^{*(0)}\left(w_{B}\right)=c_{-j}^{(0)}\left(w_{B}\right) \quad(j \geqq 0) .
\end{array}\right.
\end{gathered}
$$

(4. 4. 4), $w_{B}\left(x^{*}, x ; L\right)$

$$
\begin{array}{r}
=\sum_{j=1}^{\infty} c_{-l_{\nu}+j}^{(\nu)}\left(w_{B}\right) \cdot v_{-l_{\nu}+j}\left[a_{\nu}\right]+\sum_{j=0}^{\infty} c_{l_{\nu}+j}^{*(\nu)}\left(w_{B}\right) \cdot v_{l_{\nu}+j}^{*}\left[a_{\nu}\right], \\
\left\{\begin{array}{cc}
c_{-l_{\nu}+j}^{(\nu)}\left(w_{B}\right)=\left\langle\phi^{*}\left(x^{*}\right) \varphi_{B}\left(a_{1} ; l_{1}\right) \cdots \varphi_{-l_{\nu}+j}^{B}\left(a_{\nu} ; l_{\nu}\right) \cdots \varphi_{B}\left(a_{n} ; l_{n}\right)\right\rangle \\
(j \in \mathbb{Z}) \\
c_{l_{\nu}+j}^{*(\nu)}\left(w_{B}\right)=c_{-l_{\nu}-j}^{(\nu)}\left(w_{B}\right) \quad(j \geqq 0) .
\end{array}\right.
\end{array}
$$

$(4.4 .5)_{\mu \nu} w_{B \mu}(x ; L)$

$$
\begin{gathered}
=-\frac{\tau_{B}(L)}{2 \sin \pi l_{\mu}} \hat{o}_{\mu \nu} v_{-l_{\nu}}\left[a_{\nu}\right]+\sum_{j=1}^{\infty} c_{-l_{\nu}+j}^{(\nu)}\left(w_{B \mu}\right) \cdot v_{-l_{\nu}+j}\left[a_{\nu}\right] \\
+\sum_{j=0}^{\infty} c_{l_{\nu}+j}^{*(\nu)}\left(w_{B \mu}\right) \cdot v_{l_{\nu}+j}^{*}\left[a_{\nu}\right], \\
\qquad \begin{aligned}
c_{-l_{\nu}+j}^{(\nu)}\left(w_{B \mu}\right)=\left\langle\varphi_{B}\left(a_{1} ; l_{1}\right) \cdots \varphi_{l_{\mu}}^{B *}\left(a_{\mu} ; l_{\mu}\right) \cdots \varphi_{-l_{\nu}+j}^{B}\right. & \left.\left(a_{\nu} ; l_{\nu}\right) \cdots \varphi_{B}\left(a_{n} ; l_{n}\right)\right\rangle \\
=\left\langle\varphi_{B}\left(a_{1} ; l_{1}\right) \cdots \varphi_{B} l_{l_{\mu},-l_{\mu}+j}^{*}\left(a_{\mu} ; l_{\mu}\right)\right. & \left.\cdots \varphi_{B}\left(a_{n} ; l_{n}\right)\right\rangle \\
& (\mu=\nu, j \in \mathbb{Z}) \\
c_{l_{\nu}+j}^{*(\nu)}\left(\tau_{B \mu}\right)=-\frac{\tau_{B}(L)}{2 \sin \pi l_{\mu}} \delta_{j 0} \delta_{\mu \nu}+c_{-l_{\nu}-j}^{(\nu)}\left(w_{B \mu}\right) & (j \geqq 0)
\end{aligned}
\end{gathered}
$$

where $v_{l}[a]=v_{l}\left(-(x-a)^{-}-i 0,(x-a)^{+}+i 0\right)$, etc.

$(4.4 .5)_{\mu \nu}^{*} \quad \tau_{B \mu}^{*}\left(x^{*} ; L\right)=\sum_{j=0}^{c_{\nu}} c_{l_{\nu}+j}^{(\nu)}\left(w_{B / l}^{*}\right) \cdot v_{l_{\nu}+j}^{\prime}\left[a_{\nu}\right]$

$$
\begin{gathered}
+\frac{\tau_{B}(L)}{2 \sin \pi l_{\mu}} \delta_{\mu \nu} v_{-l_{\nu}}^{* \prime}\left[a_{\nu}\right]+\sum_{j=1}^{\infty} c_{-l_{\nu}+j}^{*(\nu)}\left(w_{B \mu}^{*}\right) \cdot v_{-l_{\nu}+j}^{* \prime}\left[a_{\nu}\right] \\
\left\{\begin{array}{cc}
c_{l_{\nu}+j}^{(\nu)}\left(w_{B \mu}^{*}\right)=-\frac{\tau_{B}(L)}{2 \sin \pi l_{\mu}} \delta_{j 0} \delta_{\mu \nu}+c_{-l_{\nu}-j}^{*(\nu)}\left(w_{B \mu}\right) \quad(j \geqq 0) \\
c_{-l_{\nu}+j}^{*(\nu)}\left(w_{B, \mu}^{*}\right)=\left\langle\varphi_{B}\left(a_{1} ; l_{1}\right) \cdots \varphi_{-l_{\mu}}^{B}\left(a_{\mu} ; l_{\mu}\right) \cdots \varphi_{l_{\nu}-j}^{B *}\left(a_{\nu} ; l_{\nu}\right) \cdots \varphi_{B}\left(a_{n} ; l_{n}\right)\right\rangle \\
(\mu \neq \nu, j \in \mathbb{Z}) \\
=\left\langle\varphi_{B}\left(a_{1} ; l_{1}\right) \cdots \varphi_{B-l_{\mu}, l_{2}{ }^{*} j}\left(a_{\mu} ; l_{\mu}\right) \cdots \varphi_{B}\left(a_{n} ; l_{n}\right)\right\rangle \\
(\mu=\nu, j \in \mathbb{Z})
\end{array}\right.
\end{gathered}
$$


where $v_{l}^{\prime}[a]=v_{l}\left(-\left(x^{*}-a\right)^{-}+i 0,\left(x^{*}-a\right)^{+}-i 0\right)$, etc. In deriving (4. 4. 4) o we have used the formula

$$
\begin{aligned}
& e^{-i m\left(x^{-} v+x^{+} v^{-1}\right)}=\sum_{j=-\infty}^{+\infty}(0+i u)^{j} v_{j}\left(-x^{-}, x^{+}\right),(*) \\
& \phi(x)=\sum_{j=-\infty}^{+\infty} \phi_{j}\left(x^{*}\right) v_{j}\left[x^{*}\right] .
\end{aligned}
$$

Set

(4. 4.7) $\quad Y^{n, \boldsymbol{C}}=\left\{\left(x_{1}, \cdots, x_{n}\right) \in\left(X^{\boldsymbol{C}}\right)^{n} \mid \operatorname{Im}\left(x_{\mu}-x_{\nu}\right)^{ \pm}<0 \quad(1 \leqq \mu<\nu \leqq n)\right\}$,

(4. 4. 8) $\quad Y^{n \text { Euc }}=Y^{n, \boldsymbol{C}} \cap\left(X^{\mathrm{Euc}}\right)^{n}$.

We shall see in Section 4.5 that the wave- and $\tau$-functions are analytically prolongable to the domain of the form (4.4.7) (e.g. $\left(x^{*}, a_{1}, \cdots, a_{n}, x\right)$ $\in Y^{n+2, C}$ for $\left.w_{B}\left(x^{*}, x ; L\right)\right)$, in particular to the corresponding Euclidean region (4.4.8). Moreover they are bounded in the $\operatorname{limit} \operatorname{Im}\left(x^{*}-a_{\nu}\right)=$ $\rightarrow-\infty, \operatorname{Im}(x-a)^{ \pm} \rightarrow+\infty \quad(1 \leqq \nu \leqq n)$ and $\operatorname{Im}\left(a_{\mu}-a_{\nu}\right)^{ \pm} \rightarrow-\infty \quad(1 \leqq \mu<\nu$ $\leqq n)$. Hereafter in the Euclidean space $X^{\text {Euc }}$ we often write the variables $\left(-x^{-}, x^{+}\right),\left(-x^{*-}, x^{*+}\right)$ and $\left(-a_{\nu}^{-}, a_{\nu}^{-}\right)$as $(z, \bar{z}),\left(z^{*}, \bar{z}^{*}\right)$ and $\left(a_{\nu}, \bar{a}_{\nu}\right)$ respectively. The Euclidean continuation of $w_{B}\left(x^{*}, x ; L\right)$ is also denoted by the same letter $w_{B}\left(z^{*}, z ; L\right)$, and similarly for $w_{B \nu}, w_{B \nu}^{*}$ or $\tau_{B}$.

Set now

$$
\begin{aligned}
& \widehat{\omega}_{B}\left(x^{*}, x ; L\right)=\pi w_{B}\left(x^{*}, x ; L\right) / \tau_{B}(L), \\
& \widehat{w}_{B \mu}(x ; L)=2 \sin \pi l_{\mu} \cdot w_{B \mu}(x ; L) / \tau_{B}(L), \\
& \widehat{w}_{B \mu}^{*}\left(x^{*} ; L\right)=2 \sin \pi l_{\mu} \cdot w_{B \mu}^{*}\left(x^{*} ; L\right) / \tau_{B}(L) .
\end{aligned}
$$

The local expansions (4.4.4) $)_{0} \sim(4.4 .5)_{\mu \nu}^{*}$ then imply that the Euclidean continuations of (4.4.9) are multi-valued on $X^{\prime}=X^{\text {Euc }}-\left\{\left(a_{\nu}, \bar{a}_{\nu}\right)\right\}_{\nu}$ $=1, \ldots, n$, having the monodromic property (3.2.3). This is in fact an immediate consequence of the commutation relations (4.3.28), (4.3.29), (4. 3.37) and (4.3.38) among the $\varphi$ - and the free $\phi$-fields (cf. $\S 2.2$ ).

Summing up we have

Proposition 4. 4. 1. For $l_{1}, \cdots, l_{n} \in \mathbb{C}-\mathbb{Z}$, the Euclidean continua

(*) $v_{j}^{*}=v_{-j}$ for all $j \in Z$. 
tions of $\widehat{w}_{B}\left(x^{*}, x ; L\right), \widehat{w}_{B \mu}(x ; L) \quad(1 \leqq \mu \leqq n) \quad$ and $\quad \widehat{\boldsymbol{w}}_{B \mu}^{*}\left(x^{*} ; L\right) \quad(1 \leqq \mu$ $\leqq n)$ provide linearly independent elements of $W_{B, z^{2}, \cdots, a_{1}, \cdots, a_{n}}^{0, l_{n}, \text { strict }}$ and of $W_{B, a_{1}, \cdots, a_{n}}^{* l_{1}, \cdots, l_{n}, \text { strict }}$ respectively. They are canonical in the sense $\tilde{c}_{0}^{(0)}\left(\widehat{\mathscr{w}}_{B}\right)$ $=1, c_{-l_{\nu}}^{(\nu)}\left(\hat{\boldsymbol{w}}_{B}\right)=0, c_{-l_{\nu}}^{(\nu)}\left(\widehat{\boldsymbol{w}}_{B \mu}\right)=\delta_{\mu \nu}$ and $c_{-\nu_{\nu}}^{*(\nu)}\left(\widehat{\boldsymbol{w}}_{B \mu}^{*}\right)=\delta_{\mu \nu}$. In paricular if $k<l_{1}, \cdots, l_{n}<k+1$ for sone $k \in \mathbb{Z}$, they coincide with those $v_{0}\left(z^{*}, z ; L\right)$, $v_{\mu}(z ; L)$ and $\overline{v_{\mu}}\left(z^{*} ; L\right)$ constructed in Chapter III.

Remark. Strictly speaking we must impose a restriction on the region of $x^{*}, x, a_{\nu}$ and $l_{\nu}$. For the precise statement see Section 4.5.

In particular, from (4.4.4) $)_{0} \sim(4.4 .5)_{\mu \nu}$ we obtain the following expressions for $\alpha_{0 \nu}\left(z^{*} ; L\right)$, etc. in Section 3.2 in terms of field operators (cf. (3.2.12)):

$$
\begin{gathered}
\alpha_{0 \nu}\left(x^{*} ; L\right)=\pi\left\langle\phi^{*}\left(x^{*}\right) \varphi_{B}\left(a_{1} ; l_{1}\right) \cdots \varphi_{-l_{\nu}+1}^{B}\left(a_{\nu} ; l_{\nu}\right)\right. \\
\left.\cdots \varphi_{B}\left(a_{n} ; l_{n}\right)\right\rangle / \tau_{B}(L), \\
\beta_{0 \nu}\left(x^{*} ; L\right)=\frac{\pi}{2 \sin \pi l_{\nu}} \widehat{w}_{B \nu}^{*}\left(x^{*} ; L\right), \\
\alpha_{\mu \nu}(L)=2 \sin \pi l_{\mu} \tau_{B \mu \nu}^{k}\left(L ; l_{\mu},-l_{\nu}+1\right) / \tau_{B}(L), \\
\beta_{\mu \nu}(L)=-\delta_{\mu \nu}+2 \sin \pi l_{\mu \nu} \tau_{B / \nu}\left(L ; l_{\mu},-l_{\nu}\right) / \tau_{B}(L)
\end{gathered}
$$

where we have set

$$
\begin{aligned}
& \tau_{B \mu \nu}^{k}\left(L ; l, l^{\prime}\right)=\left\langle\varphi_{B}\left(a_{1} ; l_{1}\right) \cdots \varphi_{l}^{B *}\left(a_{\mu} ; l_{\mu}\right)\right. \\
& \cdots \varphi_{l^{\prime}}^{B}\left(a_{\nu} ; l_{\nu}\right) \cdots \varphi_{B}\left(a_{n} ; l_{n}\right)>\quad(\mu \neq \nu) \\
& =\left\langle\varphi_{B}\left(a_{1} ; l_{1}\right) \cdots \varphi_{B i l^{\prime}}\left(a_{\mu} ; l_{\mu}\right) \cdots \varphi_{B}\left(a_{n} ; l_{n}\right)\right\rangle \quad(\mu=\nu) \text {. }
\end{aligned}
$$

Note also that (4.3.41) implies

$$
\begin{aligned}
& \frac{\partial}{\partial\left(-m a_{\nu}^{-}\right)}-\log \tau_{B}(L)=-c_{-l_{\nu}+1}^{(\nu)}\left(\widehat{\omega}_{B \nu}\right), \\
& -\frac{\partial}{\partial\left(m a_{\nu}^{+}\right)} \log \tau_{B}(L)=-c_{-l_{\nu}+1}^{*(\nu)}\left(\widehat{\omega}_{B \nu}^{*}\right) .
\end{aligned}
$$

Formulas for the Fermi wave functions are obtained similarly. We set $w_{F}^{( \pm)}\left(x^{*}, x ; L\right)={ }^{t}\left(w_{F_{ \pm+}}\left(x^{*}, x ; L\right), w_{F^{ \pm-}}\left(x^{*}, x ; L\right)\right)$. Assuming $l_{\nu} \notin Z+\frac{1}{2} \quad(\nu=1, \cdots, n)$ we have 
$(4.4 .13)_{0} \quad w_{F}^{( \pm)}\left(x^{*}, x ; L\right)=\frac{i \tau_{F}(L)}{\pi}\left\{\begin{array}{l}\widetilde{w}_{\frac{1}{2}}^{*}\left[x^{*}\right] \\ -\widetilde{w}_{\frac{1}{2}}\left[x^{*}\right]\end{array}\right.$

$$
\begin{gathered}
+\sum_{j=0}^{\infty} c_{\frac{1}{2}+j}^{(0)}\left(w_{F}^{( \pm)}\right) \cdot w_{\frac{1}{2}+j}\left[x^{*}\right]+\sum_{j=0}^{\infty} c_{\frac{1}{2}+j}^{*(0)}\left(w_{F}^{( \pm)}\right) \cdot w_{\frac{1}{2}+j}^{*}\left[x^{*}\right], \\
\left\{\begin{array}{l}
c_{\frac{1}{2}+j}^{(0)}\left(w_{F}^{( \pm)}\right)=\left\langle: \psi_{ \pm}^{*}\left(x^{*}\right) \phi_{\frac{1}{2}+j}\left(x^{*}\right): \varphi_{F}\left(a_{1} ; l_{1}\right) \cdots \varphi_{F}\left(a_{n} ; l_{n}\right)\right\rangle(j \in \mathbb{Z}) \\
c_{\frac{1}{2}+j}^{*(0)}\left(w_{F}^{( \pm)}\right)=c_{-\frac{1}{2}-j}^{(0)}\left(w_{F}^{( \pm)}\right) \quad(j \geqq 0) .
\end{array}\right.
\end{gathered}
$$

(4. 4. 13), $\quad w_{F}^{( \pm)}\left(x^{*}, x ; L\right)=\sum_{j=1}^{\infty} c_{-l_{\nu}+j}^{(\nu)}\left(w_{F}^{( \pm)}\right) \cdot w_{-l_{\nu}+j}\left[a_{\nu}\right]$

$$
+\sum_{j=0}^{\infty} c_{l_{\nu}+j}^{*(\nu)}\left(w_{F}^{( \pm)}\right) \cdot w_{l_{\nu}+j}^{*}\left[a_{\nu}\right]
$$

$$
\left\{\begin{array}{l}
c_{-l_{\nu}+j}^{(\nu)}\left(w_{F}^{( \pm)}\right)=\left\langle\psi_{ \pm}^{*}\left(x^{*}\right) \varphi_{F}\left(a_{1} ; l_{1}\right) \cdots \varphi_{-l_{\nu}+j}^{F}\left(a_{\nu} ; l_{\nu}\right) \cdots \varphi_{F}\left(a_{n} ; l_{n}\right)\right\rangle(j \in \mathbb{Z}) \\
c_{l_{\nu}+j}^{*(\nu)}\left(w_{F}^{(+)}\right)=c_{-l_{\nu}-j}^{(\nu)}\left(w_{F}^{( \pm)}\right) \quad(j \geqq 0) .
\end{array}\right.
$$

$(4.4 .14)_{\mu \nu} \quad w_{F \mu}(x ; L)=-\frac{i \tau_{F}(L)}{2 \cos \pi l_{\mu}} \cdot \delta_{\mu \nu} w_{-l_{\nu}}\left[a_{\nu}\right]$

$$
\begin{gathered}
+\sum_{j=1}^{\infty} c_{-l_{\nu}+j}^{(\nu)}\left(w_{F^{\mu}}\right) \cdot w_{-l_{\nu}+j}\left[a_{\nu}\right]+\sum_{j=0}^{\infty} c_{l_{\nu}+j}^{*(\nu)}\left(w_{F^{\mu}}\right) \cdot w_{l_{\nu}+j}^{*}\left[a_{\nu}\right] \\
\left\{\begin{array}{cc}
c_{-l_{\nu}+j}^{(\nu)}\left(w_{F \mu}\right)= \pm\left\langle\varphi_{F}\left(a_{1} ; l_{1}\right) \cdots \varphi_{l_{\mu}}^{*}\left(a_{\mu} ; l_{\mu}\right) \cdots \varphi_{-l+j}^{F}\left(a_{\nu} ; l_{\nu}\right) \cdots \varphi_{F}\left(a_{n} ; l_{n}\right)\right\rangle \\
(\mu \lessgtr \nu, j \in \mathbb{Z}) \\
=\left\langle\varphi_{F}\left(a_{1} ; l_{1}\right) \cdots \varphi_{F l_{\nu},-l_{\nu}+j}^{*}\left(a_{\nu} ; l_{\nu}\right) \cdots \varphi_{F}\left(a_{n} ; l_{n}\right)\right. \\
(\mu=\nu, j \in \mathbb{Z}) \\
c_{l_{\nu}+j}^{*(\nu)}\left(w_{F \mu}\right)=\frac{i \tau_{F}(L)}{2 \cos \pi l_{\mu}} \delta_{j 0} \delta_{\mu \nu}+c_{-l_{\nu}-j}^{(\nu)}\left(w_{F^{\prime} \mu}\right) & (j \geqq 0) .
\end{array}\right.
\end{gathered}
$$

$(4.4 .15)_{\mu_{\nu}} \quad w_{F \mu}^{*}\left(x^{*} ; L\right)=-\frac{i \tau_{F}(L)}{2 \cos \pi l_{\mu}} \cdot \delta_{\mu \nu} w_{-l_{\nu}}^{*}\left[a_{\nu}\right]$

$$
\begin{gathered}
+\sum_{j=0}^{\infty} c_{l_{\nu}+j}^{(\nu)}\left(w_{F^{\mu}}^{*}\right) \cdot w_{l_{\nu}+j}\left[a_{\nu}\right]+\sum_{j=1}^{\infty} c_{-l_{\nu}+j}^{*(\nu)}\left(w_{F^{\prime} \mu}^{*}\right) \cdot w_{-l_{\nu}+j}^{*}\left[a_{\nu}\right], \\
\left\{\begin{array}{rr}
c_{l_{\nu}+j}^{(\nu)}\left(w_{F^{\prime}}^{*}\right)=\frac{i \tau_{F}(L)}{2 \cos \pi l_{\mu}} \delta_{j 0} \delta_{\mu \nu}+c_{-l_{\nu}-j}^{*(\nu)}\left(w_{F^{\mu}}^{*}\right) \quad(j \geqq 0) \\
c_{-l_{\nu}+j}^{*(\nu)}\left(w_{F \mu}^{*}\right)=\mp\left\langle\varphi _ { F } ( a _ { 1 } ; l _ { 1 } ) \cdots \varphi _ { - l _ { \mu } } ^ { F } ( a _ { \mu } ; l _ { \mu } ) \cdots \varphi _ { l _ { \nu } - j } ^ { F } ( a _ { \nu } ^ { * } ; l _ { \nu } ) \cdots \varphi _ { F } \left( a_{n} ;\right.\right. \\
(\mu \lessgtr \nu, j \in \mathbb{Z}) \\
=-\left\langle\varphi_{F}\left(a_{1} ; l_{1}\right) \cdots \varphi_{F-l_{\mu}, l_{\mu}-j}^{*}\left(a_{\mu} ; l_{\mu}\right) \cdots \varphi_{F}\left(a_{n} ; l_{n}\right)\right\rangle \\
(\mu=\nu, j \in \mathbb{Z})
\end{array}\right.
\end{gathered}
$$

where $w_{l}^{\prime}[a]=w_{l}\left(-\left(x^{*}-a\right)^{-}+i 0,\left(x^{*}-a\right)^{+}-i 0\right)$, etc. 
We set

$$
\begin{aligned}
& \widehat{\mho}_{F}\left(x^{*}, x ; L\right)=\pi i w_{F}\left(x^{*}, x ; L\right) / \tau_{F}(L), \\
& \widehat{w}_{F^{\prime \prime}}(x ; L)=2 i \cos \pi l_{\mu} \cdot w_{F^{\mu}}(x ; L) / \tau_{F}(L), \\
& \widehat{\mho}_{F^{\prime}}^{*}\left(x^{*} ; L\right)=2 i \cos \pi l_{\mu} \cdot w_{F^{\mu}}^{*}\left(x^{*} ; L\right) / \tau_{F}(L) .
\end{aligned}
$$

Proposition 4. 4.2. For $l_{1}, \cdots, l_{n} \in \mathbb{C}-\left(\mathbb{Z}+\frac{1}{2}\right)$, the Euclidean continuations of $\widehat{w}_{F}^{(t)}\left(x^{*}, x ; L\right), \widehat{w}_{F \mu}(x ; L)(1 \leqq \mu \leqq n)$ and $\widehat{w}_{F \mu}^{*}\left(x^{*} ; L\right)$ $(1 \leqq \mu \leqq n)$ provide the canonical independent elements of $W_{F^{*}, z^{*}, a_{1}, \ldots, a_{n}}^{\left( \pm 0, l_{n}\right.}$ and $W_{F}^{*}, l_{1}, \cdots, \cdots, l_{n}$, strict respectively. If $k-1 / 2<l_{1}, \cdots, l_{n}<k+1 / 2$ for some $k \in \mathbb{Z}, \hat{\omega}_{F}^{(+)}\left(z^{*}, z ; L\right), \hat{w}_{F_{\mu}}(z ; L)$ and $\hat{w}_{F_{\mu}}^{*}\left(z^{*} ; L\right)$ coincide with those $w_{0}^{( \pm)}\left(z^{*}, z ; L\right), w_{\mu}(z ; L)$ and $w_{\mu}^{*}\left(z^{*} ; L\right)$ in Chapter III respectively.

(4. 4.13) $)_{0} \sim(4.4 .15)_{\mu \nu}$ and (4.3.73) now yield the following (cf. (3. 2.22)).

$$
\text { (4.4.17) } \begin{aligned}
\alpha_{0 \nu}^{( \pm)}\left(L+\frac{1}{2}\right) & =\pi i\left\langle\psi_{ \pm}^{*}\left(x^{*}\right) \varphi_{F}\left(a_{1} ; l_{1}\right) \cdots \varphi_{-l_{\nu}+1}^{F}\left(a_{\nu} ; l_{\nu}\right)\right. \\
\left.\cdots \varphi_{F}\left(a_{n} ; l_{n}\right)\right\rangle / \tau_{F}(L) & \\
\beta_{0 \nu}^{( \pm)}\left(L+\frac{1}{2}\right) & =\frac{\pi}{2 \cos \pi l_{\nu}} \widehat{\vartheta}_{F^{*} \pm}^{*}\left(x^{*} ; L\right) . \\
\alpha_{\mu \nu}\left(L+\frac{1}{2}\right) & =2 i \cos \pi l_{\mu} \cdot \tau_{F \mu \nu}^{*}\left(L ; l_{\mu},-l_{\nu}+1\right) / \tau_{F}(L) \\
\beta_{\mu \nu}\left(L+\frac{1}{2}\right) & =-\delta_{\mu \nu}+2 i \cos \pi l_{\nu} \cdot \tau_{F \mu \nu}^{*}\left(L ; l_{\mu},-l_{\nu}\right) / \tau_{F}(L)
\end{aligned}
$$

where

$$
\begin{aligned}
& \tau_{F^{*}{ }^{*} \nu}\left(L ; l, l^{\prime}\right)= \pm\left\langle\varphi_{F}\left(a_{1} ; l_{1}\right) \cdots \varphi_{l}^{F^{*}}\left(a_{\mu} ; l_{\mu}\right) \cdots \varphi_{l^{\prime}}^{F}\left(a_{\nu} ; l_{\nu}\right)\right. \\
&\left.\cdots \varphi_{F}\left(a_{n} ; l_{n}\right)\right\rangle(\mu \lessgtr \nu) \\
&=\left\langle\varphi_{F}\left(a_{1} ; l_{1}\right) \cdots \varphi_{F^{*} l^{\prime}}\left(a_{\mu} ; l_{\mu}\right) \cdots \varphi_{F}\left(a_{n} ; l_{n}\right)\right\rangle \quad(\mu=\nu) .
\end{aligned}
$$

$$
\begin{aligned}
& \frac{\partial}{\partial\left(-m a_{\nu}^{-}\right)} \log \tau_{F}(L)=c_{-l_{\nu}+1}^{(\nu)}\left(\widehat{\boldsymbol{v}}_{F_{\nu}}\right) \\
& \frac{\partial}{\partial\left(m a_{\nu}^{+}\right)} \log \tau_{F}(L)=c_{-l_{\nu}+1}^{*(\nu)}\left(\widehat{\iota}_{F_{\nu}}^{*}\right) .
\end{aligned}
$$

Notice that (4. 4.11) and (4.4.17) give different expressions for the same 
quantities $\alpha(L), \beta(L)$. More generally by virtue of the isomorphism (3.2.15) we have the following relations between the Bose and Fermi wave functions:

$$
\begin{aligned}
& \left\langle\phi^{*}\left(x^{*}\right) \varphi_{B}\left(a_{1} ; l_{1}+\frac{1}{2}\right) \cdots \varphi_{B}\left(a_{n} ; l_{n}+\frac{1}{2}\right) \phi(x)\right\rangle \mid \tau_{B}\left(L+\frac{1}{2}\right) \\
& =i\left\langle\phi_{-}^{*}\left(x^{*}\right) \varphi_{F}\left(a_{1} ; l_{1}\right) \cdots \varphi_{F}\left(a_{n} ; l_{n}\right) \phi_{+}(x)\right\rangle / \tau_{F}(L),
\end{aligned}
$$

(4. 4.21) $\left\langle\varphi_{B}\left(a_{1} ; l_{1}+\frac{1}{2}\right) \cdots \varphi_{l_{\mu+\frac{1}{2}}}^{B *}\left(a_{\mu} ; l_{\mu}+\frac{1}{2}\right)\right.$

$$
\begin{gathered}
\left.\cdots \varphi_{B}\left(a_{n} ; l_{n}+\frac{1}{2}\right) \phi(x)\right\rangle / \tau_{B}\left(L+\frac{1}{2}\right) \\
=i\left\langle\varphi_{F}\left(a_{1} ; l_{1}\right) \cdots \varphi_{l_{\mu}}^{F *}\left(a_{\mu} ; l_{\mu}\right) \cdots \varphi_{F}\left(a_{n} ; l_{n}\right) \psi_{+}(x)\right\rangle / \tau_{F}(L),
\end{gathered}
$$

(4. 4. 22) $\left\langle\phi^{*}\left(x^{*}\right) \varphi_{B}\left(a_{1} ; l_{1}+\frac{1}{2}\right) \cdots \varphi_{-l_{\nu}-\frac{1}{2}}^{B}\left(a_{\nu} ; l_{\nu}+\frac{1}{2}\right)\right.$

$$
\begin{gathered}
\left.\cdots \varphi_{B}\left(a_{n} ; l_{n}+\frac{1}{2}\right)\right\rangle \mid \tau_{B}\left(L+\frac{1}{2}\right) \\
=i\left\langle\psi_{-}^{*}\left(x^{*}\right) \varphi_{F}\left(a_{1} ; l_{1}\right) \cdots \varphi_{-l_{\nu}}^{F}\left(a_{\nu} ; l_{\nu}\right) \cdots \varphi_{B}\left(a_{n} ; l_{n}\right)\right\rangle / \tau_{B}(L) .
\end{gathered}
$$

The connection with the deformation theory enables us to express the $\tau$ functions in a closed form in terms of solutions of the non-linear total differential equations (3.3.24). Namely from (4.4.11) and (4.4.17) we have

$$
-d \log \tau_{B}\left(L+\frac{1}{2}\right)=d \log \tau_{F}(L)=\omega
$$

where $\omega=\omega(L)$ denotes the 1-form (3. 3.57), corresponding to $\boldsymbol{w}=\boldsymbol{w}(L)$. As we shall see in Section 4.5 , the $\tau$-functions satisfy the boundary conditions $\tau_{B}(L), \tau_{F}(L) \rightarrow 1$ as $\left|a_{\mu}-a_{\nu}\right| \rightarrow \infty(\mu \neq \nu)$. Hence (4.4.23) implies further that

$$
\tau_{B}\left(L+\frac{1}{2}\right) \cdot \tau_{F}(L)=1
$$

Remark. The relation (4.4.24) is intuitively an obvious consequence of the formulas (1.5.7) and (A.31). Observe first that the symplectic vector space $W_{B}$ in Section 4.1 and the orthogonal $W_{F}$ are the same 
objects in the $u$-representation, including the holonomic decomposition $V^{*} \oplus V$. Moreover the rotation $T$ induced by $\varphi_{B}=\varphi_{B}\left(a ; l+\frac{1}{2}\right)$ is identical with the one induced by $\varphi_{F}=\varphi_{F}(a ; l)$. If we denote by $(1 \pm E) / 2$ the projection operators onto $V^{*}$ or $V,(1.5 .7)$ and (A.31) yield

$$
\begin{aligned}
& \left\langle\varphi_{B}\right\rangle^{2}=\operatorname{nr}\left(\varphi_{B}\right) \operatorname{det}\left(\frac{1+T}{2}+E^{-1} \frac{1-T}{2}\right)^{-1} \\
& \left\langle\varphi_{F}\right\rangle^{2}=\operatorname{nr}\left(\varphi_{F}\right) \operatorname{det}\left(\frac{1+T}{2}+E \frac{1-T}{2}\right) .
\end{aligned}
$$

Noting $\left\langle\varphi_{B}\right\rangle=1,\left\langle\varphi_{F}\right\rangle=1$ and $E^{2}=1$ we see that $\operatorname{nr}\left(\varphi_{B}\right) \cdot \operatorname{nr}\left(\varphi_{F}\right)=1$. Hence (4. 4.24) applied to the products $\varphi_{B}\left(a_{1} ; l_{1}+\frac{1}{2}\right) \cdots \varphi_{B}\left(a_{n} ; l_{n}+\frac{1}{2}\right)$ and $\varphi_{F}\left(a_{1} ; l_{1}\right) \cdots \varphi_{F}\left(a_{n} ; l_{n}\right)$ shows that their expectation values are mutually inverse.

We shall now generalize the above discussion to include $n(n-1) / 2$ parameters 1 . As shown in I (or in Appendix to this Chapter), in investigating the vacuum expectation values of the form $\left\langle g_{1} \cdots g_{n}\right\rangle$, it is natural to consider an extended sympletic or orthogonal space parametrized by a symmetric matrix $\Lambda=\left(\lambda_{\mu \nu}\right)$ with $\lambda_{\nu \nu}=1(\nu=1, \cdots, n)$. Let $e_{1}, \cdots, e_{n}$ be a basis of $\boldsymbol{C}^{n}$. We equip it with a symmetric (possibly degenerate) inner product by setting $\left\langle e_{\mu}, e_{\nu}\right\rangle=\lambda_{\mu \nu}(\mu, \nu=1, \cdots, n)$. Consider the sympletic vector space $W_{B}(\Lambda)=\left(W_{B} \otimes C^{2}\right) \otimes C^{a}$, where the inner product $\langle,\rangle_{A}$ and the expectation value \langle\rangle$_{A}$ among the ideal basis $\phi^{(\mu)}(u)$ $=\phi(u) \otimes e_{\mu}, \phi^{*(u)}(u)=\phi^{*}(u) \otimes e_{\mu}$ are given as follows.

$$
\begin{gathered}
\left(\begin{array}{cc}
\left\langle\phi^{(\mu)}(u), \phi^{(\nu)}\left(u^{\prime}\right)\right\rangle_{A} & \left\langle\phi^{(\mu)}(u), \phi^{*(\nu)}\left(u^{\prime}\right)\right\rangle_{A} \\
\left\langle\phi^{*(\mu)}(u), \phi^{(\nu)}\left(u^{\prime}\right)\right\rangle_{A} & \left\langle\phi^{*(\mu)}(u), \phi^{*(\nu)}\left(u^{\prime}\right)\right\rangle_{A^{\prime}}
\end{array}\right) \\
=\lambda_{\mu \nu}\left(\begin{array}{c}
1 \\
1
\end{array}\right) 2 \pi u \delta\left(u+u^{\prime}\right), \\
\left(\begin{array}{cc}
\left\langle\phi^{(\mu)}(u) \phi^{(\nu)}\left(u^{\prime}\right)\right\rangle_{A} & \left\langle\phi^{(\mu)}(u) \phi^{*(\nu)}\left(u^{\prime}\right)\right\rangle_{A} \\
\left\langle\phi^{*(\mu)}(u) \phi^{(\nu)}\left(u^{\prime}\right)\right\rangle_{A} & \left\langle\phi^{*(\mu)}(u) \phi^{*(\nu)}\left(u^{\prime}\right)\right\rangle_{A}
\end{array}\right) \\
=\lambda_{\mu \nu}\left(\begin{array}{c}
1 \\
1
\end{array}\right) 2 \pi u_{+} \delta\left(u+u^{\prime}\right) .
\end{gathered}
$$

We define operators $\phi_{l}^{(\nu)}(x), \phi_{l}^{*(\nu)}(x)((4.3 .5)-(4.3 .6)), \varphi_{B}^{(\nu)}(a ; l)$, $\varphi_{l^{\prime}}^{B(\nu)}(a ; l), \varphi_{l^{\prime}}^{B^{*}(\nu)}(a ; l)((4.3 .11)-(4.3 .13))$ and $\varphi_{B}^{(\nu)} l_{1} l_{2}(a ; l), \varphi_{B}^{(\nu) l_{1} l_{2}}(a ; l)$, $\varphi_{B}^{(\nu)} l_{1} i_{2}(a ; l)((4.3 .30)-(4.3 .32))$ by the formulas cited here, using $\phi^{(\nu)}(u)$, 
$\phi^{*(\nu)}(u)$ in place of $\phi(u), \phi^{*}(u)$ respectively. From the definition (4. 4. 25) and the condition $\lambda_{\nu \nu}=1$, it follows in particular that the expectation values (and hence the inner product) of $\phi^{(\mu)}(u)-\lambda_{\mu \nu} \phi^{(\nu)}(u)$ and $\phi^{(\nu)}\left(u^{\prime}\right), \phi^{*(\nu)}\left(u^{\prime}\right)$ vanish identically:

$$
\begin{aligned}
& \left\langle\left(\phi^{(\mu)}(u)-\lambda_{\mu \nu} \phi^{(\nu)}(u)\right) \phi^{(\nu)}\left(u^{\prime}\right)\right\rangle_{A}=0, \\
& \left\langle\left(\phi^{(\mu)}(u)-\lambda_{\mu \nu} \phi^{(\nu)}(u)\right) \phi^{*(\nu)}\left(u^{\prime}\right)\right\rangle_{A}=0, \\
& \left\langle\phi^{(\nu)}\left(u^{\prime}\right)\left(\phi^{(\mu)}(u)-\lambda_{\mu \nu} \phi^{(\nu)}(u)\right)\right\rangle_{A}=0, \\
& \left\langle\phi^{*(\nu)}\left(u^{\prime}\right)\left(\phi^{(\mu)}(u)-\lambda_{\mu \nu} \phi^{(\nu)}(u)\right)\right\rangle_{A}=0,
\end{aligned}
$$

and similarly for $\phi^{*(\mu)}(u)-\lambda_{\mu \nu} \phi^{*(\nu)}(u)$. This observation leads us to the following consequences: (i) the local expansion formulas (4.3.26)(4. 3. 27), (4. 3. 33)-(4.3.36) are valid for the products $\phi^{(\nu)}(x) \varphi_{B}^{(\nu)}(a ; l)$, etc. having the same superfix (accordingly the right hand sides of the formulas should be superfixed by the same $\nu$ ), while (ii) products $\left(\phi^{(\mu)}(x)-\lambda_{\mu \nu} \phi^{(\nu)}(x)\right) \varphi_{B}^{(\nu)}(a ; l)$, etc. with different $\mu, \nu$ cause no singularities at $x=a$. To see (ii) we note, for instance,

$$
\begin{aligned}
\operatorname{Nr}\left(\left(\phi^{(\mu)}\right.\right. & \left.\left.(x)-\lambda_{\mu \nu} \phi^{(\nu)}(x)\right) \varphi_{B}^{(\nu)}(a ; l)\right) \\
& =\left(\phi^{(\mu)}(x)-\lambda_{\mu \nu} \phi^{(\nu)}(x)\right) \cdot e^{\rho_{B}(\nu)(a ; l) / 2} \\
& =\sum_{j=-\infty}^{+\infty}\left(\phi_{j}^{(\mu)}(x)-\lambda_{\mu \nu} \phi_{j}^{(\nu)}(x)\right) \cdot e^{\rho_{B}(\nu)(a ; l) / 2} \cdot v_{j}[a],
\end{aligned}
$$

and so forth.

Thus the commutation relations among the $\varphi$-fields and the free fields $\phi^{(\mu)}(x), \phi^{*(\mu)}(x)$ read as follows. For convenience we use the vector notation $\vec{\phi}(x)=\left(\phi^{(1)}(x), \cdots, \phi^{(n)}(x)\right), \vec{\phi}^{*}(x)=\left(\phi^{*(1)}(x), \cdots, \phi^{*(n)}(x)\right)$.

$$
\begin{aligned}
& \text { (4. 4.27) } \varphi_{B}^{(\nu)}(a ; l) \vec{\phi}(x) \\
& =\left\{\begin{array}{lll}
\vec{\phi}(x) \varphi_{B}^{(\nu)}(a ; l) & \text { if } & x^{+}>a^{+} \text {and } x^{-}<a^{-} \\
\vec{\phi}(x) \varphi_{B}^{(\nu)}(a ; l) M_{\nu}(l) & \text { if } \quad x^{+}<a^{+} \text {and } x^{-}>a^{-},
\end{array}\right. \\
& \varphi_{B}^{(\nu)}(a ; l) \vec{\phi}^{*}(x) \\
& =\left\{\begin{array}{l}
\vec{\phi}^{*}(x) \varphi_{B}^{(\nu)}(a ; l) \quad \text { if } \quad x^{+}>a^{+} \text {and } x^{-}<a^{-} \\
\vec{\phi}^{*}(x) \varphi_{B}^{(\nu)}(a ; l) M_{\nu}(l)^{-1} \quad \text { if } \quad x^{+}<a^{+} \text {and } x^{-}>a^{-}
\end{array}\right.
\end{aligned}
$$

Here we have set 


$$
\begin{aligned}
& M_{\nu}(l)^{ \pm 1}=1+\left(e^{ \pm 2 \pi i l}-1\right) E_{\nu} \Lambda
\end{aligned}
$$

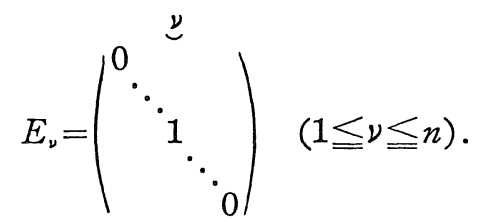

Likewise $\varphi_{-l+j}^{B(\nu)}(a ; l)$ and $\varphi_{l+j}^{B^{*}(\nu)}(a ; l) \quad(l \in \mathbb{Z})$ satisfy (4.4.27), where $\varphi_{B}^{(\nu)}(a ; l)$ are replaced by $\varphi_{-l+j}^{B(\nu)}(a ; l)$ and $\varphi_{l+j}^{B^{*}(\nu)}(a ; l)$ respectively.

We now consider the $n \times n$ matrices $w_{B}\left(x^{*}, x ; L, \Lambda\right), w_{B}(x ; L, \lambda)$ and $w_{B}^{*}\left(x^{*} ; L, \Lambda\right)$ of wave functions, whose $(\mu, \nu)$-th components are given by

$$
\begin{aligned}
& w_{B}\left(x^{*}, x ; L, \Lambda\right)_{\mu \nu}=\left\langle\phi^{*(\mu)}\left(x^{*}\right) \varphi_{B}^{(1)}\left(a_{1} ; l_{1}\right) \cdots \varphi_{B}^{(n)}\left(a_{n} ; l_{n}\right) \phi^{(\nu)}(x)\right\rangle_{A}, \\
& w_{B}(x ; L, \Lambda)_{\mu \nu}=\left\langle\varphi_{B}^{(1)}\left(a_{1} ; l_{1}\right) \cdots \varphi_{l_{\mu}^{*}}^{B^{*}(\mu)}\left(a_{\mu} ; l_{\mu}\right) \cdots \varphi_{B}^{(n)}\left(a_{n} ; l_{n}\right) \phi^{(\nu)}(x)\right\rangle_{\Lambda}, \\
& w_{B}^{*}\left(x^{*} ; L, \Lambda\right)_{\mu \nu}=\left\langle\phi^{*(\mu)}\left(x^{*}\right) \varphi_{B}^{(1)}\left(a_{1} ; l_{1}\right) \cdots \varphi_{-l_{\nu}}^{B(\nu)}\left(a_{\nu} ; l_{\nu}\right) \cdots \varphi_{B}^{(n)}\left(a_{n} ; l_{n}\right)\right\rangle_{A} .
\end{aligned}
$$

We set also

$$
\text { (4. 4. 30) } \tau_{B}(L, \Lambda)=\tau_{B}\left(a_{1}, \cdots, a_{n} ; L, \Lambda\right)=\left\langle\varphi_{B}^{(1)}\left(a_{1} ; l_{1}\right) \cdots \varphi_{B}^{(n)}\left(a_{n} ; l_{n}\right)\right\rangle_{A} \text {. }
$$

From (4. 4. 4) ${ }_{0} \sim(4.4 \text {. 5) })_{\mu \nu}^{*}$ we may readily write down the local expansions for (4. 4. 29). Assuming $l_{1}, \cdots, l_{n} \notin \mathbb{Z}$ we have

$$
\begin{aligned}
& \text { (4. 4. 31) })_{0} w_{B}\left(x^{*}, x ; L, \Lambda\right) \equiv \frac{1}{\pi} \tau_{B}(L, \Lambda) \cdot A \cdot \widetilde{v}_{0}\left[x^{*}\right] \\
& \text { (4.4.31), } w_{B}\left(x^{*}, x ; L, \Lambda\right) \equiv \sum_{j=1}^{\infty} \widetilde{C}_{j, \Lambda}\left(w_{B}\right) E_{\nu} \Lambda \cdot v_{-l_{\nu}+j}\left[a_{\nu}\right] \\
& +\sum_{j=0}^{\infty} \widetilde{C}_{j, \Lambda}^{*}\left(w_{B}\right) E_{\nu} \Lambda \cdot v_{l_{\nu}+j}^{*}\left[a_{\nu}\right]
\end{aligned}
$$

where $\equiv$ signifies "modulo single-valued regular functions", and $\widetilde{C}_{j, A}\left(w_{B}\right)$, $\widetilde{C}_{j, A}^{*}\left(w_{B}\right)$ denote matrices with entries

$$
\left\{\begin{array}{l}
\widetilde{C}_{j, A}\left(w_{B}\right)_{\mu \nu}=\left\langle\phi^{*(\mu)}\left(x^{*}\right) \varphi_{B}^{(1)}\left(a_{1} ; l_{1}\right) \cdots \varphi_{-\nu_{\nu}+j}^{B(\nu)}\left(a_{\nu} ; l_{\nu}\right) \cdots \varphi_{B}^{(n)}\left(a_{n} ; l_{n}\right)\right\rangle_{A} \\
(j \in \mathbb{Z}) \\
\widetilde{C}_{j, A}^{*}\left(w_{B}\right)=\widetilde{C}_{-j, \Lambda}\left(w_{B}\right) \quad(j \geqq 0) .
\end{array}\right.
$$

Similarly we have

$$
\text { (4. 4. 32) }{ }_{\nu} \quad w_{B}(x ; L, \Lambda) \equiv \frac{\tau_{B}(L ; \Lambda)}{2 \sin \pi l_{\nu}} E_{\nu} \Lambda \cdot v_{-l_{\nu}}\left[a_{\nu}\right]
$$




$$
\begin{gathered}
+\sum_{j=1}^{\infty} C_{j, \Lambda}\left(w_{B}\right) E_{\nu} \Lambda \cdot v_{-l_{\nu}+j}\left[a_{\nu}\right]+\sum_{j=0}^{\infty} C_{j, A}^{*}\left(w_{B}\right) E_{\nu} \Lambda \cdot v_{l_{\nu}+j}^{*}\left[a_{\nu}\right] \\
\left\{\begin{array}{cc}
C_{j, A}\left(w_{B}\right)_{\mu \nu}=\left\langle\varphi_{B}^{(1)}\left(a_{1} ; l_{1}\right) \cdots \varphi_{l_{\mu}}^{B *(\mu)}\left(a_{\mu} ; l_{\mu}\right) \cdots \varphi_{-l_{\nu}+j}^{B(\nu)}\left(a_{\nu} ; l_{\nu}\right) \cdots \varphi_{B}^{(n)}\left(a_{n} ; l_{n}\right)\right\rangle_{A} \\
(\mu \neq \nu, j \in \mathbb{Z}) \\
=\left\langle\varphi_{B}^{(1)}\left(a_{1} ; l_{1}\right) \cdots \varphi_{B}^{(\nu) *} l_{\nu,-l_{\nu}+j}\left(a_{\nu} ; l_{\nu}\right) \cdots \varphi_{B}^{(n)}\left(a_{n} ; l_{n}\right)\right\rangle_{A} \\
(\mu=\nu, j \in \mathbb{Z}) \\
C_{j, \Lambda}^{*}\left(w_{B}\right)=-\frac{\tau_{B}(L ; \Lambda)}{2 \sin \pi L} \delta_{j 0}+C_{-j, A}\left(w_{B}\right) & (j \geqq 0)
\end{array}\right.
\end{gathered}
$$

(4. 4. 32) ${ }_{\nu}^{*} \quad{ }^{t} w_{B}^{*}\left(x^{*} ; L, \Lambda\right) \equiv \sum_{j=0}^{\infty} C_{j, \Lambda}\left(w_{B}^{*}\right) E_{\nu} \Lambda \cdot v_{l_{\nu}+j}^{\prime}\left[a_{\nu}\right]$

$$
\begin{gathered}
+\frac{\tau_{B}(L ; \Lambda)}{2 \sin \pi l_{\nu}} E_{\nu} \Lambda \cdot v_{-l_{\nu}}^{* \prime}\left[a_{\nu}\right]+\sum_{j=1}^{\infty} C_{j, A}^{*}\left(w_{B}^{*}\right) E_{\nu} \Lambda \cdot v_{-l_{\nu}+j}^{* \prime}\left[a_{\nu}\right] \\
\left\{\begin{array}{c}
C_{j, A}\left(w_{B}^{*}\right)=-\frac{\tau_{B}(L ; \Lambda)}{2 \sin \pi L} \delta_{j 0}+C_{-j, \Lambda}^{*}\left(w_{B}^{*}\right) \quad(j \geqq 0) \\
C_{j, A}^{*}\left(w_{B}^{*}\right)_{\mu \nu}=\left\langle\varphi_{B}^{(1)}\left(a_{1} ; l_{1}\right) \cdots \varphi_{l_{\mu}-j}^{B *(\mu)}\left(a_{\mu} ; l_{u}\right) \cdots \varphi_{-l_{\nu}}^{B(\nu)}\left(a_{\nu} ; l_{\nu}\right) \cdots \varphi_{B}^{(n)}\left(a_{n} ; l_{n}\right)\right\rangle_{1} \\
(\mu \neq \nu, j \in \mathbb{Z}) \\
=\left\langle\varphi_{B}^{(1)}\left(a_{1} ; l_{1}\right) \cdots \varphi_{B}^{(\nu) *} l_{\nu-j,-l_{\nu}}\left(a_{\nu} ; l_{\nu}\right) \cdots \varphi_{B}^{(n)}\left(a_{n} ; l_{n}\right)\right\rangle_{A} \\
(\mu=\nu, j \in \mathbb{Z}) .
\end{array}\right.
\end{gathered}
$$

Set

$$
\begin{aligned}
& \widehat{w}_{B}\left(x^{*}, x ; L, \Lambda\right)=\pi w_{B}\left(x^{*}, x ; L, \Lambda\right) / \tau_{B}(L, \Lambda), \\
& \widehat{w}_{B}(x ; L, \Lambda)=2 \sin \pi L \cdot w_{B}(x ; L, \Lambda) / \tau_{B}(L, \Lambda), \\
& { }^{t} \widehat{w}_{B}^{*}\left(x^{*} ; L, \Lambda\right)=2 \sin \pi L \cdot{ }^{t} w_{B}^{*}\left(x^{*} ; L, \Lambda\right) / \tau_{B}(L, \Lambda),
\end{aligned}
$$

and denote by $\widehat{w}_{B \mu}\left(x^{*}, x ; L, \Lambda\right), \widehat{w}_{B \mu}(x ; L, \Lambda)$ and ${ }^{t} \widehat{w}_{B \mu}^{*}\left(x^{*} ; L, \Lambda\right)$ the corresponding $\mu$-th row vectors of (4.4.33).

Proposition 4. 4. 3. For $l_{1}, \cdots, l_{n} \in \boldsymbol{C}-\mathbb{Z}$, the Euclidean continuations $\widehat{w}_{B \nu}\left(z^{*}, z ; L, \Lambda\right), \widehat{w}_{B \mu}(z ; L, \Lambda)(1 \leqq \mu \leqq n)$ and $\widehat{\boldsymbol{w}}_{B \mu}^{*}\left(z^{*} ; L, \Lambda\right)$ provide canonical independent elements of $W_{B, 2^{*}, \cdots, a_{1}, \cdots, l_{n}, l_{n}, \text { strict }}^{0}\left(\Lambda, \lambda_{(\nu)}\right) \quad\left(\lambda_{(\nu)}\right.$ $=\left(\lambda_{1 \nu}, \cdots, \lambda_{n \nu}\right)=\nu$-th row of $\left.\Lambda\right)$ and of $W_{B, a_{1}, \cdots, a_{n}}^{* l_{1}, \cdots, l_{n}, \text { strict }}(\Lambda)$ respectively. If $k<l_{1}, \cdots, l_{n}<k+1$ for some $k \in \mathbb{Z}$ they coincide with $v_{0}(L ; \Lambda)$, $v_{\nu}(L ; \Lambda)$ and ${ }^{\bar{t} v_{0}(L, \Lambda)}$ respectively.

In particular we obtain (see (3.2.41)) 
(4. 4. 34) $\quad \alpha_{0 \nu}\left(L, \Lambda, \lambda_{\mu}\right)=\pi\left\langle\phi^{*(\mu)}\left(x^{*}\right) \varphi_{B}^{(1)}\left(a_{1} ; l_{1}\right) \cdots \varphi_{-l_{\nu}+1}^{B(\nu)}\left(a_{\nu} ; l_{\nu}\right) \cdots\right.$

$$
\begin{gathered}
\left.\cdots \varphi_{B}^{(n)}\left(a_{n} ; l_{n}\right)\right\rangle / \tau_{B}(L, \Lambda), \\
\beta_{0 \nu}\left(L, \Lambda, \lambda_{\mu}\right)=\frac{\pi}{2 \sin \pi l_{\nu}} \widehat{\mho}_{B}^{*}\left(x^{*} ; L, \Lambda\right)_{\mu \nu}, \\
\alpha_{\mu \nu}(L, \Lambda)=2 \sin \pi l_{\mu} \cdot \tau_{B \mu \nu}^{*}\left(L, \Lambda ; l_{\mu},-l_{\nu}+1\right) / \tau_{B}(L, \Lambda), \\
\beta_{\mu \nu}(L, \Lambda)=-\delta_{\mu \nu}+2 \sin \pi l_{\mu} \cdot \tau_{B \mu \nu}^{k}\left(L, \Lambda ; l_{\mu},-l_{\nu}\right) / \tau_{B}(L, \Lambda),
\end{gathered}
$$

where

(4. 4. 35) $\quad \tau_{B \mu \nu}^{*}\left(L, \Lambda ; l, l^{\prime}\right)=\left\langle\varphi_{B}^{(1)}\left(a_{1} ; l_{1}\right) \cdots \varphi_{l}^{B^{*}(\mu)}\left(a_{\mu} ; l_{\mu}\right) \cdots\right.$

$$
\begin{array}{r}
\left.\cdots \varphi_{l^{\prime}}^{B(\nu)}\left(a_{\nu} ; l_{\nu}\right) \cdots \varphi_{B}^{(n)}\left(a_{n} ; l_{n}\right)\right\rangle_{\Lambda}(\mu \neq \nu), \\
=\left\langle\varphi^{(1)}\left(a_{1} ; l_{1}\right) \cdots \varphi_{B i l^{\prime}}^{(\mu)}\left(a_{\mu} ; l_{\mu}\right) \cdots \varphi_{B}^{(n)}\left(a_{n} ; l_{n}\right)\right\rangle_{A} \\
(\mu=\nu) .
\end{array}
$$

(4. 4. 36)

$$
\begin{aligned}
& \frac{\partial}{\partial\left(-m a_{\nu}^{-}\right)} \log \tau_{B}(L, \Lambda)=-c_{1, A}\left(\widehat{\boldsymbol{w}}_{B}\right)_{\nu \nu}, \\
& \frac{\partial}{\partial\left(m a_{\nu}^{+}\right)} \log \tau_{B}(L, \Lambda)=-c_{1, A}^{*}\left(\widehat{\boldsymbol{w}}_{B}^{*}\right)_{\nu \nu} .
\end{aligned}
$$

The orthogonal case is quite parallel. We set

$$
\begin{aligned}
& \text { (4. 4. 37) }\left(\begin{array}{ll}
\left\langle\psi^{(\mu)}(u), \psi^{(\nu)}\left(u^{\prime}\right)\right\rangle_{\Lambda} & \left\langle\psi^{(\mu)}(u), \psi^{*(\nu)}\left(u^{\prime}\right)\right\rangle_{\Lambda} \\
\left\langle\psi^{*(\mu)}(u), \psi^{(\nu)}\left(u^{\prime}\right)\right\rangle_{\Lambda} & \left\langle\psi^{*(\mu)}(u), \psi^{*(\nu)}\left(u^{\prime}\right)\right\rangle_{A}
\end{array}\right) \\
& =\lambda_{\mu \nu}\left(\begin{array}{l}
1 \\
1
\end{array} \quad 2 \pi|u| \delta\left(u+u^{\prime}\right)\right. \\
& \left(\begin{array}{ll}
\left\langle\psi^{(\mu)}(u) \psi^{(\nu)}\left(u^{\prime}\right)\right\rangle_{A} & \left\langle\psi^{(\mu)}(u) \phi^{*(\nu)}\left(u^{\prime}\right)\right\rangle_{A} \\
\left\langle\psi^{*(\mu)}(u) \psi^{(\nu)}\left(u^{\prime}\right)\right\rangle_{A} & \left\langle\psi^{*(\mu)}(u) \psi^{*(\nu)}\left(u^{\prime}\right)\right\rangle_{A}
\end{array}\right) \\
& =\lambda_{\mu \nu}\left(\begin{array}{l}
1 \\
1
\end{array} \quad 2 \pi u_{+} \hat{o}\left(u+u^{\prime}\right)\right.
\end{aligned}
$$

and define $\varphi_{F}^{(\nu)}(a ; l)$, etc., similarly as in the symplectic case. The Fermi wave functions $w_{F}\left(x^{*}, x ; L, \Lambda\right)=\left(w_{F \varepsilon^{*} \varepsilon}\left(x^{*}, x ; L, \Lambda\right)\right)_{\varepsilon, \varepsilon^{*}= \pm}, \quad w_{F}(x ; L)$ $={ }^{t}\left({ }^{t} w_{F_{\top}}(x ; L),{ }^{t} w_{F_{-}}(x ; L)\right), \quad w_{F^{*}}^{*}\left(x^{*} ; L\right)=\left(w_{F_{+}}^{*}\left(x^{*} ; L\right), w_{F_{-}}^{*}\left(x^{*}, L\right)\right)$ are now $2 n \times 2 n, 2 n \times n$ and $n \times 2 n$ matrices, given by (4. 4. 38) $w_{F \varepsilon^{\star} \varepsilon}\left(x^{*}, x ; L, \Lambda\right)_{\mu \nu}=\left\langle\psi_{\varepsilon^{*}}^{*(\mu)}\left(x^{*}\right) \varphi_{F}^{(1)}\left(a_{1} ; l_{1}\right) \cdots \varphi_{F}^{(n)}\left(a_{n} ; l_{n}\right) \psi_{\varepsilon}^{(\nu)}(x)\right\rangle_{A}$, 


$$
\begin{aligned}
& w_{F \pm}(x ; L, \Lambda)_{\mu \nu}=\left\langle\varphi_{F}^{(1)}\left(a_{1} ; l_{1}\right) \cdots \varphi_{l_{\mu}}^{F(\mu)}\left(a_{\mu} ; l_{\mu}\right) \cdots \varphi_{F}^{(n)}\left(a_{n} ; l_{n}\right) \psi_{ \pm}^{(\nu)}(x)\right\rangle_{\Lambda}, \\
& w_{F \pm}^{*}\left(x^{*} ; L, \Lambda\right)_{\mu \nu}=\left\langle\psi_{ \pm}^{*(\mu)}\left(x^{*}\right) \varphi_{F}^{(1)}\left(a_{1} ; l_{1}\right) \cdots \varphi_{-l_{\nu}}^{F(\nu)}\left(a_{\nu} ; l_{\nu}\right) \cdots \varphi_{F}^{(n)}\left(a_{n} ; l_{n}\right)\right\rangle_{\Lambda} .
\end{aligned}
$$

The $\tau$ function reads

$$
\tau_{F}(L, \Lambda)=\left\langle\varphi_{F}^{(1)}\left(a_{1} ; l_{1}\right) \cdots \varphi_{F}^{(n)}\left(a_{n} ; l_{n}\right)\right\rangle_{\Lambda} .
$$

Assuming $l_{1}, \cdots, l_{n} \notin \mathbb{Z}+1 / 2$ we give local expansions for (4. 4. 38)

(4. 4. 40)。 $w_{F}\left(x^{*}, x ; L, \Lambda\right) \equiv \frac{-i \tau_{F}(L ; \Lambda)}{\pi}\left(-\widetilde{w}_{\frac{1}{2}}^{*}\left[x^{*}\right], \widetilde{w}_{\frac{1}{2}}\left[x^{*}\right]\right) \otimes \Lambda$,

$(4.4 .40)_{\nu} \quad w_{F}^{( \pm)}\left(x^{*}, x ; L, \Lambda\right)^{(*)} \equiv \sum_{j=1}^{\infty} \widetilde{C}_{j, \Lambda}\left(w_{F}^{(t)}\right) E_{\nu} \Lambda \cdot w_{-l_{\nu}+j}\left[a_{\nu}\right]$

$$
\begin{gathered}
+\sum_{j=0}^{\infty} \widetilde{C}_{j, \Lambda}^{*}\left(w_{F^{\prime}}^{( \pm)}\right) E_{\nu} \Lambda \cdot w_{l_{\nu}+j}^{*}\left[a_{\nu}\right], \\
\left\{\begin{array}{lc}
\widetilde{C}_{j, \Lambda}\left(w_{F}^{( \pm)}\right)_{\mu \nu}=\left\langle\psi_{ \pm}^{*(\mu)}\left(x^{*}\right) \varphi_{F}^{(1)}\left(a_{1} ; l_{1}\right) \cdots \varphi_{-l_{\nu}+j}^{(\nu)}\left(a_{\nu} ; l_{\nu}\right) \cdots \varphi_{F}^{(n)}\left(a_{n} ; l_{n}\right)\right\rangle_{\Lambda} \\
\widetilde{C}_{j, \Lambda}^{*}\left(w_{F}^{( \pm)}\right)=\widetilde{C}_{-j, \Lambda}\left(w_{F}^{( \pm)}\right) & (j \geqq 0),
\end{array}\right.
\end{gathered}
$$

(4. 4. 41),

$$
\begin{aligned}
& w_{F}(x ; L, \Lambda) \equiv \frac{-i \tau_{F}(L, \Lambda)}{2 \cos \pi l_{\nu}} E_{\nu} \Lambda \cdot w_{-l_{\nu}}\left[a_{\nu}\right] \\
& +\sum_{j=1}^{\infty} C_{j, \Lambda}\left(w_{F}\right) E_{\nu} \Lambda \cdot w_{-l_{\nu}+j}\left[a_{\nu}\right]+\sum_{j=0}^{\infty} C_{j, \Lambda}^{*}\left(w_{F}\right) E_{\nu} \Lambda \cdot w_{l_{\nu}+j}^{*}\left[a_{\nu}\right], \\
& \left(\begin{array}{rlr}
C_{j, \Lambda}\left(w_{F}\right)_{\mu \nu}= \pm\left\langle\varphi_{F}^{(1)}\left(a_{1} ; l_{1}\right)\right. & \cdots \varphi_{l_{\mu}}^{F *(\mu)}\left(a_{\mu} ; l_{\mu}\right) \cdots \varphi_{-l_{\nu+j}}^{F(\nu)}\left(a_{\nu} ; l_{\nu}\right) \\
& \left.\cdots \varphi_{F}^{(n)}\left(a_{n} ; l_{n}\right)\right\rangle_{\Lambda} \quad(\mu \lessgtr \nu, j \in \mathbb{Z})
\end{array}\right.
\end{aligned}
$$

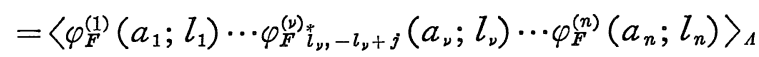

$$
\begin{aligned}
& (\mu=\nu, j \in \mathbb{Z}) \\
& C_{j, \Lambda}^{*}\left(w_{F}\right)=\frac{i \tau_{F}(L ; \Lambda)}{2 \cos \pi L} \delta_{j 0}+C_{-j, \Lambda}\left(w_{F}\right) \quad(j \geqq 0),
\end{aligned}
$$

$(4.4 .42)_{\nu}{ }^{t} w_{F}^{*}\left(x^{*} ; L, \Lambda\right) \equiv \sum_{j=0}^{\infty} C_{j, \Lambda}\left({ }^{t} w_{F}^{*}\right) E_{\nu} \Lambda \cdot w_{l_{\nu}+j}\left[a_{\nu}\right]$

$$
\begin{aligned}
& -\frac{i \tau_{F}(L ; \Lambda)}{2 \cos \pi l_{\nu}} E_{\nu} \Lambda \cdot w_{-l_{\nu}}^{*}\left[a_{\nu}\right] \\
& +\sum_{j=1}^{\infty} C_{j, \Lambda}^{*}\left({ }^{t} w_{F}^{*}\right) E_{\nu} \Lambda \cdot w_{-l_{\nu}+j}^{*}\left[a_{\nu}\right],
\end{aligned}
$$

(*) ${ }^{t} w_{F}^{( \pm)}\left(x^{*}, x ; L, \Lambda\right)=\left({ }^{t} w_{F \pm,+}\left(x^{*}, x ; L, \Lambda\right),{ }^{t} w_{F_{ \pm},-}\left(x^{*}, x ; L, \Lambda\right)\right)$. 


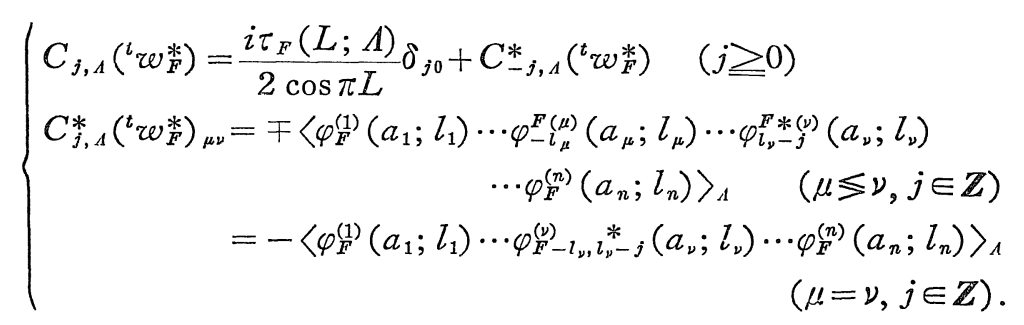

Therefore if we set

$$
\begin{aligned}
& \widehat{w}_{F}^{( \pm)}\left(x^{*}, x ; L, \Lambda\right)=\pi i \mho_{F}^{( \pm)}\left(x^{*}, x ; L, \Lambda\right) / \tau_{F}(L, \Lambda), \\
& \widehat{w}_{F}(x ; L, \Lambda)=2 i \cos \pi L \cdot w_{F}(x: L, \Lambda) / \tau_{F}(L, \Lambda), \\
& { }^{t} \widehat{w}_{F}^{*}\left(x^{*} ; L, \Lambda\right)=2 i \cos \pi L \cdot{ }^{t} w_{F}^{*}\left(x^{*} ; L, \Lambda\right) / \tau_{F}(L, \Lambda) .
\end{aligned}
$$

Their $\mu$-th row (resp. column) vectors $\widehat{w}_{F^{\mu}}^{( \pm)}\left(x^{*}, x ; L, \Lambda\right), \widehat{w}_{F^{\prime}}(x ; L, \Lambda)$ (resp. $\widehat{\imath}_{F}^{* \mu}\left(x^{*} ; L, \Lambda\right)$ ) provide the canonical independent elements of $W_{F^{*}, z^{*}, a_{1}, \cdots, a_{n}}^{( \pm) 0, l_{n}, \ldots, l_{n} \text { strict }}\left(\Lambda, \lambda_{(\mu)}\right)$ (resp. $\left.W_{F, a_{1}, \cdots, a_{n}}^{*,, l_{1}, \cdots, l_{n}, \text { strict }}(\Lambda)\right)$ in the sense that $\tilde{c}_{0}^{(0)}\left(\widehat{w}_{F^{\mu}}^{( \pm)}\right)$ $=0, \widetilde{c}_{-l_{\nu}}^{(\nu)}\left(\mathcal{v V}_{F^{\mu}}^{( \pm)}\right)=0, \widetilde{c}_{-l_{\nu}}^{(\nu)}\left(\widehat{\boldsymbol{v}}_{F_{\mu} \mu}\right)=\delta_{\mu \nu}, \tilde{c}_{-l_{\nu}}^{*(\nu)}\left({ }^{t} \widehat{w}_{F_{\mu}}^{*}\right)=\delta_{\mu \nu} . \quad$ In correspondence with (4.4.16)-(4.4.18) we have (cf. (3.2.44))

(4. 4. 44) $\quad \alpha_{0 \nu}^{( \pm)}\left(L+\frac{1}{2}, \Lambda, \lambda_{\mu}\right)=\pi i\left\langle\psi_{ \pm}^{*(\mu)}\left(x^{*}\right) \varphi_{F}^{(1)}\left(a_{1} ; l_{1}\right) \cdots \varphi_{-l_{\nu}+1}^{F^{(\nu)}}\left(a_{\nu} ; l_{\nu}\right)\right.$

$$
\begin{array}{r}
\cdots \varphi_{F}^{(n)}\left(a_{n} ; l_{n}\right)>/ \tau_{F}(L, \Lambda), \\
\beta_{0 \nu}^{( \pm)}\left(L+\frac{1}{2}, \Lambda, \lambda_{\mu}\right)=\frac{\pi}{2 \cos \pi l_{\nu}} \widehat{\omega}_{F, \pm}^{*}\left(x^{*} ; L, \Lambda\right)_{\mu \nu}
\end{array}
$$

(4. 4. 45) $\quad \alpha_{\mu \nu}\left(L+\frac{1}{2}, \Lambda\right)=2 i \cos \pi l_{\mu} \cdot \tau_{F_{\mu \nu}^{*}}\left(L, \Lambda ; l_{\mu},-l_{\nu}+1\right) / \tau_{F}(L, \Lambda)$,

$$
\beta_{\mu \nu}\left(L+\frac{1}{2}, \Lambda\right)=-\delta_{\mu \nu}+2 i \cos \pi l_{\mu} \cdot \tau_{F_{\mu \nu}^{*}}\left(L, \Lambda ; l_{\mu},-l_{\nu}\right) / \tau_{F}(L, \Lambda)
$$

where

(4. 4. 46) $\quad \tau_{F_{\mu \nu}^{*}}\left(L, \Lambda ; l, l^{\prime}\right)= \pm\left\langle\varphi_{F}^{(1)}\left(a_{1} ; l_{1}\right) \cdots \varphi_{l}^{F *(\mu)}\left(a_{\mu} ; l_{\mu}\right)\right.$

$$
\begin{gathered}
\left.\cdots \varphi_{l^{\prime}}^{F(\nu)}\left(a_{\nu} ; l_{\nu}\right) \cdots \varphi_{F}^{(n)}\left(a_{n} ; l_{n}\right)\right\rangle_{A} \quad(\mu \lessgtr \nu) \\
=\left\langle\varphi_{F}^{(1)}\left(a_{1} ; l_{1}\right) \cdots \varphi_{F_{l l^{*}}^{*}}^{(\mu)}\left(a_{\mu} ; l_{\mu}\right)\right. \\
\left.\cdots \varphi_{F}^{(n)}\left(a_{n} ; l_{n}\right)\right\rangle_{A} \quad(\mu=\nu) .
\end{gathered}
$$

The relations (4.4.19)-(4.4.23) are all valid with obvious modifications.

Our original cases $(4.4 .1)-(4.4 .3)$ are obtained as the special case 
$\lambda_{\mu \nu}=1$ for all $\mu, \nu=1, \cdots, n$ (cf. Proposition 3.2.14).

Consider now the most general wave functions

$$
\left\langle\phi^{*\left(\kappa_{1}\right)} \cdots \phi^{*\left(\kappa_{k}\right)} \varphi_{B}^{(1)} \cdots \varphi^{B\left(\nu_{1}\right)} \cdots \varphi^{B\left(\nu_{m}\right)} \cdots \varphi_{B}^{(n)} \phi^{\left(\mu_{1}\right)} \cdots \phi^{\left(\mu_{l}\right)}\right\rangle_{A} / \tau_{B}(L, \Lambda),
$$

$$
\left\langle\psi_{\varepsilon_{1}^{*}}^{*\left(\kappa_{1}\right)} \cdots \psi_{\varepsilon_{k}^{*}}^{*\left(\kappa_{k}\right)} \varphi_{F}^{(1)} \cdots \varphi^{F\left(\nu_{1}\right)} \cdots \varphi^{F\left(\nu_{m}\right)} \cdots \varphi_{F}^{(n)} \phi_{\varepsilon_{1}}^{\left(\mu_{1}\right)} \cdots \phi_{\varepsilon_{l}}^{\left(\mu_{l}\right)}\right\rangle_{A} / \tau_{F}(L, \Lambda) .
$$

In (4. 4. 47) we have set $\phi^{*\left(\kappa_{i}\right)}=\phi^{*\left(\kappa_{i}\right)}\left(x_{i}^{*}\right), \varphi_{B}^{(\nu)}=\varphi_{B}^{(\nu)}\left(a_{\nu} ; l_{\nu}\right), \phi^{\left(\mu_{j}\right)}=\phi^{\left(\mu_{j}\right)}\left(x_{j}\right)$, and the fields $\varphi^{B\left(\nu_{k}\right)}$, which stands either for $\varphi_{-\nu_{\nu_{k}}}^{B\left(\nu_{k}\right)}\left(a_{\nu_{k}} ; l_{\nu_{k}}\right)$ or for $\varphi_{\nu_{\nu_{k}}}^{B^{*}\left(\nu_{k}\right)}\left(a_{\nu_{k}} ; l_{\nu_{k}}\right)$, are placed at $\nu=\nu_{1}, \cdots, \nu_{m}$. Similar convention is used in (4. 4. 48), where $\varepsilon_{i}^{*}, \varepsilon_{j}=+$ or -. Notice that (4.4.47), (4.4.48) reduce to 0 unless $k+m+l$ and the number of the starred operators $\phi^{*\left(\kappa_{i}\right)}$, $\varphi^{B *\left(\nu_{k}\right)}\left(\right.$ or $\left.\psi^{*\left(\kappa_{i}\right)}, \varphi^{F *\left(\nu_{k}\right)}\right)$ are both even. By the same reasoning as above we see that the Euclidean continuation of (4.4.47), viewed as a function of $\left(z_{1}, \bar{z}_{1}\right)=\left(-x_{1}^{-}, x_{1}^{+}\right)$, gives a $\mu_{1}$-th element of a function belonging to $W_{B, z_{1}, \cdots, \cdots, z_{k}^{*}, a_{1}, \cdots, a_{n}}^{0, \ldots, 0, l_{1}, \ldots, l_{n}, \text { strict }}\left(\Lambda, \lambda_{\left(\kappa_{1}\right)}, \cdots, \lambda_{\left(\kappa_{i}\right)}\right)=\bigoplus_{i=0}^{k} W_{B, z_{i}, \cdots, a_{1}, \cdots, a_{n}}^{0, l_{1}, \ldots, l_{n}, \text { strict }}\left(\Lambda, \lambda_{\left(\kappa_{i}\right)}\right) \quad\left(\lambda_{\left(\kappa_{i}\right)}=\kappa_{i}\right.$ th row of $\Lambda)$, and hence is a linear combination of $\widehat{w}_{B}\left(z_{i}^{*}, z_{1}, L, \Lambda, \lambda_{\left(\kappa_{i}\right)}\right) \kappa_{i \mu}$ $(1 \leqq i \leqq k), \widehat{w}_{B}\left(z_{1} ; L, \Lambda\right)_{\mu \mu_{1}}(1 \leqq \mu \leqq n)$. The same argument applies separately to each variable $x_{i}^{*}$ or $x_{j}$. Indeed we have the following formula, which is a direct consequence of (A. 27):

(4. 4. 49) $\quad \widehat{w}_{B, n_{1}, \cdots, \kappa_{k}, \mu_{1}, \cdots, \mu_{l}}^{\nu_{1}, \ldots, \nu_{m}}$

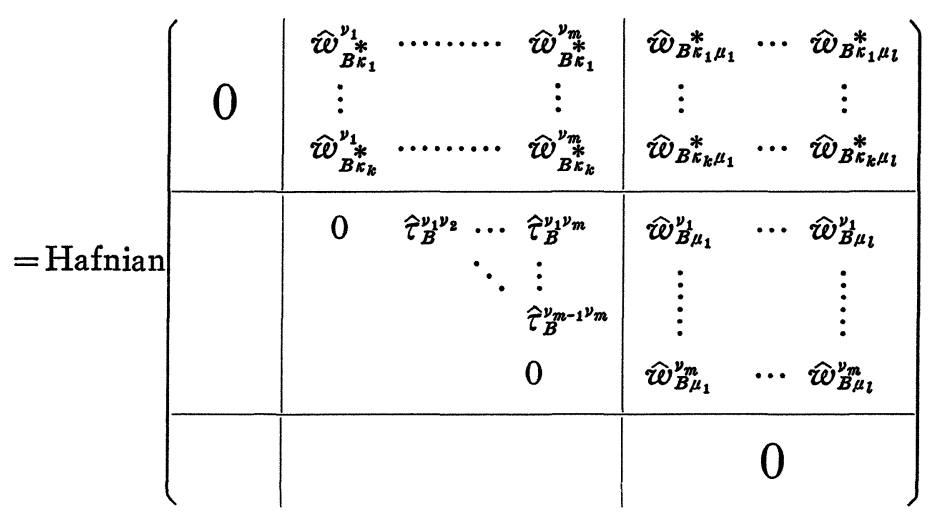

where $\widehat{\boldsymbol{w}}_{B, k_{1}, \ldots, k_{k}, \mu_{1}, \cdots, \mu_{l}}^{\nu_{1}, \ldots, \nu_{m}}$ denotes (4. 4. 47), and

$$
\begin{aligned}
& \widehat{\omega}_{B \hbar}^{\nu *}=w_{B}^{*}\left(x_{\kappa}^{*} ; L, \Lambda\right)_{\kappa \nu} / \tau_{B}(L, \Lambda), \\
& \widehat{w}_{B \kappa \mu}^{*}=w_{B}\left(x_{\kappa}^{*}, x_{\mu} ; L, \Lambda\right)_{\kappa \mu} / \tau_{B}(L, \Lambda), \\
& \widehat{\omega}_{B \mu}^{\nu}=w_{B}\left(x_{\mu} ; L, \Lambda\right)_{\nu \mu} / \tau_{B}(L, \Lambda),
\end{aligned}
$$




$$
\hat{\tau}_{B}^{\mu \nu}=\left\langle\varphi_{B}^{(1)} \cdots \varphi^{B(\mu)} \cdots \varphi^{B(\nu)} \cdots \varphi_{B}^{(n)}\right\rangle .
$$

Note that $\hat{\tau}_{B}^{\mu \nu}=\tau_{B \mu \nu}^{*}\left(L, \Lambda ; l_{\mu},-l_{\nu}\right) / \tau_{B}(L, \Lambda)$ if $\varphi^{\Delta(a)}$ is starred and $\varphi^{B(\nu)}$ is not. For a symmetric matrix $\left(a_{i j}\right)_{1 \leq i, j \leqslant k}$ we set Hafnian $\left(a_{i j}\right)=0 \quad(k$ : odd), $=\sum_{\text {pairings }} a_{j_{1} j_{2}} \cdots a_{j_{k-1} j_{k}}$ ( $k$ : even), and the lower triangle of (4.4.49) is omitted under the convention that the matrix inside is symmetric.

Likewise in the Fermi case we have

(4. 4.51) $\widehat{w}_{F, k_{1}, \cdots, \kappa_{k} \mu_{1}, \cdots, \mu_{l}}^{\nu_{1}, \ldots, \nu_{m}}$

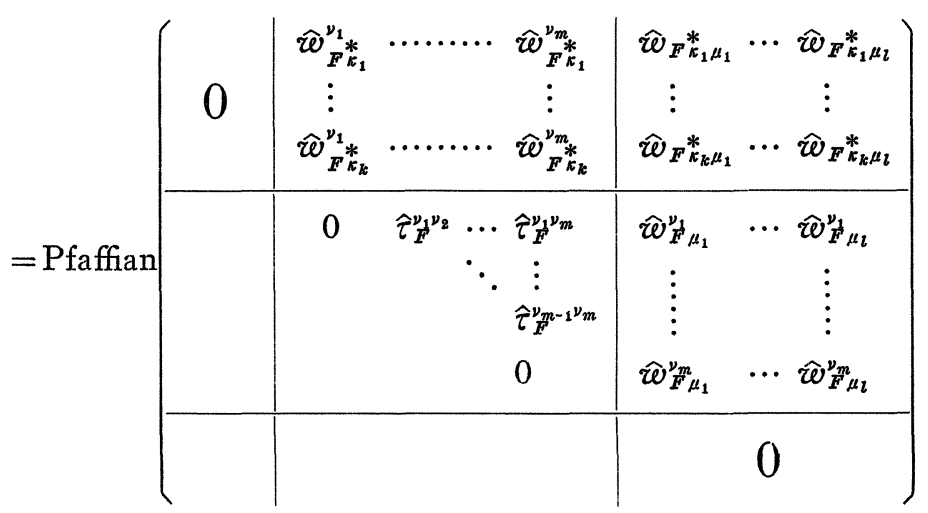

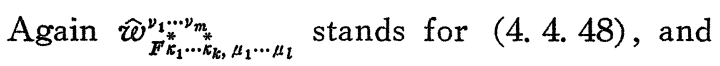

$$
\begin{aligned}
& \widehat{\mho}_{F *}^{\nu *}=w_{F}^{*}\left(x_{\kappa}^{*} ; L, \Lambda\right)_{\kappa \nu} / \tau_{F}(L, \Lambda), \\
& \widehat{w}_{F^{*} \mu}^{*}=w_{F}\left(x_{\kappa}^{*}, x_{\mu} ; L, \Lambda\right)_{\kappa \mu} / \tau_{F}(L, \Lambda), \\
& \widehat{\mho}_{F \mu}^{\nu}=w_{F}\left(x_{\mu} ; L, \Lambda\right)_{\nu \mu} / \tau_{F}(L, \Lambda), \\
& \hat{\tau}_{F}^{\mu \nu}=\left\langle\varphi_{F}^{(1)} \cdots \varphi^{F(\mu)} \cdots \varphi^{F(\nu)} \cdots \varphi_{F}^{(n)}\right\rangle .
\end{aligned}
$$

In (4. 4.51) and (4.4.52) we have omitted to indicate $\varepsilon_{i}^{*}$, $\varepsilon_{j}$ for simplicity. For a skew symmetric matrix $\left(a_{i j}\right)_{1 \leqq i, j \leqq k}$ we set Pfaffian $\left(a_{i j}\right)=0$ ( $k$ : odd), = $\sum_{\text {pairings }} \operatorname{sgn}\left(\begin{array}{c}12 \ldots k \\ j_{1} j_{2} \cdots j_{k}\end{array}\right) a_{j_{1} j_{2}} \cdots a_{j_{k-1} j_{k}}(k: \text { even })^{(*)}$, and the lower triangle of (4.4.51) is omitted under the convention that the matrix inside is skew-symmetric.

Formulas (4. 4. 49) - (4.4.52) tell that the quantities $w_{B}\left(x^{*}, x ; L, \Lambda\right)$, $w_{B}(x ; L, \Lambda), w_{B}^{*}\left(x^{*} ; L, \Lambda\right), \tau_{B \mu \nu}^{*}\left(L, \Lambda ; l_{\mu},-l_{\nu}\right)$ and their Fermi versions are elementary. On the other hand, these are characterized by linear and

(*) As is well known (Pfaffian $A)^{2}=\operatorname{det} A$, but Hafnian $A$ is not related to det $A$. 
non-linear total differential equations according to the results of Chapter III. Thus we have a complete characterization of arbitrary wave- and $\tau$ functions.

\section{$\S 4.5 . \quad$ Convergence of Products}

In this section we exploit the product formula to define rigorously the product of our field operators. In particular, we obtain infinite series expressions for $\tau$ functions and wave functions. Our idea of convergence proof is the same as that of Section 2.3. Contrary to the divergence of (2.2.28) in 1-dimensional case, $\tau$ function itself has a convergent series expression here. We shall mainly deal with the symplectic case. The orthogonal case is similarly treated.

Following the notations in the previous section, we set

$$
R_{B}\left(u, u^{\prime}\right)=\left(\begin{array}{lll}
R_{B}^{(1)}\left(u, u^{\prime}\right) & & \\
& \ddots & \\
& & R_{B}^{(n)}\left(u, u^{\prime}\right)
\end{array}\right)
$$

where

$$
=e^{-i m\left(a_{\nu}^{-} u+a_{\nu}^{+} u^{-1}\right)}(u-i 0)^{-l_{\nu}+1} \cdot \frac{-2 \sin \pi l_{\nu}}{u+u^{\prime}-i 0} \cdot e^{-i m\left(a_{\nu}^{-} u^{\prime}+a_{\nu}^{+} u^{\prime-1}\right.}\left(u^{\prime}-i 0\right)^{l_{\nu}},
$$

(4. 5.2) $)_{B} \quad A_{B}\left(u, u^{\prime}\right)$

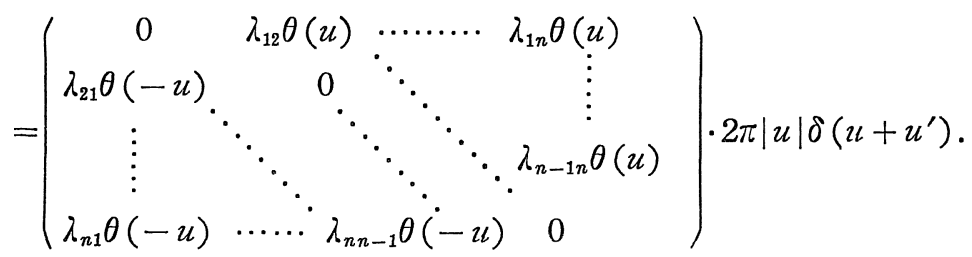

From (A. 24) we have the following infinite series for $\log \tau_{B}=$ $\log \tau_{B}\left(a_{1}, \cdots, a_{n} ; L, \Lambda\right)$.

(4.5.3) $\log \tau_{B}=2 \sum_{l=2}^{\infty} \frac{1}{2 l} \int \cdots \int d u_{1} \cdots d u_{2 l} \operatorname{trace} A_{B}\left(u_{1}, u_{2}\right) R_{B}\left(u_{2}, u_{3}\right)$

$$
\cdots A_{B}\left(u_{2 l-1}, u_{2 l}\right) R_{B}\left(u_{2 l}, u_{1}\right) \text {. }
$$

We set $\varepsilon_{\mu \nu}= \pm 1$ if $\mu \gtrless \nu,=0$ if $\mu=\nu$, and define 
(4. 5. 4) $\quad e_{\mu \nu}(u)=\lambda_{\mu \nu} \varepsilon_{\mu \nu} \theta\left(-\varepsilon_{\mu \nu} u\right)$

$$
\begin{aligned}
& \times e^{-\pi i\left(\left(\varepsilon_{\mu \nu}+1\right) l_{\mu} / 2+\left(\varepsilon_{\mu \nu}-1\right) l_{\nu} / 2\right)}|u|^{l_{\mu}-l_{\nu}} \\
& \times e^{-i m\left(\left(a_{\mu}-a_{\nu}\right)-u+\left(a_{\mu}-a_{\nu}\right)+u-1\right)},
\end{aligned}
$$

(4. 5. 5) ${ }_{B} \quad E_{B}(u)=\left(e_{\mu \nu}(u)\right)_{\mu, \nu=1, \ldots, n}$,

$(4.5 .6)_{B}$

$$
P_{B}\left(u, u^{\prime}\right)=\left(\begin{array}{lll}
2 \sin \pi l_{1} & & \\
& \ddots & \\
& & 2 \sin \pi l_{n}
\end{array}\right) \frac{1}{u-u^{\prime}+i 0} .
$$

Then (4.5.3) is rewritten as

$$
\text { (4. 5. 7) } \begin{aligned}
\log \tau_{B}= & 2 \sum_{l=2}^{\infty} \frac{1}{2 l} \int \cdots \int \frac{d u_{1}}{2 \pi} \cdots \frac{d u_{l}}{2 \pi} \\
& \times \text { trace } E_{B}\left(u_{1}\right) P_{B}\left(u_{1}, u_{2}\right) \cdots E_{B}\left(u_{l}\right) P_{B}\left(u_{l}, u_{1}\right) .
\end{aligned}
$$

Let $U_{0}$ be a relatively compact open subset in $Y^{n, \boldsymbol{C}} \times \mathbb{C}^{n} \times \mathbb{C}^{n(n-1) / 2}$ where we identify $\mathbb{C}^{n}$ and $\mathbb{C}^{n(n-1) / 2}$ with the parameter spaces $\{L$ $\left.=\left(\delta_{\mu \nu} l_{\nu}\right)_{\mu, \nu=1, \cdots, n}\right\}$ and $\left\{\Lambda=\left(\lambda_{\mu \nu}\right)_{\mu, \nu=1, \cdots, n} \mid \lambda_{\mu \nu}=\lambda_{\nu \mu}, \lambda_{\nu \nu}=1 \quad(\mu, \nu=1, \cdots, n)\right\}$ respectively.

We denote by $E_{\mu \nu}$ and $P_{\nu}$ the bounded linear operators in $L^{2}(\boldsymbol{R} \times \mathbb{R})$ $=L^{2}\left(\boldsymbol{R} \times \boldsymbol{R} ;(d u / 2 \pi) \times\left(d u^{\prime} / 2 \pi\right)\right)$ given by

$$
\left(E_{\mu \nu} f\right)\left(u, u^{\prime}\right)=e_{\mu \nu}(u) f\left(u, u^{\prime}\right)
$$

and

$$
\left(P_{\nu} f\right)\left(u, u^{\prime}\right)=\int_{-\infty}^{\infty} \frac{d u^{\prime \prime}}{2 \pi} \frac{2 \sin \pi l_{\nu}}{u-u^{\prime \prime}+i 0} f\left(u^{\prime \prime}, u^{\prime}\right)
$$

respectively. We set also

$$
\begin{aligned}
& C_{1}=\max _{\mu, \nu=1, \cdots, n} \sup _{U_{0}}\left\|E_{\mu \nu}\right\|_{L^{2}(\boldsymbol{R} \times \boldsymbol{R})}, \\
& C_{2}=\max _{\nu=1, \cdots, n} \sup _{U_{0}}\left\|P_{\nu}\right\|_{L^{2}(\boldsymbol{R} \times \boldsymbol{R})},
\end{aligned}
$$

where $\|\cdot\|_{L^{2}(\boldsymbol{R} \times \boldsymbol{R})}$ denotes the operator norm.

Proposition 4. 5. 1. We assume that $C_{1} C_{2}<\frac{1}{n}$. Then the right hand side of (4.5.7) is uniformly convergent in $U_{0}$.

Proof. The integrand of $l$-th term in (4.5.7) consists of $n^{l}$ functions of the form 
(4. 5. 8) $\quad F_{\mu_{1} \cdots \mu_{l}}\left(u_{1}, \cdots, u_{l}\right)=e_{\mu_{1} \mu_{2}}\left(u_{1}\right) \frac{2 \sin \pi l_{\mu_{2}}}{u_{1}-u_{2}+i 0}$

$$
\times e_{\mu_{2} \mu_{3}}\left(u_{2}\right) \frac{2 \sin \pi l_{\mu_{3}}}{u_{2}-u_{3}+i 0} \cdots c_{\mu_{l} \mu_{1}}\left(u_{l}\right) \frac{2 \sin \pi l_{u_{1}}}{u_{l}-u_{1}+i 0} .
$$

It is sufficient for us to prove

$$
\text { (4.5.9) }\left|\int \cdots \int \frac{d u_{1}}{2 \pi} \cdots \frac{d u_{l}}{2 \pi} F_{\mu_{1} \cdots \mu_{l}}\left(u_{1}, \cdots, u_{l}\right)\right| \leqq C\left(\frac{\varepsilon}{n}\right)^{l-2}
$$

for some $\varepsilon$ such that $0<\varepsilon<1$, and $C>0$. Without loss of generality we assume that $\varepsilon_{\mu_{l} \mu_{1}} \cdot \varepsilon_{\mu_{1} \mu_{2}}=-1$. Let $m$ be the integer such that $\varepsilon_{\mu_{1} \mu_{2}} \cdot \varepsilon_{\mu_{2} \mu_{3}}$ $=\cdots=\varepsilon_{\mu_{m-1} \mu_{m}} \varepsilon_{\mu_{m} \mu_{m+1}}=1$ and $\varepsilon_{\mu_{m} \mu_{m+1}} \cdot \varepsilon_{\mu_{m+1} \mu_{m+2}}=-1$. Then we have $m<l$. We assume that $\varepsilon_{\mu_{1} \mu_{2}}=-1$. (The other cases are similarly treated.)

We set

(4. 5. 10) $f_{1}\left(u_{1}, u_{m+1}\right)=\int_{0}^{\infty} \frac{d u_{2}}{2 \pi} \cdots \int_{0}^{\infty} \frac{d u_{m}}{2 \pi}$

$$
\begin{aligned}
& \times \frac{2 \sin \pi l_{\mu_{2}}}{u_{1}-u_{2}+i 0} e_{\mu_{2} \mu_{s}}\left(u_{2}\right) \cdots-\frac{2 \sin \pi l_{\mu_{m}}}{u_{m-1}-u_{m}+i 0} \\
& \times e_{\mu_{m} \mu_{m+1}}\left(u_{m}\right) \frac{2 \sin \pi l_{\mu_{m+1}} \theta\left(-u_{m+1}\right),}{u_{m}-u_{m+1}}
\end{aligned}
$$

(4. 5. 11) $\quad g_{1}\left(u_{m+1}, u_{1}\right)=e_{\mu_{m+1} \mu_{m+2}}\left(u_{m+1}\right) \int_{-\infty}^{\infty} \frac{d u_{m+2}}{2 \pi} \cdots \int_{-\infty}^{\infty} \frac{d u_{l}}{2 \pi}$

$$
\times \frac{2 \sin \pi l_{\mu_{m+2}}}{u_{m+1}-u_{m+2}+i 0} \cdots e_{\mu_{l} \mu_{1}}\left(u_{l}\right) \frac{2 \sin \pi l_{\mu_{1}}}{u_{l}-u_{1}} e_{\mu_{1} \mu_{2}}\left(u_{1}\right) \text {. }
$$

For $\left(a_{1}, \cdots, a_{n}\right) \in Y^{n, \boldsymbol{C}}, \boldsymbol{e}_{\mu \nu}(u)$ is exponentially decreasing as $|u| \rightarrow 0$ or $\infty$. Hence

$$
f_{0}\left(u, u^{\prime}\right)=e_{\mu_{m} \mu_{m^{+1}}}(u) \frac{2 \sin \pi l_{\mu_{m+1}}}{u-u\left(\iota^{\prime}\right.}\left(-u^{\prime}\right)
$$

and

$$
g_{0}\left(u, u^{\prime}\right)=e_{\mu_{l} \mu_{1}}(u) \frac{2 \sin \pi l_{\mu_{1}}}{u-u^{\prime}} e_{\mu_{1} \mu_{2}}\left(u^{\prime}\right)
$$

belong to $L^{2}(\mathbb{R} \times \mathbb{R})$. We set

$$
C_{3}=\max _{\mu, \nu=1, \cdots, n} \sup _{\sigma_{0}}\left\|e_{\mu \nu}(u) \frac{2 \sin \pi l_{\nu}}{u-u^{\prime}} \theta\left(\varepsilon_{\mu \nu} u^{\prime}\right)\right\|_{L^{2}(\boldsymbol{R} \times \boldsymbol{R})},
$$




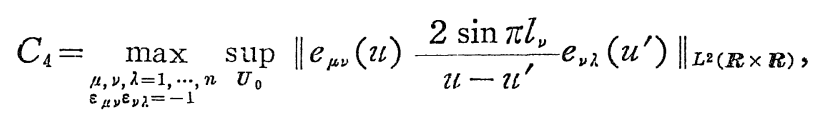

where $\|\cdot\|_{L^{2}(\boldsymbol{R} ; \boldsymbol{R})}$ denotes the norm in $L^{2}(\boldsymbol{R} \times \mathbb{R})$. Since $f_{1}=P_{\mu_{2}} E_{\mu_{2} / \mu_{3}} \cdots$ $P_{\mu_{m}} f_{0}$ and $g_{1}=E_{\mu_{m+1} \mu_{m+2}} P_{\mu_{m+2}} \cdots P_{\mu_{l}} g_{0}$ belong to $L^{2}(\mathbb{R} \times \mathbb{R})$, we have the following estimate.

$$
\begin{aligned}
& \int \cdots \int \frac{d u_{1}}{2 \pi} \cdots \frac{d u_{l}}{2 \pi} F_{\mu_{1} \cdots \mu_{l}}\left(u_{1}, \cdots, u_{l}\right) \mid \\
& =\left|\iint \frac{d u_{1}}{2 \pi} \frac{d u_{m+1}}{2 \pi} f_{1}\left(u_{1}, u_{m+1}\right) g_{1}\left(u_{m+1}, u_{1}\right)\right| \\
& \leqq\left\|f_{1}\right\|_{L^{2}(\boldsymbol{R} \times \boldsymbol{R})} \cdot\left\|g_{1}\right\|_{L^{2}(\boldsymbol{R} \times \boldsymbol{R})} \leqq C_{1}^{l-9} C_{2}^{l-2} C_{3} C_{4} .
\end{aligned}
$$

Thus we have shown (4.5.9).

Choose $\nu_{1}, \cdots, \nu_{k}$ so that $1 \leqq \nu_{1}<\nu_{2}<\cdots<\nu_{k}<n$. Let us consider $\log \tau_{B}$ in the limit

$$
\min _{1 \leqq j \leqq k} \operatorname{Im}\left(a_{\nu_{j}+1}-a_{\nu_{j}}\right)^{ \pm} \rightarrow \infty
$$

We denote by $\Lambda_{j+1}(j=0, \cdots, k-1)$ the submatrix $\left(\begin{array}{c}\lambda_{\nu_{j}+1 \nu_{j}+1} \cdots \lambda_{\nu_{j}+1 \nu_{j+1}} \\ \vdots \\ \lambda_{\nu_{j}+1 \nu_{j+1}} \cdots \lambda_{\nu_{j+1} \nu_{j+1}}\end{array}\right)$ $\left(\nu_{0}=0\right)$, and by $L_{j+1}(j=0, \cdots, k-1)$ the submatrix $\left(\delta_{\mu \nu} l_{\nu}\right)_{\nu_{j}+1 \leq \mu, \nu \leq \nu_{j+1}}$. Since in the limit (4.5.12) $e_{\mu \nu}(u)$ tends to 0 except when $\nu_{j}<\mu, \nu$ $\leqq \nu_{j+1}$ for some $j$, the following cluster property follows from Proposition 4. 5. 1 and its orthogonal version.

Proposition 4. 5. 2. In the limit (4.5.12) we have for $*=B$ or F

$$
\begin{aligned}
& \lim \log \tau_{*}\left(a_{1}, \cdots, a_{n} ; L, \Lambda\right) \\
& \quad=\sum_{j=1}^{k} \log \tau_{*}\left(a_{\nu_{j+1}}, \cdots, a_{\nu_{j+1}} ; L_{j}, \Lambda_{j}\right) .
\end{aligned}
$$

Denoting by $d$ the exterior differentiation with respect to $\left(a_{1}, \cdots, a_{n}\right)$, we have

(4.5.14) $\quad d \log \tau_{B}=\sum_{l=2}^{\infty} \int \cdots \int \underline{d u_{1}} \cdots \underline{d u_{l}}$ 


$$
\begin{aligned}
& \times \operatorname{trace} A_{B}\left(u_{1}, u_{2}\right) R_{B}\left(u_{2}, u_{3}\right) \cdots A_{B}\left(u_{2 l-1}, u_{2 l}\right) d R_{B}\left(u_{2 l}, u_{1}\right) \\
& =\sum_{l=2}^{\infty} \int_{-\infty}^{\infty} \cdots \int_{-\infty}^{\infty} \frac{d u_{1}}{2 \pi} \cdots \frac{d u_{l}}{2 \pi} \\
& \times\left\{\operatorname{trace} E_{B}\left(u_{1}\right) P_{B}\left(u_{1}, u_{2}\right) \cdots E_{B}\left(u_{l}\right)\left(\begin{array}{c}
-2 i \sin \pi l_{1} d\left(-m a_{1}^{-}\right) \\
-2 i \sin \pi l_{n} \dot{d}\left(-m a_{n}^{-}\right)
\end{array}\right)\right. \\
& +\operatorname{trace} u_{1}^{-1} E_{B}\left(u_{1}\right) P_{B}\left(u_{1}, u_{2}\right) \cdots E_{B}\left(u_{l}\right) u_{l}^{-1} \\
& \left.\times\left(\begin{array}{c}
-2 i \sin \pi l_{1} d\left(m a_{1}^{+}\right) \\
\ddots \\
-2 i \sin \pi \dot{l}_{n} d\left(m a_{n}^{+}\right)
\end{array}\right)\right\} .
\end{aligned}
$$

Once the convergence of $\log \tau_{B}$ is shown in a region, $\log \tau_{B}$ is analytically prolongable as long as $d \log \tau_{B}$ is convergent. Hence the following propositions improve the estimate of the domain of convergence of (4.5. 7).

Proposition 4. 5. 3. Let $U_{0}$ be as in Proposition 4.5.1, and set

$$
C_{5}=\max _{\mu, \nu=1, \cdots, n} \sup _{U_{0}}\left\|e_{\mu \nu}(u) \cdot 2 \sin \pi l_{\nu}\right\|_{L^{\infty}(\boldsymbol{R})}
$$

if $C_{5}<\frac{1}{n},(4.5 .14)$ is uniformly convergent in $U_{0}$.

Proof. If we denote by $E_{B}$ (resp. $P_{B}$ ) the bounded linear operator in $L^{2}(R)^{n}$ given by $\left(E_{B} f\right)(u)=E_{B}(u) f(u)$ (resp. $\left(P_{B} f\right)(u)=\int_{-\infty}^{\infty} d u^{\prime} P_{B}(u$, $\left.\left.u^{\prime}\right) \boldsymbol{f}\left(u^{\prime}\right)\right)$ for $\boldsymbol{f}(u)={ }^{t}\left(f_{1}(u), \cdots, f_{n}(u)\right) \in L^{2}(\boldsymbol{R})^{n}$. Our assumption implies that $\left\|E_{B} P_{B}\right\|_{L^{2}(\boldsymbol{R}) n}<1$. We denote by ${ }^{t} \boldsymbol{k}_{\mu}$ (resp. $\boldsymbol{j}_{\nu}$ ) the $\mu$-th row vector (resp. the $\nu$-th column vector) of $E_{B}(u)$. Denoting by $($,$) the$ inner product in $L^{2}(\boldsymbol{R})^{n}$, we have

$$
\begin{gathered}
\left|\int_{-\infty}^{\infty} \cdots \int_{-\infty}^{\infty} \frac{d u_{1}}{2 \pi} \cdots \frac{d u_{l}}{2 \pi}\left(E_{B}\left(u_{1}\right) P_{B}\left(u_{1}, u_{2}\right) \cdots E_{B}\left(u_{l}\right)\right)_{\mu \nu}\right| \\
=\left|\left(\boldsymbol{k}_{\mu}, P_{B}\left(E_{B} P_{B}\right)^{l-1} \boldsymbol{j}_{\nu}\right)\right| \leqq C_{6}\left\|E_{B} P_{B}\right\|_{L^{2}(\boldsymbol{R})^{n}}^{l-1},
\end{gathered}
$$

where $C_{6}=\left\|\boldsymbol{k}_{\mu}\right\|_{L^{2}(\boldsymbol{R}) n}\left\|P_{B}\right\|_{L^{2}(\boldsymbol{R}) n}\left\|\boldsymbol{j}_{\nu}\right\|_{L^{2}(\boldsymbol{R}) n}$. Hence $\partial \log \tau_{B} / \partial\left(-m a_{\nu}^{-}\right) \quad(\nu=1$, $\cdots, n)$ are uniformly convergent. Likewise we can prove the convergence of the other half.

Let $U_{1}$ (resp. $U_{2}$ ) be a relatively compact open subset in $C^{n}$ (resp. 
$\left.C^{n(n-1) / 2}\right)$. We assume that

$$
\max _{\mu, \nu=1, \cdots, n} \sup _{U_{1}}\left|\operatorname{Re}\left(l_{\mu}-l_{\nu}\right)\right|<1 .
$$

For $L=\left(\delta_{\mu \nu} l_{\nu}\right)_{\mu, \nu=1, \cdots, n} \in U_{1}$ we set $l_{\nu}^{\prime}=l_{\nu}-\frac{1}{2}\left(\max _{\mu=1, \cdots, n} \operatorname{Re} l_{\mu}+\min _{\mu=1, \cdots, n} \operatorname{Re} l_{\mu}\right)$. Then (4.5.15) implies that $\left|\operatorname{Re} l_{\nu}^{\prime}\right|<\frac{1}{2}$. We set

$$
e_{\mu \nu}^{\prime}(u)=e_{\mu \nu}(u)|u|^{l_{\nu}-l_{\mu}},
$$

$$
E_{B}^{\prime}(u)=\left(e_{\mu \nu}^{\prime}(u)\right)_{\mu, \nu=1, \ldots, n},
$$

$(4.5 .18)_{B} \quad P_{B}^{\prime}\left(u, u^{\prime}\right)=\left(\begin{array}{c}2 \sin \pi l_{1} \cdot|u|^{-l_{1}^{\prime}}\left|u^{\prime}\right| u_{1}^{\prime} \\ \ddots \\ 2 \sin \pi l_{n} \cdot|u|^{-l_{n}^{\prime}\left|u^{\prime}\right| u_{n}^{\prime}}\end{array}\right) \frac{1}{u-u^{\prime}+i 0}$.

We denote by $E_{B}^{\prime}$ and $P_{B}^{\prime}$ bounded linear operators in $L^{2}(\boldsymbol{R})^{n}$ given by

$$
\left(E_{B}^{\prime} g\right)(u)=E_{B}^{\prime}(u) g(u),
$$

and

$$
\left(P_{B}^{\prime} g\right)(u)=\int_{-\infty}^{\infty} \frac{d u^{\prime}}{2 \pi} P_{B}^{\prime}\left(u, u^{\prime}\right) g\left(u^{\prime}\right)
$$

respectively. The boundedness of $P_{B}^{\prime}$ is proved in Section 2.3. We set

$$
\begin{aligned}
& C_{7}=\sup _{U_{1}}\left\|P_{B}^{\prime}\right\|_{L^{2}(\boldsymbol{R})^{n}}, \\
& C_{8}=\max _{\mu, \nu=1, \cdots, n} \sup _{U_{1} \times U_{2}} \mid \lambda_{\mu \nu} \exp \left(-\pi i\left(\frac{\varepsilon_{\mu \nu}+1}{2} l_{\mu}+\frac{\varepsilon_{\mu \nu}-1}{2} l_{\nu}\right) .\right.
\end{aligned}
$$

Now we have

$$
\begin{gathered}
\int_{-\infty}^{\infty} \cdots \int_{-\infty}^{\infty} \frac{d u_{1}}{2 \pi} \cdots \frac{d u_{l}}{2 \pi}\left(E_{B}\left(u_{1}\right) P_{B}\left(u_{1}, u_{2}\right) \cdots E_{B}\left(u_{l}\right)\right)_{\mu \nu} \\
=\left(k_{\mu}^{\prime}, P_{B}^{\prime}\left(E_{B}^{\prime} P_{B}^{\prime}\right)^{l-1} \boldsymbol{j}_{\nu}^{\prime}\right),
\end{gathered}
$$

where

$$
\begin{aligned}
{ }^{t} \boldsymbol{k}_{\mu}^{\prime} & =\left(e_{\mu 1}(u)|u|^{l_{1}}, \cdots, e_{\mu n}(u)|u|^{l_{n}}\right), \\
{ }^{t} \boldsymbol{j}_{\nu}^{\prime} & =\left(e_{1 \nu}(u)|u|^{-l_{1}}, \cdots, e_{n \nu}(u)|u|^{-l_{n}}\right) .
\end{aligned}
$$

Hence we have the following proposition.

Proposition 4. 5. 4. We assume that $C_{7} C_{8}<\frac{1}{n}$. Then (4. 5. 14) is uniformly convergent in any relatively compact open subset of $Y^{n, \boldsymbol{C}} \times U_{1} \times U_{2}$. 
Remark. The complexified Poincaré group acts on $\left(X^{\boldsymbol{C}}\right)^{n}$ by $\left(a_{1}^{+}\right.$, $\left.a_{1}^{-}, \cdots, a_{n}^{+}, a_{n}^{-}\right) \mapsto\left(c a_{1}^{+}+b^{+}, c^{-1} a_{1}^{-}+b^{-}, \cdots, c a_{n}^{+}+b^{+}, c^{-1} a_{n}^{-}+b^{-}\right)$, where $c$ $\neq 0, b^{ \pm} \in C . \quad \log \tau_{B}\left(a_{1}, \cdots, a_{n} ; L, \Lambda\right)$ is invariant under this action. This is obvious for the translation $(c=1)$. If $c \neq 1$, we can deform simultaneously the paths of integrations. Then the homogeneity of $R_{B}\left(u, u^{\prime} ; l\right)$, $R_{B}\left(c u, c u^{\prime} ; l\right)=R_{B}\left(u, u^{\prime} ; l\right)$, assures the invariance. In other words, for sufficiently small $\lambda_{\mu \nu}$ 's and $l_{\nu}$ 's $\log \tau_{B}$ is analytic at $\left(a_{1}, \cdots, a_{n}\right)$ as long as for some $\theta$ the following condition is satisfied.

$$
\operatorname{Im}\left(e^{ \pm i \theta} a_{1}^{ \pm}\right)<\operatorname{Im}\left(e^{ \pm i \theta} a_{2}^{ \pm}\right)<\cdots<\operatorname{Im}\left(e^{ \pm i \theta} a_{n}^{ \pm}\right) .
$$

In particular, if $a_{1}, \cdots, a_{n}$ are real and satisfy $a_{1}^{+}<\cdots<a_{n}^{+}, a_{1}^{-}>\cdots>a_{n}^{-}$or $a_{1}^{+}>\cdots>a_{n}^{+}, a_{1}^{-}<\cdots<a_{n}^{-}, \log \tau_{B}$ is analytic at $\left(a_{1}, \cdots, a_{n}\right)$. Also we note that $\log \tau_{B}$ is analytic if

$$
\operatorname{Im} a_{1}^{ \pm}<\cdots<\operatorname{Im} a_{\nu-1}^{ \pm}=\operatorname{Im} a_{\nu}^{ \pm}<\cdots<\operatorname{Im} a_{n}^{ \pm}
$$

except for $a_{\nu-1}^{+}=a_{\nu}^{+}$or $a_{\nu-1}^{-}=a_{\nu}^{-}$.

The infinite series expansion for the wave function $w_{B}\left(x^{*}, x\right)=$ $w_{B}\left(x^{*}, x ; a_{1}, \cdots, a_{n} ; L, \Lambda\right)$ is obtained from (A. 28). Setting

$$
\begin{aligned}
& e_{\nu}^{*}(x)=-e^{\pi i l_{\nu}}|u|^{-l_{\nu}} \theta(u) e^{-i m\left\{\left(x-a_{\nu}\right)^{-} u+\left(x-a_{\nu}\right)^{+} u-1\right\}}, \\
& e_{\nu}^{* \prime}(x)=|u|^{-l_{\nu}} \theta(-u) e^{-i m\left\{\left(x-a_{\nu}\right)^{\left.-u+\left(x-a_{\nu}\right)^{+} u-1\right\}},\right.} \\
& e_{\nu}(x)=|u|^{l_{\nu}-1} \theta(u) e^{i m\left\{\left(x-a_{\nu}\right)^{-u}+\left(x-a_{\nu}\right)^{+} u^{-1}\right\}}, \\
& e_{\nu}^{\prime}(x)=e^{-\pi i l_{\nu}}|u|^{l_{\nu}-1} \theta(-u) e^{i m\left\{\left(x-a_{\nu}\right)^{-u} u\left(x-a_{\nu}\right)^{+} u^{-1}\right\}},
\end{aligned}
$$

we have

$$
w_{B}\left(x^{*}, x\right)_{\mu \nu}=\frac{\left\langle\phi^{*(\mu)}\left(x^{*}\right) \varphi_{B}^{(1)}\left(a_{1} ; l_{1}\right) \cdots \varphi_{B}^{(n)}\left(a_{n} ; l_{n}\right) \phi^{(\nu)}(x)\right\rangle_{A}}{\left\langle\varphi_{B}^{(1)}\left(a_{1} ; l_{1}\right) \cdots \varphi_{B}^{(n)}\left(a_{n} ; l_{n}\right)\right\rangle_{A}}
$$

$$
\begin{aligned}
= & \left\langle\phi^{*(\mu)}\left(x^{*}\right) \phi^{(\nu)}(x)\right\rangle_{A} \\
& +\int \frac{d u}{2 \pi}\left(\lambda_{\mu 1} e_{1}^{*}\left(x^{*}\right), \cdots, \lambda_{\mu n} e_{n}^{*}\left(x^{*}\right)\right) \sum_{l=0}^{\infty} P_{B}\left(E_{B} P_{B}\right)^{\imath}\left(\begin{array}{c}
e_{1}(x) \lambda_{1 \nu} \\
\vdots \\
e_{n}(x) \lambda_{n \nu}
\end{array}\right) .
\end{aligned}
$$

We fix $\left(a_{1}, \cdots, a_{n}\right)$ in $Y^{n \text { Euc }}=Y^{n, \boldsymbol{C}} \cap\left(X^{\mathrm{Euc}}\right)^{n}$ and choose $(L, \Lambda)$ so that the series $\sum_{l=0}^{\infty} P_{B}\left(E_{B} P_{B}\right)^{l}$ is convergent. If $\operatorname{Im}\left(x^{*}-a_{\lambda}\right)^{ \pm}<0$ (resp. $\operatorname{Im}\left(a_{\lambda}\right.$ $\left.-x)^{ \pm}<0\right), e_{\lambda}^{*}\left(x^{*}\right)$ (resp. $e_{\lambda}(x)$ ) belongs to $L^{2}(\boldsymbol{R})$ as a function of $u$. 
Hence if $\operatorname{Im} x^{* \pm}<\operatorname{Im} a_{1}^{ \pm}$and $\operatorname{Im} a_{n}^{ \pm}<\operatorname{Im} x^{ \pm}$, (4. 5. 17) is convergent. We consider the analytic behavior of $w_{B}\left(x^{*}, x\right)$ when $\left(x^{*}, x\right) \in X^{\text {Euc }} \times X^{\text {Euc }}$.

Theorem 4. 5. 5. We set $\lambda_{(\mu)}=\left(\lambda_{\mu 1}, \cdots, \lambda_{\mu n}\right)$ and $\lambda^{(\nu)}=\left(\lambda_{1 \nu}, \cdots, \lambda_{n \nu}\right)$. The $\mu$-th row vector of $w_{B}\left(x^{*}, x\right)$ belongs to $W_{B x^{*}, a_{1}, \cdots, a_{n}}^{0, l_{1}, \ldots, l_{n}}\left(\Lambda ; \lambda_{(\mu)}\right)$ as a function of $x$. The $\nu$-th column vector of $w_{B}\left(x^{*}, x\right)$ belongs to $W_{B a_{1}, \ldots, a_{n}, x}^{* l_{1}, \ldots, l_{n}, 0}\left(\Lambda, \lambda^{(\nu)}\right)$.

Proof. We fix $x^{*}$ in $X^{\text {Euc }}$ so that $\operatorname{Im} x^{* \pm}<\operatorname{Im} a_{1}^{ \pm}$. Abbreviating $\varphi_{B}^{(\lambda)}\left(a_{\lambda} ; l_{\lambda}\right)$ to $\varphi_{B}^{(\lambda)}(\lambda=1, \cdots, n)$, we set

$$
\begin{aligned}
w_{B}^{\prime}(x)_{\mu \nu} & =\frac{\left\langle\phi^{*(\mu)}\left(x^{*}\right) \varphi_{B}^{(1)} \cdots \varphi_{B}^{(\nu-1)} \phi^{(\nu)}(x) \varphi_{B}^{(\nu)} \varphi_{B}^{(\nu+1)} \cdots \varphi_{B}^{(n)}\right\rangle_{A}}{\left\langle\varphi_{B}^{(1)} \cdots \varphi_{B}^{(n)}\right\rangle_{A}}, \\
w_{B}^{\prime \prime}(x)_{\mu \nu} & =\frac{\left\langle\phi^{*(\mu)}\left(x^{*}\right) \varphi_{B}^{(1)} \cdots \varphi_{B}^{(\nu-1)} \varphi_{B} \phi^{(\nu)}(x) \varphi_{B}^{(\nu+1)} \cdots \varphi_{B}^{(n)}\right\rangle_{A}}{\left\langle\varphi_{B}^{(1)} \cdots \varphi_{B}^{(n)}\right\rangle_{A}} .
\end{aligned}
$$

Corresponding to (A. 28) and (A. 29), $w_{B}^{\prime}(x)_{\mu \nu}$ has two analytic expressions. The first one corresponding to (A. 28) is as follows.

$(4.5 .21)\left\langle\phi^{(*)(\mu)}\left(x^{*}\right) \phi^{(\nu)}(x)\right\rangle_{A}$

$$
+\int \frac{d u}{2 \pi}\left(\lambda_{\mu 1} e_{1}^{*}\left(x^{*}\right), \cdots, \lambda_{\mu n} e_{n}^{*}\left(x^{*}\right)\right) \sum_{l=0}^{\infty} P_{B}\left(E_{B} P_{B}\right)^{l}\left(\begin{array}{c}
e_{1}(x) \lambda_{1 \nu} \\
\vdots \\
e_{\nu-1}(x) \lambda_{\nu-1 \nu} \\
e_{\nu}^{\prime}(x) \lambda_{\nu \nu} \\
\vdots \\
e_{n}^{\prime}(x) \lambda_{n \nu}
\end{array}\right) .
$$

By the same argument as for (4.5.20) we know that (4.5.21) is analytic if $\operatorname{In} a_{\nu-1}^{ \pm}<\operatorname{Im} x^{ \pm}<\operatorname{Im} a_{\nu}^{ \pm}$. Moreover the Euclidean invariance assures that (4.5.21) is analytic if $\operatorname{Im} a_{\nu-1}^{ \pm} \leqq \operatorname{Im} x^{ \pm} \leqq \operatorname{Im} a_{\nu}^{ \pm}$except for $x=a_{\nu-1}$ or $x=a_{\nu}$. (See the remark below Proposition 4.5.4.)

The second expression for

$$
w_{B}^{\prime}(x)_{\mu \nu}=\frac{\left\langle\phi^{*(\mu)}\left(x^{*}\right) \varphi_{B}^{(1)} \cdots \varphi_{B}^{(\nu-1)}\left(\phi^{(\nu)}(x) \varphi_{B}^{(\nu)}\right) \varphi_{B}^{(\nu+1)} \cdots \varphi_{B}^{(n)}\right\rangle_{A}}{\left\langle\varphi_{B}^{(1)} \cdots \varphi_{B}^{(n)}\right\rangle_{A}}
$$

which corresponds to (A. 29) is as follows.

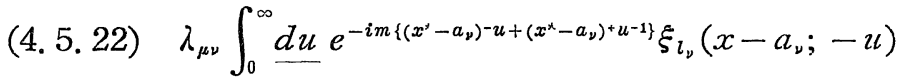

$+\int \frac{d u}{2 \pi}\left(\lambda_{\mu 1} e_{1}^{*}\left(x^{*}\right), \cdots, \lambda_{\mu n} e_{n}^{*}\left(x^{*}\right)\right) \sum_{l=0}^{\infty} P_{B}\left(E_{B} P_{B}\right)^{l}$ 


$$
\times\left(\begin{array}{c}
e_{1}\left(a_{\nu}\right) \lambda_{1 \nu} \xi_{l_{\nu}}\left(x-a_{\nu} ;-u\right) \\
\vdots \\
e_{\nu-1}\left(a_{\nu}\right) \lambda_{\nu-1} \xi_{\nu}\left(x-a_{\nu} ;-u\right) \\
0 \\
e_{\nu+1}^{\prime}\left(a_{\nu}\right) \lambda_{\nu+1} \xi_{\nu}\left(x-a_{\nu} ;-u\right) \\
\vdots \\
e_{n}^{\prime}\left(a_{\nu}\right) \lambda_{n \nu} \xi_{l_{\nu}}\left(x-a_{\nu} ;-u\right)
\end{array}\right) .
$$

The proof of coincidence of these two expressions given in the Appendix works here, for it consists simply of algebraic manipulations of bounded operators. Hence (4.5.21) and (4.5.22) give the same analytic function. From the second one we know that the local behavior of $w_{B}^{\prime}(x)_{\mu \nu}$ at $x=a_{\nu}$ is of the form

$$
\begin{aligned}
w_{B}^{\prime}(x)_{\mu \nu}=c & \cdot v_{-l+1}\left(-\left(x-a_{\nu}\right)^{-}+i 0,\left(x-a_{\nu}\right)^{+}-i 0\right)+\cdots \\
& +c^{*} \cdot v_{l}^{*}\left(-\left(x-a_{\nu}\right)^{-}+i 0,\left(x-a_{\nu}\right)^{+}-i 0\right)+\cdots .
\end{aligned}
$$

An analogous argument for $w_{B}^{\prime \prime}(x)_{\mu \nu}$ shows that $w_{B}^{\prime}(x)_{\mu \nu}$ and $w_{B}^{\prime \prime}(x)_{\mu \nu}$ coincide if $\operatorname{Im} x^{ \pm}=\operatorname{Im} a_{\nu}^{ \pm}$and $\operatorname{Re}\left( \pm x^{ \pm}\right)>\operatorname{Re}\left( \pm a_{\nu}^{ \pm}\right)$.

Now let us consider the differences

$$
\begin{aligned}
& w_{B}^{\prime}(x)_{\mu ; \nu \nu^{\prime}}=\frac{\left\langle\phi^{*(\mu)}\left(x^{*}\right) \varphi_{B}^{(1)} \cdots \varphi_{B}^{(\nu-1)} \phi^{\left(\nu^{\prime}\right)}(x) \varphi_{B}^{(\nu)} \varphi_{B}^{(\nu+1)} \cdots \varphi_{B}^{(n)}\right\rangle_{A}}{\left\langle\varphi^{(1)} \cdots \varphi^{(n)}\right\rangle_{A}}-\lambda_{\nu^{\prime} \nu} w_{B}^{\prime}(x)_{\mu \nu}, \\
& w_{B}^{\prime \prime}(x)_{\mu ; \nu^{\prime}} \underset{\operatorname{def}}{=} \frac{\left\langle\phi^{*(\mu)}\left(x^{*}\right) \varphi_{B}^{(1)} \cdots \varphi_{B}^{(\nu-1)} \varphi_{B}^{(\nu)} \phi^{\left(\nu^{\prime}\right)}(x) \varphi_{B}^{(\nu+1)} \cdots \varphi_{B}^{(n)}\right\rangle_{A}}{\left\langle\varphi_{B}^{(1)} \cdots \varphi_{B}^{(n)}\right\rangle_{A}}-\lambda_{\nu^{\prime} \nu} w_{B}^{\prime \prime}(x)_{\mu \nu},
\end{aligned}
$$

where the quotients of expectation values are defined by using (A. 29), or equivalently (A. 30).

Since we have

$$
\begin{aligned}
\operatorname{Nr}\left(\left(\phi^{\left(\nu^{\prime}\right)}(x)-\lambda_{\nu^{\prime} \nu} \phi^{(\nu)}(x)\right) \varphi_{B}^{(\nu)}\right) & =\operatorname{Nr}\left(\varphi_{B}^{(\nu)}\left(\phi^{\left(\nu^{\prime}\right)}(x)-\lambda_{\nu \nu^{\prime}} \phi^{(\nu)}(x)\right)\right) \\
& =\left(\phi^{\left(\nu^{\prime}\right)}(x)-\lambda_{\nu^{\prime} \nu} \phi^{(\nu)}(x)\right) \varphi_{B}^{(\nu)},
\end{aligned}
$$

we conclude that $w_{B}^{\prime}(x)_{\mu ; \nu \nu^{\prime}}$ and $w_{B}^{\prime \prime}(x)_{\mu ; \nu \nu^{\prime}}$ coincide and are regular at $x=a_{\nu}$. Hence all wave functions of the form

$$
\frac{\left\langle\phi^{*(\mu)}\left(x^{*}\right) \varphi_{B}^{(1)} \cdots \varphi_{B}^{(\nu-1)} \phi^{\left(\nu^{\prime}\right)}(x) \varphi_{B}^{(\nu)} \cdots \varphi_{B}^{(n)}\right\rangle_{A}}{\left\langle\varphi_{B}^{(1)} \cdots \varphi_{B}^{(n)}\right\rangle_{A}}
$$

$(\nu=1, \cdots, n+1)$ are analytic continuations of $w_{B}\left(x^{*}, x\right)_{\mu \nu}$. The local behavior of $w_{B}\left(x^{*}, x\right)_{\mu \nu}$ at $x=x^{*}$ is known from the expansion 


$$
\begin{gathered}
w_{B}\left(x^{*}, x\right)_{\mu \nu}=\frac{\left\langle\phi^{*(\mu)}\left(x^{*}\right) \phi^{(\nu)}(x) \varphi_{B}^{(1)} \cdots \varphi_{B}^{(n)}\right\rangle_{A}}{\left\langle\varphi_{B}^{(1)} \cdots \varphi_{B}^{(n)}\right\rangle_{A}} \\
=\left\langle\phi^{*(\mu)}\left(x^{*}\right) \phi^{(\nu)}(x)\right\rangle+w_{r e g}(x) \\
w_{r e g}(x)=\int \frac{d u}{2 \pi}\left(\lambda_{\mu 1} e_{1}^{*}\left(x^{*}\right), \cdots, \lambda_{\mu n} e_{n}^{*}\left(x^{*}\right)\right) \sum_{l=0}^{\infty} P_{B}\left(E_{B} P_{B}\right)^{l}\left(\begin{array}{c}
e_{1}^{\prime}(x) \lambda_{1 \nu} \\
\vdots \\
e_{n}^{\prime}(x) \lambda_{n \nu}
\end{array}\right) .
\end{gathered}
$$

Since $w_{\text {reg }}(x)$ is regular at $x=x^{*}, w_{B}\left(x^{*}, x\right)_{\mu \nu}$ has the desired property there, hence everywhere in the finite plane.

It is obvious that $w_{B}\left(x^{*}, x\right)_{\mu \nu}$ satisfy the Klein-Gordon equation. It is also easy to see that the $L^{2}$-norm of $e_{\lambda}(x)$ is bounded if $\operatorname{Im}\left(x-a_{\lambda}\right)^{ \pm}$ $>\varepsilon>0(\lambda=1, \cdots, n)$. Hence $w_{B}\left(x^{*}, x\right)_{\mu \nu}$ is bounded there. Likewise the monodromy properties and the Euclidean invariance imply the boundedness of $w_{B}\left(x^{*}, x\right)_{\mu \nu}$ as $|x| \rightarrow \infty$. Thus we have proved the first half of Theorem 4.5.5. The second half is proved similarly.

Remark 1. If we choose $(L, \Lambda)$ so that $\max _{\mu, \nu=1, \cdots, n}\left|\operatorname{Re}\left(l_{\mu}-l_{\nu}\right)\right|<1$ and that the series $\sum_{l=0}^{\infty} P_{B}^{\prime}\left(E_{B}^{\prime} P_{B}^{\prime}\right)^{l}$ is convergent, then (4.5.20) is uniformly convergent in any relatively compact open subset of

$$
\begin{aligned}
& \left\{\left(x^{*}, x, a_{1}, \cdots, a_{n}\right) \in\left(X^{c}\right)^{n+2} \mid \operatorname{Im} x^{* \pm}<\operatorname{Im} a_{\nu}^{ \pm}<\operatorname{Im} x^{ \pm}\right. \\
& \left.(\nu=1, \cdots, n), \operatorname{Im} a_{\mu}^{ \pm} \leqq \operatorname{Im} a_{\nu}^{ \pm}(1 \leqq \mu<\nu \leqq n)\right\} \times U_{1} \times U_{2} .
\end{aligned}
$$

Since we have proved the existence theorem for wave functions in Section 3. 2 , - functions and wave functions are real analytic with respect to arbitrary, distinct $x^{*}, x$ and $a_{1}, \cdots, a_{n}$ as long as $\Lambda$ is real and positive definite and $0<l_{\nu}<1(\nu=1, \cdots, n)$. For example, for $n=2$, this condition says -1 $<\lambda<1$ where $\Lambda=\left(\begin{array}{ll}1 & \lambda \\ \lambda & 1\end{array}\right)$. We note that the convergence proof in this section cannot cover this interval if $\left|a_{1}-a_{2}\right| \rightarrow 0$.

Remark 2. As mentioned above in the proof, once we have established the convergence of the infinite series, the formal arguments in Section 4.3 and Section 4.4 survive as rigorous ones. In particular, the associative law

(4. 5. 23) $\left\langle\varphi_{1} \cdots \varphi_{n}\right\rangle=\left\langle\varphi_{1} \cdots\left(\varphi_{\nu} \varphi_{\nu+1}\right) \cdots \varphi_{n}\right\rangle$,

(4. 5. 24) $\frac{\varphi_{1} \cdots \varphi_{n}}{\left\langle\varphi_{1} \cdots \varphi_{n}\right\rangle}=\varphi_{1} \cdots\left(\frac{\varphi_{\nu} \varphi_{\nu+1}}{\left\langle\varphi_{\nu} \varphi_{\nu+1}\right\rangle}\right) \cdots \varphi_{n} \mid\left\langle\varphi_{1} \cdots\left(\frac{\varphi_{\nu} \varphi_{\nu+1}}{\left\langle\varphi_{\nu} \varphi_{\nu+1}\right\rangle}\right) \cdots \varphi_{n}\right\rangle$ 
are valid as long as they are convergent, since (4.5.23) and (4.5.24) follow from changing the order in summation of $\sum_{l=0}$ trace $\left(A_{B} R_{B}\right)^{l}$ and $\sum_{\mu, \nu=1}^{n}\left\{R_{B}\left(A_{B} R_{B}\right)^{\imath}\right\}_{\mu \nu}$ respectively.

We shall list the infinite series expansions for several n-point functions in Section 4.4. In the sequel we assume that $\operatorname{Im} a_{1}^{ \pm}<\cdots<\operatorname{Im} a_{n}^{ \pm}$.

$(4.5 .25)_{B} \frac{\left\langle\varphi_{B}^{(1)} \cdots \varphi_{B}^{\left(\mu^{\prime}-1\right)} \phi^{*(\mu)}\left(x^{*}\right) \varphi_{B}^{\left(\mu^{\prime}\right)} \cdots \varphi_{B}^{\left(\nu^{\prime}-1\right)} \phi^{(\nu)}(x) \varphi_{B}^{\left.\left(\nu^{\prime}\right) \cdots \varphi_{B}^{(n)}\right\rangle_{A}}\right.}{\left\langle\varphi_{B}^{(1)} \cdots \varphi_{B}^{(n)}\right\rangle_{A}}$

$$
\begin{aligned}
& =\frac{\lambda_{\mu \nu}}{\pi} \widetilde{v}_{0}\left(-\left(x^{*}-x\right)^{-},\left(x^{*}-x\right)^{+}\right) \\
& \quad+\int \frac{d u}{2 \pi}\left(\lambda_{\mu 1} e_{1}^{* \prime}\left(x^{*}\right), \cdots, \lambda_{\mu \mu^{\prime}} e_{\mu^{\prime}}^{*}\left(x^{*}\right), \cdots\right) \sum_{l=0}^{\infty} P_{B}\left(E_{B} P_{B}\right)^{\imath}\left(\begin{array}{c}
e_{1}(x) \lambda_{1 \nu} \\
\vdots \\
e_{\nu^{\prime}}^{\prime}(x) \lambda_{\nu^{\prime} \nu} \\
\vdots
\end{array}\right) .
\end{aligned}
$$

$(4.5 .26)_{B} \quad \frac{\left\langle\varphi_{B}^{(1)} \cdots \varphi_{l_{\mu}+j}^{B *(\mu)}\left(a_{\mu} ; l_{\mu}\right) \cdots \varphi_{B}^{\left(\nu^{\prime}-1\right)} \phi^{(\nu)}(x) \varphi_{B}^{\left(\nu^{\prime}\right) \cdots} \varphi_{B}^{(n)}\right\rangle_{A}}{\left\langle\varphi_{B}^{(1)} \cdots \varphi_{B}^{(n)}\right\rangle_{A}}$

$$
\begin{aligned}
= & \frac{e^{2 \pi i l_{\mu}} \lambda_{\mu \nu}}{\pi} \widetilde{v}_{-l_{\mu}-j}\left(-\left(a_{\mu}-x\right)^{-},\left(a_{\mu}-x\right)^{+}\right) \\
& +\int \frac{d u}{2 \pi}\left(\lambda_{\mu 1} e_{1}^{* \prime}\left(a_{\mu}\right)(0+i u)^{l_{\mu}+j}, \cdots, 0, \lambda_{\mu \mu+1} e_{\mu+1}^{*}\left(a_{\mu}\right)(0+i u)^{l_{\mu}+j}, \cdots\right) \\
& \times \sum_{l=0}^{\infty} P_{B}\left(E_{B} P_{B}\right)^{l}\left(\begin{array}{c}
e_{1}(x) \lambda_{1 \nu} \\
\vdots \\
e_{\nu^{\prime}}^{\prime}\left(\begin{array}{c}
x \\
\vdots
\end{array} \lambda_{\nu^{\prime} \nu}\right. \\
\vdots
\end{array}\right) .
\end{aligned}
$$

$(4.5 .27)_{B} \quad \frac{\left\langle\varphi_{B}^{(1)} \cdots \varphi_{B}^{\left(\mu^{\prime}-1\right)} \phi^{*(\mu)}\left(x^{*}\right) \varphi_{B}^{\left(\mu^{\prime}\right)} \cdots \varphi_{-l_{\nu}+k}^{B(\nu)}\left(a_{\nu}: l_{\nu}\right) \cdots \varphi_{B}^{(n)}\right\rangle_{A}}{\left\langle\varphi_{B}^{(1)} \cdots \varphi_{B}^{(n)}\right\rangle_{A}}$

$$
\begin{aligned}
= & \frac{e^{\pi i\left(-l_{\nu}+k\right)} \lambda_{\mu \nu}}{\pi} \widetilde{v}_{l_{\nu}-k}\left(-\left(x^{*}-a_{\nu}\right)^{-},\left(x^{*}-a_{\nu}\right)^{+}\right) \\
& +\int \frac{d u}{2 \pi}\left(\lambda_{\mu 1} e_{1}^{* \prime}\left(x^{*}\right), \cdots, \lambda_{\mu \mu^{\prime}} e_{\mu^{\prime}}^{*}\left(x^{*}\right), \cdots\right) \\
& \times \sum_{l=0}^{\infty} P_{B}\left(E_{B} P_{B}\right)^{\imath}\left(\begin{array}{c}
e_{1}\left(a_{\nu}\right)(0-i u)^{-l_{\nu}+k} \lambda_{1 \nu} \\
\vdots \\
0 \\
e_{\nu+1}^{\prime}\left(a_{\nu}\right)(0-i u)^{-l_{\nu}+k} \lambda_{\nu+1} \\
\vdots
\end{array}\right) .
\end{aligned}
$$


$(4.5 .28)_{B} \quad \frac{\left\langle\varphi_{B}^{(1)} \cdots \varphi_{l_{\mu}+j}^{B *(\mu)}\left(a_{\mu} ; l_{\mu}\right) \cdots \varphi_{-l_{\nu+k}}^{B(\nu)}\left(a_{\nu} ; l_{\nu}\right) \cdots \varphi_{B}^{(n)}\right\rangle_{A}}{\left\langle\varphi_{B}^{(1)} \cdots \varphi_{B}^{(n)}\right\rangle_{A}}$

$$
\begin{aligned}
= & \frac{e^{2 \pi i l_{\mu}} e^{-\pi i\left(l_{\nu}-k\right)} \lambda_{\mu \nu}}{\pi} \widetilde{v}_{-l_{\mu}+l_{\nu}-j-k}\left(-\left(a_{\mu}-a_{\nu}\right)^{-},\left(a_{\mu}-a_{\nu}\right)^{+}\right) \\
& +\int \frac{d u}{2 \pi}\left(\lambda_{\mu 1} e_{1}^{* \prime}\left(a_{\mu}\right)(0+i u)^{l_{\mu}+j}, \cdots, 0, \lambda_{\mu \mu+1} e_{\mu+1}^{*}\left(a_{\mu}\right)(0+i u)^{l_{\mu}+j}, \cdots\right) \\
& \times \sum_{l=0}^{\infty} P_{B}\left(E_{B} P_{B}\right)^{l}\left(\begin{array}{c}
e_{1}\left(a_{\nu}\right)(0-i u)^{-l_{\nu}+k} \lambda_{1 \nu} \\
\vdots \\
0 \\
e_{\nu+1}^{\prime}\left(a_{\nu}\right)(0-i u)^{-l_{\nu}+k} \lambda_{\nu+1} \\
\vdots
\end{array}\right) .
\end{aligned}
$$

$(4.5 .29)_{B} \quad \frac{\left\langle\varphi_{B}^{(1)} \cdots \varphi_{B}^{(\nu-1)} \varphi_{B l_{\nu}+j,-l_{\nu}+k}^{*}\left(a_{\nu} ; l_{\nu}\right) \varphi_{B}^{(\nu)} \cdots \varphi_{B}^{(n)}\right\rangle_{A}}{\left\langle\varphi_{B}^{(1)} \cdots \varphi_{B}^{(n)}\right\rangle_{A}}$

$$
\begin{gathered}
=\int \frac{d u}{2 \pi}\left(\lambda_{\nu 1} e_{1}^{* \prime}\left(a_{\nu}\right)(0+i u)^{l_{\nu}+j}, \cdots, 0, \lambda_{\nu \nu+1} e_{\nu+1}^{*}\left(a_{\nu}\right)(0+i u)^{l_{\nu}+j}, \cdots\right) \\
\quad \times \sum_{l=0}^{\infty} P_{B}\left(E_{B} P_{B}\right)^{\imath}\left(\begin{array}{c}
e_{1}\left(a_{\nu}\right)(0-i u)^{-l_{\nu}+k} \lambda_{1 \nu} \\
\vdots \\
0 \\
e_{\nu+1}^{\prime}\left(a_{\nu}\right)(0-i u)^{-l_{\nu}+k} \lambda_{\nu+1} \\
\vdots
\end{array}\right) .
\end{gathered}
$$

Now we abbreviate $\varphi_{F}^{(\nu)}\left(a_{\nu} ; l_{\nu}\right)$ to $\varphi_{F}^{(\nu)}(\nu=1, \cdots, n)$. we set

(4. 5. 5) $)_{F} \quad E_{F}(u)=\left(-\varepsilon_{\mu \nu} e_{\mu \nu}(u)\right)_{\mu, \nu=1, \cdots, n}$,

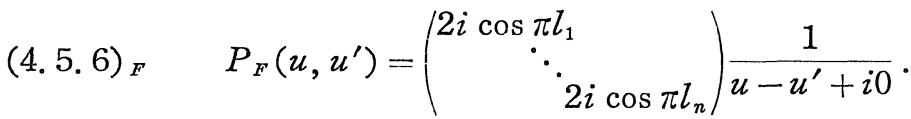

Denoting by $\varepsilon$ and $\varepsilon^{*}$ the sign + or - , we have

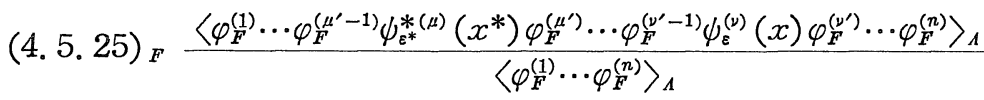

$$
\begin{aligned}
= & \frac{e^{\varepsilon^{\gamma} \pi i} e^{\varepsilon \pi i / 2} \lambda_{\mu \nu}}{\pi} \widetilde{v}_{-\left(e^{*}+\varepsilon\right) \frac{1}{2}}\left(-\left(x^{*}-x\right)^{-},\left(x^{*}-x\right)^{+}\right) \\
& +\int \frac{d u}{2 \pi}\left(-\lambda_{\mu 1} e_{1}^{* \prime}\left(x^{*}\right)(0+i u)^{\varepsilon^{* \frac{1}{2}}}, \cdots, \lambda_{\mu \mu^{\prime}} e_{\mu^{\prime}}^{*}\left(x^{*}\right)(0+i u)^{\varepsilon^{* \frac{1}{2}}}, \cdots\right) \\
& \times \sum_{l=0}^{\infty} P_{F}\left(E_{F} P_{F}\right)^{l}\left(\begin{array}{c}
e_{1}(x)(0-i u)^{\varepsilon \frac{1}{2}} \lambda_{1 \nu} \\
\vdots \\
-e_{\nu^{\prime}}^{\prime}(x)(0-i u)^{\varepsilon \frac{1}{2}} \lambda_{\nu^{\prime} \nu} \\
\vdots
\end{array}\right) .
\end{aligned}
$$


932

Mikio Sato, Tetsuji Miwa and Michio Jimbo

$(4.5 .26)_{F} \quad \frac{\left\langle\varphi_{F}^{(1)} \cdots \varphi_{l_{\mu}+j}^{F^{*}(\mu)}\left(a_{\mu} ; l_{\mu}\right) \cdots \varphi_{F}^{\left(\nu^{\prime}-1\right)} \psi_{\varepsilon}^{(\nu)}(x) \varphi_{F}^{\left(\nu^{\prime}\right)} \cdots \varphi_{F}^{(n)}\right\rangle_{A}}{\left\langle\varphi_{F}^{(1)} \cdots \varphi_{F}^{(n)}\right\rangle_{A}}$

$$
\begin{aligned}
= & \frac{e^{2 \pi i l_{\mu}} e^{\varepsilon \pi i / 2} \lambda_{\mu \nu}}{\pi} \widetilde{v}_{-\left(l_{\mu}+j+\varepsilon \frac{1}{2}\right)}\left(-\left(a_{\mu}-x\right)^{-},\left(a_{\mu}-x\right)^{+j}\right) \\
& +\int \frac{d u}{2 \pi}\left(-\lambda_{\mu 1} e_{1}^{* \prime}\left(a_{\mu}\right)(0+i u)^{l_{\mu}+j}, \cdots, 0\right. \\
& \left.\lambda_{\mu \mu+1} e_{\mu+1}^{*}\left(a_{\mu}\right)(0+i u)^{l_{\mu}+j}, \cdots\right) \\
& \times \sum_{l=0}^{\infty} P_{F}\left(E_{F} P_{F}\right)^{l}\left(\begin{array}{c}
e_{1}(x)(0-i u)^{\varepsilon \frac{1}{2}} \lambda_{1 \nu} \\
\vdots \\
-e_{\nu^{\prime}}^{\prime}(x)(0-i u)^{\varepsilon \frac{1}{2}} \lambda_{\nu^{\prime} \nu}
\end{array}\right) .
\end{aligned}
$$

$(4.5 .27)_{F} \quad \frac{\left\langle\varphi_{F}^{(1)} \cdots \varphi_{F}^{\left(\mu^{\prime}-1\right)} \psi_{\varepsilon^{*}}^{*(\mu)}\left(x^{*}\right) \varphi_{F}^{\left(\mu^{\prime}\right)} \cdots \varphi_{-l_{\nu}+k}^{F}\left(a_{\nu} ; l_{\nu}\right) \cdots \varphi_{F}^{(n)}\right\rangle_{A}}{\left\langle\varphi_{F}^{(1)} \cdots \varphi_{F}^{(n)}\right\rangle_{A}}$

$$
\begin{aligned}
= & \frac{e^{\varepsilon^{*} \pi i} e^{-\pi i\left(l_{\nu}-k\right)} \lambda_{\mu \nu}}{\pi} \widetilde{v}_{-\left(\varepsilon^{\left.* \frac{1}{2}-l_{\nu}+k\right)}\right.}\left(-\left(x^{*}-a_{\nu}\right)^{-},\left(x^{*}-a_{\nu}\right)^{+}\right) \\
& +\int \frac{d u}{2 \pi}\left(-\lambda_{\mu 1} e_{1}^{* \prime}\left(x^{*}\right)(0+i u)^{\varepsilon^{* \frac{1}{2}}}, \cdots, \lambda_{\mu \mu^{\prime}} e_{\mu^{\prime}}^{*}\left(x^{*}\right)(0+i u)^{\varepsilon^{* \frac{1}{2}}}, \cdots\right)
\end{aligned}
$$

$$
\times \sum_{l=0}^{\infty} P_{F}\left(E_{F} P_{F}\right)^{l}\left(\begin{array}{c}
e_{1}\left(a_{\nu}\right)(0-i u)^{-l_{\nu}+k} \lambda_{1 \nu} \\
\vdots \\
0 \\
-e_{\nu+1}^{\prime}\left(a_{\nu}\right)(0-i u)^{-l_{\nu}+k} \lambda_{\nu+1} \\
\vdots
\end{array}\right) .
$$

$(4.5 .28)_{F} \quad \frac{\left\langle\varphi_{F}^{(1)} \cdots \varphi_{l_{\mu}+j}^{F *(\mu)}\left(a_{\mu} ; l_{\mu}\right) \cdots \varphi_{-l_{\nu}+k}^{F(\nu)}\left(a_{\nu} ; l_{\nu}\right) \cdots \varphi_{F}^{(n)}\right\rangle_{A}}{\left\langle\varphi_{F}^{(1)} \cdots \varphi_{F}^{(n)}\right\rangle_{A}}$

$$
\begin{aligned}
= & \frac{e^{2 \pi i l_{\mu}} e^{\pi i\left(l_{\nu}-k\right)} \lambda_{\mu \nu}}{\pi} \widetilde{v}_{-l_{\mu}+l_{\nu}-j-k}\left(-\left(a_{\mu}-a_{\nu}\right)^{-},\left(a_{\mu}-a_{\nu}\right)^{+}\right) \\
& +\int \frac{d u}{2 \pi}\left(-\lambda_{\mu 1} e_{1}^{* \prime}\left(a_{\mu}\right)(0+i u)^{l_{\mu}+j}, \cdots, 0,\right. \\
& \left.\lambda_{\mu \mu+1} e_{\mu+1}^{*}\left(a_{\mu}\right)(0+i u)^{l_{\mu}+j}, \cdots\right) \\
& \times \sum_{l=0}^{\infty} P_{F}\left(E_{F} P_{F}\right)^{l}\left(\begin{array}{c}
e_{1}\left(a_{\nu}\right)(0-i u)^{-l_{\nu}+k} \lambda_{1 \nu} \\
\vdots \\
0 \\
-e_{\nu+1}^{\prime}\left(a_{\nu}\right)(0-i u)^{-l_{\nu}+k} \lambda_{\nu+1} \\
\vdots
\end{array}\right) .
\end{aligned}
$$


$(4.5 .29)_{F} \quad \frac{\left.\left\langle\varphi_{F}^{(1)} \cdots \varphi_{F}^{(\nu-1)} \varphi_{F l_{\nu}+j,-l_{\nu}+k}^{*\left(a_{\nu}\right.} ; l_{\nu}\right) \varphi_{F}^{(\nu)} \cdots \varphi_{F}^{(n)}\right\rangle_{A}}{\left\langle\varphi_{F}^{(1)} \cdots \varphi_{F}^{(n)}\right\rangle_{A}}$

$$
\begin{gathered}
=\int \frac{d u}{2 \pi}\left(-\lambda_{\nu 1} e_{1}^{* \prime}\left(a_{\nu}\right)(0+i u)^{l_{\nu}+j}, \cdots, 0, \lambda_{\nu \nu+1} e_{\nu+1}^{*}\left(a_{\nu}\right)(0+i u)^{l_{\nu}+j}, \cdots\right) \\
\quad \times \sum_{l=0}^{\infty} P_{F}\left(E_{F} P_{F}\right)^{l}\left(\begin{array}{c}
e_{1}\left(a_{\nu}\right)(0-i u)^{-l_{\nu}+k} \lambda_{1 \nu} \\
\vdots \\
0 \\
-e_{\nu+1}^{\prime}\left(a_{\nu}\right)(0-i u)^{-l_{\nu}+k} \lambda_{\nu+1} \\
\vdots
\end{array}\right)
\end{gathered}
$$

Remark 1. The identities (4.4.18)-(4.4.20) are directly checked from the above formulas.

Remark 2. If we set $\lambda_{\mu \nu}=1 \quad(\mu, \nu=1, \cdots, n)$, the above formulas give corresponding ones for $\tau_{B}(L), w_{B}\left(x^{*}, x ; L\right)$ and so on.

Example (2-point functions). Let us carry out the calculations for 2 -point functions in more detail. We set

$$
A=\left(\begin{array}{ll}
a_{1} & \\
& a_{2}
\end{array}\right), L=\left(\begin{array}{ll}
l_{1} & \\
& l_{2}
\end{array}\right), \Lambda=\left(\begin{array}{ll}
1 & \lambda \\
\lambda & 1
\end{array}\right)
$$

and $\quad t=2 m \sqrt{-\left(a_{1}^{-}-a_{2}^{-}\right)\left(a_{1}^{+}-a_{2}^{+}\right)}$.

Writing down the formula (4.5.7) in this case, we have

$$
\log \tau_{B}(L, \Lambda)=\sum_{k=1}^{\infty} \frac{(2 \tilde{\lambda})^{2 k}}{k} e_{l_{1}-l_{2}}^{(2 k)}(t)
$$

(4.5.31) $\quad e_{l}^{(k)}(t)=\int_{0}^{\infty} \cdots \int \frac{d u_{1}}{2 \pi} \cdots \frac{d u_{k}}{2 \pi}$

$$
\begin{gathered}
\times e^{-(t / 2)\left(u_{1}+u_{1}^{-1}+\cdots+u_{k}+u_{k}^{-1}\right)} \frac{1}{u_{1}+u_{2}} \frac{1}{u_{2}+u_{3}} \cdots \frac{1}{u_{k}+u_{1}} \\
\times u_{1}^{l} u_{2}^{-l} u_{3}^{l} \cdots u_{k}^{\mp l}
\end{gathered}
$$

and the parameter $\tilde{\lambda}$ is given by

$$
\tilde{\lambda}=\lambda \sqrt{\sin \pi l_{1} \cdot \sin \pi l_{2}} .
$$

In (4. 5. 31) $u_{k}^{\mp l}$ signifies $u_{k}^{-l}$ for even $k$ and $u_{k}^{l}$ for odd $k$. Expressions for the expectation values among the $\varphi^{B}$-fields are obtained by applying 
$(4.5 .23)_{B},(4.5 .24)_{B}$. For $k=0,1,2, \cdots$ we set

(4.5.33) $f_{l}^{(k)}(t)=\int_{0}^{\infty} \cdots \int \frac{d u_{1}}{2 \pi} \cdots \frac{d u_{k}}{2 \pi}$

$$
\begin{aligned}
& \times \exp \left(-\frac{t}{2}\left(u_{1}+u_{1}^{-1}+\cdots+u_{k}+u_{k}^{-1}\right)\right) \\
& \times \frac{1}{u_{1}+u_{2}} \frac{1}{u_{2}+u_{3}} \cdots \frac{1}{u_{k-1}+u_{k}} u_{1}^{l} u_{2}^{-l} u_{3}^{l} \cdots u_{k}^{\mp l}, \\
& g_{l}^{(k)}(t)=\int_{0}^{c} \cdots \int \frac{d u_{1}}{2 \pi} \cdots \frac{d u_{k}}{2 \pi} \\
& \times \exp \left(-\frac{t}{2}\left(u_{1}+u_{1}^{-1}+\cdots+u_{k}+u_{k}^{-1}\right)\right) \\
& \times \frac{1}{u_{1}+u_{2}} \frac{1}{u_{2}+u_{3}} \cdots \frac{1}{u_{k-1}+u_{k}} \frac{1}{u_{k}} u_{1}^{l} u_{2}^{-l} u_{3}^{l} \cdots u_{k}^{\mp l} .
\end{aligned}
$$

It is easy to see that $g_{l}^{(2 k+1)}(t)=g_{-l}^{(2 k+1)}(t), \quad f_{l+1}^{(1)}(t)=g_{l}^{(1)}(t)=K_{l}(t) / \pi$.

Set further

$$
\begin{aligned}
& f_{l}(t ; \tilde{\lambda})=\sum_{k=0}^{\infty}(2 \tilde{\lambda})^{2 k+1} f_{l}^{(2 k+1)}(t), \\
& g_{l}(t ; \tilde{\lambda})=\sum_{k=0}^{\infty}(2 \tilde{\lambda})^{2 k} g_{l}^{(2 k)}(t), \\
& h_{l}(t ; \tilde{\lambda})=\sum_{k=0}^{\infty}(2 \tilde{\lambda})^{2 k+1} g_{l}^{(2 k+1)}(t)=h_{-l}(t ; \tilde{\lambda}) .
\end{aligned}
$$

Notice that $f_{l}, h_{l} \rightarrow 0, g_{l} \rightarrow 1$ as $t \rightarrow \infty$. We have then

$$
\begin{aligned}
& \left\langle\varphi_{l_{1}}^{B^{*}(1)}\left(a_{1} ; l_{1}\right) \varphi_{-l_{2}+1}^{B(2)}\left(a_{2} ; l_{2}\right)\right\rangle_{\Lambda} / \tau_{B}(L, \Lambda) \\
& =-i c \varepsilon e^{-i \theta\left(l_{1}-l_{2}+1\right)} f_{l_{1}-l_{2}}(t ; \tilde{\lambda}) / 2, \\
& \left\langle\varphi_{-l_{1}+1}^{B(1)}\left(a_{1} ; l_{1}\right) \varphi_{l_{2}}^{B^{*}(2)}\left(a_{2} ; l_{2}\right)\right\rangle_{\Lambda} / \tau_{B}(L, \Lambda) \\
& =i c \varepsilon^{-1} e^{i \theta\left(l_{1}-l_{2}-1\right)} f_{-l_{1}+l_{2}}(t ; \tilde{\lambda}) / 2,
\end{aligned}
$$

(4. 5. 36) $\left\langle\varphi_{B l_{1},-l_{1}}^{*(1)}\left(a_{1} ; l_{1}\right) \varphi_{B}^{(2)}\left(a_{2} ; l_{2}\right)\right\rangle_{A} / \tau_{B}(L, \Lambda)$

$$
\begin{array}{r}
=\left(1-g_{l_{1}-l_{2}}(t ; \tilde{\lambda})\right) / 2 \sin \pi l_{1}, \\
\left\langle\varphi_{B}^{(1)}\left(a_{1} ; l_{1}\right) \varphi_{B l_{2},-l_{2}}^{*(2)}\left(a_{2} ; l_{2}\right)\right\rangle_{A} / \tau_{B}(L, \Lambda) \\
=\left(1-g_{-l_{1}+l_{2}}(t ; \tilde{\lambda})\right) / 2 \sin \pi l_{2},
\end{array}
$$


$(4.5 .37)$

$$
\begin{aligned}
& \left\langle\varphi_{l_{1}}^{B+(1)}\left(a_{1} ; l_{1}\right) \varphi_{-l_{2}}^{B(2)}\left(a_{2} ; l_{2}\right)\right\rangle_{A} / \tau_{B}(L, \Lambda) \\
& =c \varepsilon e^{-i \theta\left(l_{1}-l_{2}\right)} h_{l_{1}-l_{2}}(t ; \tilde{\lambda}) / 2, \\
& \left\langle\varphi_{-l_{1}}^{B(1)}\left(a_{1} ; l_{1}\right) \varphi_{l_{2}}^{B *(2)}\left(a_{2} ; l_{2}\right)\right\rangle_{\Lambda} / \tau_{B}(L, \Lambda) \\
& =c \varepsilon^{-1} e^{i \theta\left(l_{1}-l_{2}\right)} h_{l_{1}-l_{2}}(t ; \tilde{\lambda}) / 2,
\end{aligned}
$$

where the left hand sides are identified with their Euclidean continuations to $-\left(a_{1}^{-}-a_{2}^{-}\right)=t c^{i(\theta+\pi / 2)} / 2 m, a_{1}^{+}-a_{2}^{+}=t e^{-i(\theta-\pi / 2)} / 2 m$, and $\varepsilon=e^{(\pi i / 2)\left(l_{1}+l_{2}\right)}$, $c^{-1}=\sqrt{\sin \pi l_{1} \sin \pi l_{2}}$. From (4.4.35), the coefficients $F, G$ of the holonomic system (4.3.22) for $w\left(L-\frac{1}{2} ; \Lambda\right)$ are given by

$$
\begin{aligned}
F & =F\left(l_{1}-\frac{1}{2}, l_{2}-\frac{1}{2} ; \lambda\right) \\
& =-\left(\begin{array}{cc}
l_{1}-\frac{1}{2} & \frac{1}{2} c^{\prime} \varepsilon e^{-i\left(l_{1}-l_{2}\right) \theta} t f_{l_{1}-l_{2}}(t ; \tilde{\lambda}) \\
\frac{1}{2} c^{\prime-1} \varepsilon^{-1} e^{i\left(l_{1}-l_{2}\right) \theta} t f_{-l_{1}+l_{2}}(t ; \tilde{\lambda}) & l_{2}-\frac{1}{2}
\end{array}\right) \\
G & =G\left(\begin{array}{cc}
\left.l_{1}-\frac{1}{2}, l_{2}-\frac{1}{2} ; \lambda\right) & \varepsilon e^{-i\left(l_{1}-l_{2}\right) \theta} h_{l_{1}-l_{2}}(t ; \tilde{\lambda})
\end{array}\right) \\
& =c\left(\begin{array}{cc}
c^{\prime-1} g_{-l_{1}+l_{2}}(t ; \tilde{\lambda}) & c^{\prime} g_{l_{1}-l_{2}}(t ; \tilde{\lambda}) \\
\varepsilon^{-1} e^{i\left(l_{1}-l_{2}\right) \theta} h_{l_{1}-l_{2}}(t ; \tilde{\lambda}) &
\end{array}\right.
\end{aligned}
$$

with $c^{\prime}=\sqrt{\sin \pi l_{1} / \sin \pi l_{2}}$.

Comparing (4.5.38) with (3.3.42), we have

(4. 5. 39) $f_{ \pm l}(t ; \hat{\lambda})=-\frac{1}{t}\left(c^{\prime} \kappa_{l}(t ; \tilde{\lambda})\right) \pm 1\left(t \frac{d \psi_{l}(t ; \tilde{\lambda})}{d t} \mp l \tanh \psi_{l}(t ; \hat{\lambda})\right)$,

$$
\begin{aligned}
& g_{+l}(t: \tilde{\lambda})=\left(c^{\prime} \kappa_{l}(t ; \tilde{\lambda})\right)^{ \pm 1} \cosh \psi_{l}(t ; \tilde{\lambda}), \\
& h_{l}(t: \tilde{\lambda})=\sinh \psi_{l}(t: \tilde{\lambda})
\end{aligned}
$$

where $\kappa=\kappa_{l}(t: \tilde{\lambda}), \psi_{l}=\psi_{l}(t ; \tilde{\lambda})$ satisfy

(4. 5. 40) $\quad t \frac{d}{d t} \log \kappa=l \cdot \tanh ^{2} \psi$

$$
\frac{d}{d t}\left(t \frac{d \psi}{d t}\right)=\frac{l^{2}}{t} \tanh \psi\left(1-\tanh ^{2} \psi\right)+\frac{t}{2} \sinh 2 \psi
$$

In particular if $l=l_{1}-l_{2}=0$, then $\psi=\psi_{0}(t ; \tilde{\lambda})$ satisfies 


$$
\frac{d^{2} \psi}{d t^{2}}+\frac{1}{t} \frac{d \psi}{d t}=\frac{1}{2} \sinh 2 \psi
$$

Regarding the asymptotic behavior $\psi_{0}(t ; \tilde{\lambda}) \sim \frac{2 \tilde{\lambda}}{\pi} K_{0}(t) \quad(t \rightarrow \infty)$ we conclude that our $\psi_{0}(t ; \tilde{\lambda})$ coincides with $\psi\left(t ; 0, \frac{\tilde{\lambda}}{\pi}\right)$ in Reference [12].

The formula (3.3.57) for $d \log \tau_{B}$ combined with (4.5.38) gives

$$
\text { (4. 5. 42) } d \log \tau_{B}=-\frac{1}{2}\left(t\left(\left(\frac{d \psi}{d t}\right)^{2}-\sinh ^{2} \psi\right)-\frac{1}{t} l^{2} \tanh ^{2} \psi\right) d t
$$

or along with the boundary conditions $\tau_{B} \rightarrow 1, \psi \rightarrow 0 \quad(t \rightarrow \infty)$,

$$
\text { (4. 5. 43) } \tau_{B}=\exp \left\{-\frac{1}{2} \int_{\infty}^{t} d s\left(s\left(\left(\frac{d \psi}{d s}\right)^{2}-\sinh ^{2} \psi\right)-\frac{l^{2}}{s} \tanh ^{2} \psi\right)\right\}
$$

where $\psi=\psi_{l}(t ; \tilde{\lambda})$.

Finally we note that in the case of equal exponents $l_{1}=l_{2}$, the matrices $F\left(l_{1}-\frac{1}{2} ; \lambda\right)=F\left(l_{1}-\frac{1}{2}, l_{1}-\frac{1}{2} ; \lambda\right)$ and $G\left(l_{1}-\frac{1}{2} ; \lambda\right)=G\left(l_{1}-\frac{1}{2}\right.$, $\left.l_{1}-\frac{1}{2} ; \lambda\right)$ depends essentially on the combination $\tilde{\lambda}=\lambda \sin \pi l_{1}$ as follows.

$$
\text { (4.5.44) } \begin{aligned}
F\left(l_{1}-\frac{1}{2} ; \lambda\right) & =\left(\begin{array}{l}
1 \\
e^{-\pi i\left(l_{1}-\frac{1}{2}\right)}
\end{array}\right) F\left(0 ; \lambda \sin \pi l_{1}\right)\left(\begin{array}{l}
1 \\
e^{\pi i\left(l_{1}-\frac{1}{2}\right)}
\end{array}\right)-\left(l-\frac{1}{2}\right) \\
G\left(l_{1}-\frac{1}{2} ; \lambda\right) & =\frac{1}{\sin \pi l}\left(e^{-\pi i\left(l_{1}-\frac{1}{2}\right)}\right) G\left(0 ; \lambda \sin \pi l_{1}\right)\left(\begin{array}{l}
1 \\
e^{\pi i\left(l_{1}-\frac{1}{2}\right)}
\end{array}\right)
\end{aligned}
$$

where

$$
\text { (4.5.45) } \begin{aligned}
F(0 ; \lambda) & =-\left({ }_{i}-i\right) \frac{1}{2} t \frac{d \psi_{0}}{d t}(t ; \lambda), \\
G(0 ; \lambda) & =e^{-2 H(0 ; \lambda)}, \quad H(0 ; \lambda)=\left({ }_{i}-i\right) \frac{1}{2} \psi_{0}(t ; \lambda) .
\end{aligned}
$$

So far, we have considered vacuum expectation values. Now we study the product $\varphi_{B}\left(a_{1} ; l_{1}\right) \cdots \varphi_{B}\left(a_{n} ; l_{n}\right)$ itself. From (A. 25) we have

$$
\begin{aligned}
& \text { (4. 5. 46) } \operatorname{Nr}_{A}\left(\varphi_{B}^{(1)}\left(a_{1} ; l_{1}\right) \cdots \varphi_{B}^{(n)}\left(a_{n} ; l_{n}\right)\right) \\
& =\left\langle\varphi_{B}^{(1)}\left(a_{1} ; l_{1}\right) \cdots \varphi_{B}^{(n)}\left(a_{n} ; l_{n}\right)\right\rangle_{A} e^{\hat{\rho}_{B} / 2} \\
& \hat{\rho}_{B}=2 \iint d u d u^{\prime} \sum_{\mu, \nu=1}^{n} \widehat{R}^{(\mu \nu)}\left(u, u^{\prime}\right) \phi^{(\mu)}(u) \phi^{*(\nu)}\left(u^{\prime}\right)
\end{aligned}
$$




$$
\begin{aligned}
& \widehat{R}_{B}\left(u, u^{\prime}\right)=\left(\widehat{R}^{(\mu \nu)}\left(u, u^{\prime}\right)\right)=\sum_{l=0}^{\infty} \int \cdots \int \underline{d u_{1}} \cdots \underline{d u_{2 l}} \\
& \quad \times R_{B}\left(u, u_{1}\right) A_{B}\left(u_{1}, u_{2}\right) R_{B}\left(u_{2}, u_{3}\right) \cdots A_{B}\left(u_{2 l-1}, u_{2 l}\right) R_{B}\left(u_{2 l}, u^{\prime}\right) .
\end{aligned}
$$

If we set

(4. 5. 47) $\quad R_{B, 1}\left(u, u^{\prime}\right)$

$$
=\left(\delta_{\mu \nu} e^{-i m\left(a_{\nu}^{-} u+a_{\nu}^{+} u^{-1}\right)}(u-i 0)^{-l_{\nu}+1} \frac{-2 \sin \pi l_{\nu}}{u+u^{\prime}-i 0}\right)_{\mu, \nu=1, \ldots, n},
$$

(4. 5. 48) $\quad P_{B, 2}\left(u, u^{\prime}\right)$

$$
=\left(\delta_{\mu \nu} \frac{2 \sin \pi l_{\nu}}{u-u^{\prime}+i 0} \cdot e^{-i m\left(a_{\nu}^{-} u^{\prime}+a_{\nu}^{+} u^{\prime-1}\right)}\left(u^{\prime}-i 0\right)^{l_{\nu}}\right)_{\mu, \nu=1, \cdots, n},
$$

we have

(4. 5. 49) $\widehat{R}_{B}\left(u, u^{\prime}\right)=R_{B}\left(u, u^{\prime}\right)+\sum_{l=1}^{\infty} \int \cdots \int \frac{d u_{1}}{2 \pi} \cdots \frac{d u_{l}}{2 \pi}$

$$
\times R_{B, 1}\left(u, u_{1}\right) E_{B}\left(u_{1}\right) P_{B}\left(u_{1}, u_{2}\right) \cdots E_{B}\left(u_{l}\right) R_{B, 2}\left(u_{l}, u^{\prime}\right) .
$$

Likewise, assuming (4.5.15), we set

(4.5.50) $\quad R_{B, 1}^{\prime}\left(u, u^{\prime}\right)$

$$
=\left(\delta_{\mu \nu} e^{-i m\left(a_{\nu}^{-} u+a_{\nu}^{+} u^{-1}\right)}(u-i 0)^{-l_{\nu}+1} \frac{-2 \sin \pi l_{\nu}}{u+u^{\prime}-i 0}\left|u^{\prime}\right|^{l_{\nu}^{\prime}}\right)_{\mu, \nu=1, \ldots, n},
$$

(4.5.51) $\quad R_{B, 2}^{\prime}\left(u, u^{\prime}\right)$

$$
=\left(\delta_{\mu \nu}|u|^{-l_{\nu}^{\prime}} \frac{2 \sin \pi l_{\nu}}{u-u^{\prime}+i 0} e^{-i m\left(a_{\nu}^{-} u^{\prime}+a_{\nu}^{+} u^{\prime-1}\right)}\left(u^{\prime}-i 0\right)^{l_{\nu}}\right)_{\mu, \nu=1, \cdots, n},
$$

where $l_{\nu}^{\prime}(\nu=1, \cdots, n)$ are given in the proof of Proposition 4. 5. 4. Then we have

$$
\begin{aligned}
& \widehat{R}_{B}\left(u, u^{\prime}\right)=R_{B}\left(u, u^{\prime}\right)+\sum_{l=1}^{\infty} \int \cdots \int \frac{d u_{1}}{2 \pi} \cdots \frac{d u_{l}}{2 \pi} \\
& \times R_{B, 1}^{\prime}\left(u, u_{1}\right) E_{B}^{\prime}\left(u_{1}\right) P_{B}^{\prime}\left(u_{1}, u_{2}\right) \cdots E_{B}^{\prime}\left(u_{l}\right) R_{B, 2}^{\prime}\left(u_{l}, u^{\prime}\right) .
\end{aligned}
$$

Proposition 4. 5. 3'. Under the same condition as in Proposition 4.5.3, (4.5.49) is uniformly convergent in any relatively compact open subset of $U_{0} \times\left\{\left(u, u^{\prime}\right) \in C^{2} \mid \operatorname{Im} u>0, \operatorname{Im} u^{\prime}>0\right\}$. 
Let $\overline{Y^{n, \boldsymbol{C}}}$ denote the closure of $\quad Y^{n, \boldsymbol{C}}, \quad \overline{Y^{n, \boldsymbol{C}}}=\left\{\left(a_{1}, \cdots, a_{n}\right) \in\right.$ $\left(X^{\boldsymbol{C}}\right)^{n} \mid \operatorname{Im} a_{\mu}^{ \pm} \leqq \operatorname{Im} a_{\nu}^{ \pm}$for $\left.1 \leqq \mu<\nu \leqq n\right\}$.

Proposition 4. 5. 4'. Under the same condition as in Proposition 4. 5. 4, (4.5.52) is uniformly convergent in any relatively compact open subset of $\overline{Y^{n, \boldsymbol{C}}} \times U_{1} \times U_{2} \times\left\{\left(u, u^{\prime}\right) \in \mathbb{C}^{2} \mid \operatorname{Im} u>0\right.$, $\left.\operatorname{Im} u^{\prime}>0\right\}$. Moreover, $\widehat{R}_{B}\left(u, u^{\prime}\right)$ is of polynomial growth as $|\operatorname{Re} u|,\left|\operatorname{Re} u^{\prime}\right| \rightarrow \infty$ for fixed $\operatorname{Im} u$ and $\operatorname{Im} u^{\prime}$.

The proofs are similar to those of Propositions 4.5.3 and 4.5.4.

Remark. In Propositions 4.5. 4 and 4.5. $4^{\prime}$ the upper bound for $C_{10}$ may be chosen arbitrarily large if we choose $U_{1}$ so that

$$
C_{11}=\max _{\nu=1, \cdots, n} \sup _{U_{1}}\left|\sin \pi l_{\nu}\right|
$$

is sufficiently small. Hence if we set $\lambda_{\mu \nu}=1(\mu, \nu=1, \cdots, n)$ the original case of $\operatorname{Nr}\left(\varphi_{B}\left(a_{1} ; l_{1}\right) \cdots \varphi_{B}\left(a_{n} ; l_{n}\right)\right)$ is covered as far as $C_{11}$ is sufficiently small. We also call the reader's attention to the difference of convergence domains in Propositions 4.5.4 and 4.5.4 $4^{\prime}$ with respect to $\left(a_{1}, \cdots\right.$, $\left.a_{n}\right) . \widehat{R}_{B}\left(u, u^{\prime}\right)$ is continuous when $a_{\mu}-a_{\mu-1}$ tends to 0 , but $\tau_{B}$ is divergent. This is the same situation as in the 1-dimensional theory in Section 2.

Theorem 4. 5. 6. We assume that $a_{1}, \cdots, a_{n} \in X^{\mathrm{Min}}$ are mutually spacelike and the convergence condition of Proposition 4. 5. $4^{\prime}$ is valid with $\lambda_{\mu \nu}=1(1 \leqq \mu, \nu \leqq n)$. Then the product $\varphi_{B}\left(a_{\sigma(1)} ; l_{\sigma(1)}\right) \cdots \varphi_{B}\left(a_{\sigma(n)}\right)$; $\left.l_{\sigma(n)}\right)\left(\sigma \in S_{n}\right)$ is independent of $\sigma$. In particular the Euclidean continuation of $\left\langle\varphi_{B}\left(a_{1} ; l_{1}\right) \cdots \varphi_{B}\left(a_{n} ; l_{n}\right)\right\rangle$ is a single-valued real analytic function of $\left(a_{1}, \cdots, a_{n}\right)$ except for $a_{\mu}=a_{\nu}(1 \leqq \mu<\nu \leqq n)$.

Proof. We set

$$
\begin{aligned}
\varphi_{\sigma} & =\varphi_{B}\left(a_{\sigma(1)} ; l_{\sigma(1)}\right) \cdots \varphi_{B}\left(a_{\sigma(n)} ; l_{\sigma(n)}\right) \\
& =\left\langle\varphi_{\sigma}\right\rangle \exp \iint R_{\sigma}\left(u, u^{\prime}\right) \phi(u) \phi^{*}\left(u^{\prime}\right) .
\end{aligned}
$$

The assertion of the theorem is nothing but 


$$
\left\langle\varphi_{\sigma}\right\rangle=\left\langle\varphi_{\sigma^{\prime}}\right\rangle
$$

$$
R_{\sigma}\left(u, u^{\prime}\right)=R_{\sigma^{\prime}}\left(u, u^{\prime}\right)
$$

for $\sigma, \sigma^{\prime} \in \mathbb{S}_{n}$. Since both $\left\langle\varphi_{\sigma}\right\rangle$ and $R_{\sigma}\left(u, u^{\prime}\right)$ depend analytically on $L$, we may assume that $L$ is real.

First we assume that $n=2$. (4.5.54) follows directly from (4.5. 30). To prove (4.5.55) we may assume that $a_{1}, a_{2} \in X^{\mathrm{Min}} \cap X^{\mathrm{Euc}}$ by Lorentz covariance. For $x^{*}, x$ satisfying $\operatorname{Im} x^{* \pm}<0, \operatorname{Im} x^{ \pm}>0$, we have

$$
\begin{aligned}
& \left\langle\phi^{*}\left(x^{*}\right) \varphi_{\sigma} \phi(x)\right\rangle /\left\langle\varphi_{\sigma}\right\rangle-\left\langle\phi^{*}\left(x^{*}\right) \phi(x)\right\rangle \\
& \quad=\int_{0}^{\infty} \int_{0}^{\infty} \underline{d u} \underline{d u^{\prime}} e^{-i m\left(x^{\left.k-u+x^{*}+u^{-1}\right)} R_{\sigma}\left(-u, u^{\prime}\right) e^{i m\left(x^{-} u^{\prime}+x^{*} u^{\prime-1}\right)}\right.}
\end{aligned}
$$

From the remark below Theorem 4.5.5, we see that $\left\langle\phi^{*}\left(x^{*}\right) \varphi_{\sigma} \phi(x)\right\rangle /$ $\left\langle\varphi_{\sigma}\right\rangle$ is the canonical element $\frac{1}{\pi} v_{0}(L)$ in $W_{B, x^{2}, a_{1}, a_{2}}^{0, l_{1}, l_{2}, \text { strict }}$. Hence (4. 5.56) is independent of $\sigma$.

Setting

$$
\begin{aligned}
& x^{* \pm}=\frac{-i x^{* 2} \pm x^{* 1}}{2}, \quad x^{ \pm}=\frac{-i x^{2} \pm x^{1}}{2}, \\
& u=\frac{\omega\left(p^{1}\right)+p^{1}}{m}, \quad u^{\prime}=\frac{\omega\left(p^{1 \prime}\right)+p^{1 \prime}}{2}, \\
& \widetilde{R}_{\sigma}\left(p^{1}, p^{1 \prime}\right)=R_{\sigma}\left(-u, u^{\prime}\right),
\end{aligned}
$$

we have

$$
0=\int_{-\infty}^{\infty} \frac{d p^{1}}{2 \pi} \frac{d p^{1 \prime}}{2 \pi} e^{i x^{* 1} p^{1}} e^{-i x^{1} p^{1}} \widetilde{R}_{\sigma \sigma^{\prime}}\left(p^{1}, p^{1 \prime}\right)
$$

where

$$
\widetilde{R}_{\sigma \sigma^{\prime}}\left(p^{1}, p^{1 \prime}\right)=\frac{e^{-x^{\star 2}} \omega\left(p^{1}\right)}{\omega\left(p^{1}\right)}\left(\widetilde{R}_{\sigma}\left(p^{1}, p^{1 \prime}\right)-\widetilde{R}_{\sigma^{\prime}}\left(p^{1}, p^{1 \prime}\right)\right) \frac{e^{x^{2 \omega(}\left(p^{\prime \prime}\right)}}{\omega\left(p^{1 \prime}\right)} .
$$

We note that $\widetilde{R}_{\sigma \sigma^{\prime}}\left(p^{1}, p^{1 \prime}\right)$ is exponentially decreasing as $\left|p^{1}\right|,\left|p^{1 \prime}\right| \rightarrow \infty$. Hence we have $\widetilde{R}_{\sigma}\left(p^{1}, p^{1 \prime}\right)=\widetilde{R}_{\sigma^{\prime}}\left(p^{1}, p^{1 \prime}\right)$, or equivalently, $R_{\sigma}\left(u, u^{\prime}\right) \theta(-u)$ $\times \theta\left(u^{\prime}\right)=R_{\sigma^{\prime}}\left(u, u^{\prime}\right) \theta(-u) \theta\left(u^{\prime}\right)$. Likewise we can prove $R_{\sigma}\left(u, u^{\prime}\right) \theta(\varepsilon u)$ $\times \theta\left(\varepsilon^{\prime} u^{\prime}\right)=R_{\sigma^{\prime}}\left(u, u^{\prime}\right) \theta(\varepsilon u) \theta\left(\varepsilon^{\prime} u^{\prime}\right)$ where $\varepsilon, \varepsilon^{\prime}= \pm$. Hence we have proved the theorem in the case $n=2$.

Now we exploit (4.5.23) and (4.5.24) to prove (4.5.54) and 
(4.5.55) respectively. Since the convergence of $R_{\sigma}\left(u, u^{\prime}\right)$ is assured by Proposition 4.5.4', (4.5.55) for $n \geqq 3$ follows from the case $n=2$. As for (4.5.54) we have not yet proved the convergence of $\left\langle\varphi_{\sigma}\right\rangle$ when $a_{1}, \cdots, a_{n}$ are real. The domain of convergence for $\left\langle\varphi_{\sigma}\right\rangle$ depends on $\sigma$. From the remark below Proposition 4. 5. 4, we know that $\left\langle\varphi_{B}\left(a_{\sigma(1)} ; l_{\sigma(1)}\right)\right.$ $\left.\cdots \varphi_{B}\left(a_{\sigma(n)} ; l_{\sigma(n)}\right)\right\rangle$ is analytic when $a_{\sigma(1)}^{+}>\cdots>a_{\sigma(n)}^{+}$and $a_{\sigma(1)}^{-}<\cdots<a_{\sigma(n)}^{-}$, and that the domains of convergence for $\left\langle\varphi_{B}\left(a_{\sigma(1)} ; l_{\sigma(1)}\right) \cdots \varphi_{B}\left(a_{\sigma(n)} ; l_{\sigma(n)}\right)\right\rangle$ and for $\left\langle\varphi_{B}\left(a_{\sigma(1)} ; l_{\sigma(1)}\right) \cdots \varphi_{B}\left(a_{\sigma(j+1)} ; l_{\sigma(j+1)}\right) \varphi_{B}\left(a_{\sigma(j)} ; l_{\sigma(j)}\right) \cdots \varphi_{B}\left(a_{\sigma(n)} ; l_{\sigma(n)}\right)\right\rangle$ have a non-empty intersection. Since they coincide there, we have proved the theorem.

We shall list the commutation relations among our field operators. We assume that $a_{1}$ and $a_{2}$ are mutually spacelike. The letters $j, k$ below will denote integers.

$(4.5 .57)_{B} \quad \varphi_{B}\left(a_{1} ; l_{1}\right) \varphi_{B}\left(a_{2} ; l_{2}\right)=\varphi_{B}\left(a_{2} ; l_{2}\right) \varphi_{B}\left(a_{1} ; l_{1}\right)$.

$(4.5 .58)_{B} \quad \varphi_{B}\left(a_{1} ; l_{1}\right) \varphi_{-l_{2}+k}^{B}\left(a_{2} ; l_{2}\right)$

$$
= \begin{cases}\varphi_{-l_{2}+k}^{B}\left(a_{2} ; l_{2}\right) \varphi_{B}\left(a_{1} ; l_{1}\right), & a_{1}^{+}<a_{2}^{+} \\ e^{2 \pi i l_{1}} \varphi_{-l_{2}+k}^{B}\left(a_{2} ; l_{2}\right) \varphi_{B}\left(a_{1} ; l_{1}\right), & a_{1}^{+}>a_{2}^{+} .\end{cases}
$$

$(4.5 .59)_{B} \quad \varphi_{B}\left(a_{1} ; l_{1}\right) \varphi_{l_{2}+j}^{B *}\left(a_{2} ; l_{2}\right)$

$$
= \begin{cases}\varphi_{l_{2}+j}^{B *}\left(a_{2} ; l_{2}\right) \varphi_{B}\left(a_{1} ; l_{1}\right), & a_{1}^{+}<a_{2}^{+} \\ e^{-2 \pi i l_{1}} \varphi_{l_{2}+j}^{B *}\left(a_{2} ; l_{2}\right) \varphi_{B}\left(a_{1} ; l_{1}\right), & a_{1}^{+}>a_{2}^{+} .\end{cases}
$$

$(4.5 .60)_{B} \quad \varphi_{B}\left(a_{1} ; l_{1}\right) \varphi_{B l_{2}+j,-l_{2}+k}\left(a_{2} ; l_{2}\right)$

$$
=\varphi_{B} \underset{l_{2}+j,-l_{2}+k}{*}\left(a_{2} ; l_{2}\right) \varphi_{B}\left(a_{1} ; l_{1}\right) \text {. }
$$

$(4.5 .61)_{B} \quad \varphi_{l_{1}+j}^{B *}\left(a_{1} ; l_{1}\right) \varphi_{-l_{2}+k}^{B}\left(a_{2} ; l_{2}\right)$

$$
= \begin{cases}e^{2 \pi i l_{2}} \varphi_{-l_{2}+k}^{B}\left(a_{2} ; l_{2}\right) \varphi_{l_{1}+j}^{B *}\left(a_{1} ; l_{1}\right), & a_{1}^{+}<a_{2}^{+} \\ e^{2 \pi i l_{1}} \varphi_{-l_{2}+k}^{B}\left(a_{2} ; l_{2}\right) \varphi_{l_{1}+j}^{B *}\left(a_{1} ; l_{1}\right), & a_{1}^{+}>a_{2}^{+} .\end{cases}
$$

$(4.5 .57)_{F} \quad \varphi_{F}\left(a_{1} ; l_{1}\right) \varphi_{F}\left(a_{2} ; l_{2}\right)=\varphi_{F}\left(a_{2} ; l_{2}\right) \varphi_{F}\left(a_{1} ; l_{1}\right)$.

$(4.5 .58)_{F} \quad \varphi_{F}\left(a_{1} ; l_{1}\right) \varphi_{-l_{2}+k}^{F}\left(a_{2} ; l_{2}\right)$

$$
= \begin{cases}\varphi_{-l_{2}+k}^{F}\left(a_{2} ; l_{2}\right) \varphi_{F}\left(a_{1} ; l_{1}\right), & a_{1}^{+}<a_{2}^{+} \\ -e^{2 \pi i l_{1}} \varphi_{-l_{2}+k}^{F}\left(a_{2} ; l_{2}\right) \varphi_{F}\left(a_{1} ; l_{1}\right), & a_{1}^{+}>a_{2}^{+} .\end{cases}
$$

$(4.5 .59)_{F} \quad \varphi_{F}\left(a_{1} ; l_{1}\right) \varphi_{l_{2}+j}^{F *}\left(a_{2} ; l_{2}\right)$ 


$$
= \begin{cases}\varphi_{l_{2}+j}^{F *}\left(a_{2} ; l_{2}\right) \varphi_{F}\left(a_{1} ; l_{1}\right), & a_{1}^{+}<a_{2}^{+} \\ -e^{2 \pi i l_{2}} \varphi_{l_{2}+j}^{F^{*} *}\left(a_{2} ; l_{2}\right) \varphi_{F}\left(a_{1} ; l_{1}\right), & a_{1}^{+}>a_{2}^{+} .\end{cases}
$$

$(4.5 .60)_{F} \quad \varphi_{F}\left(a_{1} ; l_{1}\right) \varphi_{F}{ }_{l_{2}+j,-l_{2}+k}^{*}\left(a_{2} ; l_{2}\right)$

$$
=\varphi_{F} l_{2}^{*}+j,-l_{2}+k\left(a_{2} ; l_{2}\right) \varphi_{F}\left(a_{1} ; l_{1}\right)
$$

$(4.5 .61)_{F} \quad \varphi_{l_{1}+j}^{F *}\left(a_{1} ; l_{1}\right) \varphi_{-l_{2}+k}^{F}\left(a_{2} ; l_{2}\right)$

$$
= \begin{cases}e^{2 \pi i l_{2}} \varphi_{-l_{2}+k}^{F}\left(a_{2} ; l_{2}\right) \varphi_{l_{1}+j}^{F *}\left(a_{1} ; l_{1}\right), & a_{1}^{\top}<a_{2}^{+} \\ e^{2 \pi i l_{1}} \varphi_{l_{1}+j}^{F *}\left(a_{1} ; l_{1}\right) \varphi_{-l_{2}+k}^{F}\left(a_{2} ; l_{2}\right), & a_{2}^{+}<a_{1}^{+} .\end{cases}
$$

In order to prove $(4.5 .58)_{B}$ we recall the reader to (4.3.29). Combining this with $(4.5 .57)_{B}$ we have

$$
\begin{aligned}
& \varphi_{B}\left(a_{1} ; l_{1}\right) \phi(x) \varphi_{B}\left(a_{2} ; l_{2}\right) \\
= & \begin{cases}\phi(x) \varphi_{B}\left(a_{2} ; l_{2}\right) \varphi_{B}\left(a_{1} ; l_{1}\right), & a_{1}^{+}<a_{2}^{+} \\
e^{2 \pi i l_{1}} \phi(x) \varphi_{B}\left(a_{2} ; l_{2}\right) \varphi_{B}\left(a_{1} ; l_{1}\right), & a_{1}^{+}>a_{2}^{+},\end{cases}
\end{aligned}
$$

where $x$ is placed near $a_{n}$. Then the local expansion at $x=a_{2}$ yields $(4.5 .58)_{B}$. Other cases are proved similarly.

From (4.5.57) ${ }_{B}$ we have concluded that the Euclidean continuation of $\left\langle\varphi_{B}\left(a_{1} ; l_{1}\right) \cdots \varphi_{B}\left(a_{n} ; l_{n}\right)\right\rangle$ is single valued. The above commutation relations provide us with a complete information on the sheet structure of the Euclidean continuations of several $\tau$ functions and wave functions given in Section 4. 4. For example we have

$$
\begin{aligned}
& \left\langle\cdots \varphi_{B}\left(a_{1}, \bar{a}_{1} ; l_{1}\right) \varphi_{-l_{2}+k}^{B}\left(a_{2}, \bar{a}_{2} ; l_{2}\right) \cdots\right\rangle \\
= & e^{2 \pi i l_{1}}\left\langle\cdots \varphi_{B}\left(a_{1} ; l_{1}\right) \varphi_{-l_{2}+k}^{B}\left(a_{1}+e^{2 \pi i}\left(a_{2}-a_{1}\right), \bar{a}_{1}+e^{-2 \pi i}\left(\bar{a}_{2}-\bar{a}_{1}\right) ; l_{2}\right) \cdots\right\rangle
\end{aligned}
$$

for $\left|a_{1}-a_{2}\right| \ll 1$. Since $\left\langle\cdots \varphi_{B}\left(a_{1}, \bar{a}_{1} ; l_{1}\right) \varphi_{-l_{2}+k}^{B}\left(a_{2}, \bar{a}_{2} ; l_{2}\right) \cdots\right\rangle$ (resp. $\left\langle\cdots \varphi_{-l_{2}+k}^{B}\right.$ $\left.\left.\left(a_{2}, \bar{a}_{2} ; l_{2}\right) \varphi_{B}\left(a_{1}, \bar{a}_{1} ; l_{1}\right) \cdots\right\rangle\right)$ is real analytic for $\operatorname{Im}\left(a_{1}-a_{2}\right)>0$ (resp. $\left.\operatorname{Im}\left(a_{1}-a_{2}\right)<0\right),(4.5 .62)$ is a direct consequence of $(4.5 .58)_{B}$ by analytic continuation.

\section{$\S 4.6$. Neutral Theory}

In this section we shall discuss in more detail the original case of $\varphi_{B}(a), \varphi_{F}(a)$ and related fields, which are constructed on the basis of neutral free fields. As we shall see the operator theory in this case has physical content. 
The fields $\varphi_{B}(a)$ and $\varphi_{F}(a)$ are introduced in (4.1.65) and (4.2. 44) respectively. We are also interested in the fields $\varphi^{B}(a)={ }^{t}\left(\varphi_{+}^{B}(a), \varphi_{-}^{B}(a)\right)$ and $\varphi^{F}(a)$, whose norms are respectively given by (cf. (4.3.44), (4. 3.66))

$$
\begin{aligned}
& \operatorname{Nr}\left(\varphi_{ \pm}^{B}(a)\right)=\phi_{ \pm}(a) e^{\rho_{B}(a) / 2}, \\
& \phi_{ \pm}(a)=\int \underline{d u} \sqrt{0+i u^{ \pm 1}} e^{-i m\left(a-u+a^{+} u-1\right)} \phi(u),
\end{aligned}
$$

$$
\begin{aligned}
& \operatorname{Nr}\left(\varphi^{F}(a)\right)=\psi_{0}(a) e^{\rho_{F}(a) / 2}, \\
& \psi_{0}(a)=\int d u e^{-i m\left(a-u+a^{+} u^{-1}\right)} \psi(u) .
\end{aligned}
$$

As shown in (4.3.26)' and (4.3.67), they appear as the leading coefficients of the local expansions for $\phi(x) \varphi_{B}(a)$ or $\psi(x) \varphi_{F}(a)$. Clearly $\varphi_{B}(a), \varphi_{F}(a)$ and $\varphi^{F}(a)$ are Lorentz scalar, while $\varphi^{B}(a)$ transforms as a spinor.

These fields along with the auxiliary free ones obey simple commutation relations in the spacelike region of arguments; namely they either commute or anticommute. We have (cf. (3.3.26), (3.3.37) with $l=1 / 2$, $\left.l^{\prime}= \pm 1 / 2\right)$

$$
\text { (4.6.3) } \begin{aligned}
\varphi_{B}(a) \phi(x) & = \begin{cases}\phi(x) \varphi_{B}(a) & \left(x^{+}>a^{+}, x^{-}<a^{-}\right) \\
-\phi(x) \varphi_{B}(a) & \left(x^{+}<a^{+}, x^{-}>a^{-}\right),\end{cases} \\
\varphi_{ \pm}^{B}(a) \phi(x) & = \begin{cases}\phi(x) \varphi_{ \pm}^{B}(a) & \left(x^{+}>a^{+}, x^{-}<a^{-}\right) \\
-\phi(x) \varphi_{ \pm}^{B}(a) & \left(x^{+}<a^{+}, x^{-}>a^{-}\right) .\end{cases}
\end{aligned}
$$

Moreover $\varphi_{B}$ and $\varphi_{ \pm}^{B}$ satisfy

$$
\text { (4.6. 4) } \begin{aligned}
& \varphi_{B}(a) \varphi_{B}\left(a^{\prime}\right)=\varphi_{B}\left(a^{\prime}\right) \varphi_{B}(a) \quad\left(\left(a^{+}-a^{++}\right)\left(a^{-}-a^{\prime-}\right)<0\right), \\
& \varphi_{\varepsilon}^{B}(a) \varphi_{\varepsilon^{\prime}}^{B}\left(a^{\prime}\right)=-\varphi_{\varepsilon^{\prime}}^{B}\left(a^{\prime}\right) \varphi_{\varepsilon}^{B}(a) \\
& \left(\varepsilon, \varepsilon^{\prime}= \pm ;\left(a^{+}-a^{\prime+}\right)\left(a^{-}-a^{\prime-}\right)<0\right)
\end{aligned}
$$

and

(4. 6. 5) $\quad \varphi_{B}(a) \varphi_{ \pm}^{B}\left(a^{\prime}\right)= \begin{cases}\varphi_{ \pm}^{B}\left(a^{\prime}\right) \varphi_{B}(a) & \left(a^{++}>a^{+}, a^{\prime-}<a^{-}\right) \\ -\varphi_{ \pm}^{B}\left(a^{\prime}\right) \varphi_{B}(a) & \left(a^{\prime+}<a^{+}, a^{\prime-}>a^{-}\right) .\end{cases}$

Similarly we have (cf. (3.3.66), (3.3.68) with $l=0, l^{\prime}=0$ ) 
(4.6.6)

$$
\begin{aligned}
\varphi_{F}(a) \psi(x) & = \begin{cases}\psi(x) \varphi_{F}(a) & \left(x^{+}>a^{+}, x^{-}<a^{-}\right) \\
-\psi(x) \psi_{F}(a) & \left(x^{+}<a^{+}, a^{-}>a^{-}\right),\end{cases} \\
\varphi^{F}(a) \psi(x) & = \begin{cases}-\psi(x) \varphi^{F}(a) & \left(x^{+}>a^{+}, x^{-}<a^{-}\right) \\
\psi(x) \varphi^{F}(a) & \left(x^{+}<a^{+}, x^{-}>a^{-}\right) .\end{cases}
\end{aligned}
$$

The commutation relations among $\varphi_{F}^{\prime}$ 's and $\varphi^{F}$ 's read

$$
\begin{array}{ll}
\varphi_{F}(a) \varphi_{F}\left(a^{\prime}\right)=\varphi_{F}\left(a^{\prime}\right) \varphi_{F}(a) & \left(\left(a^{+}-a^{++}\right)\left(a^{-}-a^{\prime-}\right)<0\right), \\
\varphi^{F}(a) \varphi^{F}\left(a^{\prime}\right)=\varphi^{F}\left(a^{\prime}\right) \varphi^{F}(a) & \left(\left(a^{+}-a^{++}\right)\left(a^{-}-a^{\prime-}\right)<0\right),
\end{array}
$$

(4.6. 8) $\quad \varphi_{F}(a) \varphi^{F}\left(a^{\prime}\right)= \begin{cases}\varphi^{F}\left(a^{\prime}\right) \varphi_{F}(a) & \left(a^{\prime+}>a^{+}, a^{\prime-}<a^{-}\right) \\ -\varphi^{F}\left(a^{\prime}\right) \varphi_{F}(a) & \left(a^{\prime+}<a^{+}, a^{\prime-}>a^{-}\right) .\end{cases}$

The relations (4.6.4), (4.6.5), (4.6.7) and (4.6.8) are proved by a similar method employed in the previous section. Essentially they are reduced to the local commutativity of $\varphi_{B}(a)$ 's (resp. $\varphi_{F}(a)$ 's) and the local expansion (4.3.26)' (resp. (4.3.67)). It is also possible to give a direct, computational proof to the former. Since the arguments are almost the same, we consider here the case of $\varphi_{B}(a)$.

The norm of the product $\varphi_{B}\left(a_{1}\right) \varphi_{B}\left(a_{2}\right)$ is obtained by applying the product formula (A. 25). We have

$$
\begin{aligned}
& \operatorname{Nr}\left(\varphi_{B}\left(a_{1}\right) \varphi_{B}\left(a_{2}\right)\right)=\left\langle\varphi_{B}\left(a_{1}\right) \varphi_{B}\left(a_{2}\right)\right\rangle e^{\rho\left(a_{1}, a_{2}\right) / 2} \\
& \rho\left(a_{1}, a_{2}\right)=\iint d u \underline{d u^{\prime}} R\left(u, u^{\prime} ; a_{1}, a_{2}\right) \phi(u) \phi\left(u^{\prime}\right)
\end{aligned}
$$

where the kernel $R\left(u, u^{\prime} ; a_{1}, a_{2}\right)$ is given by the following infinite series:

(4. 6. 10) $R\left(u, u^{\prime} ; a_{1}, a_{2}\right)=\sum_{\mu, \nu=1,2} \sum_{l=0}^{\infty} R_{\mu \nu}^{(l)}\left(u, u^{\prime} ; a_{1}, a_{2}\right)$,

(4. 6. 11) $)_{11} \quad R_{11}^{(l)}\left(u, u^{\prime} ; a_{1}, a_{2}\right)$

$$
\begin{aligned}
& =\int_{0}^{\infty} \cdots \int_{0}^{\infty} d u_{1} \cdots d u_{2 l} \frac{-2 \sqrt{u-i 0} \sqrt{u_{1}}-2 \sqrt{u_{1}} \sqrt{u_{2}}}{u+u_{1}-i 0} \frac{-2 \sqrt{u_{2 l}} \sqrt{u^{\prime}-i 0}}{u_{1}+u_{2}} \\
& \times \exp \left\{-i m\left(\left(a_{1}^{-}-a_{2}^{-}\right) U_{2 l}+\left(a_{1}^{+}-a_{2}^{+}\right) U_{2 l}^{\prime}\right)\right. \\
& \left.-i m\left(a_{1}^{-}\left(u+u^{\prime}\right)+a_{1}^{+}\left(u^{-1}+u^{\prime-1}\right)\right)\right\},
\end{aligned}
$$

$(4.6 .11)_{12} \quad R_{12}^{(l)}\left(u, u^{\prime} ; a_{1}, a_{2}\right)$

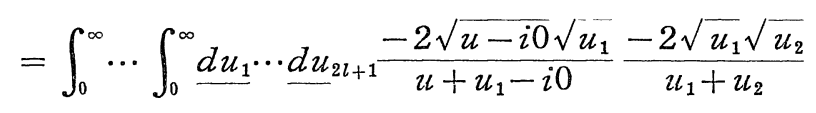




$$
\begin{aligned}
& \ldots \frac{-2 \sqrt{-u_{2 l+1}-i 0} \sqrt{u^{\prime}-i 0}}{-u_{2 l+1}+u^{\prime}-i 0} \times \exp \left\{-i m\left(\left(a_{1}^{-}-a_{2}^{-}\right) U_{2 l+1}\right.\right. \\
& \left.\left.+\left(a_{1}^{+}-a_{2}^{+}\right) U_{2 l+1}^{\prime}\right)-i m\left(a_{1}^{-} u+a_{1}^{+} u^{-1}+a_{2}^{-} u^{\prime}+a_{2}^{+} u^{\prime-1}\right)\right\},
\end{aligned}
$$

$(4.6 .11)_{21} \quad R_{21}^{(l)}\left(u, u^{\prime} ; a_{1}, a_{2}\right)$

$$
\begin{aligned}
= & \int_{0}^{\infty} \cdots \int_{0}^{\infty} \frac{d u_{1}}{\cdots} \frac{d u_{2 l+1}}{-2 \sqrt{u-i 0} \sqrt{-u_{1}-i 0}}-2 \sqrt{u_{1}} \sqrt{u_{2}} \\
u-u_{1}-i 0 & u_{1}+u_{2} \\
& \ldots \frac{-2 \sqrt{u_{2 l+1}} \sqrt{u^{\prime}-i 0}}{u_{2 l+1}+u^{\prime}-i 0} \times \exp \left\{-i m\left(\left(a_{1}^{-}-a_{2}^{-}\right) U_{2 l+1}\right.\right. \\
& \left.\left.+\left(a_{1}^{+}-a_{2}^{+}\right) U_{2 l+1}^{\prime}\right)-i m\left(a_{2}^{-} u+a_{2}^{+} u^{-1}+a_{1}^{-} u^{\prime}+a_{1}^{+} u^{\prime-1}\right)\right\},
\end{aligned}
$$

(4. 6. 11) ${ }_{22} \quad R_{22}^{(l)}\left(u, u^{\prime} ; a_{1}, a_{2}\right)$

$$
\begin{aligned}
= & \int_{0}^{\infty} \cdots \int_{0}^{\infty} \frac{d u_{1}}{\cdots} \frac{d u_{2 l}}{\frac{-2 \sqrt{u-i 0}}{u-u_{1}-i 0}} \frac{-2 \sqrt{u_{1}} \sqrt{u_{2}}}{u-u_{1}-i 0} \\
& \ldots \frac{-2 \sqrt{-u_{2 l}-i 0} \sqrt{u^{\prime}-i 0}}{-u_{2 l}+u^{\prime}-i 0} \times \exp \left\{-i m\left(\left(a_{1}^{-}-a_{2}^{-}\right) U_{2 l}+\left(a_{1}^{+}-a_{2}^{+}\right) U_{2 l}^{\prime}\right)\right. \\
& \left.-i m\left(a_{2}^{-}\left(u+u^{\prime}\right)+a_{2}^{+}\left(u^{-1}+u^{\prime-1}\right)\right)\right\}
\end{aligned}
$$

where $U_{k}=u_{1}+\cdots+u_{k}, U_{k}^{\prime}=u_{1}^{-1}+\cdots+u_{k}^{-1} . \quad$ A change of countours ${ }^{(*)} u_{j} \mapsto$ $e^{i \theta} u_{j}$ shows that (4.6.10) is convergent for real $a_{1}, a_{2} \in X^{\text {Min }}$ provided $-\left(a_{1}^{-}-a_{2}^{-}\right)\left(a_{1}^{+}-a_{2}^{+}\right)$is sufficiently large. We emphasize here that it is analytically prolongable to the whole region where $a_{1}-a_{2}$ is spacelike. For, as shown in the previous section (cf. Proof of Theorem 4.5.6) the wave functions corresponding to the kernel (4.6.10) gives the canonical basis $v_{0}\left(z^{*}, z\right)$ of $W_{B, z^{*}, a_{1}, a_{2}}^{0, \frac{1}{2}, \frac{1}{2}}$ at least if $a_{1}, a_{2} \in X^{\mathrm{Min}} \cap X^{\mathrm{Euc}}$ and $\left|a_{1}-a_{2}\right| \gg 1$. On the other hand, existence and analyticity of the latter has already been proved in Chapter III for arbitrary distinct $a_{1}, a_{2} \in X^{\text {Euc }}$.

From the Lorentz covariance it readily follows that $\left\langle\varphi_{B}\left(a_{1}\right) \varphi_{B}\left(a_{2}\right)\right\rangle$ $=\left\langle\varphi_{B}\left(a_{2}\right) \varphi_{B}\left(a_{1}\right)\right\rangle$. One may check this directly by a similar infinite series expression (cf. (4.6.67)). Hence our problem is to prove the following:

Proposition 4. 6. 1. If $\left(a_{1}^{-}-a_{2}^{-}\right)\left(a_{1}^{+}-a_{2}^{+}\right)<0$, we have

$$
R\left(u, u^{\prime} ; a_{1}, a_{2}\right)=R\left(u, u^{\prime} ; a_{2}, a_{1}\right) .
$$

(*) Contours for $u_{1}, u_{22}$ or $u_{2 l+1}$ in $(4.6 .11)_{\mu \nu}$ should be properly modified in accordance with the rule $u+u_{1}-i 0$, etc. 
Proof. By the remark given above, it suffices to prove (4.6.12) assuming $\left|a_{1}-a_{2}\right| \gg 1$. Set $R_{\mu \nu}^{(l)}=R_{\mu \nu}^{(l)}\left(u, u^{\prime} ; a_{1}, a_{2}\right), R_{\mu \nu}^{\prime(l)}=R_{\mu \nu}^{(l)}\left(u, u^{\prime} ; a_{2}, a_{1}\right)$. Without loss of generality we assume $a_{1}^{-}-a_{2}^{-}<0, a_{1}^{+}-a_{2}^{+}>0$ and show that

$$
R_{11}^{(l)}-R_{22}^{(l)}=-2 R_{21}^{(l-1)}+2 R_{21}^{\prime(l-1)},
$$

$$
R_{12}^{(l)}+R_{21}^{\prime(l)}=-2 R_{22}^{(l)} \text {, }
$$

$$
R_{21}^{(l)}+R_{12}^{\prime(l)}=-2 R_{11}^{(l)},
$$

$$
R_{22}^{(l)}-R_{11}^{\prime(l)}=0 \text {. }
$$

This is sufficient for our purpose, since then we shall have

$$
\begin{aligned}
R & \left(u, u^{\prime} ; a_{1}, a_{2}\right)-R\left(u, u^{\prime} ; a_{2}, a_{1}\right) \\
& =\sum_{l=0}^{\infty}\left\{R_{11}^{(l)}-R_{22}^{\prime(l)}+R_{12}^{(l)}-R_{21}^{\prime(l)}+R_{21}^{(l)}-R_{12}^{\prime(l)}+R_{22}^{(l)}-R_{11}^{\prime(l)}\right\} \\
& =\sum_{l=0}^{\infty}\left(-2 R_{21}^{(l)}+2 R_{21}^{\prime(l)}+R_{12}^{(l)}-R_{21}^{\prime(l)}+R_{21}^{(l)}-R_{12}^{(l)}\right) \\
& =\sum_{l=0}^{\infty}\left(-2 R_{22}^{(l)}-\left(-2 R_{11}^{\prime(l)}\right)\right) \\
& =0
\end{aligned}
$$

as desired.

To prove (4.6.13) ${ }_{l}$ we rewrite the left hand side in the form

$(4.6 .17)_{l} \quad R_{11}^{(l)}-R_{22}^{\prime(l)}$

$$
\begin{gathered}
=e^{-i m\left(a_{1}^{-} u+a_{1}^{+} u^{-1}+a_{2}^{-} u^{\prime}+a_{2}^{+} u^{\prime-1}\right)} \sum_{k=1}^{2 l} I_{k}^{(l)} \\
I_{k}^{(l)}=\int_{-\infty}^{0} \cdots \int_{-\infty}^{0} \frac{d u_{1} \cdots d u_{k-1} \int_{-\infty}^{+\infty} \frac{d u_{k}}{2 \pi u_{k}} \int_{0}^{\infty} \cdots \int_{0}^{\infty} d u_{k+1} \cdots d u_{2 l}}{} \\
\quad \times \prod_{j=1}^{2 l+1} \frac{-2 \sqrt{u_{j-1}-i 0} \sqrt{u_{j}-i 0}}{u_{j-1}+u_{j}-i 0} \\
\times e^{-i m\left(\left(a_{1}^{-}-a_{2}^{-}\right) u_{j}+\left(a_{1}^{+}-a_{2}^{+}\right) u_{j}^{-1}\right)}
\end{gathered}
$$

where $u_{0}=u$ and $u_{2 l+1}=u^{\prime}$. By assumption $a_{1}^{-}-a_{2}^{-}<0$ and $a_{1}^{+}-a_{2}^{+}>0$, hence we may close the contour of $u_{k}$-integration in the upper half $u_{k}$ plane. Evaluating the residues we obtain $I_{k}^{(l)}=J_{k-1}^{(l)}-J_{k}^{(l)}$ with

$(4.6 .18)_{l k} \quad J_{k}^{(l)}=2 i \int_{-\infty}^{0} \cdots \int_{-\infty}^{0} \prod_{j=1}^{k-1} d u_{j} \int_{0}^{\infty} \cdots \int_{0}^{\infty} \prod_{j=k+1}^{2 l} d u_{j}$ 


$$
\begin{aligned}
& \times \frac{-2 \sqrt{u_{k-1}-i 0} \sqrt{u_{k+1}}}{u_{k-1}-u_{l_{k+1}}} \prod_{\substack{j=1 \\
j \neq k, k+1}}^{2 l+1} \frac{-2 \sqrt{u_{j-1}-i 0} \sqrt{u_{j}-i 0}}{u_{j-1}+u_{j}-i 0} \\
& \times e^{-i m\left(\left(a_{1}^{-}-a_{2}^{-}\right) u_{j}+\left(a_{1}^{+}-a_{2}^{+}\right) u_{j}^{-1}\right)} \quad(1 \leqq k \leqq 2 l) \\
& =2 i \int_{0}^{\infty} \cdots \int_{0}^{\infty} \prod_{j=2}^{2 l} \frac{d u_{j} \cdot \frac{-2 \sqrt{u_{0}-i 0} \sqrt{u_{0}}}{u_{0}-u_{2}}}{\times e^{-i m\left(\left(a_{1}^{-}-a_{2}^{-}\right)\left(-u_{0}+u_{2}\right)+\left(a_{1}^{+}-a_{2}^{+}\right)\left(-u_{0}^{-1}+u_{2}^{-1}\right)\right)}} \\
& \times \prod_{j=3}^{2 l+1} \frac{-2 \sqrt{u_{j-1}-i 0} \sqrt{u_{j}-i 0}}{u_{j-1}+u_{j}-i 0} e^{-i m\left(\left(a_{1}^{-}-a_{2}^{-}\right) u_{j}+\left(a_{1}^{+}-a_{2}^{+}\right) a_{j}^{-1}\right)} \\
& \quad(k=0) .
\end{aligned}
$$

From (4.6.17), and (4.6.18) $)_{l k}$ we have

$$
\begin{aligned}
R_{11}^{(l)}-R_{22}^{\prime(l)} & =e^{-i m\left(a_{1}^{-} u+a_{1}^{+} u^{-1}+a_{2}^{-} u^{\prime}+a_{2}^{+} u^{\prime-1}\right)}\left(J_{0}^{(l)}-J_{2 l}^{(l)}\right) \\
& =-2 R_{21}^{(l-1)}+2 R_{21}^{\prime(l-1)} .
\end{aligned}
$$

We see also that if $a_{1}^{-}-a_{2}^{-}>0$ and $a_{1}^{+}-a_{2}^{+}<0$, then all the $I_{k}^{(l)}$ 's yield 0. Exchanging $a_{1}$ and $a_{2}$ we have (4.6.16) ${ }_{l}$. The identities (4.6.14) and (4.6.15) $)_{\imath}$ are proved by a similar calculation. We leave the details to the reader.

The fields $\varphi^{B}(a)$ and $\varphi^{F}(a)$ are of particular interest because they possess asymptotic fields. We set

$$
\begin{aligned}
& \text { (4. 6. 19) } \phi_{\varepsilon \pm}^{B}(u)=\lim _{t \rightarrow \pm \infty} \frac{i \epsilon(u)}{2} \int_{x^{0}=t} d x^{1}\left(e^{i m\left(x-u+x^{+} u^{-1}\right)} \partial_{0} \varphi_{\varepsilon}^{B}(x)\right. \\
& \left.-\varphi_{\varepsilon}^{B}(x) \partial_{0} e^{i m\left(x-u+x^{+} u-1\right)}\right), \\
& (\varepsilon=+ \text { or }-)
\end{aligned}
$$$$
\text { (4.6.20) } \phi_{ \pm}^{F}(u)=\lim _{t \rightarrow \pm \infty} \frac{i \epsilon(u)}{2} \int_{x^{0}=t} d x^{1}\left(e^{i m\left(x^{-} u+x^{r} u^{-1}\right)} \partial_{0} \varphi^{F}(x)\right.
$$$$
\left.-\varphi^{F}(x) \partial_{0} e^{i m\left(x^{-} u+x^{+} u^{-1}\right)}\right) .
$$

Here the limit is taken in the weak sense. Namely in (4.6.19) a limit of the form $\lim _{t \rightarrow \pm \infty} \sum_{k=0}^{\infty} \frac{1}{k !} \int \cdots \int \underline{d u_{1}} \cdots d u_{k} \rho_{k}\left(u_{1}, \cdots, u_{k} ; t\right): \phi\left(u u_{k}\right) \cdots \phi\left(u_{1}\right):$ is 
understood to be $\sum_{k=0}^{\infty} \frac{1}{k !} \int \cdots \int \underline{d u_{1}} \cdots \underline{d u_{k}}\left(\lim _{t \rightarrow \pm \infty} \rho_{k}\left(u_{1}, \cdots, u_{k} ; t\right)\right): \phi\left(u_{k}\right) \cdots$ $\phi\left(u_{1}\right)$ :, and similarly for (4.6.20).

Proposition 4. 6.2. We have

$$
\phi_{\varepsilon \pm}^{B}(u)=(0+i u)^{\varepsilon \frac{1}{2}} \psi_{ \pm}^{B}(u) \quad(\varepsilon= \pm)
$$

where

(4. 6. 22) $\operatorname{Nr}\left(\psi_{ \pm}^{B}(u)\right)=\phi(u) \cdot \exp \left(-2 \int_{0}^{\infty} d u^{\prime} \theta\left( \pm\left(|u|-u^{\prime}\right) \phi^{\prime}\left(u^{\prime}\right) \phi\left(u^{\prime}\right)\right)\right.$.

Likervise we have

(4. 6. 23) $\quad \operatorname{Nr}\left(\phi_{ \pm}^{F}(u)\right)=\psi(u)$

$$
\times \exp \left(-2 \int_{0}^{\infty} d u^{\prime} \theta\left( \pm\left(|u|-u^{\prime}\right)\right) \psi^{\dagger}\left(u^{\prime}\right) \psi\left(u^{\prime}\right)\right) .
$$

Here we have set $\phi^{\dagger}(u)=\phi(-u), \psi^{\dagger}(u)=\psi(-u)$.

Proof. We shall prove (4.6.21) and (4.6.22). Calculation of (4.6.23) is quite similar.

By the definition we have

$$
\begin{aligned}
& \text { (4. 6. 24) } \operatorname{Nr}\left(\phi_{\varepsilon \pm}^{B}(u)\right)=\sum_{k=0}^{\infty} \frac{1}{k !} \phi_{\varepsilon \pm}^{B(k)}(u) \\
& \phi_{\varepsilon \pm}^{B(k)}(u)=\lim _{t \rightarrow \pm \infty} \frac{i \epsilon(u)}{2} \int_{x^{0}=t} d x^{1}\left\{\left(\rho_{B}(x) / 2\right)^{k}\right. \\
& \times\left(e^{i m\left(x^{-} u+x^{+} u^{-1}\right)} \partial_{0} \phi_{\varepsilon}(x)-\phi_{\varepsilon}(x) \partial_{0} e^{i m\left(x-u+x^{+} u-1\right)}\right) \\
& \left.+k\left(\rho_{B}(x) / 2\right)^{k-1} \phi_{\varepsilon}(x) \cdot e^{i m\left(x-u+x^{+} u-1\right)} \partial_{0} \rho_{B}(x) / 2\right\} \\
& =\lim _{t \rightarrow \pm \infty} \int \cdots \int d u_{0} \cdots d u_{2 k} \rho_{2 k+1}\left(u ; u_{0}, \cdots, u_{2 k} ; t\right) \phi\left(u_{2 k}\right) \cdots \phi\left(u_{0}\right) \text {. }
\end{aligned}
$$

Here the coefficient $\rho_{2 k+1}\left(u ; u_{0}, \cdots, u_{2 k} ; t\right)$, after $x_{1}$-integration, is given by (4.6.25) $\rho_{2 k+1}\left(u ; u_{0}, \cdots, u_{2 k} ; t\right)$

$$
\begin{aligned}
= & \left(0+i u_{0}\right)^{\varepsilon \frac{1}{2}} \frac{\epsilon(u)}{2}\left(\left(u+u_{0}\right)\left(1+\frac{1}{u u_{0}}\right)+\sum_{j=1}^{k}\left(u_{2 j-1}+u_{2 j}\right)\right. \\
& \left.\times\left(1+\frac{1}{u_{2 j-1} u_{2 j}}\right)\right) \prod_{j=1}^{k} \frac{-\sqrt{u_{2 j-1}-i 0} \sqrt{u_{2 j}-i 0}}{u_{2 j-1}+u_{2 j}-i 0}
\end{aligned}
$$




$$
\begin{aligned}
& \times \exp \left(-\frac{i m t}{2}\left(u_{2 j-1}+u_{2 j}\right)\left(1+\frac{1}{u_{2 j-1} u_{2 j}}\right)\right) \\
& \times \exp \left(\frac{i m t}{2}\left(u-u_{0}\right)\left(1-\frac{1}{u u_{0}}\right)\right) \cdot 2 \pi \delta\left(\left(u-u_{0}\right)\left(1+\frac{1}{u u_{0}}\right)\right. \\
& \left.-\sum_{j=1}^{k}\left(u_{2 j-1}+u_{2 j}\right)\left(1-\frac{1}{u_{2 j-1} u_{2 j}}\right)\right) .
\end{aligned}
$$

At $1+\frac{1}{u u_{0}}=0$, the coefficients of the exponential factors are real analytic (except for the delta function), so that $1+\frac{1}{u u_{0}}=0$ has no contribution in the limit $t \rightarrow \pm \infty$ by the Riemann-Lebesgue lemma. Outside there the exponential factors are written as $\prod_{j=1}^{k} \exp \left(-i m t\left(u_{2 j-1}+u_{2 j}\right) \cdot\left(\frac{1}{u u_{0}}+\frac{1}{u_{2 j-1} u_{2 j}}\right)\right.$ $\left.\times\left(1+\frac{1}{u u_{0}}\right)^{-1}\right)$. Applying the formula

$$
\lim _{t \rightarrow \pm \infty} \frac{-i}{x-i 0} e^{-i x a t}=\theta(\mp a) 2 \pi \delta(x)
$$

we then obtain

$$
\text { (4.6. 26) } \begin{aligned}
& \lim _{t \rightarrow \pm \infty} \rho_{2 k+1}\left(u ; u_{0}, \cdots, u_{2 k} ; t\right) \\
&=(0+i u)^{\varepsilon \frac{1}{2}} 2 \pi|u| \delta\left(u-u_{0}\right) \\
& \times \prod_{j=1}^{k}\left(-\theta\left( \pm\left(u^{2}-u_{2 j-1}^{2}\right)\right)\right) 2 \pi\left|u_{2 j-1}\right| \delta\left(u_{2 j-1}+u_{2 j}\right) .
\end{aligned}
$$

This shows that

$$
\text { (4. 6. 27) } \begin{aligned}
\phi_{\varepsilon \pm}^{F(k)}(u) & =(0+i u)^{\varepsilon^{\frac{1}{2}}} \psi_{ \pm}^{B(k)}(u), \\
\phi_{ \pm}^{B(k)}(u) & =\phi(u)\left(-2 \int_{0}^{\infty} d u^{\prime} \theta\left( \pm\left(|u|-u^{\prime}\right)\right) \phi^{\dagger}\left(u^{\prime}\right) \phi\left(u^{\prime}\right)\right)^{k}
\end{aligned}
$$

and we have (4.6.22). This completes the proof of Proposition 4.6.2.

For $u>0$ we set

$$
\begin{aligned}
& \text { (4. 6. 28) } \\
& \mathrm{N}_{B}^{ \pm}(u)=\int_{0}^{\infty} \frac{d u^{\prime} \theta\left( \pm\left(u-u^{\prime}\right)\right) \phi^{\dagger}\left(u^{\prime}\right) \phi\left(u^{\prime}\right)}{\text { (4. 6. 29) }} \mathrm{N}_{F}^{ \pm}(u)=\int_{0}^{\infty} \underline{d u^{\prime}} \theta\left( \pm\left(u-u^{\prime}\right)\right) \phi^{\dagger}\left(u^{\prime}\right) \phi\left(u^{\prime}\right) .
\end{aligned}
$$

Then (4.6.22) and (4.6.23) are respectively written as (cf. (2.1.32)- 
(2.1.33))

$$
\begin{aligned}
& \phi_{ \pm}^{B \dagger}(u)=\phi^{\dagger}(u) \cdot(-)^{\mathrm{N}_{B}^{ \pm}(u)}, \\
& \phi_{ \pm}^{B}(u)=(-)^{\mathrm{N}_{B}^{ \pm}(u)} \cdot \phi(u), \\
& \phi_{ \pm}^{F^{\dagger}}(u)=\phi^{\dagger}(u) \cdot(-)^{\mathrm{N}_{F}^{ \pm}(u)}, \\
& \phi_{ \pm}^{F}(u)=(-)^{\mathrm{N}_{F}^{ \pm}(u)} \cdot \phi(u),
\end{aligned}
$$

where $u>0$ and $\psi_{ \pm}^{B \dagger}(u)=\psi_{ \pm}^{B}(-u), \quad \phi_{ \pm}^{F \dagger}(u)=\phi_{ \pm}^{F}(-u)$. In particular $\psi_{ \pm}^{B \dagger}(u) \psi_{ \pm}^{B}(u)=\phi^{\dagger}(u) \phi(u), \phi_{ \pm}^{F \dagger}(u) \phi_{ \pm}^{F}(u)=\phi^{\dagger}(u) \psi(u) \quad$ hold. Regarding (4.6.28)-(4.6.29) we see that the correspondences $\phi(u) \mapsto \psi_{\varepsilon}^{B}(u)$ and $\psi(u) \mapsto \phi_{ \pm}^{F^{F}}(u) \quad(\varepsilon=+$ or -$)$ are mutually inverse operations. Making use of (4.6.30)-(4.6.31) we may verify the following canonical anti-commutation (resp. commutation) relations for $\psi_{ \pm}^{B}(u)$ (resp. $\phi_{ \pm}^{F}(u)$ ):

$$
\begin{aligned}
& {\left[\psi_{\varepsilon}^{B}(u), \psi_{\varepsilon}^{B}\left(u^{\prime}\right)\right]_{+}=2 \pi|u| \delta\left(u+u \iota^{\prime}\right),} \\
& {\left[\phi_{\varepsilon}^{F}(u), \phi_{\varepsilon}^{F}\left(u^{\prime}\right)\right]=2 \pi u \delta\left(u+u^{\prime}\right),}
\end{aligned}
$$

where $\varepsilon=+$ or - . The relations between the asymptotic and auxiliary state vectors are also derived from (4.6.30)-(4.6.31).

Proposition 4.6.3. We have

$$
\begin{aligned}
& \langle\operatorname{vac}| \psi_{ \pm}^{B}\left(u_{k}\right) \cdots \psi_{ \pm}^{B}\left(u_{1}\right)=\prod_{i \gtrless j} \epsilon\left(u_{i}-u_{j}\right) \cdot\langle\operatorname{vac}| \phi\left(u_{k}\right) \cdots \phi\left(u_{1}\right) \\
& \psi_{ \pm}^{B \dagger}\left(u_{1}\right) \cdots \psi_{ \pm}^{B \dagger}\left(u_{k}\right)|v a c\rangle=\prod_{i \gtrless_{j}} \epsilon\left(u u_{i}-u_{j}\right) \cdot \phi^{\dagger}\left(u_{1}\right) \cdots \phi^{\dagger}\left(u u_{k}\right)|v a c\rangle .
\end{aligned}
$$

The same relations hold if we replace $\psi_{ \pm}^{B}(u), \phi(u)$ by $\phi_{ \pm}^{F}(u), \psi(u)$ respectively.

Corollary 4. 6. 4. The S-matrix for $\varphi^{B}(a)$ (resp. $\left.\varphi^{F}(a)\right)$ is given by

$$
S=(-)^{\mathrm{N}(\mathrm{N}-1) / 2}
$$

where $\mathrm{N}=\mathrm{N}_{B}^{+}(\infty)$ (resp. $\mathrm{N}_{F}^{+}(\infty)$ ) denotes the total particle-number operator.

We omit the proof.

Summing up we have shown the following. On the one hand the 
field $\varphi^{B}(a)$, constructed from free boson, is an interacting fermion field satisfying the Lorentz covariance, microcausality and asymptotic completeness condition. The field $\varphi^{F}(a)$, on the other hand, is constructed from free fermion and behaves as an interacting boson with the above mentioned properties. Moreover their asymptotic fields are in a reciprocal relation, and their $S$-matrices coincide.

Remark. It is instructive to note the reciprocity at the level of the correspondence between the rotation $T$ and the kernel $R$. In general let $W_{F}$ be an orthogonal vector space, and let \langle\rangle denote the expectation value corresponding to a holonomic decomposition $W_{F}=V^{*} \oplus V$. Setting $\left\langle w, w^{\prime}\right\rangle_{B}=\left\langle w w^{\prime}\right\rangle-\left\langle w^{\prime} w\right\rangle$ we see that the underlying vector space of $W_{F}$ is also endowed with a structure of a symplectic space, which we denote by $W_{B}$. As usual $K, J=K+{ }^{t} K$ and $H=K-{ }^{t} K$ will denote the tables of expectation values, the orthogonal and symplectic inner product respectively. Let now $T=T_{F}$ be a rotation in $W_{F}$, and let $S$ be its conjugate transform (Section 1.2). Then $S=T_{B}$ is a symplectic rotation i.e. ${ }^{t} T_{B} H T_{B}=H$ if and only if $T^{2}=1$ (or equivalently $S^{2}=1$ by Proposition 1. 2. 2). In this case the matrices $R_{F}=\left(T_{F}-1\right)\left(K+{ }^{t} K T_{F}\right)^{-1}$ and $R_{B}$ $=\left(T_{B}-1\right)\left(K-{ }^{t} K T_{B}\right)^{-1}$ are reciprocally related through

$$
R_{F} H=T_{B}-1, R_{B} J=T_{F}-1 .
$$

As remarked in p. 910, the symplectic space $W_{B}$ in Section 4.1 and the orthogonal $W_{F}$ in Section 4.2 are the same objects, on which the symplectic and orthogonal rotations $T_{B}=T_{\varphi_{B}(0)}=1-2 P_{B}, \quad T_{F}=T_{\varphi_{F}(0)}=1$ $-2 P_{F}$ are defined to satisfy $T_{B}^{2}=1, T_{F}^{2}=1$. Indeed these are mutually conjugate, and it is easy to verify directly that

$$
\begin{aligned}
& P_{B}\left(u, u^{\prime}\right)=\frac{1}{2} \frac{-i\left(u+u^{\prime}\right)}{u-u^{\prime}-i 0} \epsilon\left(u^{\prime}\right)=\frac{1}{2} R_{F}\left(u,-u^{\prime}\right) \epsilon\left(u^{\prime}\right) \\
& P_{F}\left(u, u^{\prime}\right)=\frac{-i \sqrt{u-i 0} \sqrt{u^{\prime}+i 0}}{u-u^{\prime}-i 0}=-\frac{1}{2} R_{B}\left(u,-u^{\prime}\right)
\end{aligned}
$$

The $\tau$ functions for these fields are obtained exactly by relating them to the Euclidean deformation theory, just as in the complex case discussed in Section 4. 4. Let us now examine the properties of wave functions. We set 
(4.6.36)

$$
\begin{aligned}
& w_{B}(y, x)=\left\langle\phi(y) \varphi_{B}\left(a_{1}\right) \cdots \varphi_{B}\left(a_{n}\right) \phi(x)\right\rangle, \\
& w_{B \nu \pm}(x)=\left\langle\varphi_{B}\left(a_{1}\right) \cdots \varphi_{ \pm}^{B}\left(a_{\nu}\right) \cdots \varphi_{B}\left(a_{n}\right) \phi(x)\right\rangle, \\
& w_{F}(y, x)_{\varepsilon \varepsilon^{\prime}}=\left\langle\psi_{\varepsilon^{\prime}}(y) \varphi_{F}\left(a_{1}\right) \cdots \varphi_{F}\left(a_{n}\right) \psi_{\varepsilon}(x) \quad\left(\varepsilon, \varepsilon^{\prime}= \pm\right),\right. \\
& w_{F_{\nu}}(x)_{=}=\left\langle\varphi_{F}\left(a_{1}\right) \cdots \varphi^{F}\left(a_{\nu}\right) \cdots \varphi_{F}\left(a_{n}\right) \psi_{=}(x)\right\rangle,
\end{aligned}
$$

and

$$
\begin{aligned}
& \tau_{D n}=\tau_{B n}\left(a_{1}, \cdots, a_{n}\right)=\left\langle\varphi_{B}\left(a_{1}\right) \cdots \varphi_{B}\left(a_{n}\right)\right\rangle, \\
& \tau_{F n}=\tau_{F n}\left(a_{1}, \cdots, a_{n}\right)=\left\langle\varphi_{F}\left(a_{1}\right) \cdots \varphi_{F}\left(a_{n}\right)\right\rangle .
\end{aligned}
$$

Here also we assume $y^{{ }^{\top}}>a_{1}^{+}>\cdots>a_{n}^{+}$for the sake of definiteness. Local expansions for (4.6.36) and (4.6.37) are obtained by applying (4.3. $46)-(4.3 .47)$, (4.3.67)-(4.3.68) respectively. As remarked there, formulas for $\mathfrak{w}_{B}$ 's take the same form as in the complex case, whereas those for $w_{F}$ 's are slightly changed. The results are as follows.

$(4.6 .39)_{0} \quad \varkappa_{B}(y, x)=\frac{\tau_{B n}}{\pi} \widetilde{v}_{0}[y]$

$$
+\sum_{j=-\infty}^{+r o}\left\langle: \phi(y) \phi_{j}(y): \varphi_{B}\left(a_{1}\right) \cdots \varphi_{B}\left(a_{n}\right)\right\rangle v_{j}[y] .
$$

(4.6.39),

$$
\begin{aligned}
& \mathcal{w}_{B}(y, x)=\sum_{j=0}^{\infty}\left\langle\phi(y) \varphi_{B}\left(a_{1}\right) \cdots \varphi_{\frac{1}{2}+j}^{B}\left(a_{\nu}\right) \cdots \varphi_{B}\left(a_{n}\right)\right\rangle v_{\frac{1}{2}+j}\left[a_{\nu}\right] \\
& +\sum_{j=0}^{\infty}\left\langle\phi(y) \varphi_{B}\left(a_{1}\right) \cdots \varphi_{-\frac{1}{2}-j}^{B}\left(a_{\nu}\right) \cdots \varphi_{B}\left(a_{n}\right)\right\rangle v_{\frac{1}{2}+j}^{*}\left[a_{\nu}\right] .
\end{aligned}
$$

$(4.6 .40)_{\mu \nu}$

$$
\begin{aligned}
& w_{B \mu \pm}(x)=\sum_{j=0}^{\infty}\left\langle\varphi_{B}\left(a_{1}\right) \cdots \varphi_{ \pm \frac{1}{2}}^{B}\left(a_{\mu}\right) \cdots \varphi_{\frac{1}{2}+j}^{B}\left(a_{\nu}\right) \cdots \varphi_{B}\left(a_{n}\right)\right\rangle v_{\frac{1}{2}+j}^{*}\left[a_{\nu}\right] \\
& +\sum_{j=0}^{\infty}\left\langle\varphi_{B}\left(a_{1}\right) \cdots \varphi_{ \pm \frac{1}{2}}^{B}\left(a_{\mu}\right) \cdots \varphi_{-\frac{1}{2}-j}^{B}\left(a_{\nu}\right) \cdots \varphi_{B}\left(a_{n}\right)\right\rangle v_{\frac{1}{2}+j}^{*}\left[a_{\nu}\right], \\
& w_{B \mu+}(x)=\frac{1}{2} \tau_{B n} v_{-\frac{1}{2}}\left[a_{\mu}\right]+\sum_{j=0}^{c}\left\langle\varphi_{B}\left(a_{1}\right) \cdots \varphi_{\frac{1}{2}, \frac{1}{2}+j}^{B}\left(a_{\mu}\right) \cdots \varphi_{B}\left(a_{n}\right)\right\rangle v_{\frac{1}{2}+j}\left[a_{\mu}\right] \\
& +\sum_{j=0}^{\infty}\left(-\frac{1}{2} \delta_{j 0} \tau_{B}+\left\langle\varphi_{B}\left(a_{1}\right) \cdots \varphi_{\frac{1}{2},-\frac{1}{2}-j}^{B}\left(a_{\mu}\right) \cdots \varphi_{B}\left(a_{n}\right)\right\rangle\right) v_{\frac{i}{2}+j}^{*}\left[a_{\mu}\right], \\
& w_{B \mu-}(x)=\sum_{j=0}^{\infty}\left(-\frac{1}{2} \delta_{j 0} \tau_{B}+\left\langle\varphi_{B}\left(a_{1}\right) \cdots \varphi_{-\frac{1}{2}, \frac{1}{2}+j}^{B}\left(a_{\mu}\right) \cdots \varphi_{B}\left(a_{n}\right)\right\rangle\right) v_{\frac{1}{2}+j}\left[a_{\mu}\right]
\end{aligned}
$$




$$
\begin{gathered}
+\frac{1}{2} \tau_{B n} v_{-\frac{1}{2}}^{*}\left[a_{\mu}\right]+\sum_{j=0}^{\infty}\left\langle\varphi_{B}\left(a_{1}\right) \cdots \varphi_{-\frac{1}{2},-\frac{1}{2}-j}^{B}\left(a_{\mu}\right) \cdots \varphi_{B}\left(a_{n}\right)\right\rangle v_{\frac{1}{2}+j}^{*}\left[a_{\mu}\right] \\
\text { (if } \mu=\nu) .
\end{gathered}
$$

We note that (4.3.45) implies

$$
\text { (4.6.41) } \begin{aligned}
& \left\langle\varphi_{B}\left(a_{1}\right) \cdots \varphi_{\frac{1}{2}, \frac{1}{2}}^{B}\left(a_{\mu}\right) \cdots \varphi_{B}\left(a_{n}\right)\right\rangle=-m^{-1} \frac{\partial}{\partial\left(-a_{\mu}^{-}\right)} \tau_{B n}, \\
& \left\langle\varphi_{B}\left(a_{1}\right) \cdots \varphi_{-\frac{1}{2},-\frac{1}{2}}^{B}\left(a_{\mu}\right) \cdots \varphi_{B}\left(a_{n}\right)\right\rangle=-m^{-1} \frac{\partial}{\partial a_{\mu}^{+}} \tau_{B n} .
\end{aligned}
$$

Likewise we have

$$
\begin{aligned}
(4.6 .42)_{0} \quad w_{F}^{( \pm)}(y, x) & =\frac{i \tau_{F n}}{\pi}\left\{\begin{array}{l}
\widetilde{w}_{\frac{1}{2}}^{*}[y] \\
-\widetilde{w}_{\frac{1}{2}}[y]
\end{array}\right. \\
& +\sum_{j=-\infty}^{+\infty}\left\langle: \psi_{ \pm}(y) \psi_{\frac{1}{2}+j}(y): \varphi_{F}\left(a_{1}\right) \cdots \varphi_{F}\left(a_{n}\right)\right\rangle w_{\frac{1}{2}+j}[y],
\end{aligned}
$$

(4. 6. 42) ${ }_{\nu} \quad w_{F}^{( \pm)}(y, x)=\frac{1}{2}\left\langle\psi_{ \pm}(y) \varphi_{F}\left(a_{1}\right) \cdots \varphi^{F}\left(a_{\nu}\right) \cdots \varphi_{F}\left(a_{n}\right)\right\rangle$

$$
\begin{aligned}
& \times\left(w_{0}\left[a_{\nu}\right]+w_{0}^{*}\left[a_{\nu}\right]\right) \\
& +\sum_{j=1}^{\infty}\left\langle\psi_{ \pm}(y) \varphi_{F}\left(a_{1}\right) \cdots \varphi_{j}^{F}\left(a_{\nu}\right) \cdots \varphi_{F}\left(a_{n}\right)\right\rangle \cdot w_{j}\left[a_{\nu}\right] \\
& +\sum_{j=1}^{\infty}\left\langle\psi_{ \pm}(y) \varphi_{F}\left(a_{1}\right) \cdots \varphi_{-j}^{F}\left(a_{\nu}\right) \cdots \varphi_{F}\left(a_{n}\right)\right\rangle \cdot w_{j}^{*}\left[a_{\nu}\right] .
\end{aligned}
$$

Here we have set $w_{F}^{( \pm)}(y, x)={ }^{t}\left(w_{F}(y, x)_{ \pm,+}, w_{F}(y, x)_{ \pm,-}\right)$as before.

$$
\begin{aligned}
(4.6 .43)_{\mu \nu} & \pm w_{F \mu}(x)=\frac{1}{2}\left\langle\varphi_{F}\left(a_{1}\right) \cdots \varphi^{F}\left(a_{\mu}\right) \cdots \varphi^{F}\left(a_{\nu}\right) \cdots \varphi_{F}\left(a_{n}\right)\right\rangle \\
& \times\left(w_{0}\left[a_{\nu}\right]+w_{0}^{*}\left[a_{\nu}\right]\right) \\
& +\sum_{j=1}^{\infty}\left\langle\varphi_{F}\left(a_{1}\right) \cdots \varphi^{F}\left(a_{\mu}\right) \cdots \varphi_{j}^{F}\left(a_{\nu}\right) \cdots \varphi_{F}\left(a_{n}\right)\right\rangle \cdot w_{j}\left[a_{\nu}\right] \\
& +\sum_{j=1}^{\infty}\left\langle\varphi_{F}\left(a_{1}\right) \cdots \varphi^{F}\left(a_{\mu}\right) \cdots \varphi_{-j}^{F}\left(a_{\nu}\right) \cdots \varphi_{F}\left(a_{n}\right)\right\rangle \cdot w_{j}^{*}\left[a_{\nu}\right] \\
& \left.-w_{F \mu}(x)=\frac{i \tau_{F n}}{2} w_{0}\left[a_{\mu}\right] \quad \text { (if } \mu \lessgtr \nu\right),
\end{aligned}
$$




$$
\begin{aligned}
& +\sum_{j=1}^{\infty}\left\langle\varphi_{F}\left(a_{1}\right) \cdots \varphi_{F j, 0}\left(a_{\mu}\right) \cdots \varphi_{F}\left(a_{n}\right)\right\rangle w_{j}\left[a_{\mu}\right] \\
& -\frac{i \tau_{F n}}{2} w_{0}^{*}\left[a_{\mu}\right] \\
& +\sum_{j=1}^{\infty}\left\langle\varphi_{F}\left(a_{1}\right) \cdots \varphi_{F-j, 0}\left(a_{\mu}\right) \cdots \varphi_{F}\left(a_{n}\right)\right\rangle w_{j}^{*}\left[a_{\mu}\right]
\end{aligned}
$$

$$
\text { (if } \mu=\nu \text { ). }
$$

(4. 6. 44)

$$
\begin{aligned}
& \left\langle\varphi_{F}\left(a_{1}\right) \cdots \varphi_{F 10}\left(a_{\mu}\right) \cdots \varphi_{F}\left(a_{n}\right)\right\rangle=i m^{-1} \frac{\partial}{\partial\left(-a_{\mu}^{-}\right)} \tau_{F n}, \\
& \left\langle\varphi_{F}\left(a_{1}\right) \cdots \varphi_{F-10}\left(a_{\mu}\right) \cdots \varphi_{F}\left(a_{n}\right)\right\rangle=-i m^{-1} \frac{\partial}{\partial a_{\mu}^{+}} \tau_{F n} .
\end{aligned}
$$

From (4.6.39) $0_{0}-(4.6 .40)_{\mu \nu}$ we see that the Euclidean continuations of

$$
\begin{aligned}
& \widehat{\omega}_{B}(y, x)=\pi w_{B}(y, x) / \tau_{B n}, \\
& \widehat{w}_{B \mu \pm}(x)=2 w_{B \mu \pm}(x) / \tau_{B n}
\end{aligned}
$$

provide the canonical basis $\left\{\widehat{\boldsymbol{w}}_{B}(y, x), \widehat{\boldsymbol{w}}_{B \mu^{+}}(x)(1 \leqq \mu \leqq n)\right\}$ of $W_{B, y}^{0, \frac{1}{2}, \cdots, \frac{1}{2}, \ldots, a_{n}}$, and $\left\{\widehat{\omega}_{B \mu-}(x)(1 \leqq \mu \leqq n)\right\}$ of $W_{B, a_{1}, \cdots, a_{n}}^{* \frac{1}{2}, \cdots, \frac{1}{2}}$ respectively. In particular, from (4.6.40) $)_{\mu \nu}$ and (3.3.11) we obtain expressions for $\tau$ functions involving $\varphi_{ \pm}^{B}(a)$ :

$$
\begin{gathered}
\left\langle\varphi_{B}\left(a_{1}\right) \cdots \varphi_{+}^{B}\left(a_{\mu}\right) \cdots \varphi_{+}^{B}\left(a_{\nu}\right) \cdots \varphi_{B}\left(a_{n}\right)\right\rangle / \tau_{B n}=-f_{\mu \nu} / 2 m\left(a_{\mu}-a_{\nu}\right), \\
\left\langle\varphi_{B}\left(a_{1}\right) \cdots \varphi_{-}^{B}\left(a_{\mu}\right) \cdots \varphi_{-}^{B}\left(a_{\nu}\right) \cdots \varphi_{B}\left(a_{n}\right)\right\rangle / \tau_{B n}=-\bar{f}_{\mu \nu} / 2 m\left(\bar{a}_{\mu}-\bar{a}_{\nu}\right) \\
(\mu \neq \nu),
\end{gathered}
$$

(4. 6. 47) $\left\langle\varphi_{B}\left(a_{1}\right) \cdots \varphi_{T}^{B}\left(a_{\mu}\right) \cdots \varphi_{-}^{B}\left(a_{\nu}\right) \cdots \varphi_{B}\left(a_{n}\right)\right\rangle / \tau_{B n}=-g^{\mu \nu} / 2(\mu \neq \nu)$

where $F=\left(f_{\mu \nu}\right), G=\left(g^{\mu \nu}\right)^{-1}$ denote solutions of (3.3.24) corresponding to $\boldsymbol{v}=\boldsymbol{w}(0)$, and the left hand sides of (4.6.46)-(4.6.47) are identified with their Euclidean continuations.

The situation is somewhat different for the Fermi case. Set

$$
\begin{aligned}
& \widehat{\omega}_{F}(y, x)=\pi i w_{F}(y, x) / \tau_{F n}, \\
& \widehat{\varpi}_{F \mu}(x)=2 i w_{F}(x) / \tau_{F n} .
\end{aligned}
$$

Then the Euclidean continuation of $\widehat{\boldsymbol{w}}_{F_{\mu}}(x)$ belongs to $W_{F, a_{1}, \ldots, a_{n}}^{0, \ldots, 0}$

(*) The definitions of $w_{F \nu}$ and $\hat{\omega}_{F \nu}$ given here differ from [5] by the factors -1 and $-2 i$ respectively. 
$=W_{F, a_{1}, \cdots, a_{n}}^{\text {strict }}$. Their 0 -th coefficient matrices of local expansions $C_{0}=$ $\left(c_{0}^{(\nu)}\left(\widehat{\omega}_{F \mu}\right)\right)_{\mu, \nu=1, \cdots, n}, C_{0}^{*}=\left(c_{0}^{*(\nu)}\left(\widehat{\boldsymbol{w}}_{F_{\mu}}\right)\right)_{\mu, \nu=1, \cdots, n}$ are of the form

$$
C_{0}=1-T, \quad C_{0}^{*}=-1-T
$$

where

$$
T=\left(\begin{array}{ccccc}
0 & -i \hat{\tau}_{F n}^{12} & \cdots \cdots & -i \hat{\tau}_{F n}^{1 n} \\
i \hat{\tau}_{F n}^{21} & 0 & \ddots & \ddots & \vdots \\
\vdots & \ddots & \ddots & \ddots & \vdots \\
\vdots & \ddots & \ddots & -i \hat{\tau}_{F n}^{n-1} n \\
i \hat{\tau}_{F n}^{n 1} & \cdots \cdots & i \hat{\tau}_{F n}^{n n-1} & 0 & 0
\end{array}\right),
$$

$\hat{\tau}_{F n}^{\mu \nu}=\left\langle\varphi_{F}\left(a_{1}\right) \cdots \varphi^{F}\left(a_{\mu}\right) \cdots \varphi^{F}\left(a_{\nu}\right) \cdots \varphi_{F}\left(a_{n}\right)\right\rangle / \tau_{F} \quad(\mu \neq \nu)$. Let $\quad \boldsymbol{w}=\boldsymbol{w}(0)$ $={ }^{t}\left({ }^{t} w_{1}(0), \cdots,{ }^{t} w_{n}(0)\right)$ be the canonical basis of $W_{F, a_{1} \cdots, a_{n}}^{\text {strict }}$, for which we have $C_{0}(\boldsymbol{w})=1$ and $C_{0}^{*}(\boldsymbol{w})=-e^{2 H}=-G^{-1}$ with $H={ }^{t} \bar{H}=-{ }^{t} H$ (see (3.3.7) and Proposition 3.2.5). Since $\widehat{\omega}_{F_{h}}$ is a linear combination of $w_{\nu}(0)$ 's, we have $\widehat{\boldsymbol{w}}_{F}={ }^{t}\left({ }^{t} \widehat{\boldsymbol{w}}_{F 1}, \cdots,{ }^{t} \widehat{\boldsymbol{w}}_{F n}\right)=C \boldsymbol{v}(0)$ with some $C$. Comparing the 0 -th coefficient matrices we obtain $1-T=C,-1-T=C\left(-e^{2 H}\right)$. Since $1+e^{2 H}$ is invertible, this implies

$$
T=\tanh H=(1-G)(1+G)^{-1} .
$$

In particular $C=e^{-H}(\cosh H)^{-1}=2 G(1+G)^{-1}$ is invertible, so that $\widehat{\boldsymbol{w}}_{F^{\prime} \mu}$ $(1 \leqq \mu \leqq n)$ is also a basis of $W_{F, a_{1}, \cdots, a_{n}}^{\text {strict }}$.

Proposition 4.6.5. The logarithmic derivative of $\tau$ functions are given by

$$
\begin{aligned}
& d \log \tau_{B n}=-\frac{1}{2} \omega \\
& d \log \tau_{F n}=\frac{1}{4} \operatorname{trace}\left(T \Theta+\Theta^{*} T\right)+\frac{1}{2} \omega
\end{aligned}
$$

where $\omega$ is the 1-form (3.3.57) associated with the system (3.2.24) corresponding to $w=w(0)$, and $T$ is given by (4.6.51). Moreover we have

$$
\tau_{B n} \cdot \tau_{F n}=\sqrt{\operatorname{det} \cosh H}
$$

Proof. Expression for $d \log \tau_{B n}$ follows from (4.6.41). We con- 
sider $d \log \tau_{\Gamma n}$. Denoting the 1 -st coefficient matrices of local expansions by $C_{1}\left(\widehat{\omega}_{F}\right)$ and $C_{1}^{*}\left(\widehat{\boldsymbol{w}}_{F}\right)$, we have from (4.6.44)

(4. 6. 54) $\quad d \log \tau_{F n}=\frac{1}{2} \operatorname{trace}\left(C_{1}\left(\widehat{\varpi}_{F}\right) d A-C_{1}^{*}\left(\widehat{\varpi}_{F}\right) d \bar{A}\right)$

in the Euclidean region. On the other hand, the relation $\widehat{w}_{F}=(1-T)$ $\times \mathfrak{v}(0)$ implies that $C_{1}\left(\widehat{\boldsymbol{w}}_{F}\right)=(1-T) \alpha$ and $C_{1}^{*}\left(\widehat{\boldsymbol{w}}_{F}\right)=(1-T) \beta \bar{\alpha}=-(1$ $+T) \bar{\alpha}$ by (3.3.7), with $\alpha=\alpha(1 / 2)={ }^{t} \alpha$. Combining this with (4.6.54) and noting that trace $T \alpha d A=\operatorname{trace} d A^{t} \alpha^{t} T=-\operatorname{trace} T d A \cdot \alpha=$ $\frac{1}{2} \operatorname{trace} T[\alpha, d A]=-\frac{1}{2}$ trace $T \Theta$, we obtain (4.6.52).

To prove (4.6.53), we calculate the $d \log$ of the right hand side.

(4. 6. 55) $\quad d \log \sqrt{\operatorname{det} \cosh H}=\frac{1}{2} d \operatorname{trace}(\log \cosh H)$

$$
\begin{aligned}
& =\frac{1}{2} \operatorname{trace}(\tanh H \cdot d H) \\
& =\frac{1}{2} \operatorname{trace}\left(T \cdot\left(-\frac{1}{2}\right) G^{-1} d G\right) .
\end{aligned}
$$

Making use of the differential equations (3.3.24), we have

$$
\text { (4.6.56) } \begin{aligned}
\operatorname{trace}\left(T\left(-\frac{1}{2}\right) G^{-1} d G\right) & =\frac{1}{2} \operatorname{trace}\left(T \Theta+T G^{-1} \Theta * G\right) \\
& =\frac{1}{2} \operatorname{trace}\left(T \Theta+\Theta^{*} T\right)
\end{aligned}
$$

since $T$ commutes with $G$. Now (4.6.55), (4.6.56) and (4.6.52) imply

$$
d \log \tau_{B n}+d \log \tau_{F n}=d \log \sqrt{\operatorname{det} \cosh H} .
$$

Since $\tau_{B n}, \tau_{F_{n}} \rightarrow 1$ and $H \rightarrow 0$ as $\left|a_{\mu}-a_{\nu}\right| \rightarrow \infty$ for all $\mu \neq \nu$, we obtain (4. 6. 53). This proves Proposition 4.6.5.

$\tau$ functions involving $\varphi_{ \pm}^{B}(a)$ or $\varphi^{F}(a)$ are obtained by applying (A. 27) or its orthogonal version. Set

$$
\begin{array}{ll}
\text { (4. 6. 57) } \quad \hat{\tau}_{B n ; \varepsilon_{1}, \cdots, \varepsilon_{m}}^{\nu_{1}, \cdots, \nu_{m}}=\left\langle\varphi_{B}\left(a_{1}\right) \cdots \varphi_{\varepsilon_{1}}^{B}\left(a_{\nu_{1}}\right) \cdots \varphi_{\varepsilon_{m}}^{B}\left(a_{\nu_{m}}\right) \cdots \varphi_{B}\left(a_{n}\right)\right\rangle / \tau_{B n} \\
\\
\hat{\tau}_{F n}^{\nu_{1}, \cdots, \nu_{m}}=\left\langle\varphi_{F}\left(a_{1}\right) \cdots \varphi^{F}\left(a_{\nu_{1}}\right) \cdots \varphi^{F}\left(a_{\nu_{m}}\right) \cdots \varphi_{F}\left(a_{n}\right)\right\rangle / \tau_{F n} .
\end{array}
$$

Here $\varphi_{\varepsilon_{i}}^{B}\left(a_{\nu_{i}}\right)\left(\operatorname{resp} . \varphi^{F}\left(a_{\nu_{i}}\right)\right)$ are placed in the $\nu_{i}$ th position for $i=1, \cdots, m$. 
From (4.6.46), (4.6.47), (4.6.50) and (4.6.51) we have then

$$
\begin{aligned}
& \hat{\tau}_{B n ; \varepsilon_{1}, \cdots, \varepsilon_{m}}^{\nu, \ldots, \nu_{m}}=\text { Hafnian }\left(\hat{\tau}_{B n ; \varepsilon_{j} \varepsilon_{k}}^{\nu, \nu_{k}}\right)_{j, k=1, \ldots, m}, \\
& \hat{\tau}_{B n ;++}^{\mu \nu}=\overline{\hat{\tau}_{B n ;--}^{\mu \nu}}=-f_{\mu \nu} / 2 m\left(a_{\mu}-a_{\nu}\right) \quad(\mu \neq \nu), \\
& \hat{\tau}_{B n ;+-}^{\mu \nu}=\overline{\hat{\tau}_{B n ;-+}^{\mu \nu}}=-g^{\mu \nu} / 2 \quad(\mu \neq \nu),
\end{aligned}
$$

(4.6.59) $\quad \hat{\tau}_{F, n}^{\nu_{1}, \cdots, \nu_{m}}=\operatorname{Pfaffian}\left(i(\tanh H)_{\nu_{j} \nu_{k}}\right)_{j, k=1, \ldots, m}$.

In particular the 2-point functions are given in terms of $\psi(t)=\psi_{0}(t ; 1)$ in (4.5.36) (with $\tilde{\lambda}=1$ ) as follows. We set $a_{1}-a_{2}=t e^{i \theta} / 2 m, t>0$.

(4. 6. 60) $\left\langle\varphi_{B}\left(a_{1}\right) \varphi_{B}\left(a_{2}\right)\right\rangle$

$$
\begin{gathered}
=\exp \left\{-\frac{1}{4} \int_{\infty}^{t} s d s\left(\left(\frac{d \psi}{d s}(s)\right)^{2}-\sinh ^{2} \psi(s)\right)\right\}, \\
\left(\begin{array}{cc}
\left\langle\varphi_{+}^{B}\left(a_{1}\right) \varphi_{+}^{B}\left(a_{2}\right)\right\rangle\left\langle\varphi_{+}^{B}\left(a_{1}\right) \varphi_{-}^{B}\left(a_{2}\right)\right\rangle \\
\left\langle\varphi_{-}^{B}\left(a_{1}\right) \varphi_{+}^{B}\left(a_{2}\right)\right\rangle\left\langle\varphi_{-}^{B}\left(a_{1}\right) \varphi_{-}^{B}\left(a_{2}\right)\right\rangle
\end{array}\right) \\
=\left(\begin{array}{cc}
-i e^{-i \theta} \frac{d \psi}{d t}(t) & -i \sinh \psi(t) \\
i \sinh \psi(t) & i e^{i \theta} \frac{d \psi}{d t}(t)
\end{array}\right) \cdot\left\langle\varphi_{B}\left(a_{1}\right) \varphi_{B}\left(a_{2}\right)\right\rangle / 2 .
\end{gathered}
$$

(4. 6. 61) $\left\langle\varphi_{F}\left(a_{1}\right) \varphi_{F}\left(a_{2}\right)\right\rangle$

$$
\begin{gathered}
=\cosh \frac{\phi(t)}{2} \exp \left\{\frac{1}{4} \int_{\infty}^{t} s d s\left(\left(\frac{d \psi}{d s}(s)\right)^{2}-\sinh ^{2} \psi(s)\right)\right\}, \\
\left\langle\varphi^{F}\left(a_{1}\right) \varphi^{F}\left(a_{2}\right)\right\rangle=\tanh \frac{\psi(t)}{2} \cdot\left\langle\varphi_{F}\left(a_{1}\right) \varphi_{F}\left(a_{2}\right)\right\rangle .
\end{gathered}
$$

Combining this with the result in [12] we have the following short distance behavior:

$$
\begin{aligned}
& \left\langle\varphi_{B}\left(a_{1}\right) \varphi_{B}\left(a_{2}\right)\right\rangle \sim \text { const. } t^{-\frac{1}{4}}\left(\log \frac{1}{t}\right)^{-\frac{1}{2}} \\
& \left\langle\varphi_{+}^{B}\left(a_{1}\right) \varphi_{+}^{B}\left(a_{2}\right)\right\rangle \sim \text { const. } t^{-\frac{5}{4}}\left(\log \frac{1}{t}\right)^{-\frac{1}{2}} \quad(t \rightarrow 0) \\
& \left\langle\varphi_{+}^{B}\left(a_{1}\right) \varphi_{-}^{B}\left(a_{2}\right)\right\rangle \sim \text { const. } t^{-\frac{5}{4}}\left(\log \frac{1}{t}\right)^{-\frac{3}{2}} .
\end{aligned}
$$

Compare with the result of [11] which states 
(4. 6. 63) $\left\langle\varphi_{F}\left(a_{1}\right) \varphi_{F}\left(a_{2}\right)\right\rangle,\left\langle\varphi^{F}\left(a_{1}\right) \varphi^{F}\left(a_{2}\right)\right\rangle \sim$ const. $t^{-1 / 4}$.

Infinite series expressions for these $\tau$ functions are also avaulable. In the bosonic case they are almost the same as in the previous section. Set

$$
\begin{aligned}
& E_{B, 0}(u)=\left(e_{\mu \nu, 0}(u)\right)_{\mu, \nu=1, \ldots, n}, \\
& e_{\mu \nu, 0}(u)=-\left|\varepsilon_{\mu \nu}\right| \cdot \theta\left(-\varepsilon_{\mu \nu} u\right) e^{-i m\left\{\left(a_{\mu}-a_{\nu}\right)-u+\left(a_{\mu}-a_{\nu}\right)+u-1\right\}} .
\end{aligned}
$$

Then

(4. 6. 65) $\log \tau_{B n}=\sum_{l=2}^{\infty} \frac{1}{2 l} \int \cdots \int \frac{d u_{1}}{2 \pi} \cdots \frac{d u_{l}}{2 \pi} \frac{2 i}{u_{1}-u_{2}+i 0} \frac{2 i}{u_{2}-u_{3}+i 0} \cdots$

$$
\cdots \frac{2 i}{u_{l}-u_{1}+i 0} \cdot \operatorname{trace}\left(E_{B, 0}\left(u_{1}\right) E_{B, 0}\left(u_{2}\right) \cdots E_{B, 0}\left(u_{l}\right)\right) .
$$

Formula (4.6.65) is exactly the one half of (4.5.7) with $\lambda_{\mu \nu}=1$ and $l_{\mu}=1 / 2(\mu, \nu=1, \cdots, n)$. We have further

$$
\text { (4.6.66) } \begin{aligned}
\hat{\tau}_{B ;++}^{\mu \nu}=- & \sum_{l=0}^{\infty} \int \cdots \int \frac{d u_{1}}{2 \pi} \cdots \frac{d u_{l+1}}{2 \pi} \frac{2 i}{u_{1}-u_{2}+i 0} \frac{2 i}{u_{2}-u_{3}+i 0} \cdots \\
& \cdots \frac{2 i}{u_{l}-u_{l+1}+i 0}\left[E_{B, 0}\left(u_{1}\right) E_{B, 0}\left(u_{2}\right) \cdots E_{B, 0}\left(u_{l+1}\right)\right]_{\mu \nu}, \\
\hat{\tau}_{B ;+-}^{\mu \nu}=- & i \sum_{i=0}^{\infty} \int \cdots \int \frac{d u_{1}}{2 \pi} \cdots \frac{d u_{l+1}}{2 \pi} \frac{2 i}{u_{1}-u_{2}+i 0} \frac{2 i}{u_{2}-u_{3}+i 0} \cdots \\
& \cdots \frac{2 i}{u_{l}-u_{l+1}+i 0} \frac{1}{u_{l+1}}\left[E_{B, 0}\left(u_{1}\right) E_{B, 0}\left(u_{2}\right) \cdots E_{B, 0}\left(u_{l+1}\right)\right]_{\mu \nu}
\end{aligned}
$$

where $[\cdots]_{\mu \nu}$ signifies the $(\mu, \nu)$-th matrix element. For $n=2$ these formulas read in the Euclidean region

$$
\log \tau_{B 2}=\sum_{k=1}^{\infty} \frac{2^{2 k}}{2 k} e_{0}^{(2 k)}(t),
$$

$$
\begin{aligned}
& \hat{\tau}_{B ;++}^{12}=i e^{-i \theta} f_{0}(t ; 1) / 2, \\
& \hat{\tau}_{B ;+-}^{12}=i h_{0}(t ; 1) / 2
\end{aligned}
$$

where $-\left(a_{1}^{-}-a_{2}^{-}\right)=t e^{i \theta} / 2 m, a_{1}^{+}-a_{2}^{+}=t e^{-i \theta} / 2 m$, and $e^{(2 k)}(t), f_{0}(t ; 1)$, $h_{0}(t ; 1)$ denote those in $(4.5 .31),(4.5 .33)-(4.5 .34)$ respectively.

In the fermionic case we set 


$$
E_{F, 0}(u)=\left(-\varepsilon_{\mu \nu} e_{\mu \nu, 0}(u)\right)_{\mu, \nu=1, \ldots, n} .
$$

We have then (cf. (4.6.59))

(4.6. 70) $\log \tau_{F n}=-\sum_{l=2}^{\infty} \frac{1}{2 l} \int \cdots \int d u_{1} \cdots d u_{l} \frac{i\left(u_{1}+u_{2}\right)}{u_{1}-u_{2}+i 0} \frac{i\left(u_{2}+u_{3}\right)}{u_{2}-u_{3}+i 0} \cdots$

$$
\cdots \frac{i\left(u_{l}+u_{1}\right)}{u_{l}-u_{1}+i 0} \operatorname{trace}\left(E_{F, 0}(u) \cdots E_{F, 0}\left(u_{l}\right)\right) \text {, }
$$

(4.6.71) $i \tanh H=-\sum_{i=0}^{\infty} \int \cdots \int d u_{1} \cdots d u_{l+1} \frac{i\left(u_{1}+u_{2}\right)}{u_{1}-u_{2}+i 0} \frac{i\left(u_{2}+u_{3}\right)}{u_{2}-u_{3}+i 0} \cdots$

$$
\cdots \frac{i\left(u_{l}+u_{l+1}\right)}{u_{l}-u_{l+1}+i 0} E_{F, 0}\left(u_{1}\right) \cdots E_{F, 0}\left(u_{l+1}\right)
$$

with $d u=d u / 2 \pi|u|$. For $n=2$ they reduce to

(4.6. 72) $\log \tau_{F 2}=-\sum_{k=1}^{\infty} \frac{1}{2 k} \int_{0}^{\infty} \cdots \int \underline{d u_{1}} \cdots \underline{d u_{2 k}} \frac{u_{1}-u_{2}}{u_{1}+\iota_{2}} \frac{u_{2}-u_{3}}{u_{2}+u_{3}} \cdots \frac{u_{2 k}-u_{1}}{u_{2 k}+u_{1}}$

$$
\times \exp \left(-\frac{t}{2}\left(U_{2 k}+U_{2 k}^{\prime}\right)\right)
$$

(4. 6. 73) $\hat{\tau}_{F}^{12}=\sum_{k=0}^{\infty} \int_{0}^{\infty} \cdots \int \underline{d u_{1}} \cdots \underline{d u_{2 k+1}} \frac{u_{1}-u_{2}}{u_{1}+u_{2}} \frac{u_{2}-u_{3}}{u_{2}+u_{3}} \cdots \frac{u_{2 k}-u_{2 k+1}}{u_{2 k}+u_{2 k+1}}$

$$
\times \exp \left(-\frac{t}{2}\left(U_{2 k+1}+U_{2 k+1}^{\prime}\right)\right)
$$

respectively, where $t=2 m \sqrt{-\left(a_{1}^{-}-a_{2}^{-}\right)\left(a_{1}^{+}-a_{2}^{+}\right)}$and $U_{l}=u_{1}+\cdots+u_{l}$, $U_{l}^{\prime}=u_{1}^{-1}+\cdots+u_{l}^{-1}$.

These expressions (4.6.70)-(4.6.73) are in agreement with those for the scaled $n$-point functions of the 2-dimensional Ising model [11], [13].

\section{Appendix}

We summarize here generalities on norms and rotations in a symplectic vector space.

A symplectic vector space $W$ is by definition a vector space over $\mathbb{C}$ equipped with an anti-symmetric inner product $\langle$,$\rangle . Throughout this$ 
appendix we assume $N=\operatorname{dim}_{\boldsymbol{C}} W$ to be finite. ${ }^{(*)}$ Infinite dimensional case is dealt with similarly as in the orthogonal case (see Section 2.1).

Let $T(W)$ be the contravariant tensor algebra over $W$, and let $I(W)$ denote the bi-ideal of $T(W)$ generated by elements of the form $w \otimes w^{\prime}$ $-w^{\prime} \otimes w-\left\langle w, w^{\prime}\right\rangle \quad\left(w, w^{\prime} \in W\right)$. We set $A(W)=T(W) / I(W)$. In contrast with the orthogonal case, $\operatorname{dim}_{\boldsymbol{C}} A(W)=\infty$ even if $W$ is finite dimensional. We denote by $S\left(W^{\prime}\right)$ the symmetric tensor algebra over $W$. Let $\kappa$ be an element of $\operatorname{Hom}_{\boldsymbol{C}}\left(W, W^{*}\right)$. The bilinear form $W \times W$ $\rightarrow \boldsymbol{C}, \quad\left(w, w^{\prime}\right) \mapsto \kappa(w)\left(w^{\prime}\right)$ is called an expectation value if $\kappa(w)\left(w^{\prime}\right)$ $-\kappa\left(w^{\prime}\right)(w)=\left\langle w, w^{\prime}\right\rangle$ holds for all $w, w^{\prime} \in W$. Given an expectation value, the associated norm map $\mathrm{Nr}_{\kappa}$ is defined as in the orthogonal case.

Denote by $\delta$ the linear map $W^{*} \rightarrow \operatorname{End}_{\boldsymbol{C}}(S(W))$ such that each $\delta\left(w^{*}\right)$ is a derivation: $\delta\left(w^{*}\right)(1)=0, \delta\left(w^{*}\right)(w \cdot a)=w^{*}(w) \cdot a+w \cdot \delta$ $\left(w^{*}\right)(a) \quad\left(w^{*} \in W^{*}, w \in W, a \in S(W)\right)$. Then there exists a unique linear isomorphism

$$
\mathrm{Nr}_{\kappa}: A(W) \rightarrow S(W), \quad \mathrm{Nr}_{\kappa}(1)=1
$$

characterized by either of the following properties:

(i) $\mathrm{Nr}_{\kappa}(w a)=w \mathrm{Nr}_{\kappa}(a)+\delta_{w}\left(\mathrm{Nr}_{\kappa}(a)\right) \quad(w \in W, a \in A(W))$

(ii) $\mathrm{Nr}_{\kappa}(a w)=\mathrm{Nr}_{\kappa}(a) \cdot w+\delta_{w}^{\prime}\left(\mathrm{Nr}_{\kappa}(a)\right) \quad(w \in W, a \in A(W))$

where $\delta_{w}=\delta(\kappa(w)), \delta_{w}^{\prime}=\delta\left({ }^{t} \kappa(w)\right) \quad(w \in W)$. The term of degree 0 of $\mathrm{Nr}_{\kappa}(a)$ in the graded algebra $S(W)=\boldsymbol{C} \oplus W \oplus S^{2}(W) \oplus \cdots$ is also called the expectation value of $a$ and is denoted by $\langle a\rangle_{\kappa}$. Note that $\left\langle w \cdot w^{\prime}\right\rangle_{\kappa}$ $=\kappa(w)\left(w^{\prime}\right) \quad\left(w, w^{\prime} \in W\right)$. For notational simplicity we often drop $\kappa$. An element of $A(W)$ (resp. $S(W)$ ) is called an operator (resp. a norm). Denoting the inverse map $\mathrm{Nr}^{-1}$ by : :, we have the following formulas:

$$
\begin{aligned}
& : w_{1} \cdots w_{k}:: w_{1}^{\prime} \cdots w_{l}^{\prime}: \\
& \quad=\sum\left\langle w_{\mu_{1}} w_{\nu_{\sigma(1)}}^{\prime}\right\rangle \cdots\left\langle w_{\mu_{m}} w_{\nu_{\sigma(m)}}^{\prime}\right\rangle: w_{\mu_{1}^{\prime}} \cdots w_{\mu_{k-m}^{\prime}} w_{\nu_{1}^{\prime}}^{\prime} \cdots w_{\nu_{l-m}^{\prime}}^{\prime}: .
\end{aligned}
$$

Here the sum is taken over all the partitions $\left\{\mu_{1}, \cdots, \mu_{m}\right\} \sqcup\left\{\mu_{1}^{\prime}, \cdots, \mu_{k-m}^{\prime}\right\}$ $=\{1, \cdots, k\} \quad\left(\mu_{1}<\cdots<\mu_{m}, \mu_{1}^{\prime}<\cdots<\mu_{k_{-}-m}^{\prime}\right), \quad\left\{\nu_{1}, \cdots, \nu_{m}\right\} \sqcup\left\{\nu^{\prime}, \cdots, \nu_{l-m}^{\prime}\right\}=\{1$, $\cdots, l\} \quad\left(\nu_{1}<\cdots<\nu_{m}, \nu_{1}^{\prime}<\cdots<\nu_{l-m}^{\prime}\right)$ and $\sigma \in \Im_{m}$.

\footnotetext{
(*) We do not assume the inner product to be non-degenerate, unless otherwise stated.
} 
(A. 4)

$$
: w_{1} \cdots w_{k}:=\sum(-)^{m}\left\langle w_{\mu_{1}} w_{\mu_{2}}\right\rangle \cdots\left\langle w_{\mu_{2 m-1}} w_{\mu_{2 m}}\right\rangle w_{\nu_{1}} \cdots w_{\nu_{k-2 m}} \text {, }
$$

(A. 5) $\quad \operatorname{Nr}\left(w_{1} \cdots w_{k}\right)=\sum\left\langle w_{\mu_{1}} w_{\mu_{2}}\right\rangle \cdots\left\langle w_{\mu_{2 m-1}} w_{\mu_{2 m}}\right\rangle w_{\nu_{1}} \cdots w_{\nu_{k-2 m}}$

where the sum is taken over all the partitions $\left\{\mu_{1}, \cdots, \mu_{2 m}\right\} \sqcup\left\{\nu_{1}, \cdots\right.$, $\left.\nu_{k-2 m}\right\}=\{1, \cdots, k\} \quad\left(\mu_{1}<\mu_{3}<\cdots<\mu_{2 m-1}, \mu_{1}<\mu_{2}, \cdots, \mu_{2 m-1}<\mu_{2 m}, \quad \nu_{1}<\cdots\right.$ $\left(\nu_{k-2 m}\right)$.

An important example of an expectation value \langle\rangle$_{\kappa}$ is provided by a holonomic decomposition $\left.W=V^{*} \oplus V .^{*}\right)$ Here $V^{*}, V$ denote holonomic subspaces of $W$ in the sense that $\left\langle v, v^{\prime}\right\rangle=0$ for any $v, v^{\prime} \in V,\left\langle v^{*}, v^{* \prime}\right\rangle$ $=0$ for any $v^{*}, v^{* \prime} \in V^{*}$. We have then $A(W)=S\left(V^{*}\right) \cdot S(V)$ and $S(W)=S\left(V^{*}\right) \cdot S(V)$. We define the expectation value by $\left\langle\left(v+v^{*}\right)\right.$ $\left.\cdot\left(v^{\prime}+v^{* \prime}\right)\right\rangle=\left\langle v, v^{* \prime}\right\rangle\left(v, v^{\prime} \in V, v^{*}, v^{* \prime} \in V^{*}\right)$. The corresponding norm map (A. 1) is then a $\left(S\left(V^{*}\right), S(V)\right)$-isomorphism. Take a basis $v_{j}^{*} \in V^{*}$, $v_{j} \in V(j=1, \cdots, r, N=2 r)$ so that

$$
K=\left(\begin{array}{ll}
\left\langle v_{j}^{*} v_{k}^{*}\right\rangle & \left\langle v_{j}^{*} v_{k}\right\rangle \\
\left\langle v_{j} v_{k}^{*}\right\rangle & \left\langle v_{j} v_{k}\right\rangle
\end{array}\right)_{j, k=1, \ldots, r}=\left(\begin{array}{c}
0 \\
1
\end{array}\right) .
$$

Then $A(W)$ is isomorphic to the algebra of differential operators with polynomial coefficients $C\left[x_{1}, \cdots, x_{r}, \frac{\partial}{\partial x_{1}}, \cdots, \frac{\partial}{\partial x_{r}}\right]$ by the identification $v_{j}^{*}$ $=x_{j}, v_{j}=\frac{\partial}{\partial x_{j}}$. The norm of $P\left(x, \frac{\partial}{\partial x}\right)$ is usually called the total symbol of $P$. The vacuums $|v a c\rangle,\langle v a c|$ are defined in just the same way as in the orthogonal case.

Let $\mathbb{C}[[t]], A(W)[[t]]$ and $S(W)[[t]]$ denote the algebras of formal power series in $t$ with coefficients in $C, A(W)$ and $S(W)$ respectively. We call an element of $A(W)[[t]]$ (resp. $S(W)[[t]]$ ) an operator (resp. a norm), also. When we fix an expectation value in $W, \mathbb{C}$-linear isomorphisms $\mathrm{Nr}$ and : : are uniquely extended to $\mathbb{C}[[t]]$-linear isomorphisms between $A(W)[[t]]$ and $S(W)[[t]]$, which we denote also by $\mathrm{Nr}$ and : : respectively. We set $S(W)[[t]]^{f}=\{a \in S(W)[[t]] \mid a$ $\in \bigoplus_{j=0}^{k} S^{j}(W) \underset{\boldsymbol{C}}{\otimes} \boldsymbol{C}[[t]]$ for some $\left.k \in \mathbb{N}\right\}$. Then $A(W)[[t]]_{\text {def }}^{f}=\{a \in$ $\left.A(W)[[t]] \mid \operatorname{Nr}(a) \in S(W)[[t]]^{f}\right\}$ is independent of the choice of expectation values.

Denoting by $\mathfrak{m}$ the maximal ideal $C[[t]] t$, we set

(*) We assume that the inner product is non-degenerate, hence $W$ is even dimensional. 
(A. 6) $G(W, t)=\left\{g \in A(W)[[t]] \mid \operatorname{Nr}(g)=\langle g\rangle e^{\rho / 2}\right.$,

$$
\left.\langle g\rangle \in \mathbb{C}[[t]]-\mathfrak{m}, \rho \in S^{2}(W) \underset{\boldsymbol{C}}{\otimes} \mathfrak{m}\right\},
$$

(A. 7) $\widetilde{G}(W, t)=\left\{g \in A(W)[[t]] \mid \operatorname{Nr}(g)=a e^{o / 2}\right.$,

$$
\left.a \in S(W)[[t]]^{f}, \rho \in S^{2}(W) \underset{\boldsymbol{C}}{\otimes} \mathfrak{m}\right\}
$$

It is shown below that $G(W, t)$ (resp. $\widetilde{G}(W, t)$ ) forms a group (resp. a semi-group) independent of the choice of expectation values.

Let $v_{1}, \cdots, v_{N}$ be a basis of $W$, and set

$$
\begin{aligned}
& K=\left(\left\langle v_{j} v_{k}\right\rangle\right)_{j, k=1, \cdots, N}, \\
& H=K-{ }^{t} K, J=K+{ }^{t} K .
\end{aligned}
$$

The product of $g \in \widetilde{G}(W, t)$ and an element $a \in A(W)[[t]]^{f}$ is given as follows. Without loss of generality we assume that $g$ is of the form (A. 9) $\quad \operatorname{Nr}(g)=c w_{1} \cdots w_{s} e^{\rho / 2}$,

$$
c \in C[[t]], w_{l}=\sum_{j=1}^{N} v_{j} c_{j}^{(l)}(l=1, \cdots, s), \rho=\sum_{j, k=1}^{N} r_{j k} v_{j} v_{k}
$$

where $c_{j}^{(l)} \in C[[t]] \quad(l=1, \cdots, s ; j=1, \cdots, N) \quad$ and $\quad r_{j k} \in \mathfrak{m}, r_{j k}=r_{k j}$, and that $a=\sum_{j=1}^{N} v_{j} c_{j}=w$ where $c_{j} \in C[[t]]$. Applying (A.3) we have

$$
\operatorname{Nr}(w g)=\left(\sum_{l=1}^{s} w_{1} \cdots\left\langle w w_{l}\right\rangle \cdots w_{s}+w^{(1)} w_{1} \cdots w_{s}\right) e^{\rho / 2}
$$

where $w^{(1)}=\sum_{j, k=1}^{N} v_{j}\left(1+R^{t} K\right)_{j k} c_{k}$,

$$
\operatorname{Nr}(g w)=\left(\sum_{l=1}^{s} w_{1} \cdots\left\langle w_{l} w\right\rangle \cdots w_{s}+w_{1} \cdots w_{s} w^{(2)}\right) e^{\rho / 2}
$$

where $w^{(2)}=\sum_{j, k=1}^{N} v_{j}(1+R K)_{j k} c_{k}$.

Now we assume $s=0$, so that we have $c=\langle g\rangle$. Noting that 1 $+R^{t} K$ is invertible, we define $T=\left(t_{j k}\right)_{j, k=1, \cdots, N}$ by

$$
\left(1+R^{t} K\right) T=1+R K .
$$

Then (A. 10) and (A. 11) imply

$$
g v_{k}=\sum_{j=1}^{N} v_{j} g t_{j k} \quad(k=1, \cdots, N) .
$$

(A. 12) is rewritten as 


$$
R\left(K-{ }^{t} K T\right)=T-1
$$

Proposition A. 1. If $R=\left(r_{j k}\right)_{j, k=1, \ldots, N}$ is a symmetric matrix such that $r_{j k} \in \mathfrak{m}$, then $T=\left(1+R^{t} K\right)^{-1}(1+R K)$ is symplectic in the sense

$$
{ }^{t} T H T=H,
$$

and moreover

$$
t_{j k}-\delta_{j k} \in \mathrm{m} \mathrm{l} .
$$

Now we assume that $H$ is invertible. If $T=\left(t_{j k}\right)_{j, k=1, \ldots, N}$ satisfy (A. 15) and (A. 16), then $K-{ }^{t} K T$ is invertible, $R=(T-1)\left(K-{ }^{t} K T\right)^{-1}$ is symmetric and $r_{j k} \in \mathrm{m}$.

The proof is not so difficult.

We remark here that, contrary to the orthogonal case, an element $w \in W$ never induces a non-trivial rotation in $W$. To see this observe that if $w_{1} w_{2}=c$ for $w_{1}, w_{2} \in W, c \in C$, then $c=0$ and either $w_{1}=0$ or $w_{2}=0$. Now let $w_{0} \in W, T \in \operatorname{End}(W)$ satisfy $w_{0} w=(T w) w_{0}$ for all $w$ $\in W$. We have then $(T w-w) w_{0}=\left[w_{0}, w\right]$, so that $w_{0}=0$ or $T w=w$, $\left[w_{0}, w\right]=0$ for all $w \in W$, i.e. $T=1$.

The following proposition is sometimes useful in order to obtain $R$ from $T$.

Proposition A. 2. We assume that $H$ is invertible and set

$$
E^{-1}=H^{-1} J, \quad P=(1-T) / 2 .
$$

Suppose there exist $X_{ \pm}$that are invertible and satisfy

$$
\text { (A. 18) }{ }_{B} \quad P X_{+}(1-P)=0, \quad(1-P) X_{-} P=0, \quad X_{-}=X_{+} E^{-1} \text {. }
$$

Then the following $R$ satisfies (A.14).

$$
\text { (A. 19) }{ }_{B} \quad R=-2 X_{-}^{-1} P X_{+} H^{-1} \text {. }
$$

$$
\text { Proof. } \begin{aligned}
R\left(K-{ }^{t} K T\right) & =-2 X_{-}^{-1} P X_{+}\left(H^{-1} K-H^{-1}{ }^{t} K T\right) \\
& =-2 X_{-}^{-1} P X_{+}\left(\frac{1+E^{-1}}{2}+\frac{1-E^{-1}}{2} T\right) \\
& =-2 X_{-}^{-1} P X_{+}\left(1-P+E^{-1} P\right)
\end{aligned}
$$




$$
=-2 X_{-}^{-1} P X_{-} P=-2 P=T-1 \text {. }
$$

In the orthogonal case, if we modify $\left(\right.$ A. 18) ${ }_{B}$ so that (A. 18) $F \quad P X_{+}(1-P)=0, \quad(1-P) X_{-} P=0, \quad X_{-}=X_{+} E$, $R$ is given by
(A. 19) $F$
$R=-2 X_{-}^{-1} P X_{+} J^{-1}$

Remark. The condition (A. 18) ${ }_{B}$ ensures the left invertibility of $K-{ }^{t} K T$. Indeed a similar calculation shows that

$$
\left(X_{+}^{-1}(1-P)+X_{-}^{-1} P\right) X_{+} H^{-1} \cdot\left(K-{ }^{t} K T\right)=1 .
$$

If we assume further the condition

$$
\text { (A. 18) }{ }_{B}^{\prime} \quad P X_{+}^{-1}(1-P)=0, \quad(1-P) X_{-}^{-1} P=0
$$

then $\left(X_{+}^{-1}(1-P)+X_{-}^{-1} P\right) X_{+} H^{-1}$ is also the right inverse of $K-{ }^{t} K T$. Similarly in the orthogonal case (A. 18) $F$ implies

$$
\left(X_{+}^{-1}(1-P)+X_{-}^{-1} P\right) X_{+} J^{-1} \cdot\left(K+{ }^{t} K T\right)=1,
$$

while

$$
\text { (A. 18) }{ }_{F}^{\prime} \quad P X_{+}^{-1}(1-P)=0, \quad(1-P) X_{-}^{-1} P=0
$$

guarantees

$$
\left(K+{ }^{t} K T\right) \cdot\left(X_{+}^{-1}(1-P)+X_{-}^{-1} P\right) X_{+} J^{-1}=1 .
$$

Now we investigate the symplectic analogues of the transformation rule $(1.5 .20)-(1.5 .21)$ and the product formula (1.4.6)-(1.4.7) in the orthogonal case. Most of the formulas are obtained by replacing ${ }^{t} K, H$, $J$ and $\operatorname{det}$ by $-{ }^{t} K, J, H$ and $\operatorname{det}^{-1}$, respectively, in the orthogonal version.

Let \langle\rangle$^{\prime}$ be another expectation value, and let $\mathrm{Nr}^{\prime},::^{\prime}$, etc. denote the corresponding objects. We have then

$$
\begin{aligned}
\langle g\rangle^{\prime} & =\langle g\rangle \operatorname{det}\left(1-\left(K^{\prime}-K\right) R\right)^{-\frac{1}{2}}, \\
& =\langle g\rangle \exp \frac{1}{2 l} \sum_{l=1}^{\infty} \operatorname{trace}\left\{\left(K^{\prime}-K\right) R\right\}^{l},
\end{aligned}
$$

$$
\mathrm{Nr}^{\prime}(g)=\langle g\rangle^{\prime} \exp \left(\rho^{\prime} / 2\right)
$$


where

$$
\begin{aligned}
\rho^{\prime}=\sum_{j, k=1}^{N} r_{j k}^{\prime} v_{j} v_{k}, R^{\prime} & =\left(r_{j k}^{\prime}\right)=R\left(1-\left(K^{\prime}-K\right) R\right)^{-1} \\
& =\sum_{l=0}^{\infty} R\left\{\left(K^{\prime}-K\right) R\right\}^{l}
\end{aligned}
$$

Proof. We shall prove (A. 16). (A. 17) is proved similarly. Using (A. 5), we have

$$
\begin{aligned}
g /\langle g\rangle= & +\frac{1}{2} \sum_{j, k=1}^{N} r_{j k}\left(v_{j} v_{k}-\left\langle v_{j} v_{k}\right\rangle\right) \\
& +\frac{1}{2 !} \frac{1}{2^{2}} \sum_{j_{1}, k_{1}, j_{2}, k_{2}=1}^{N} r_{j_{1} k_{1}} r_{j_{2} k_{2}}\left(v_{j_{1}} v_{k_{1}} v_{j_{2}} v_{k_{2}}\right. \\
& \left.-\sum^{6}\left\langle v_{j_{1}} v_{k_{1}}\right\rangle v_{j_{2}} v_{k_{2}}+\sum^{3}\left\langle v_{j_{1}} v_{k_{1}}\right\rangle\left\langle v_{j_{2}} v_{k_{2}}\right\rangle\right)+\cdots
\end{aligned}
$$

Then from (A. 6), we have

$$
\begin{aligned}
\langle g\rangle^{\prime} \mid\langle g\rangle= & +\frac{1}{2} \sum_{j, k=1}^{N} r_{j k}\left(\left\langle v_{j} v_{k}\right\rangle^{\prime}-\left\langle v_{j} v_{k}\right\rangle\right) \\
& +\frac{1}{2 !} \frac{1}{2^{2}} \sum_{j_{1}, k_{1}, j_{2}, k_{2}=1}^{N} r_{j_{1} k_{1}} r_{j_{2} k_{2}}\left(\sum^{3}\left\langle v_{j_{1}} v_{k_{1}}\right\rangle^{\prime}\left\langle v_{j_{2}} v_{k_{2}}\right\rangle^{\prime}\right. \\
& \left.-\sum^{6}\left\langle v_{j_{1}} v_{k_{1}}\right\rangle\left\langle v_{j_{2}} v_{k_{2}}\right\rangle^{\prime}+\sum^{3}\left\langle v_{j_{1}} v_{k_{1}}\right\rangle\left\langle v_{j_{2}} v_{k_{2}}\right\rangle\right)+\cdots .
\end{aligned}
$$

Setting $\left\langle\left\langle v_{j} v_{k}\right\rangle\right\rangle=\left\langle v_{j} v_{k}\right\rangle^{\prime}-\left\langle v_{j} v_{k}\right\rangle=\left\langle\left\langle v_{k} v_{j}\right\rangle\right.$, we have

$$
\begin{aligned}
\langle g\rangle^{\prime} /\langle g\rangle=1 & +\sum_{j, k=1}^{N} r_{j k}\left\langle\left\langle v_{j} v_{k}\right\rangle\right\rangle \\
& +\frac{1}{2 !} \frac{1}{2^{2}} \sum_{j_{1}, k_{1}, j_{2}, k_{2}=1}^{N} r_{j_{1} k_{1}} r_{j_{2} k_{2}} \sum^{3}\left\langle\langle v _ { j _ { 1 } } v _ { k _ { 1 } } \rangle \left\langle\left\langle v_{j_{2}} v_{k_{2}}\right\rangle+\cdots\right.\right.
\end{aligned}
$$

If we set $f_{l}=\frac{1}{2 l} \operatorname{trace}\left\{\left(K^{\prime}-K\right) R\right\}^{l}$, a little computation of combinatorics will show that

$$
\begin{aligned}
\langle g\rangle^{\prime} /\langle g\rangle & =\sum_{m=0}^{\infty} \sum_{\mu_{1}+2 \mu_{2}+\cdots+m \mu_{m}=m} \frac{1}{\mu_{1} !} f_{1}^{\mu_{1}} \frac{1}{\mu_{2} !} f_{2}^{\mu_{2}} \ldots \frac{1}{\mu_{m} !} f_{m}^{\mu_{m}} \\
& =\exp \sum_{l=0}^{\infty} f_{l}=\operatorname{det}\left\{1-\left(K^{\prime}-K\right) R\right\}^{-\frac{1}{2}}
\end{aligned}
$$

Combining (A. 20) and (A. 21) with (A. 10), we conclude that the 
definitions of $G(W, t)$ and $\widetilde{G}(W, t)$ does not depend on the choice of expectation values.

There exists a unique $\mathbb{C}[[t]]$-linear anti-isomorphism * $A(W)[[t]] \rightarrow$ $A(W)[[t]]$ characterized by $w^{*}=\sqrt{-1} w$ for $w \in W$.

We denote by $\mathrm{Nr}_{K}$ (resp. $\mathrm{Nr}_{-t_{K}}$ ) the norm with respect to the expectation value $K\left(\right.$ resp. $\left.-{ }^{t} K\right)$. If $\mathrm{Nr}_{K}(g)=\langle g\rangle \exp \frac{1}{2} \sum_{j, k=1}^{N} r_{j k} v_{j} v_{k}$ where $\langle g\rangle \in \mathbb{C}[[t]]$ and $r_{j k}=r_{k j} \in \mathfrak{m}$, we have

$$
\mathrm{Nr}_{-t_{K}} g^{*}=\langle g\rangle \exp \frac{-1}{2} \sum_{j, k=1}^{N} r_{j k} v_{j} v_{k} .
$$

Hence using (A. 20) and (A. 21), we have

$$
\text { (A. 23) } \quad \mathrm{Nr}_{K}\left(g^{*}\right)=\langle g\rangle \operatorname{det}(1+J R)^{-\frac{1}{2}} \exp \frac{1}{2} \sum_{j, k=1}^{N} r_{j k}^{*} v_{j} v_{k} \text {, }
$$

where $R^{*}=-R(1+J R)^{-1}$.

Remark. In the orthogonal case, we have

$$
\left(\text { A. 23)' } \quad \operatorname{Nr}\left(g^{*}\right)=\langle g\rangle \operatorname{det}(1+H R)^{\frac{1}{2}} \exp \frac{1}{2} \sum_{j, k=1}^{N} r_{j k}^{*} v_{j} v_{k}\right.
$$

where $R^{*}=-R(1+H R)^{-1}$.

The product formula for operators in $\widetilde{G}(W, t)$ is also a corollary of transformation formulas (A. 20) and (A. 21).

Let $\Lambda=\left(\lambda_{\mu \nu}\right)_{\nu, \nu=1, \cdots, n}$ be a symmetric matrix with $\lambda_{\nu \nu}=1(\nu=1, \cdots, \mathrm{n})$. Let $W^{(\nu)}(\nu=1, \cdots, n)$ (resp. $\left.v_{j}^{(\nu)}\right)$ be a copy of $W$ (resp. $\left.v_{j}\right)$. We consider the symplectic space $\bigoplus_{\nu=1}^{n} W^{(\nu)}$ equipped with the expectation value $\left\langle v_{j}^{(\mu)} v_{k}^{(\nu)}\right\rangle=\lambda_{\mu \nu}\left\langle v_{j} v_{k}\right\rangle$. Let $g^{(\nu)} \in A\left(W^{(\nu)}\right)[[t]] \subset A\left(\bigoplus_{\lambda=1}^{n} W^{(\lambda)}\right)[[t]] \quad(\nu=1$, $\cdots, n)$ be an operator given by

$$
\operatorname{Nr}\left(g^{(\nu)}\right)=\left\langle g^{(\nu)}\right\rangle \exp \frac{1}{2} \sum_{j, k=1}^{N} r_{j k}^{(\nu)} v_{j}^{(\nu)} v_{k}^{(\nu)},
$$

where $\left\langle g^{(\nu)}\right\rangle \in \mathbb{C}[[t]]$ and $r_{j k}^{(\nu)}=r_{k j}^{(\nu)} \in \mathfrak{m}$. We set

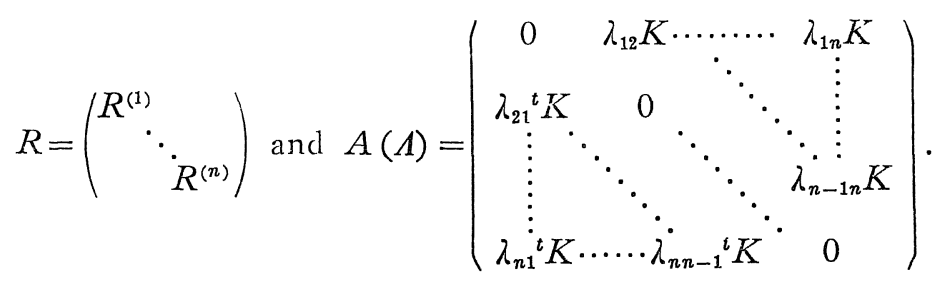


Then we have

$$
\left\langle g^{(1)} \cdots g^{(n)}\right\rangle=\left\langle g^{(1)}\right\rangle \cdots\left\langle g^{(n)}\right\rangle \operatorname{det}(1-A(\Lambda) R)^{-\frac{1}{2}},
$$

$$
\operatorname{Nr}\left(g^{(1)} \cdots g^{(n)}\right)=\left\langle g^{(1)} \cdots g^{(n)}\right\rangle \exp \frac{1}{2} \sum_{\mu, \nu=1}^{n} \sum_{j, k=1}^{N} r_{j k}^{(\mu \nu)} v_{j}^{(\mu)} v_{k}^{(\nu)}
$$

where $\left(\begin{array}{cc}R^{(11)} & \cdots \cdots \cdot R^{(1 n)} \\ \vdots & \vdots \\ R^{(n 1)} & \cdots \cdots \cdot R^{(n n)}\end{array}\right)=R(1-A(\Lambda) R)^{-1}$

Remark. In the above if we set $\lambda_{\mu \nu}=1$ and identify $n$ copies $v_{j}^{(\nu)}$ $(\nu=1, \cdots, n)$ with the original ones, we get formulas in the original space $W$. Since we do not assume the non-degeneracy of the inner product, the specialization to $\lambda_{\mu \nu}=1$ does not violate our arguments. We note also that the analogous results in [1] in the orthogonal case are valid without the assumption of the non-degeneracy of the inner product. (Cf. Remark 1. in p. 256 [1] and Remark in p. 16 [2].)

If $w_{1}, \cdots, w_{k} \in W$, (A. 5) implies that

$$
\left\langle w_{1} \cdots w_{k}\right\rangle= \begin{cases}0 & \text { for } k: \text { odd } \\ \Sigma^{\prime}\left\langle w_{j_{1}} w_{j_{2}}\right\rangle \cdots\left\langle w_{j_{k-1}} w_{j_{k}}\right\rangle & \text { for } k: \text { even }\end{cases}
$$

where $\Sigma^{\prime}$ means the summation over $(k-1)$ !! pairings satisfying $j_{1}<j_{3}$ $<\cdots<j_{k-1}$ and $j_{1}<j_{2}, \cdots, j_{k-1}<j_{k}$. In the orthogonal case, we have

$(\mathrm{A} .26)^{\prime}\left\langle w_{1} \cdots w_{k}\right\rangle$

$$
= \begin{cases}0 & \text { for } k: \text { odd } \\
\sum^{\prime} \operatorname{sgn}\left(\begin{array}{l}
1 \cdots k \\
j_{1} \cdots j_{k}
\end{array}\right)\left\langle w_{j_{1}} w_{j_{2}}\right\rangle \cdots\left\langle w_{j_{k-1}} w_{j_{k}}\right\rangle & \text { for } k: \text { even. }\end{cases}
$$

These formulas are generalized as follows. We explain the symplectic case. We also omit $\Lambda$, since it is nothing more than a special class of expectation values. Let $g_{\nu}(\nu=1, \cdots, n)$ be an operator given by $\operatorname{Nr}\left(g_{\nu}\right)$ $=\left\langle g_{\nu}\right\rangle e^{\rho_{\nu} / 2}$ where $\left\langle g_{\nu}\right\rangle \in \mathbb{C}[[t]]$ and $\rho_{\nu} \in S^{2}(W) \underset{\boldsymbol{C}}{\otimes} \mathrm{m}$. Let $g_{\nu}^{\prime}(\nu=1, \cdots, n)$ be an operator given by $\mathrm{Nr}\left(g_{\nu}^{\prime}\right)=w_{\nu} \mathrm{Nr}\left(g_{\nu}\right)$ where $w_{\nu} \in W \underset{\boldsymbol{C}}{\otimes}[[t]]$. Let $\left\{\nu_{1}, \cdots, \nu_{k}\right\} \quad\left(1 \leqq \nu_{1}<\cdots<\nu_{m} \leqq n\right)$ be a subset of $\{1, \cdots, n\}$. We set

$$
a_{\left\{\nu_{1}, \cdots, \nu_{k}\right\}}=\left\langle h_{1} \cdots h_{n}\right\rangle /\left\langle g_{1} \cdots g_{n}\right\rangle
$$

where 


$$
h_{\nu}=\left\{\begin{array}{lll}
g_{\nu} & \text { if } & \nu \notin\left\{\nu_{1}, \cdots, \nu_{k}\right\} \\
g_{\nu}^{\prime} & \text { if } \quad \nu \in\left\{\nu_{1}, \cdots, \nu_{k}\right\} .
\end{array}\right.
$$

We also denote the elementary quantities $a_{\left\{v_{j}, \nu^{\prime}\right\}}\left(1 \leqq j<j^{\prime} \leqq k\right)$ by $a_{j j^{\prime}}$. Then we have

$$
\text { (A. 27) } \quad a_{\left\{\nu_{1}, \cdots, \nu_{k}\right\}}= \begin{cases}0 & \text { for } k \text { :odd } \\ \sum^{\prime} a_{j_{1} j_{2}} \cdots a_{j_{k-1} j_{k}} & \text { for } k: \text { even }\end{cases}
$$

An analogous formula for the orthogonal case is a corollary to (1.4.10). In this case we need $\operatorname{sgn}\left(\begin{array}{ccc}1 & \cdots & k \\ j_{1} \cdots & j_{k}\end{array}\right)$, and obtain the expression of the form

$$
\begin{cases}0 & \text { for } k: \text { odd } \\
\sum^{\prime} \operatorname{sgu}\left(\begin{array}{l}
1 \cdots k \\
j_{1} \cdots j_{k}
\end{array}\right) a_{j_{1} j_{2}} \cdots a_{j_{k-1} j_{k}} & \text { for } k: \text { even } .\end{cases}
$$

This quantity is called the Pfaffian of the anti-symmetric matrix

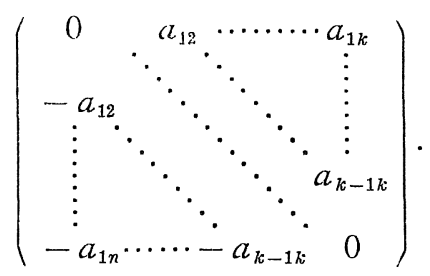

On the other hand the quantity (A.27) is called the Hafnian of the symmetric matrix $\left(\begin{array}{ccc}a_{1} & a_{12} \cdots \cdots \cdots a_{1 k}\end{array}\right)$, where the diagonal elements $\left(\begin{array}{ccccc}a_{1} & a_{12} & \cdots & \cdots & a_{1 k} \\ & \ddots & & \vdots \\ a_{12} & a_{2} & \ddots & \vdots \\ \vdots & \ddots & \ddots & \ddots & \vdots \\ \vdots & \ddots & \ddots & a_{k-1 k} \\ \vdots & \ddots & \ddots & \\ a_{1 k} & \cdots & a_{k-1 k} & a_{k}\end{array}\right)$, where the diagonal elements are arbitrary.

We shall give a simple proof of (A. 2T). The idea of the proof is the same as that of Theorem 1.5.8.

We denote by $W^{*}$ the dual vector space of $W$, and by $\xi_{1}, \cdots, \xi_{N}$ the dual basis of the basis $v_{1}, \cdots, v_{N \text {. }}$ Let $W^{*(\nu)}\left(\operatorname{resp} . \xi_{j}^{(\nu)}\right)(\nu=1, \cdots, n)$ be a copy of $W^{*}\left(\right.$ resp. $\left.\xi_{j}\right)$. We set $\widetilde{W}=W \oplus W^{*(1)} \oplus \cdots \oplus W^{*}(n)$. We extend the expectation value (hence the inner product, too) in $W$ to $\widetilde{W}$ trivially. Namely, we set $\left\langle v_{j} \xi_{k}^{(\nu)}\right\rangle=\left\langle\xi_{k}^{(\nu)} v_{j}\right\rangle=\left\langle\xi_{j}^{(\mu)} \xi_{k}^{(\nu)}\right\rangle=0$. We denote by $W^{(\nu)}$ the dual vector space of $W^{*(\nu)}$ and by $c^{(\nu)}$ the natural isomorphism 
$\ell^{(\nu)}: W \rightarrow W^{(\nu)}$. We define an operator $\widetilde{g}_{\nu} \in A(\widetilde{W})[[t]]$ by $\operatorname{Nr}\left(\widetilde{g}_{\nu}\right)=\left\langle g_{\nu}\right\rangle$ $\times \exp \left(\frac{\rho_{\nu}}{2}+t \sum v_{j} \xi_{j}^{(\nu)}\right)$. Setting $h_{\nu}=\left\{\begin{array}{l}g_{\nu} \text { if } \nu \notin\left\{\nu_{1}, \cdots, \nu_{k}\right\} \\ g_{\nu}^{\prime} \text { if } \nu \in\left\{\nu_{1}, \cdots, \nu_{k}\right\},\end{array}\right.$ we have $\left\langle h_{1} \cdots h_{n}\right\rangle$ $=\left.\left\{\delta\left(\iota^{\left(\nu_{1}\right)}\left(w_{\nu_{1}}\right)\right) \cdots \delta\left(\iota^{\left(\nu_{k}\right)}\left(w_{\nu_{k}}\right)\right) \operatorname{Nr}\left(\widetilde{g}_{1} \cdots \widetilde{g}_{n}\right) / t^{k}\right\}\right|_{v=\xi=0}$. Here $v=\xi=0$ means the natural projection $S(W)[[t]] \rightarrow S^{0}(W) \otimes C[[t]]$. Thus the problem reduces to the one in $S(\widetilde{W})[[t]]$. Notice the formula (A. 25) which states that the product $\widetilde{g}_{1} \cdots \widetilde{g}_{n}$ is of the form $\left\langle\widetilde{g}_{1} \cdots \widetilde{g}_{n}\right\rangle \exp (\tilde{\rho} / 2)$ with $\tilde{\rho} \in S(\widetilde{W})[[t]]$. Hence the following lemma completes our proof. We leave the proof of the lemma to the reader.

Lemma. If $g=\langle g\rangle e^{\rho / 2}$ with $\rho \in S(W)[[t]]$ and $\eta_{\nu} \in W^{*} \quad(\nu=1, \cdots$, $k$ ), we have

$$
\begin{aligned}
& \left.\delta\left(\eta_{1}\right) \cdots \delta\left(\eta_{n}\right) g\right|_{v=0} \\
& =\left\{\begin{array}{lc}
0 & \text { if } \quad k=2 m+1 \\
\langle g\rangle & \left.\left.\sum_{\substack{j_{1}<j_{3}<\cdots, j_{2 m-1} j_{1}<j_{2 m-1}<j_{2 m} \\
j_{1}<j_{2}}} \delta\left(\eta_{j_{1}}\right) \delta\left(\eta_{j_{2}}\right) e^{\rho / 2}\right|_{v=0} \cdots \delta\left(\eta_{j_{2 m-1}}\right) \delta\left(\eta_{j_{2 m}}\right) e^{\rho / 2}\right|_{v=0}
\end{array}\right. \\
& \text { if } \quad k=2 m .
\end{aligned}
$$

The generalization to the case where $\operatorname{Nr}\left(g_{\nu}^{\prime}\right)=w_{\nu, 1} \cdots w_{\nu, k_{\nu}} \operatorname{Nr}\left(g_{\nu}\right)$ is straightforward. In this case we need the quantities of the form

$$
\left\langle g_{1} \cdots: w_{1} w_{2} e^{\rho_{\nu} / 2}: \cdots g_{n}\right\rangle /\left\langle g_{1} \cdots g_{n}\right\rangle
$$

in the Hafnian or the Pfaffian.

The above result asserts that the quantities of the form $\left\langle g_{1} \cdots: w_{1} e^{\rho_{\mu} / 2}\right.$ : $\left.\cdots: w_{2} e^{\rho_{\nu} / 2}: \cdots g_{n}\right\rangle /\left\langle g_{1} \cdots g_{n}\right\rangle$ and $\left\langle g_{1} \cdots: w_{1} w_{2} e^{\rho_{\nu} / 2}: \cdots g_{n}\right\rangle /\left\langle g_{1} \cdots g_{n}\right\rangle$ are elementary. Now we shall give formulas for such quantities. We return to the formulation including $\Lambda$.

We set $\operatorname{Nr}\left(g^{\left(\nu_{l}\right)}\right)=w_{l}^{\prime} \operatorname{Nr}\left(g^{\left(\nu_{l}\right)}\right)$ where $w_{l}^{\prime}=\sum_{\mu=1}^{n} \sum_{j=1}^{N} v_{j}^{(\mu)} c_{l, j}^{\prime(\mu)} \quad(l=1,2)$, with $c_{l, j}^{\prime(\mu)} \in C[[t]]$. Then we have for $1 \leqq \nu_{1}, \nu_{2} \leqq n^{(*)}$

$$
\begin{gathered}
\left\langle g^{(1)} \cdots g^{\left(\nu_{1}-1\right)} g^{\prime\left(\nu_{1}\right)} g^{\left(\nu_{1}+1\right)} \cdots g^{\left(\nu_{2}-1\right)} g^{\prime\left(\nu_{2}\right)} g^{\left(\nu_{2}+1\right)} \cdots g^{(n)}\right\rangle \\
\left\langle g^{(1)} \cdots g^{(n)}\right\rangle \\
=\left({ }^{t} c_{1}^{\prime(1)}, \cdots,{ }^{t} c_{1}^{\prime(n)}\right)\left(\begin{array}{ccc}
\lambda_{11} \widetilde{K} \cdots \lambda_{1 n} \widetilde{K} \\
\vdots & \vdots \\
\lambda_{n 1} \widetilde{K} \cdots \lambda_{n n} \widetilde{K}
\end{array}\right)\left(\begin{array}{c}
c_{2}^{\prime(1)} \\
\vdots \\
c_{2}^{\prime(n)}
\end{array}\right)
\end{gathered}
$$

(*) If $\nu_{1}=\nu_{2}$, we set $\operatorname{Nr}\left(g^{\prime\left(\nu_{1}\right)}\right)=w_{1}^{\prime} w_{2}^{\prime} \operatorname{Nr}\left(g^{\left(\nu_{1}\right)}\right)$. 


$$
\begin{aligned}
& +\left({ }^{t} c_{1}^{\prime(1)}, \cdots,{ }^{t} c_{1}^{\prime(n)}\right)\left(\begin{array}{cccc}
\lambda_{11}{ }^{t} K \cdots \lambda_{1 \nu_{1}-1}{ }^{t} K & 0 & \lambda_{1 \nu_{1}+1} K \cdots \lambda_{1 n} K \\
\vdots & \vdots & \vdots & \vdots \\
\lambda_{n 1}{ }^{t} K \cdots \lambda_{n \nu_{1}-1}{ }^{t} K & 0 & \lambda_{n \nu_{1}+1} K \cdots \lambda_{n n} K
\end{array}\right) \\
& \times R(1-A(\Lambda) R)^{-1}\left(\begin{array}{ccc}
\lambda_{11} K & \cdots & \lambda_{1 n} K \\
\vdots & & \vdots \\
\lambda_{\nu_{2}-11} K & \cdots & \lambda_{\nu_{2}-1 n} K \\
0 & \cdots & 0 \\
\lambda_{\nu_{2}+11}{ }^{t} K & \cdots & \lambda_{\nu_{2}+1 n}{ }^{t} K \\
\vdots & & \vdots \\
\lambda_{n 1}{ }^{t} K & \cdots & \lambda_{n n}{ }^{t} K
\end{array}\right)\left(\begin{array}{c}
c_{2}^{\prime(1)} \\
\vdots \\
\vdots \\
\vdots \\
\vdots \\
c_{2}^{\prime(n)}
\end{array}\right)
\end{aligned}
$$

where ${ }^{t} c_{l}^{\prime(\mu)}=\left(c_{l, 1}^{\prime(\mu)}, \cdots, c_{l, n}^{\prime(\mu)}\right) \quad(l=1,2 ; \mu=1, \cdots, n)$, and

$$
\widetilde{K}=\left\{\begin{array}{lll}
K & \text { if } & \nu_{1}<\nu_{2} \\
0 & \text { if } & \nu_{1}=\nu_{2} \\
{ }^{t} K & \text { if } & \nu_{1}>\nu_{2}
\end{array} .\right.
$$

From (A. 10) and (A. 25) we obtain the formula for $\left\langle w_{1} w_{2} g^{(1)} \cdots g^{(n)}\right\rangle$ where $w_{l}=\sum_{j=1}^{N} \sum_{\nu=1}^{n} v_{j}^{(\nu)} c_{l j}^{(\nu)}(l=1,2), c_{l j}^{(\nu)} \in C[[t]]$. In general, denoting by $c_{l}^{(\nu)}(l=1,2 ; \nu=1, \cdots, n)$ the column vector $\left(\begin{array}{c}c_{l 1}^{(\nu)} \\ \vdots \\ c_{l N}^{(\nu)}\end{array}\right)$, we have for $1 \leqq \nu_{1}$ $\leqq \nu_{2} \leqq n$,

(A. 29) $\frac{\left\langle g^{(1)} \cdots g^{\left(\nu_{1}-1\right)} w_{1} g^{\left(\nu_{1}\right)} \cdots g^{\left(\nu_{2}-1\right)} w_{2} g^{\left(\nu_{2}\right)} \cdots g^{(n)}\right\rangle_{A}}{\left\langle g^{(1)} \cdots g^{(n)}\right\rangle_{A}}$

$$
\begin{aligned}
& =\left({ }^{t} c_{1}^{(1)}, \cdots,{ }^{t} c_{1}^{(n)}\right)\left(\begin{array}{cc}
\lambda_{11} K \cdots \lambda_{1 n} K \\
\vdots & \vdots \\
\lambda_{n 1} K \cdots \lambda_{n n} K
\end{array}\right)\left(\begin{array}{c}
c_{2}^{(1)} \\
\vdots \\
c_{2}^{(n)}
\end{array}\right)
\end{aligned}
$$

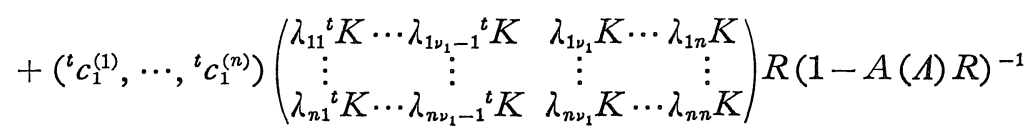

$$
\begin{aligned}
& \times\left(\begin{array}{ccc}
\lambda_{11} K & \cdots & \lambda_{1 n} K \\
\vdots & & \vdots \\
\lambda_{\nu_{2}-11} K & \cdots & \lambda_{\nu_{2}-1 n} K \\
\lambda_{\nu_{2}}{ }^{t} K & \cdots & \lambda_{\nu_{2} n}{ }^{t} K \\
\vdots & & \vdots \\
\lambda_{n 1}{ }^{i} K & \cdots & \lambda_{n n}{ }^{t} K
\end{array}\right)\left(\begin{array}{c}
c_{2}^{(1)} \\
\vdots \\
\vdots \\
\vdots \\
c_{2}^{(n)}
\end{array}\right),
\end{aligned}
$$

(A. 30) $\frac{\left\langle g^{(1)} \cdots g^{\left(\nu_{1}-1\right)} w_{1} g^{\left(\nu_{1}\right)} \cdots g^{\left(\nu_{2}-1\right.} g^{\prime\left(\nu_{2}\right)} g^{\left(\nu_{2}+1\right)} \cdots g^{(n)}\right\rangle_{A}}{\left\langle g^{(1)} \cdots g^{(n)}\right\rangle_{A}}$

$$
=\left({ }^{t} c_{1}^{(1)}, \cdots,{ }^{t} c_{1}^{(n)}\right)\left(\begin{array}{ccc}
\lambda_{11} \widetilde{K} & \cdots & \lambda_{1 n} \widetilde{K} \\
\vdots & & \vdots \\
\lambda_{n 1} \widetilde{K} \cdots \lambda_{n n} \widetilde{K}
\end{array}\right)\left(\begin{array}{c}
c_{2}^{\prime(1)} \\
\vdots \\
c_{2}^{\prime}(n)
\end{array}\right)
$$




$$
\begin{aligned}
& +\left({ }^{t} c_{1}^{(1)}, \cdots,{ }^{t} c_{1}^{(n)}\right)\left(\begin{array}{cccc}
\lambda_{11}{ }^{t} K \cdots \lambda_{1 \nu_{1}-1}{ }^{t} K & \lambda_{1 \nu_{1}} K \cdots & \lambda_{1 n} K \\
\vdots & \vdots & \vdots & \vdots \\
\lambda_{n 1}{ }^{t} K \cdots & \lambda_{n \nu_{1}-1}{ }^{t} K & \lambda_{n \nu_{1}} K \cdots \lambda_{n n} K
\end{array}\right) \\
& \times R(1-A(\Lambda) R)^{-1}\left(\begin{array}{ccc}
\lambda_{11} K & \cdots & \lambda_{1 n} K \\
\vdots & \vdots \\
\lambda_{\nu_{2}-11} K & \cdots & \lambda_{\nu_{2}-1 n} K \\
0 & \cdots & 0 \\
\lambda_{\nu_{2}+11}{ }^{t} K \cdots \lambda_{\nu_{2}+1 n}{ }^{t} K \\
\vdots \\
\lambda_{n 1}{ }^{i} K & \cdots \lambda_{n n}{ }^{t} K
\end{array}\right)\left(\begin{array}{c}
c_{2}^{\prime(1)} \\
\vdots \\
\vdots \\
\vdots \\
\vdots \\
c_{2}^{\prime}{ }^{(n)}
\end{array}\right),
\end{aligned}
$$

where $\quad \widetilde{K}=\left\{\begin{array}{lll}K & \text { if } & \nu_{1} \leqq \nu_{2} \\ { }^{t} K & \text { if } & \nu_{1}>\nu_{2}\end{array}\right.$.

Analogous formulas in the orthogonal case are valid if we replace ${ }^{t} K$ by $-{ }^{t} K$.

If we set $g^{\prime\left(\nu_{2}\right)}=w_{2} g^{\left(\nu_{2}\right)}=: w_{2}^{\prime} \operatorname{Nr}\left(g^{\left(\nu_{2}\right)}\right):$, we have

$$
\left(\begin{array}{c}
c_{2}^{\prime(1)} \\
\vdots \\
\vdots \\
c_{2}^{\prime(n)}
\end{array}\right)=\left(\begin{array}{c}
c_{2}^{(1)} \\
\vdots \\
\vdots \\
c_{2}^{(n)}
\end{array}\right)+\left(\begin{array}{c}
0 \\
\vdots \\
c \\
\vdots \\
0
\end{array}\right) \nu_{2}
$$

where $\quad c=\sum_{\mu=1}^{n} R^{\left(\nu_{2}\right)} \lambda_{\nu_{2} \mu}{ }^{t} K c_{2}^{(\mu)}$. Then (A. 29) and (A. 30) give two different expressions for the same quantity. We shall give a direct proof of the coincidence of these two(*). For the sake of simplicity we assume that $\nu_{1} \leqq \nu_{2}$.

We set

$$
\begin{aligned}
& \Lambda_{1}=\left(\begin{array}{ccc}
\lambda_{11} & \cdots & \lambda_{1 \nu_{1}-1} \\
\vdots & \vdots \\
\lambda_{n 1} & \cdots & \lambda_{n_{1}-1}
\end{array}\right), \quad \Lambda_{2}=\left(\begin{array}{ccc}
\lambda_{1 \nu_{1}} & \cdots & \lambda_{1 n} \\
\vdots & & \vdots \\
\lambda_{n \nu_{1}} & \cdots & \lambda_{n n}
\end{array}\right), \\
& \Lambda_{1}^{\prime}=\left(\begin{array}{ccc}
\lambda_{11} & \cdots & \lambda_{1 n} \\
\vdots & & \vdots \\
\lambda_{\nu_{2}-11} & \cdots & \lambda_{\nu_{2}-1 n}
\end{array}\right), \quad \Lambda_{2}^{\prime}=\left(\begin{array}{ccc}
\lambda_{\nu_{2}+11} & \cdots & \lambda_{\nu_{2}+1 n} \\
\vdots & & \vdots \\
\lambda_{n 1} & \cdots & \lambda_{n n}
\end{array}\right) \\
& { }^{t} c_{1}=\left({ }^{t} c_{1}^{(1)}, \cdots,{ }^{t} c_{1}^{(n)}\right),{ }^{t} c_{2}=\left({ }^{t} c_{2}^{(1)}, \cdots,{ }^{t} c_{2}^{(n)}\right) .
\end{aligned}
$$

Then we have

$$
\text { (A. 29) }={ }^{\imath} c_{1} \cdot \Lambda \otimes K \cdot c_{2}
$$

(*) The case when $g^{\prime\left(\nu_{2}\right)}=g^{\left(\nu_{2}\right)} w_{2}$ is similarly proved 


$$
\begin{aligned}
& +{ }^{t} c_{1} \cdot\left(\Lambda_{1} \otimes{ }^{t} K, \Lambda_{2} \otimes K\right) \cdot R(1-A(\Lambda) R)^{-1} \cdot\left(\begin{array}{c}
\Lambda_{1}^{\prime} \otimes K \\
0 \cdots \cdots 0 \\
\Lambda_{2}^{\prime} \otimes{ }^{t} K
\end{array}\right) \cdot c_{2} \\
& +{ }^{t} c_{1}\left(\Lambda_{1} \otimes{ }^{i} K, \Lambda_{2} \otimes K\right) \cdot R(1-A(\Lambda) R)^{-1} A\left(\begin{array}{c}
0 \\
\vdots \\
c \\
\vdots \\
0
\end{array}\right) \\
& +{ }^{t} c_{1}\left(\Lambda_{1} \otimes{ }^{t} K, \Lambda_{2} \otimes K\right)\left(\begin{array}{c}
0 \\
\vdots \\
c \\
\vdots \\
0
\end{array}\right) \\
& ={ }^{t} c_{1} \cdot \Lambda \otimes K \cdot\left(c_{2}+\left(\begin{array}{c}
0 \\
\vdots \\
c \\
\vdots \\
0
\end{array}\right)\right) \\
& +{ }^{t} c_{1} \cdot\left(\Lambda_{1} \otimes{ }^{t} K, \Lambda_{2} \otimes K\right) \cdot R(1-A(\Lambda) R)^{-1}\left(\begin{array}{c}
\Lambda_{1}^{\prime} \otimes K \\
0 \cdots \cdots \cdot 0 \\
\Lambda_{2}^{\prime} \otimes{ }^{t} K
\end{array}\right)\left(c_{2}+\left(\begin{array}{c}
0 \\
\vdots \\
c \\
\vdots \\
0
\end{array}\right)\right) \\
& =(\text { A. 30). }
\end{aligned}
$$

Finally, using (A. 23)-(A. 25) we have

$$
g g^{*}=g^{*} g=\langle g\rangle^{2} \operatorname{det}(1+K R)^{-1} .
$$

Hence we conclude that $G(W, t)$ is a group.

Along with Proposition A. 1, we have thus proved the following formula:

$$
\text { (A. 32) } \quad \begin{aligned}
& \operatorname{Nr}(g)=\langle g\rangle e^{\rho / 2}, \quad \rho=\sum_{j, k=1}^{N} r_{j k} v_{j} v_{k} \\
& R=(T-1)\left(K-{ }^{t} K T\right)^{-1} \\
& \langle g\rangle^{2}=\operatorname{nr}(g) \operatorname{det}\left(H^{-1} K-H^{-1 t} K \cdot T\right)^{-1}
\end{aligned}
$$

where $\operatorname{nr}(g)=g^{*} g=g g^{*}$.

\section{References}

[1] Sato, M., Miwa, T. and Jimbo. M., Publ. RIMS, Kyoto Univ., 14 (1978), 223-267. 
[2] - ibid., 15 (1979), 201-278.

[3] —, ibid., 15 (1975), 577-629.

[4] - Proc. Japan Acad., 53A (1977), 6-10.

[5] - ibid., 147-152, 153-158, 183-185.

[6] , ibid., 219-224.

[7] , ibid., 54A (1978). 1-5, 36-41.

[8] - ibid., 221-225.

[9] Jimbo, M., ibid., 263-268.

[10] Sato, M., Miwa, T. and Jimbo, M., Field theory of the 2-dimensional Ising model in the scaling limit, RIMS preprint 207 (1976).

[11] Wu, T. T., McCoy, B. M., Tracy, C. A. and Barouch, E., Phys. Rev., B13 (1976), 316-374.

[12] McCoy, B. M., Tracy, C. A. and Wu, T. T., J. Mathematical Phys., 18 (1977), 1058-1092.

[13] — Phys. Rev. Lett., 38 (1977), 793-796.

[14] Bariev, R. Z., Phys. Lett., 55A (1976), 456-458. 